\title{
Characterization of Coxiella burnetii outbreak strains
}

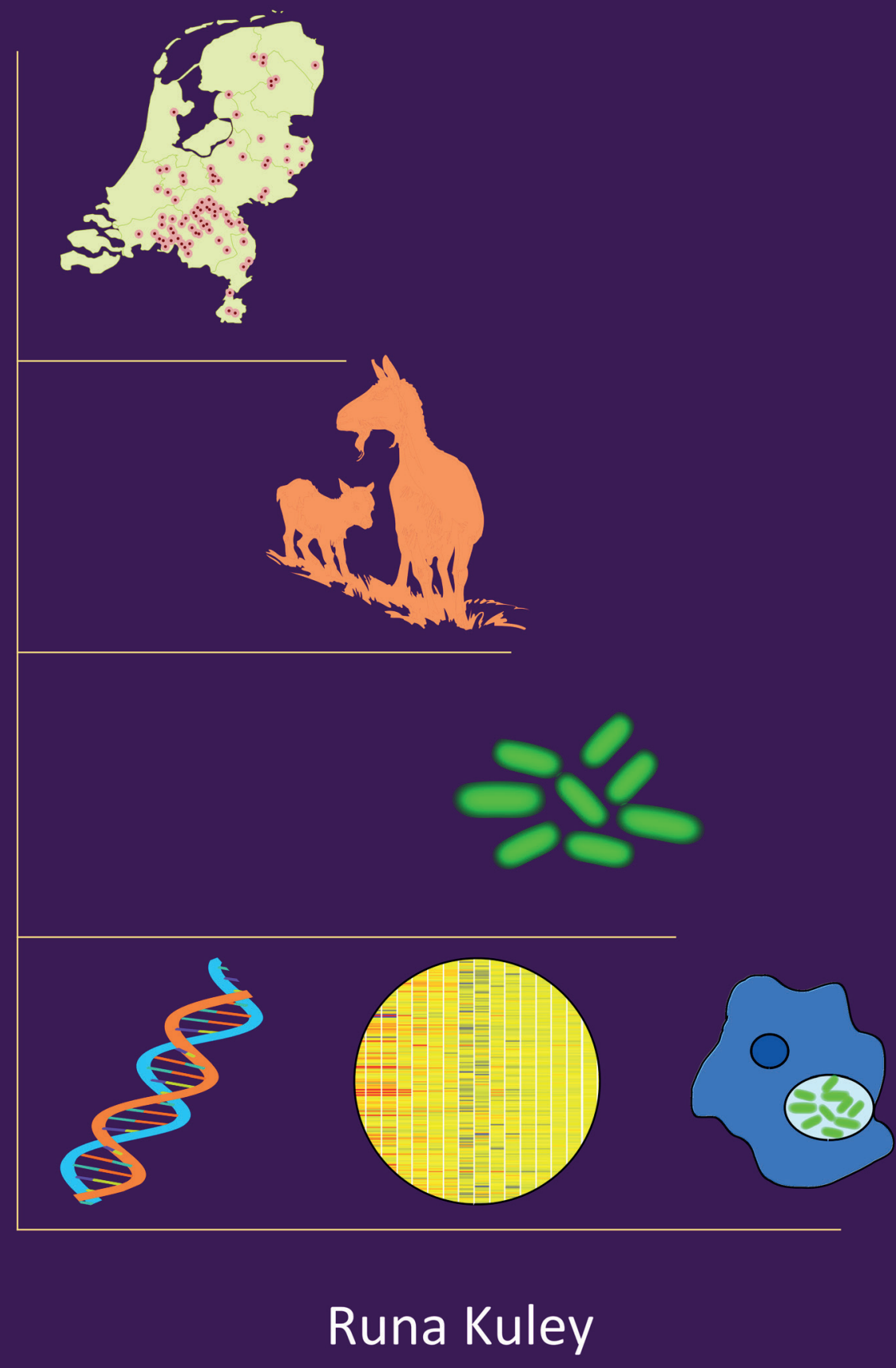





\section{Characterization of Coxiella burnetii outbreak strains}

Runa Kuley 


\section{Thesis committee}

\section{Promotors}

Prof. Dr M.A. Smits

Professor of Intestinal Health of Animals

Wageningen University \& Research

Prof. Dr J.M. Wells

Professor of Host-Microbe Interactomics

Wageningen University \& Research

\section{Co-promotor}

Dr A. Bossers

Senior Scientist, Department of Infection Biology

Wageningen University \& Research

\section{Other members}

Prof. Dr. H.F.J. Savelkoul, Wageningen University \& Research

Prof. Dr J.P.M. van Putten, Utrecht University

Dr A.L. Zomer, Utrecht University

Dr C.A.W. Klaassen, Erasmus MC Rotterdam

This research was conducted under the auspices of the Graduate School of Wageningen Institute of Animal Sciences (WIAS). 


\title{
Characterization of Coxiella burnetii outbreak strains
}

\author{
Runa Kuley
}

\section{Thesis}

submitted in fulfilment of the requirements for the degree of doctor

at Wageningen University

by the authority of the Rector Magnificus

Prof. Dr A.P.J. Mol,

in the presence of the

Thesis Committee appointed by the Academic Board

to be defended in public

on Monday May 8, 2017

at 4 p.m. in the Aula. 


\section{Runa Kuley}

Characterization of Coxiella burnetii outbreak strains, 226 pages

$\mathrm{PhD}$ thesis, Wageningen University, Wageningen, NL (2017)

With references, with summary in English and Dutch

ISBN 978-94-6343-151-4

DOI $10.18174 / 410829$ 




\section{Contents}

Chapter 1: General introduction and thesis outline 9

Chapter 2: Cell-free propagation of Coxiella burnetii does not affect its relative virulence. $\quad 37$

Chapter 3: Major differential gene regulation in Coxiella burnetii between in vivo and in 59 vitro cultivation models

Chapter 4: First complete genome sequence of the Dutch veterinary Coxiella burnetii 85 strain NL3262, originating from the largest global Q fever outbreak, and draft genome sequence of its epidemiologically linked chronic human isolate NLhu3345937

Chapter 5: Plasticity and polymorphisms in critical genes correlate with increased 93 virulence of Dutch outbreak-related Coxiella burnetii strains

Chapter 6: Coxiella burnetii strain specific immune responses are more depending on host origin than on MLVA genotype classification.

Chapter 7: Coxiella burnetii isolates originating from infected cattle induce a more pronounced pro-inflammatory cytokine response compared to isolates from infected goats and sheep.

Chapter 8: General discussion

Summary

Samenvatting

Curriculum vitae

Acknowledgements

Training and supervision plan

Colophon 



\title{
Chapter 1
}

\section{General introduction and thesis}

\author{
outline
}




\section{General Introduction}

Coxiella burnetii is the etiological agent of Q fever; a worldwide zoonotic disease that can result in large outbreaks in humans. In the recent years, there has been an immense improvement in our understanding of both the biology and pathogenicity of $C$. burnetii. However very little is known about the molecular mechanisms involved in its interaction with hosts. In addition, only a few virulence factors have been identified and biologically validated. Virulence factors of this pathogen most likely include determinants promoting its survival and replication in hostile intracellular environments and facilitating manipulation or evasion of host immune responses. Hence, the molecular characterization of C. burnetii isolates is essential for virulence factor analysis, epidemiological knowledge, outbreak investigations, development of diagnostic tools, therapeutic interventions and most importantly for vaccine development. In this chapter, we present an overview of the current knowledge on C. burnetii biology as well as the approaches used to investigate this pathogen and identify virulence factors, which is crucial for development of an effective and safe vaccine for long-term control of $\mathrm{Q}$ fever.

\section{Discovery of the $Q$ fever agent}

Q fever stands for "Query fever" as very little was known about the causative agent and the disease when it was first discovered in the 1930s. Q fever was first described in abattoir workers in Brisbane, Queensland, Australia by Edward Holbrook Derrick [1,2]. Simultaneously, the pathogen was isolated by Frank Macfarlane Burnet and Mavis Freeman from samples sent by Derrick, who hypothesized Q fever was of rickettsial origin [3]. Around the same time Herald Cox and Gordon Davis at the Rocky Mountain Laboratory in Hamilton, independently isolated a new infectious agent from ticks collected at Nine Mile Creek, Montana with rickettsia-like properties [4]. A laboratory worker who became accidently infected by this new agent displayed symptoms remarkably similar to the Q fever agent isolated from abattoir workers in Australia, suggesting a common infectious agent. Further crossprotection studies in mice confirmed these two newly discovered infectious agents to be the same pathogen [5]. The pathogen was initially designated as Rickettsia burneti, on account of its rickettsialike properties [2]. Based on phenotypic characters, the pathogen was later classified under a new genus comprising of only one species, "Coxiella burnetii" in honor of the contribution made by Cox and Burnet.

C. burnetii is highly infectious with estimated $\mathrm{ID}_{50}$ (number of bacilli required to infect $50 \%$ of individuals) of one. This is the lowest $\mathrm{ID}_{50}$ known so far, making it one of the most infectious organisms. Furthermore, the bacteria are highly resistant to heat and desiccation, ubiquitous, dormant in environment for long periods and infectious over several kilometers when aerosolized. Due to these characteristics, C. burnetii could potentially be used as biological weapon and is therefore classified as a category B bioterrorism agent by the Centers for Disease Control (USA) $[6,7]$. 


\section{$Q$ fever disease}

C. burnetii infects a broad range of hosts, but infections are wide-spread in domestic ruminants $[8,9]$. Among these, goats, sheep and cattle are the primary reservoirs of the pathogen and represent the most frequent source of human infection [10]. Infections in animals are assumed to occur mostly by inhalation of the pathogen from the environment and are often asymptomatic [11]. The disease symptoms are usually manifested in pregnant animals and widely differ between hosts $[8,10,12]$. In pregnant goats and sheep, the main clinical manifestations are abortions at final gestation stage. Infected cattle usually do not show symptoms although abortions, subfertility and metritis are seen in some cases [13]. Abortions in infected animals result in excretion of large numbers of C. burnetii $\left(10^{9} /\right.$ gram placenta) into the environment. Inhalation of contaminated aerosols from the environment are the main risk factor for infection in humans [8,9,13,14]. Following infection, $60 \%$ of infected humans are usually asymptomatic. In symptomatic patients, the clinical signs of acute infections vary greatly but generally manifest as flu-like illness and pneumonia. Following acute Q fever, a persistent fatigue condition causing significant disabilities in the daily life of the individual is referred to as $\mathrm{Q}$ fever fatigue syndrome (QFS). QFS has been described and well documented in many countries following an outbreak, but the cause of development of this condition and proper treatment course are still unknown $[15,16]$. Around $1-5 \%$ of the clinical cases can result in chronic infections after months or years of contracting the disease. This occurs mainly in immune deficient individuals or in individuals with pre-existing cardiac valvular disease and often leads to life-threatening endocarditis $[8,13,17-19]$.

\section{$Q$ fever outbreaks in the Netherlands and source identification}

Q fever outbreaks are reported frequently worldwide and have a major public health impact. The outbreak in the Netherlands during 2007-2010 increased the attention to Q fever as more than 4000 human cases were registered. It is by far the largest Q fever outbreak reported in the literature $[17,19,20]$. The factors leading to the outbreak are not fully understood but have been connected with an increase in density of dairy goat farms in the affected area. Other speculated reasons for the outbreak are based on pathogen related factors, including the hyper-virulence of the circulating strains which might have resulted in an increased zoonotic potential [20-25].

Epidemiological studies on the outbreak showed that the increase in human cases of Q fever coincided with an increase in abortions among goats [20,26,27]. Laboratory confirmations of the observed link were conducted by genotyping studies on $C$. burnetii isolates from a large set of samples from dairy goats, sheep, cattle and humans [34]. The "multilocus variable number tandem repeats" (MLVA) [28,30,31] and the "multispacer sequence typing" (MST) [29] methods were used for genotyping to determine strain diversity. A total of 13 MLVA genotypes (CbNL01-CbNL13) of $C$. burnetii were identified in clinical samples. Among these, the CbNL01 genotype was predominantly present in isolates from goats and human patients. Similar results were obtained by MST genotyping, 
showing MST33 genotypes in goat and human isolates. A less prevalent CbNL12 genotype was majorly found to be associated with cattle and to a lesser extent with goat and sheep isolates $[28,29,32,33]$. This genotype was not found among human isolates, implicating the predominant goat associated CbNL01 genotype as the primary source of the human Q fever outbreak in the Netherlands and confirming the speculations of earlier epidemiological studies (Table 1) [20,23,24,28,34].

Table 1. Overview of MLVA genotypes of C. burnetii isolates collected from various hosts and clinical samples during the Q fever outbreak period in the Netherlands [28]

\begin{tabular}{|c|c|c|c|c|c|c|c|c|c|c|c|c|c|c|}
\hline \multirow{2}{*}{ Origin } & \multirow{2}{*}{ Sample type } & \multicolumn{12}{|c|}{ MLVA marker and number of repeats } & \multirow{2}{*}{ Genotype } \\
\hline & & 1 & 3 & 20 & 21 & 22 & 24 & 27 & 28 & 30 & 31 & 34 & 36 & \\
\hline Human & heart valve & 4 & 7 & 19 & 6 & 6 & 11 & 3 & 3 & 5 & 3 & 7 & 13 & CbNL01 \\
\hline Goat & $\begin{array}{l}\text { placenta, vaginal swab, fetal } \\
\text { tissue, bulk tank milk }\end{array}$ & 4 & 7 & 19 & 6 & 6 & 11 & 3 & 3 & 5 & 3 & 7 & 13 & CbNL01 \\
\hline Sheep & placenta & - & 7 & - & 6 & 6 & 11 & 3 & 3 & 5 & 3 & 7 & 13 & CbNL01 \\
\hline Goat & placenta & 3 & 6 & 15 & 6 & 6 & 13 & 2 & 7 & 6 & 3 & 9 & 4 & CbNL12 \\
\hline Sheep & placenta & - & 6 & - & 6 & 6 & 13 & 2 & 7 & 6 & 3 & 9 & 4 & CbNL12 \\
\hline Cattle & placenta & 3 & 6 & 15 & 6 & 6 & 13 & 2 & 7 & 6 & 3 & 9 & 4 & CbNL12 \\
\hline Goat & vaginal swab & - & 7 & - & 6 & 6 & 11 & 3 & 3 & 5 & 3 & 7 & 7 & CbNL02 \\
\hline Goat & vaginal swab & - & 7 & - & 6 & 6 & 11 & - & 3 & 5 & - & 7 & 9 & CbNL03 \\
\hline Goat & vaginal swab & - & 7 & - & 6 & 6 & 11 & 3 & - & 0 & - & 7 & 13 & CbNL04 \\
\hline Goat & vaginal swab & - & 7 & - & 6 & - & - & 3 & 3 & 6 & 3 & 7 & 13 & CbNL05 \\
\hline Goat & vaginal swab & - & 7 & - & 6 & 6 & 11 & 3 & - & 4 & 3 & 7 & - & CbNL06 \\
\hline Goat & vaginal swab & - & 7 & - & 6 & - & - & 0 & 3 & 5 & 3 & 7 & 13 & CbNL07 \\
\hline Goat & vaginal swab & - & 7 & - & 6 & 6 & - & 0 & 0 & 5 & 3 & 7 & 13 & CbNL08 \\
\hline Goat & fetal tissue & - & 7 & - & 6 & - & 11 & 3 & 4 & 5 & 3 & 7 & 2 & CbNL09 \\
\hline Sheep & vaginal swab, bulk tank milk & - & 7 & - & 6 & 6 & 11 & 2 & 3 & 5 & 3 & 3 & - & CbNL10 \\
\hline Goat & fetal tissue & - & 7 & - & 6 & - & 11 & 2 & 0 & 5 & 3 & 7 & 13 & CbNL11 \\
\hline Cattle & placenta & - & 6 & - & 6 & 6 & 13 & 2 & 7 & 6 & 3 & 10 & 4 & CbNL13 \\
\hline
\end{tabular}

Among all, the CbNL01 genotype occurred most predominant followed by CbNL12 genotype

Following the outbreak drastic measures were taken to reduce the spread of the pathogen and control the disease. The measures included large-scale culling of Q fever pregnant goats, mandatory vaccinations, stringent hygiene protocols, bulk tank milk monitoring etc. This resulted in a stabilization of the number of human cases by 2010 . However the number of chronic cases due to outbreak infections is still significant $[20,24,35]$. Hence, Q fever is a significant public health threat that can only be diminished by development of improved diagnostic tools, better therapeutics and effective vaccines. Active research on the $\mathrm{Q}$ fever pathogen and the disease is an absolute requirement as little knowledge on diagnosis and treatment is presently available. 


\section{Life inside the host cell}

C burnetii is an obligate intracellular, small gram-negative bacterium $(0.2$ to $0.4 \mu \mathrm{m}$ wide, 0.4 to $1 \mu \mathrm{m}$ long). Upon infection, C. burnetii enters host cells by phagocytosis and is able to survive within an acidic parasitophorous vacuole (PV) created by fusion of phagosomes and lysosomes (Fig 3) [8,36]. Several intracellular bacteria such as Mycobacteria tuberculosis [37], Chlamydia psittaci [38], Legionella pneumophila [39], Shigellae [40] and Listeria monocytogenes [41] subvert host mechanisms to prevent phagolysosomal fusion to survive in host cells in order to avoid the low $\mathrm{pH}$, oxygen radicals, and degradative proteases of the phagolysosome. Unlike these intracellular pathogens, C. burnetii does not employ any mechanisms to avoid the phagolysosome fusion and requires such harsh acidic conditions to activate its metabolism and successful replication.

C. burnetii has a biphasic life cycle, consisting of small-cell variant (SCV) and a large-cell variant (LCV) morphotypes. The variants are easily distinguishable by electron microscopy and correspond to different developmental stages of C. burnetii [42]. SCVs are metabolically inactive and highly resistant to environmental stress such as osmotic pressure, heat and chemicals. Due to these features, SCVs can survive in the environment for long periods of time. SCV can infect eukaryotic hosts and transform into LCV under acidic conditions of the PV [43]. LCVs are the metabolically active and replicating forms of the bacteria, which can differentiate into spore-like SCVs to complete the developmental cycle [42].

\section{Culturing of $\boldsymbol{C}$. burnetii under laboratory conditions}

During the Q fever outbreak period in the Netherlands, several isolates from different host species were obtained [28] and primarily cultured using the BGM (buffalo green monkey) cell line [11,44]. Cell-based culture is the gold standard method for in vitro growth of $C$. burnetii. The bacteria can grow in a number of cell types such as macrophage-like cell lines $[45,46]$, fibroblast cells $[43,47]$ and Vero cell lines [48]. Apart from cell cultures, C. burnetii also propagates within yolk-sac of embryonated eggs which is a widely used method for culturing large quantities of the bacterium $[33,49]$.

The obligate intracellular nature of $C$. burnetii has led to experimental constraints hindering the research towards a better understanding of the pathogen's disease mechanisms. A recent, significant advance in $\mathrm{Q}$ fever research has been the development of a cell-free growth media for $C$. burnetii. This complex medium known as acidified citrate cysteine medium-2 (ACCM-2) was based on the metabolic requirements of $C$. brunetti and supports axenic growth of $C$. burnetii in liquid broth and on agar plates under microaerophilic conditions. The cultured bacteria are infective and exhibit the characteristic SCV and LCV developmental forms similar to cell-based or in vivo grown organisms [50-52]. Based on ACCM-2, a commercially available defined medium ACCM-D was recently developed for host-cell free culture of C. burnetii [53]. 


\section{C. burnetii genome sequences}

The first complete C. burnetii genome sequence of the Nine Mile I RSA493 (NM) isolate was published in 2003. The genome appeared to be circular and was composed of a 1,995,275 bp chromosome and a 37,393 bp QpH1 plasmid [54]. All C. burnetii isolates sequenced so far carry an autonomous replicating plasmid or the plasmid sequences are integrated into their chromosome. Currently four plasmid types, designated as QpH1 (37 kb), QpRS (39 kb), QpDG (42 kb) and QpDV (33 kb) have been identified. A $16 \mathrm{~kb}$ region is conserved in all plasmids including in the plasmid sequences integrated in the chromosome, implying critical function of this region in bacterial survival [55-57]. Bioinformatics predictions of gene functions in the NM genome showed transportation, biosynthesis and metabolic capabilities, along with a high proportion (33.7\%) of hypothetical genes with unknown function. A unique feature of $C$. burnetii genome sequences is the presence of large number of transposons compared to other obligate intracellular pathogens [58-60]. These transposase genes (insertion sequence elements) are associated with chromosomal rearrangements indicating a high plasticity of the genome (Figure 1) [54]. C. burnetii genomes also harbor pseudogenes suggesting

A

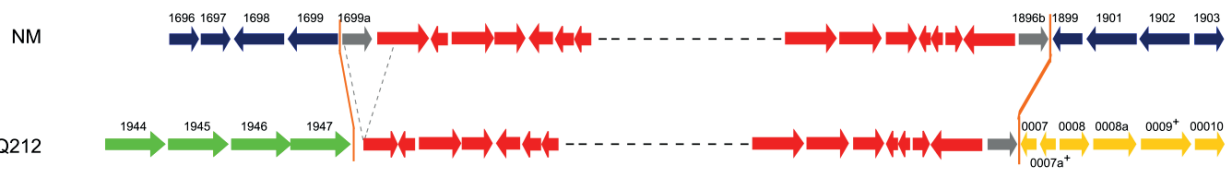

B

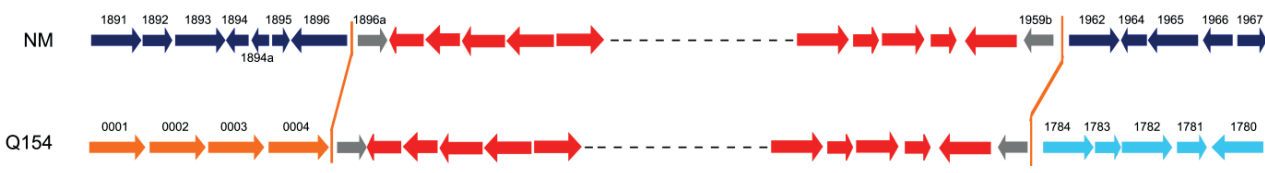

Figure 1. Chromosomal rearrangements due to transposons. Rearrangement of an NM ORF cluster bound by transposase genes (grey ORFs) is shown A) CBU1699a and CBU1896b relative to CbuG_Q212 (Q212) B) CBU1896b and CBU1959b relative to CbuK_Q154 (Q154). The red ORFs and black dotted lines represent ORFs between transposase genes, orange line show ORF cluster boundaries, dotted grey line indicate deletion relative to NM. ORFs in CbuG_Q212 (green and yellow) and CbuK_Q154 (orange and blue) are numbered using orthologs of NM. ORFs denoted with + are frameshifted relative to NM.

an ongoing genomic reduction [66]. The presence of pseudogenes is predicted to be specific genome manifestations of an intracellular lifestyle most probably contributing to bacterial evolution, pathogenicity and host/niche specificity [61,62]. Pseudogenes are also reported in other highly pathogenic bacteria such as Mycobacterium, Leprosy bacillus, Shigella spp and E. coli $[63,58,64-67]$. Thus, disparate collection of pseudogenes also in C. burnetii isolates suggest that these isolates are at different stages of pathoadaptation $[62,68,69]$. 
Apart from the NM genome sequence [54], several other complete and draft genomes sequences are published and available in Genbank (Table 2). The availability of genome sequences from several strains obtained from various hosts and with different clinical phenotypes will enable improved phylogenetic analysis and extended comparative genomic analysis. These approaches may enrich our understanding on strain evolutions and provide information on similarities and differences in genome architecture and gene content between isolates and its effect on virulence and host tropism [71,79]. Previous comparative genomics studies on C. burnetii strains allowed the identification of novel genes, point mutations, large deletions and genome rearrangements shedding light on strain diversity $[62,70,71,78]$. So far, the genetic differences among C. burnetii Dutch outbreak strains are only based on MLVA/MST loci. Information on genetic variations encoded by these strains, which

Table 2. Features of $C$. burnetii genome sequences available in the NCBI database

\begin{tabular}{|c|c|c|c|c|c|c|}
\hline Strains & Accession No. & Genome & GC & Plasmid & CDS & Reference \\
\hline NMRSA493 $^{\mathrm{C}}$ & NC_002971.3 & 1995281 & 42.7 & QpH1 & 2134 & {$[54,62]$} \\
\hline CbRSA331 ${ }^{\mathrm{c}}$ & NC_010117.1 & 2016427 & 42.8 & QpH1 & 1989 & - \\
\hline Dugway $^{c}$ & NC_009727.1 & 2158758 & 42.4 & QpDG & 2060 & [62] \\
\hline CbuG_Q212 ${ }^{\mathrm{C}}$ & NC_011527.1 & 2008870 & 42.6 & integrated & 1853 & [62] \\
\hline CbuK_Q154 & NC_011528.1 & 2063100 & 42.7 & QpRS & 2001 & [62] \\
\hline $\mathrm{Z} 3055^{\mathrm{c}}$ & PRJEB1438 & 1995463 & 42.6 & QpH1 & 1913 & {$[70]$} \\
\hline $\mathrm{Cb} 175^{\mathrm{c}}$ & HG825990.3 & 1989565 & 42.6 & QpH1 & 1363 & [71] \\
\hline $\mathrm{Cb} 109^{\mathrm{d}}$ & AKYP00000000 & 2030000 & 42.5 & QpH1 & 1846 & [72] \\
\hline Q321 ${ }^{\mathrm{d}}$ & AAYJ01000000 & 2004584 & 42.7 & QpDV & 1897 & [73] \\
\hline Goat Q177 ${ }^{\mathrm{d}}$ & NZ_AAUP00000000.2 & 2090565 & 42.6 & QpRS & 1995 & - \\
\hline $\mathrm{Cb}{ }_{-} 2^{\mathrm{d}}$ & CCAJ01000000 & 2013749 & 42.5 & QpH1 & 1949 & {$[30,74]$} \\
\hline $\mathrm{Cb} \_\mathrm{B}^{\mathrm{d}}$ & CCAH01000000 & 2008014 & 42.5 & QpH1 & 1919 & {$[30,74]$} \\
\hline EV-Cb_BK $10^{\mathrm{d}}$ & CCAL01000000 & 1999727 & 42 & unknown & 1909 & [74] \\
\hline Cb_O184 ${ }^{\mathrm{d}}$ & CCAK01000000 & 2168222 & 42.4 & QpRS & 2014 & {$[30,74]$} \\
\hline $\mathrm{EV}-\mathrm{Cb}{ }_{-} \mathrm{C} 13^{\mathrm{d}}$ & CCAM01000000 & 2023172 & 42.6 & unknown & 1939 & [74] \\
\hline Cb_B18 & CCAI01000000 & 2008445 & 42.5 & unknown & 1917 & [74] \\
\hline $\mathrm{Cb} 171^{\mathrm{d}}$ & CDBG00000000.1 & 2077120 & 42.5 & unknown & - & - \\
\hline Dog Utad ${ }^{d}$ & PRJEB4294 & 2008938 & 44.5 & integrated & 1896 & [75] \\
\hline Cb196 & CCXO00000000.1 & 2006415 & 42.9 & QpRS & 1898 & [69] \\
\hline Namibia $^{\mathrm{d}}$ & СР007555 & 2101438 & 41.1 & QpRS & 1979 & [76] \\
\hline $\mathrm{Cb} 185^{\mathrm{d}}$ & NZ_CBTH000000000.1 & 1991515 & 42.6 & unknown & 1806 & - \\
\hline $\mathrm{AuQ01}{ }^{\mathrm{d}}$ & JPVV00000000 & 2073000 & 42.5 & QpRS & 1984 & [77] \\
\hline NL-Limburg ${ }^{\mathrm{d}}$ & JZWL00000000 & 2214254 & 42.8 & QpH1 & 2097 & [78] \\
\hline
\end{tabular}

c: complete genomes (Genome in bp)

d: draft genomes (Genome in bp)

Integrated: plasmid sequences integrated in chromosomes

Unknown: plasmid type not known for these C. burnetii strains 
could have resulted in the epidemic, is still missing. In Chapter 4 and 5 of this thesis we have sequenced several Dutch strains and performed a comparative genome analysis to provide an overview of clonal diversity of these strains in relation to virulence, and deciphered mechanisms adapted by the outbreak strains.

\section{Bacterial virulence}

The intracellular lifestyle of $C$. burnetii has hampered the identification of virulence factors and as a result lipopolysaccharide (LPS) is the major genetically confirmed virulence factor known [80]. Bioinformatics predictions of gene functions based on available genome sequences, has not resulted in the identification of potential C. burnetti (cyto) toxins. Nevertheless, gene functions were identified which are involved in subverting oxidative stress, DNA repair and effector proteins with eukaryoticlike domains which could be involved in modulating host responses. Researchers now consider these factors crucial for pathogenicity and intracellular survival $[54,62,81]$. The $C$ burnetii genome also encompasses several genes encoding hypothetical proteins with unknown functions, a proportion of which are highly conserved across C. burnetii strains [62,70]. Several of these hypothetical proteins are membrane-associated implying their potential involvement in the interaction with the host, whereas other hypothetical proteins could be important for the unique lifestyle of $C$. burnetii. Therefore, functional annotation of the genes with unknown functions will increase our knowledge on pathogenicity and may result in the identification of novel virulence factors.

Phase variation: Presence or absence of LPS largely correlates with "phase variation" in C. burnetii. Phase I bacteria containing an intact LPS are virulent and able to replicate and persist in immunocompetent hosts most likely by avoiding host defense mechanisms. In contrast, phase II

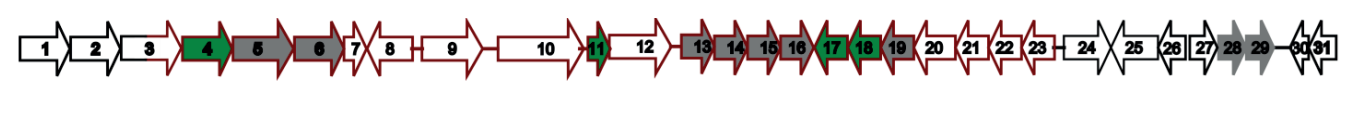

$38 \mathrm{~kb}$

Figure 2. C. burnetii O-antigen part $(38 \mathrm{~kb})$ of LPS encoding genes. The cluster of O-antigen genes, CBU0676CBU0706 is labelled as 1-31. The genes indicated in grey are hypothesized to be involved in O-antigen synthesis and export, whereas the genes indicated in green are hypothesized to be involved in other LPS synthesis steps and carbohydrate metabolism. Genes bordered in red are deleted in the NM phase II strain.

bacteria are mostly characterized by a truncated LPS and are avirulent, being effectively cleared by host immune responses [82]. Phase 1 LPS is also identified as a key determinant of protective immunity [83] as vaccines prepared from phase I bacteria provide better protection against Q fever in human and animals compared to phase II-based vaccines $[8,84,85]$. A shift from phase I to phase II 
forms occurs due to repeated passages of bacteria in in vitro cultures and is often associated with deletion of clusters of genes involved in production of O-antigen part of LPS (Figure 2) [73,86].

Ability to subvert oxidative stress: Within the environment of the PV, C. burnetii is exposed to varying levels of reactive oxygen and nitrogen species (ROS and RNS), which represent the primary defense mechanism of the host cells against invading bacteria. To survive and replicate within this harsh environment, $C$. burnetii encodes numerous enzymes such as superoxide dismutase, catalase and peroxiredoxins (encoded by $\operatorname{sodBC}$, katE, ahpC1C2D) capable of detoxifying deleterious reactive molecules $[62,81]$. The bacteria also encode for a limited numbers of DNA repair genes involved in base excision repair, nucleotide excision repair and recombinational DNA repair (encoded by addAB complex) $[81,87,88]$. Due to presence of limited DNA repair genes, a constitutive expression of these genes might be necessary to maintain the integrity of genome during replication of $C$. burnetii inside host PVs (Figure 3) [81,88].

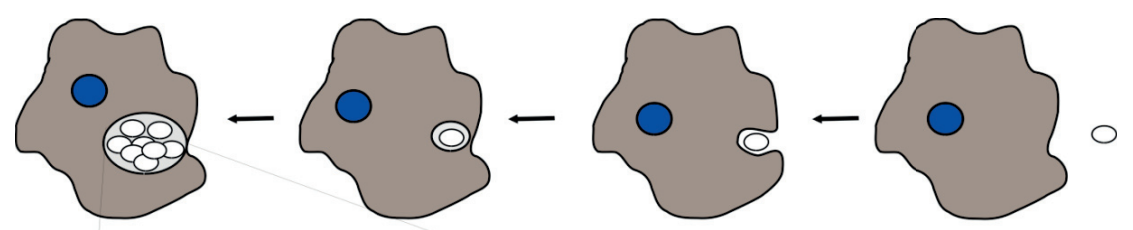

Figure 3. Uptake of C. burnetii by host cells and virulence mechanisms against oxidative stress and intracellular host signaling. C. burnetii enters the host cell by phagocytosis and multiplies in acidic PV. C. burnetii uses oxidative enzymes and DNA repair as defense mechanisms against reactive oxygen species (ROS) and nitrogen species generated by host cells. Manipulation of host responses by $C$. burnetii could be effected by numerous effector proteins, delivered to the host cytosol by the T4SS and other secretion systems. (Acp- acid phosphatase enzyme; Anks- Ankyrin repeat containing proteins). 
Host component modulations: Like other intracellular pathogens, C. burnetii possibly adapts processes resulting in manipulation of host signaling pathways during intracellular growth to ensure a viable, sustainable niche to successfully replicate [89]. C. burnetii encodes several signal sequence-containing enzymes which could actively manipulate intracellular host signaling [90,91]. For example, an acid phosphatase is predicted to inhibit phosphorylation of host proteins and prevent oxidative burst after phagocytosis, helping the bacteria to cope with oxidative stress [62,92]. Additionally, enzymes such as phospholipases and inositol-phosphate phosphatases are speculated to act on host cell lipids and manipulate lipid signaling pathways $[62,90,93]$. Furthermore, C. burnetii encodes multiple eukaryotic like proteins which could mimic activity of host cell proteins [94]. Among these are proteins with the ankyrin repeat regions that interferes with host cell apoptosis pathway [95]. Other examples include two eukaryotic like sterol reductases. These enzymes are predicted to be involved in final stages of cholesterol or novel sterol synthesis using intermediates of host cell lipids, which can have structural or signaling roles $[90,96]$. Other proteins that contain eukaryotic like domains (F boxes, serine/threonine protein kinases, tetratricopeptide repeats, leucine-rich repeats and coiled core domains) are assumed to be interfering with host ubiquitin signaling pathways and in host cell signal transduction [62,97-99]. Many of the effector proteins that are intimately involved in virulence and pathogenesis of $C$. burnetii, are anticipated to translocate into the host cytosol by a Type IV secretion system (T4SS) [54,100,101]. The T4SS is conserved in C. burnetii strains implying the critical function of this protein secretion system in bacterial survival and intracellular replication (Figure 3 ).

\section{Animal models, host specificity and immune responses to $C$. burnetii}

Domestic ruminants such as goat and sheep are the most important source of infection in humans, while a minority of the human infections are attributed to cattle $[8,28,102]$. Strikingly, different disease phenotypes are seen in different $C$. burnetii hosts. Severe forms of disease outcomes, such as abortions, commonly occur in goats, whereas infected cattle usually do not show any clinical symptoms $[8,13,28]$. Epidemiological studies based on seroprevalence, reveal the presence of high percentage of anti-C. burnetii antibodies in cattle herds than in goats and sheep herds in The Netherlands and few other European countries. On the contrary, Q fever outbreaks are rarely linked to cattle [102,103]. Additionally, we also see that only a specific genotype of C. burnetii strains (CbNL12) are associated in with cattle populations in European countries [28,32,102]. Reasons underlying these differences in disease phenotypes, host susceptibility and seroprevalence are not well understood and can be assessed in appropriate animal models.

Mice and guinea pigs are highly susceptible to C. burnetii and are commonly used animal models to study disease pathogenesis, strain virulence and host immune responses [33,80,104-109]. $C$. burnetii infection in immunocompetent guinea pigs and mice by intraperitoneal routes result in systemic infections characterized by hepato-splenomegaly at early infection periods (7-14 days) [110]. The degree of splenomegaly and bacterial load in spleens after infection are regarded important 
parameters pointing towards the virulence potential of $C$. burnetii strains $[33,105,108,109]$. At later infection period (21-28 days) a decrease in the spleen weight and bacterial load is seen implying a clearance of the infection [33,108]. Also in pregnant mice, a systemic infection occurs with an abundant presence of $C$. burnetii in both fetal as well as maternal parts of the placenta, which results in high frequency of abortions and still-births [111]. Although mice models are routinely used, experiments with natural Q fever host model like goats can provide significant insights in accurate diagnosis of infected animals, mechanisms of bacterial pathogenicity, transmission routes and immune responses $[11,14,44,112]$. In contrast to mice model, C. burnetii infections in pregnant goats results in localized infection that are restricted to the trophoblasts of the fetal part of the placenta. As a result, abortions can be the major clinical symptoms in goats after a C. burnetii infection. After abortion or parturition, infected goats are able to gradually clear the infection [11,112].

So far, studies in mice showed the importance of both humoral and cell mediated immune responses for successful host defense against C. burnetii infection [113]. With respect to humoral immunity, IgM and IgG antibodies can be detected in infected mice against $C$. burnetii phase I and II antigens $[33,114]$. The role of cellular mediated immunity in the host defense against C. burnetii infections is not well established. In general, macrophages and other mononuclear cells are primary target cells of $C$. burnetii, $\mathrm{T}$ cells are implicated to be critical for bacterial clearance, cytokines IFN- $\gamma$ and TNF- $\alpha$ are associated with early control of infection and B cells are important for the prevention of tissue damage $[108,109]$. In pregnant goats, the humoral immunity against $C$. burnetii is strong and can be detected quite early in infection similar to mice. Both $\operatorname{IgM}$ and $\operatorname{IgG}$ antibodies against $C$. burnetii phase I and II antigens are elicited after infection. On the contrary, cellular immune responses are less prominent and only measurable at later infection periods (4 weeks pre-parturition). An increase in systemic IFN- $\gamma$ mRNA levels and production of IFN- $\gamma$ by stimulation of systemic PBMCs are only prominent at later times post-infection. This indicates that the inflammatory cytokines probably do not play a role in preventing $C$. burnetii replication at the beginning of the infection in pregnant goats, as seen in mice $[44,109]$.

Similar to mice and different from goats, a systemic infection of Q fever occurs in humans. During acute infections, a strong cellular host response is provoked which controls the infection by granuloma formation. IFN- $\gamma$ is produced following acute infections which controls the bacterial growth by recruiting monocytes, macrophages and fibroblast at sites of infection and stimulating antimicrobial responses by forming granulomas that effectively enclose sites of $C$. burnetii infection [17]. Thus, cell-mediated immunity in humans is important for control of acute infection and prevention of disease. In contrast, immune responses during chronic $\mathrm{Q}$ fever are ineffective and are associated with reduced granulomas [17]. Elevated levels of interleukin-10 (IL-10) and induced growth of the pathogen in monocytes and macrophages have been found in chronic Q fever patients and are implicated as the pathogens ability to establish a persistent infection [115,116]. IL-10 is a pleiotropic cytokine exhibiting both pro and anti-inflammatory properties. C. burnetii, like other intracellular 
pathogens, may take advantage of the anti-inflammatory properties of IL-10 as a means of avoiding sterilizing immunity and establishing a persistent infection [117-119].

\section{Diagnosis of $\mathbf{Q}$ fever}

Although isolation of $C$. burnetii provides definitive diagnosis, culturing of the pathogen is problematic owing to biosafety standards and extreme infectivity potential of the organism [120]. In humans, laboratory diagnosis of $\mathrm{Q}$ fever is mainly based on serology by enzyme-linked immunosorbent assay (ELISA), indirect immunofluorescence antibody test (IFA) or complement fixation test (CFT). These tests allow detection of phase I and II specific antibodies which are extremely useful in differentiating acute and chronic illness in humans. In acute infections, higher levels of IgM and IgG antibodies against phase II antigens are detected by the third week. During chronic infections elevated IgG antibodies against phase I antigens are uniformly detected $[8,9,121-$ 123]. In animals, C. burnetii infections are usually diagnosed with PCR tests on a wide range of clinical samples (aborted placenta, milk, feces) and serology (CFT and ELISA tests). PCR tests are highly sensitive as it is based on targeting and amplifying multiple copies of genomic IS1111 elements [124]. During the Q fever outbreak in Netherlands diagnosis in animals were conducted both directly and indirectly using PCR tests on IS1111 elements and ELISAs on serum samples [125].

\section{Treatments and vaccines for $Q$ fever}

Presently, antibiotics are the choice of treatment for Q fever in humans. Acute infected patients are usually treated with doxycycline, whereas for chronic infection patients, long term treatment with doxycycline and hydrochloroquine is recommended $[8,9,19,120]$. In animals, the effect of antibiotics is poorly studied and antibiotic treatment with oxytetracycline has shown to be ineffective [126]. The primary method of preventing Q fever is through vaccination of humans and animals. Phase I LPS is a major protective immunogen in human and animal vaccines providing feasible protection against $\mathrm{Q}$ fever. The whole-cell formalin-inactivated vaccine (Q-Vax) with an efficacy of $98 \%$ is considered to be quite safe for humans after a pre-screening skin test [84]. Moreover, a phase I vaccine containing inactivated C. burnetii (Coxevac) has proven to be effective in goats and cattle $[85,127,128]$. However, vaccination with whole-cell materials was shown to induce severe local and occasional systemic reactions [129]. In addition, the requirement of BSL-3 facilities for production of whole cell vaccines makes the process expensive and troublesome. Efforts to overcome this problem by developing a subunit vaccine would offer great potential in the control of Q fever outbreaks as well as limiting the transmission of $C$. burnetii to humans.

\section{Tools and approaches to study the pathogen}

Q fever is known from a very long time but still poorly understood majorly due to the intracellular lifestyle of the bacterium. Development and validation of new and existing tools is required for 
characterization of the pathogen and its interactions with host which will accelerate our understanding of bacterial pathogenesis. Such knowledge will ultimately aid in development of better vaccines, preventing future outbreaks. This section discusses the advantages and critical points of the different tools and approaches required to study C. burnetii.

Host cell free culture: Development of cell-free culture system overcomes the drawbacks encountered when studying the pathogen in cell-based culture or in vivo models. This system allows the molecular characterization of $C$. burnetii by genetic modification techniques like transformation, transposon mutagenesis, gene knock-ins and targeted gene inactivation [51,130-134]. Axenic cultures have also resulted in purification of bacteria free from host cell contaminants $[135,136]$. Such pure bacterial cultures are optimal for genetic and biochemical analyses as well as for vaccine production [78]. Although, cell-free system rescues the bacterium from host, understanding the behavior of $C$. burnetii obtained from this artificial chemical defined media is crucial to extrapolate the results to the in vivo situation. Hence validation of cell-free system with respect to in vivo mimicking systems is important for full appreciation of this advanced culture system.

Enumeration of C. burnetii: Quantification of live C. burnetii is a critical parameter to accurately determine inoculation doses in animal experiments as well as to assess the proliferative capacity of bacterial strains in various culture models. To date, procedures to determine bacterial viability by direct enumeration methods are obtained by focus forming unit (FFU) and colony forming unit assays (CFU). These techniques involve counting of infectious plaques in cells and colonies on agar plates of serially diluted samples respectively. The FFU method involves an indirect immunofluorescent assay using C. burnetii specific monoclonal antibodies to demonstrate infected cells which can be counted microscopically $[52,107,137]$. The CFU method involves a soft agarose overlay method resulting in minute colonies (diameter of $0.05 \mathrm{~mm}$ ) embedded in the semi-solid medium [51]. These direct enumeration methods are tedious, less sensitive and time consuming (6-12 days). Therefore, bacterial quantification is usually conducted by qPCR methods on selected single copy gene $[105,108,109]$. However, qPCR methods fail to discriminate between live/ dead bacterial cells [33]. Hence, it is important to develop a simple and easy method to quantify live amounts of $C$. burnetii cells.

C. burnetii Phase identification: As the ability of C. burnetii to transition between phase I and II forms affects virulence, the ability to quantify the presence of these different forms is crucial for assessing the virulence potential of bacterial cultures. Currently, the most widely used method to identify phase variation is by assessing phase I and II LPS profiles on a SDS-PAGE of extracted LPS samples or digested whole cell lysates,[73,138-142]. This method is feasible but as the antigenic phase transition is accompanied by a chromosomal deletion [86], this approach does not give exact information on which genes are deleted and the mechanism of transition. Additionally, this method also does not give 
a quantitative measurement of the percentage of cells in a bacterial population undergoing the change. Other molecular techniques used are: PCRs on all genes of the LPS encoding regions [143]; genomicsoriented tools like genome hybridization by DNA microarrays [73]; reverse transcriptase-PCR for expression of genes [143]; and sequencing of the O-antigen encoding genomic region [86,143]. These methods are elaborate and tedious; therefore, a more robust and easy to use technique to detect functional deletion of all genes resulting in phase variation would be a comprehensive approach to detect phase variation in C. burnetii bacterial population.

Pathogen Omics approaches: In the last decade advances in deep-sequencing, computational technologies and other genomic technologies have revolutionized the study on viral and bacterial pathogens. For example; the availability of the first whole genome sequence of C. burnetii, has significantly increased our knowledge on the pathogen physiology as well as on putative hostpathogen interactions [54]. Genomic approaches and phylogeographical analysis can be effectively used for source identification in outbreaks and molecular epidemiology, which are usually conducted by more generic genotypic studies [22,28,29,33,144-146]. Available genotyping tools such as MLVA takes advantage of rapid occurring variation of tandemly repetitive DNA sequences $[30,147]$ whereas, MST is based on intergenic region sequencing to differentiate bacterial isolates [148]. As these traditional genotyping methods are based on identification of only a few selected loci on the genome, it lacks sufficient discriminatory power to fully resolve the epidemiology of large outbreaks or to identify chromosomal changes associated with alterations in virulence. Besides, comparative genomic approaches enable the identification of conserved and novel genes as well as Single Nucleotide Polymorphisms (SNPs) to investigate variations between isolates [62,70]. Furthermore, whole-genome transcriptomics approaches can enable functional analysis of pathogens, which will aid in deciphering the mechanism of their interactions with hosts. Therefore, pathogen Omics can be used as a powerful approach to gain new insights into evolution of C. burnetii, to unravel bacterial adaptations to host/ tissue, identification of virulence factors and track mechanisms of pathogenesis contributing to the survival strategy of $C$. burnetii. Knowledge on such virulence signatures may provide a basis for development of next-generation $\mathrm{Q}$ fever vaccines. 


\section{Aim and Outline of Thesis}

The research presented in this thesis focuses on the molecular characterization and pathogenesis of $C$. burnetii (causative agent of $\mathrm{Q}$ fever) strains that were isolated during outbreak period in the Netherlands. These studies were conducted in order to provide knowledge on bacterial virulence, evolution, adaptations under various environmental conditions, identification of factors involved in successful infection and host immune responses to C. burnetii infection. We specifically aimed to identify the virulence factors and mechanisms that contributed to the increased zoonotic potential of the strain associated with the largest Q fever outbreak that occurred from 2007-2010 in The Netherlands. In addition, the thesis describes new tools developed and validated to study C. burnetii.

The main objectives of this thesis were

i. To validate cell-free culture system by comparison of virulence characteristics of C. burnetii strains propagated from cell-based and cell-free cultures

ii. To improve our understanding of pathogenesis of C. burnetii to its microenvironment

iii. To apply comparative genomics approaches in order to:

a. Confirm goats as the source of outbreak in humans during the large $\mathrm{Q}$ fever epidemic in The Netherlands as suggested by epidemiological and genotyping studies

b. To investigate the phylogenetic diversity among various $C$. burnetii outbreak strains

c. To identify potential genetic differences in outbreak strains resulting in hyper-virulence characteristics

iv. To study if differential immune responses to $C$. burnetii strains isolated from various hostorigin could be an explanation of divergent clinical outcomes observed in infected cattle, goats and humans hosts and also to explain the low incidence of human $\mathrm{Q}$ fever outbreaks caused by cattle.

Chapter 1 provides an overview of our current understanding of the Q fever causative agent $C$. burnetii and associated host-pathogen interactions by reviewing relevant scientific literature. This chapter provides a historical perspective regarding discovery of the pathogen and further provides information on the disease and why research into Q fever is crucial. Special emphasis is given to the recent zoonotic impact of this infectious disease in the Netherlands and factors contributing to this unprecedented outbreak of Q fever.

Standard culturing of C. burnetii involves its troublesome propagation inside eukaryotic cells. Whereas since 2009, the introduction of a revolutionary host-cell free culture system, [50] which allows culturing of $C$. burnetii in absence of cells, offers huge advantages in Q fever research. Chapter 2 validates the axenic media by comparing various virulence related characteristics of the cell-free cultured strains with the gold-standard cell-based culture strains in a newly developed mice 
model. Also, a simple and easy indirect enumeration method for assessing viable C. burnetii based on propidium monoazide (PMA) treatment followed by real time PCR was developed and validated with the more laborious direct methods (i.e. counting focus forming and colony forming units). Such a method is crucial for instance in determining inoculation doses of bacteria obtained from different culture systems for animal experiments. Finally, we evaluated the LPS phase of C. burnetii strains using a transcriptomics approach. As deletions of LPS encoding genes are involved in variation from phase I LPS to phase II LPS, we assessed expression of these genes to identify the bacterial phase in cell-based and cell-free cultured strains. The techniques and mice model developed in this study became part of the standard operating procedures for $\mathrm{Q}$ fever research in our facility and were applied in the further studies described in this thesis.

To obtain a better understanding of the pathogen interactions with its host, Chapter 3 describes genome-wide transcriptional profiles of outbreak and reference $C$. burnetii strains assessed in in vivo and different in vitro culture models. Here gene expression differences of bacteria isolated from spleens of experimentally infected mice (in vivo model) and bacteria propagated in cell-based and cell-free cultures (in vitro model) were determined. This aim of this study was to gain a better understanding of the metabolic adaptations and virulence mechanisms employed by the bacteria in its microenvironment by comparing gene expression patterns of the C. burnetii during growth under different natural and more artificial conditions.

Chapter 4 and 5 describe the genome analysis of the C. burnetii outbreak strains isolated during the Q fever outbreak period (2007-2010) in the Netherlands. Using Whole Genome deepSequencing approaches we fully reconstructed the Dutch veterinary outbreak strain genome and a large counterpart of the human outbreak strain. Draft genome sequences of additional C. burnetii strains from different origin (country, species) were reconstructed and analyzed by comparative genomics approaches. Phylogenetic and comparative analyses were performed, to infer relationship between C. burnetii strains and to identify major differences, possible pathogenic adaptation as well as virulence factors in outbreak strains. In addition, the study validates available genotyping tools (based on MLVA and MST methods), which were previously used for $C$. burnetii outbreak investigations.

Next to the pathogen perspective, we also investigated the host immune responses generated by various $C$. burnetii strains. In these studies, the goal was to identify if $C$. burnetii strains originating from various hosts or with different MLVA genotypes have an effect on host immune responses. Chapter 6 describes experiments performed in an in vivo mice infection model, whereas experiments in an in vitro model using healthy human PBMCs are described in Chapter 7. A large collection of $C$. burnetii strains from different host species (goat, sheep, cattle and human) and different MLVA genotypes (CbNL01 and CbNL12) were used in these studies. In the mouse infection model, we measured innate and adaptive immune responses at different time points (Chapter 6). Cytokine profiles generated by healthy human PBMCs upon stimulation with heat-inactivated C. burnetii strains were measured in Chapter 7. 
In Chapter 8 the major findings of this thesis are presented and discussed in the context of available literature. The chapter describes genetic diversity of the Dutch outbreak C. burnetii strains and its links with increased pathogenicity in hosts that could have contributed to the outbreak. We discuss the contributions of pathogen omics experimental approaches in understanding pathogen interactions with host and its potential use in epidemiology and outbreak investigations. Moreover, we also discuss the possible factors resulting in the various disease phenotypes observed in naturally infected animals and the factors that contribute to low risk of cattle and high risk of goats hosts in Q fever outbreaks. Additionally, the relevance of culture and infection models of $C$. burnetii and tools developed to study the pathogen are reviewed. Finally, we discuss the potential utility of the thesis results for disease control and future studies on identification of targets for putative differential diagnostics and $\mathrm{Q}$ fever vaccine candidates. 


\section{References}

1. Derrick EH. "Q" Fever, a New Fever Entity: Clinical Features, Diagnosis and Laboratory Investigation. Rev. Infect. Dis. 1983;5:790-800.

2. Marrie TJ. Historical aspects of Q fever. Q Fever, Volume I: The Disease. Boca Raton, Florida, USA: CRC Press; 1990.

3. Burnet F, Freeman M. Experimental studies on the virus of "Q" fever," Medical Journal of Australia. Med. J. Aust. 1937. Vol. 2, p. 299-305.

4. Davis GE, Cox HR. A filter-passing infectious agent isolated from ticks. I. Isolation from Dermacentor andersonii, reactions in animals, and filtration. Public Health Rep. 1938. Vol. 53, p. 2259-2282.

5. Dyer R. A filter-passing infectious agent isolated from ticks IV. Human Infection. Public Health Rep. 1938. Vol. 53.

6. Centers for Disease Control and Prevention. Bioterrorism agents/diseases. Available from: http://www.bt.cdc.gov/ agent/agentlist-category.asp\#b

7. Coxiella burnetii. Wikipedia website. Available from: https://en.wikipedia.org/wiki/Coxiella_burnetii

8. Maurin M, Raoult D. Q fever. Clin. Microbiol. Rev. 1999;12:518-53.

9. Angelakis E, Raoult D. Q fever. Vet. Microbiol. 2010;140:297-309.

10. Woldehiwet Z. Q fever (coxiellosis): epidemiology and pathogenesis. Res. Vet. Sci. 2004;77:93100 .

11. Roest H-J, van Gelderen B, Dinkla A, Frangoulidis D, van Zijderveld F, Rebel J, et al. Q Fever in Pregnant Goats: Pathogenesis and Excretion of Coxiella burnetii. PLoS ONE. 2012;7:e48949.

12. Norlander L. Q fever epidemiology and pathogenesis. Microbes Infect. Inst. Pasteur. 2000;2:41724.

13. Arricau-Bouvery N, Rodolakis A. Is Q fever an emerging or re-emerging zoonosis? Vet. Res. 2005;36:327-49.

14. Arricau Bouvery N, Souriau A, Lechopier P, Rodolakis A. Experimental Coxiella burnetii infection in pregnant goats: excretion routes. Vet. Res. 2003;34:423-33.

15. Keijmel SP, Delsing CE, Sprong T, Bleijenberg G, van der Meer JW, Knoop H, et al. The Qure study: Q fever fatigue syndrome - response to treatment; a randomized placebo-controlled trial. BMC Infect. Dis. 2013;13:157.

16. Morroy G, Keijmel SP, Delsing CE, Bleijenberg G, Langendam M, Timen A, et al. Fatigue following Acute Q-Fever: A Systematic Literature Review. PLOS ONE. 2016;11:e0155884.

17. Raoult D, Marrie T, Mege J. Natural history and pathophysiology of Q fever. Lancet Infect. Dis. 2005;5:219-26.

18. Mazokopakis EE, Karefilakis CM, Starakis IK. Q fever endocarditis. Infect. Disord. Drug Targets. 2010;10:27-31. 
19. Honarmand H, Honarmand H. Q Fever: An Old but Still a Poorly Understood Disease, Q Fever: An Old but Still a Poorly Understood Disease. Interdiscip. Perspect. Infect. Dis. Interdiscip. Perspect. Infect. Dis. 2012;2012, 2012:e131932.

20. Roest HIJ, Tilburg JJHC, van der Hoek W, Vellema P, van Zijderveld FG, Klaassen CHW, et al. The Q fever epidemic in The Netherlands: history, onset, response and reflection. Epidemiol. Infect. 2011;139:1-12.

21. Karagiannis I, Morroy G, Rietveld A, Horrevorts AM, Hamans M, Francken P, et al. Q fever outbreak in the Netherlands: a preliminary report. Euro Surveill. Bull. Eur. Sur Mal. Transm. Eur. Commun. Dis. Bull. 2007;12:E070809.2.

22. Tilburg JJHC, Rossen JWA, van Hannen EJ, Melchers WJG, Hermans MHA, van de Bovenkamp $\mathrm{J}$, et al. Genotypic diversity of Coxiella burnetii in the 2007-2010 Q fever outbreak episodes in The Netherlands. J. Clin. Microbiol. 2012;50:1076-8.

23. Enserink M. Questions Abound in Q-Fever Explosion in the Netherlands. Science. 2010;327:2667.

24. van der Hoek W, Dijkstra F, Schimmer B, Schneeberger PM, Vellema P, Wijkmans C, et al. Q fever in the Netherlands: an update on the epidemiology and control measures. Euro Surveill. 2010;15.

25. Delsing CE, Kullberg BJ, Bleeker-Rovers CP. Q fever in the Netherlands from 2007 to 2010. Neth. J. Med. 2010;68:382-7.

26. Karagiannis I, Schimmer B, Van Lier A, Timen A, Schneeberger P, Van Rotterdam B, et al. Investigation of a $\mathrm{Q}$ fever outbreak in a rural area of The Netherlands. Epidemiol. Infect. 2009;137:1283-94.

27. Schimmer B, Dijkstra F, Vellema P, Schneeberger PM, Hackert V, ter Schegget R, et al. Sustained intensive transmission of Q fever in the south of the Netherlands, 2009. Euro Surveill. Bull. Eur. Sur Mal. Transm. Eur. Commun. Dis. Bull. 2009;14.

28. Roest HIJ, Ruuls RC, Tilburg JJHC, Nabuurs-Franssen MH, Klaassen CHW, Vellema P, et al. Molecular epidemiology of Coxiella burnetii from ruminants in Q fever outbreak, the Netherlands. Emerg. Infect. Dis. 2011;17:668-75.

29. Tilburg JJHC, Roest H-JIJ, Buffet S, Nabuurs-Franssen MH, Horrevorts AM, Raoult D, et al. Epidemic Genotype of Coxiella burnetii among Goats, Sheep, and Humans in the Netherlands. Emerg. Infect. Dis. 2012;18:887-9.

30. Arricau-Bouvery N, Hauck Y, Bejaoui A, Frangoulidis D, Bodier CC, Souriau A, et al. Molecular characterization of Coxiella burnetii isolates by infrequent restriction site-PCR and MLVA typing. BMC Microbiol. 2006;6:38.

31. Chmielewski T, Sidi-Boumedine K, Duquesne V, Podsiadly E, Thiéry R, TylewskaWierzbanowska S. Molecular epidemiology of Q fever in Poland. Pol. J. Microbiol. Pol. Tow. Mikrobiol. Pol. Soc. Microbiol. 2009;58:9-13.

32. Tilburg JJHC, Roest HJIJ, Nabuurs-Franssen MH, Horrevorts AM, Klaassen CHW. Genotyping reveals the presence of a predominant genotype of Coxiella burnetii in consumer milk products. J. Clin. Microbiol. 2012;50:2156-8.

33. Mori M, Boarbi S, Michel P, Bakinahe R, Rits K, Wattiau P, et al. In Vitro and In Vivo Infectious Potential of Coxiella burnetii: A Study on Belgian Livestock Isolates. PLoS ONE. 2013;8:e67622. 
34. Kampschreur LM, Hagenaars JCJP, Wielders CCH, Elsman P, Lestrade PJ, Koning OHJ, et al. Screening for Coxiella burnetii seroprevalence in chronic Q fever high-risk groups reveals the magnitude of the Dutch Q fever outbreak. Epidemiol. Infect. 2013;141:847-51.

35. Brom RV den, Vellema P. Q fever outbreaks in small ruminants and people in the Netherlands. Small Rumin. Res. 2009;86:74-9.

36. Voth DE, Heinzen RA. Lounging in a lysosome: the intracellular lifestyle of Coxiella burnetii. Cell. Microbiol. 2007;9:829-40.

37. Goren MB, D'Arcy Hart P, Young MR, Armstrong JA. Prevention of phagosome-lysosome fusion in cultured macrophages by sulfatides of Mycobacterium tuberculosis. Proc. Natl. Acad. Sci. U. S. A. $1976 ; 73: 2510-4$.

38. Friis RR. Interaction of L cells and Chlamydia psittaci: entry of the parasite and host responses to its development. J. Bacteriol. 1972;110:706-21.

39. Horwitz MA. The Legionnaires' disease bacterium (Legionella pneumophila) inhibits phagosomelysosome fusion in human monocytes. J. Exp. Med. 1983;158:2108-26.

40. Hale TL, Morris RE, Bonventre PF. Shigella infection of henle intestinal epithelial cells: role of the host cell. Infect. Immun. 1979;24:887-94.

41. Gaillard JL, Berche P, Mounier J, Richard S, Sansonetti P. In vitro model of penetration and intracellular growth of Listeria monocytogenes in the human enterocyte-like cell line Caco-2. Infect. Immun. 1987;55:2822-9.

42. McCaul TF, Williams JC. Developmental cycle of Coxiella burnetii: structure and morphogenesis of vegetative and sporogenic differentiations. J. Bacteriol. 1981;147:1063-76.

43. Hackstadt T, Williams JC. Biochemical stratagem for obligate parasitism of eukaryotic cells by Coxiella burnetii. Proc. Natl. Acad. Sci. U. S. A. 1981;78:3240-4.

44. Roest HIJ, Post J, van Gelderen B, van Zijderveld FG, Rebel JMJ. Q fever in pregnant goats: humoral and cellular immune responses. Vet. Res. 2013;44:67.

45. Baca OG, Akporiaye ET, Aragon AS, Martinez IL, Robles MV, Warner NL. Fate of phase I and phase II Coxiella burnetii in several macrophage-like tumor cell lines. Infect. Immun. 1981;33:258 66.

46. Akporiaye ET, Rowatt JD, Aragon AA, Baca OG. Lysosomal response of a murine macrophagelike cell line persistently infected with Coxiella burnetii. Infect. Immun. 1983;40:1155-62.

47. Baca OG, Scott TO, Akporiaye ET, DeBlassie R, Crissman HA. Cell cycle distribution patterns and generation times of L929 fibroblast cells persistently infected with Coxiella burnetii. Infect. Immun. 1985;47:366-9.

48. Lever MS, Bewley KR, Dowsett B, Lloyd G. In vitro susceptibility of Coxiella burnetii to azithromycin, doxycycline, ciprofloxacin and a range of newer fluoroquinolones. Int. J. Antimicrob. Agents. 2004;24:194-6.

49. Ormsbee RA. The growth of Coxiella burnetii in embryonated eggs. J. Bacteriol. 1952;63:73-86.

50. Omsland A, Cockrell DC, Howe D, Fischer ER, Virtaneva K, Sturdevant DE, et al. Host cell-free growth of the Q fever bacterium Coxiella burnetii. Proc. Natl. Acad. Sci. 2009;106:4430-4. 
51. Omsland A, Beare PA, Hill J, Cockrell DC, Howe D, Hansen B, et al. Isolation from Animal Tissue and Genetic Transformation of Coxiella burnetii Are Facilitated by an Improved Axenic Growth Medium $\nabla$. Appl. Environ. Microbiol. 2011;77:3720-5.

52. Sandoz KM, Sturdevant DE, Hansen B, Heinzen RA. Developmental transitions of Coxiella burnetii grown in axenic media. J. Microbiol. Methods. 2014;96:104-10.

53. Sandoz KM, Beare PA, Cockrell DC, Heinzen RA. A defined axenic medium allows complementation of arginine auxotrophy for genetic transformation of Coxiella burnetii. Appl. Environ. Microbiol. 2016;AEM.00261-16.

54. Seshadri R, Paulsen IT, Eisen JA, Read TD, Nelson KE, Nelson WC, et al. Complete genome sequence of the Q-fever pathogen Coxiella burnetii. Proc. Natl. Acad. Sci. U. S. A. 2003;100:545560.

55. Willems H, Ritter M, Jäger C, Thiele D. Plasmid-homologous sequences in the chromosome of plasmidless Coxiella burnetii Scurry Q217. J. Bacteriol. 1997;179:3293-7.

56. Savinelli EA, Mallavia LP. Comparison of Coxiella burnetii plasmids to homologous chromosomal sequences present in a plasmidless endocarditis-causing isolate. Ann. N. Y. Acad. Sci. 1990;590:523-33.

57. Hendrix LR, Samuel JE, Mallavia LP. Differentiation of Coxiella burnetii isolates by analysis of restriction-endonuclease-digested DNA separated by SDS-PAGE. J. Gen. Microbiol. 1991;137:26976.

58. Cole ST, Eiglmeier K, Parkhill J, James KD, Thomson NR, Wheeler PR, et al. Massive gene decay in the leprosy bacillus. Nature. 2001;409:1007-11.

59. Stephens RS, Kalman S, Lammel C, Fan J, Marathe R, Aravind L, et al. Genome Sequence of an Obligate Intracellular Pathogen of Humans: Chlamydia trachomatis. Science. 1998;282:754-9.

60. Ogata H, Audic S, Renesto-Audiffren P, Fournier P-E, Barbe V, Samson D, et al. Mechanisms of Evolution in Rickettsia conorii and R. prowazekii. Science. 2001;293:2093-8.

61. Parkhill J, Dougan G, James KD, Thomson NR, Pickard D, Wain J, et al. Complete genome sequence of a multiple drug resistant Salmonella enterica serovar Typhi CT18. Nature. 2001;413:84852.

62. Beare PA, Unsworth N, Andoh M, Voth DE, Omsland A, Gilk SD, et al. Comparative genomics reveal extensive transposon-mediated genomic plasticity and diversity among potential effector proteins within the genus Coxiella. Infect. Immun. 2009;77:642-56.

63. Cole ST, Brosch R, Parkhill J, Garnier T, Churcher C, Harris D, et al. Deciphering the biology of Mycobacterium tuberculosis from the complete genome sequence. Nature. 1998;393:537-44.

64. Stinear TP, Seemann T, Harrison PF, Jenkin GA, Davies JK, Johnson PDR, et al. Insights from the complete genome sequence of Mycobacterium marinum on the evolution of Mycobacterium tuberculosis. Genome Res. 2008;18:729-41.

65. Demangel C, Stinear TP, Cole ST. Buruli ulcer: reductive evolution enhances pathogenicity of Mycobacterium ulcerans. Nat. Rev. Microbiol. 2009;7:50-60. 
66. Pupo GM, Lan R, Reeves PR. Multiple independent origins of Shigella clones of Escherichia coli and convergent evolution of many of their characteristics. Proc. Natl. Acad. Sci. U. S. A. 2000;97:10567-72.

67. Maurelli AT, Fernández RE, Bloch CA, Rode CK, Fasano A. "Black holes" and bacterial pathogenicity: A large genomic deletion that enhances the virulence of Shigella spp. and enteroinvasive Escherichia coli. Proc. Natl. Acad. Sci. U. S. A. 1998;95:3943-8.

68. Georgiades K, Raoult D. Genomes of the Most Dangerous Epidemic Bacteria Have a Virulence Repertoire Characterized by Fewer Genes but More Toxin-Antitoxin Modules. PLOS ONE. 2011;6:e17962.

69. D'Amato F, Robert C, Azhar EI, Fournier P-E, Raoult D. Draft Genome Sequence of Coxiella burnetii Strain Cb196, an Agent of Endocarditis in Saudi Arabia. Genome Announc. 2014;2.

70. D'Amato F, Rouli L, Edouard S, Tyczka J, Million M, Robert C, et al. The genome of Coxiella burnetii Z3055, a clone linked to the Netherlands Q fever outbreaks, provides evidence for the role of drift in the emergence of epidemic clones. Comp. Immunol. Microbiol. Infect. Dis. 2014;37:281-8.

71. D’Amato F, Eldin C, Georgiades K, Edouard S, Delerce J, Labas N, et al. Loss of TSS1 in hypervirulent Coxiella burnetii 175, the causative agent of Q fever in French Guiana. Comp. Immunol. Microbiol. Infect. Dis. 2015;41:35-41.

72. Rouli L, Rolain J-M, El Filali A, Robert C, Raoult D. Genome sequence of Coxiella burnetii 109, a doxycycline-resistant clinical isolate. J. Bacteriol. 2012;194:6939.

73. Beare PA, Samuel JE, Howe D, Virtaneva K, Porcella SF, Heinzen RA. Genetic Diversity of the Q Fever Agent, Coxiella burnetii, Assessed by Microarray-Based Whole-Genome Comparisons. J. Bacteriol. 2006;188:2309-24.

74. Sidi-Boumedine K, Ellis RJ, Adam G, Prigent M, Angen $\varnothing$, Aspán A, et al. Draft Genome Sequences of Six Ruminant Coxiella burnetii Isolates of European Origin. Genome Announc. 2014;2:e00285-14.

75. D'amato F, Million M, Edouard S, Delerce J, Robert C, Marrie T, et al. Draft genome sequence of Coxiella burnetii Dog Utad, a strain isolated from a dog-related outbreak of Q fever. New Microbes New Infect. 2014;2:136-7.

76. Walter MC, Öhrman C, Myrtennäs K, Sjödin A, Byström M, Larsson P, et al. Genome sequence of Coxiella burnetii strain Namibia. Stand. Genomic Sci. 2014;9:22.

77. Walter MC, Vincent GA, Stenos J, Graves S, Frangoulidis D. Genome Sequence of Coxiella burnetii Strain AuQ01 (Arandale) from an Australian Patient with Acute Q Fever. Genome Announc. $2014 ; 2$.

78. Hammerl JA, Mertens K, Sprague LD, Hackert VH, Buijs J, Hoebe CJ, et al. First Draft Genome Sequence of a Human Coxiella burnetii Isolate, Originating from the Largest Q Fever Outbreak Ever Reported, the Netherlands, 2007 to 2010. Genome Announc. [Internet]. 2015 [cited 2016 Jan 29];3. Available from: http:/www.ncbi.nlm.nih.gov/pmc/articles/PMC4424315/

79. D'Amato F, Eldin C, Raoult D. The contribution of genomics to the study of Q fever. Future Microbiol. 2016;11:253-72.

80. Moos A, Hackstadt T. Comparative virulence of intra- and interstrain lipopolysaccharide variants of Coxiella burnetii in the guinea pig model. Infect. Immun. 1987;55:1144-50. 
81. Mertens K, Samuel JE. Defense mechanisms against oxidative stress in Coxiella burnetii: adaptation to a unique intracellular niche. Adv. Exp. Med. Biol. 2012;984:39-63.

82. Shannon JG, Howe D, Heinzen RA. Virulent Coxiella burnetii does not activate human dendritic cells: Role of lipopolysaccharide as a shielding molecule. Proc. Natl. Acad. Sci. U. S. A. 2005;102:8722-7.

83. Zhang G, Russell-Lodrigue KE, Andoh M, Zhang Y, Hendrix LR, Samuel JE. Mechanisms of vaccine-induced protective immunity against Coxiella burnetii infection in BALB/c mice. J. Immunol. Baltim. Md 1950. 2007;179:8372-80.

84. Chiu CK, Durrheim DN. A review of the efficacy of human Q fever vaccine registered in Australia. New South Wales Public Health Bull. 2007;18:133-6.

85. Arricau-Bouvery N, Souriau A, Bodier C, Dufour P, Rousset E, Rodolakis A. Effect of vaccination with phase I and phase II Coxiella burnetii vaccines in pregnant goats. Vaccine. 2005;23:4392-402.

86. Hoover TA, Culp DW, Vodkin MH, Williams JC, Thompson HA. Chromosomal DNA deletions explain phenotypic characteristics of two antigenic variants, phase II and RSA 514 (crazy), of the Coxiella burnetii nine mile strain. Infect. Immun. 2002;70:6726-33.

87. Park S-H, Lee H-W, Cao W. Screening of nitrosative stress resistance genes in Coxiella burnetii: Involvement of nucleotide excision repair. Microb. Pathog. 2010;49:323-9.

88. Mertens K, Lantsheer L, Ennis DG, Samuel JE. Constitutive SOS expression and damageinducible AddAB-mediated recombinational repair systems for Coxiella burnetii as potential adaptations for survival within macrophages. Mol. Microbiol. 2008;69:1411-26.

89. Hubber A, Roy CR. Modulation of host cell function by Legionella pneumophila type IV effectors. Annu. Rev. Cell Dev. Biol. 2010;26:261-83.

90. Gilk SD. Role of Lipids in Coxiella burnetii Infection. In: Toman R, Heinzen RA, Samuel JE, Mege J-L, editors. Coxiella Burn. Recent Adv. New Perspect. Res. Q Fever Bact. [Internet]. Springer Netherlands; 2012 [cited 2014 Oct 25]. p. 199-213. Available from: http://link.springer.com/chapter/10.1007/978-94-007-4315-1_10

91. McDonough JA, Newton HJ, Roy CR. Coxiella burnetii secretion systems. Adv. Exp. Med. Biol. 2012;984:171-97.

92. Hill J, Samuel JE. Coxiella burnetii acid phosphatase inhibits the release of reactive oxygen intermediates in polymorphonuclear leukocytes. Infect. Immun. 2011;79:414-20.

93. Wymann MP, Schneiter R. Lipid signalling in disease. Nat. Rev. Mol. Cell Biol. 2008;9:162-76.

94. Stebbins CE, Galán JE. Structural mimicry in bacterial virulence. Nature. 2001;412:701-5.

95. Lührmann A, Nogueira CV, Carey KL, Roy CR. Inhibition of pathogen-induced apoptosis by a Coxiella burnetii type IV effector protein. Proc. Natl. Acad. Sci. 2010;107:18997-9001.

96. Gilk SD, Beare PA, Heinzen RA. Coxiella burnetii expresses a functional $\Delta 24$ sterol reductase. J. Bacteriol. 2010;192:6154-9.

97. Walburger A, Koul A, Ferrari G, Nguyen L, Prescianotto-Baschong C, Huygen K, et al. Protein kinase $\mathrm{G}$ from pathogenic mycobacteria promotes survival within macrophages. Science. 2004;304:1800-4. 
98. Kipreos ET, Pagano M. The F-box protein family. Genome Biol. 2000;1:REVIEWS3002.

99. Angot A, Vergunst A, Genin S, Peeters N. Exploitation of eukaryotic ubiquitin signaling pathways by effectors translocated by bacterial type III and type IV secretion systems. PLoS Pathog. 2007;3:e3.

100. van Schaik EJ, Chen C, Mertens K, Weber MM, Samuel JE. Molecular pathogenesis of the obligate intracellular bacterium Coxiella burnetii. Nat. Rev. Microbiol. 2013;11:561-73.

101. Newton HJ, McDonough JA, Roy CR. Effector Protein Translocation by the Coxiella burnetii Dot/Icm Type IV Secretion System Requires Endocytic Maturation of the Pathogen-Occupied Vacuole. PLoS ONE. 2013;8:e54566.

102. Georgiev M, Afonso A, Neubauer H, Needham H, Thiery R, Rodolakis A, et al. Q fever in humans and farm animals in four European countries, 1982 to 2010. Euro Surveill. Bull. Eur. Sur Mal. Transm. Eur. Commun. Dis. Bull. 2013;18.

103. Guatteo R, Seegers H, Taurel A-F, Joly A, Beaudeau F. Prevalence of Coxiella burnetii infection in domestic ruminants: A critical review. Vet. Microbiol. 2011;149:1-16.

104. Russell-Lodrigue KE, Zhang GQ, McMurray DN, Samuel JE. Clinical and pathologic changes in a guinea pig aerosol challenge model of acute Q fever. Infect. Immun. 2006;74:6085-91.

105. Russell-Lodrigue KE, Andoh M, Poels MWJ, Shive HR, Weeks BR, Zhang GQ, et al. Coxiella burnetii isolates cause genogroup-specific virulence in mouse and guinea pig models of acute $\mathrm{Q}$ fever. Infect. Immun. 2009;77:5640-50.

106. Tamrakar SB, Haluska A, Haas CN, Bartrand TA. Dose-response model of Coxiella burnetii (Q fever). Risk Anal. Off. Publ. Soc. Risk Anal. 2011;31:120-8.

107. Stein A, Louveau C, Lepidi H, Ricci F, Baylac P, Davoust B, et al. Q Fever Pneumonia: Virulence of Coxiella burnetii Pathovars in a Murine Model of Aerosol Infection. Infect. Immun. 2005;73:2469-77.

108. Read AJ, Erickson S, Harmsen AG. Role of CD4+ and CD8+ T Cells in Clearance of Primary Pulmonary Infection with Coxiella burnetii. Infect. Immun. 2010;78:3019-26.

109. Andoh M, Zhang G, Russell-Lodrigue KE, Shive HR, Weeks BR, Samuel JE. T Cells Are Essential for Bacterial Clearance, and Gamma Interferon, Tumor Necrosis Factor Alpha, and B Cells Are Crucial for Disease Development in Coxiella burnetii Infection in Mice. Infect. Immun. 2007;75:3245-55.

110. Marrie TJ, Stein A, Janigan D, Raoult D. Route of infection determines the clinical manifestations of acute Q fever. J. Infect. Dis. 1996;173:484-7.

111. Baumgärtner W, Bachmann S. Histological and immunocytochemical characterization of Coxiella burnetii-associated lesions in the murine uterus and placenta. Infect. Immun. 1992;60:523241.

112. Sánchez J, Souriau A, Buendía AJ, Arricau-Bouvery N, Martínez CM, Salinas J, et al. Experimental Coxiella burnetii infection in pregnant goats: a histopathological and immunohistochemical study. J. Comp. Pathol. 2006;135:108-15.

113. Shannon JG, Heinzen RA. Adaptive immunity to the obligate intracellular pathogen Coxiella burnetii. Immunol. Res. 2009;43:138-48. 
114. Kishimoto RA, Rozmiarek H, Larson EW. Experimental Q fever infection in congenitally athymic nude mice. Infect. Immun. 1978;22:69-71.

115. Capo C, Zaffran Y, Zugun F, Houpikian P, Raoult D, Mege JL. Production of interleukin-10 and transforming growth factor beta by peripheral blood mononuclear cells in $\mathrm{Q}$ fever endocarditis. Infect. Immun. 1996;64:4143-7.

116. Ghigo E, Capo C, Raoult D, Mege JL. Interleukin-10 stimulates Coxiella burnetii replication in human monocytes through tumor necrosis factor down-modulation: role in microbicidal defect of $\mathrm{Q}$ fever. Infect. Immun. 2001;69:2345-52.

117. Aliberti J. Host persistence: exploitation of anti-inflammatory pathways by Toxoplasma gondii. Nat. Rev. Immunol. 2005;5:162-70.

118. Nylén S, Sacks D. Interleukin-10 and the pathogenesis of human visceral leishmaniasis. Trends Immunol. 2007;28:378-84.

119. Tufariello JM, Chan J, Flynn JL. Latent tuberculosis: mechanisms of host and bacillus that contribute to persistent infection. Lancet Infect. Dis. 2003;3:578-90.

120. Hartzell JD, Wood-Morris RN, Martinez LJ, Trotta RF. Q Fever: Epidemiology, Diagnosis, and Treatment. Mayo Clin. Proc. 2008;83:574-9.

121. Kampschreur LM, Oosterheert JJ, Koop AMC, Wegdam-Blans MCA, Delsing CE, BleekerRovers $\mathrm{CP}$, et al. Microbiological challenges in the diagnosis of chronic Q fever. Clin. Vaccine Immunol. CVI. 2012;19:787-90.

122. Jager MM, Weers-Pothoff G, Hermans MHA, Meekelenkamp JCE, Schellekens JJA, Renders NHM, et al. Evaluation of a Diagnostic Algorithm for Acute Q Fever in an Outbreak Setting ${ }^{\nabla}$. Clin. Vaccine Immunol. CVI. 2011;18:963-8.

123. Fournier P-E, Marrie TJ, Raoult D. Diagnosis of Q Fever. J. Clin. Microbiol. 1998;36:1823-34.

124. Klee SR, Tyczka J, Ellerbrok H, Franz T, Linke S, Baljer G, et al. Highly sensitive real-time PCR for specific detection and quantification of Coxiella burnetii. BMC Microbiol. 2006;6:2.

125. Sidi-Boumedine K, Rousset E, Henning K, Ziller M, Niemczuck K, Roest H, et al. Development of harmonised schemes for the monitoring and reporting of Q-fever in animals in the European Union. EFSA Support. Publ. 2010;7:n/a-n/a.

126. SZYMAŃSKA-CZERWIŃSKA M, NIEMCZUK K. Evaluation of the Effectiveness of Q Fever Treatment with Oxytetracycline. Bull. Vet. Inst. Pulawy. 2013. p. 513-7.

127. Hogerwerf L, van den Brom R, Roest HIJ, Bouma A, Vellema P, Pieterse M, et al. Reduction of Coxiella burnetii prevalence by vaccination of goats and sheep, The Netherlands. Emerg. Infect. Dis. 2011;17:379-86.

128. Guatteo R, Seegers H, Joly A, Beaudeau F. Prevention of Coxiella burnetii shedding in infected dairy herds using a phase I C. burnetii inactivated vaccine. Vaccine. 2008;26:4320-8.

129. Waag DM, Kende M, Damrow TA, Wood OL, Williams JC. Injection of inactivated phase I Coxiella burnetii increases non-specific resistance to infection and stimulates lymphokine production in mice. Ann. N. Y. Acad. Sci. 1990;590:203-14. 
130. Beare P, Heinzen R. Gene Inactivation in Coxiella burnetii. In: Vergunst AC, O'Callaghan D, editors. Host-Bact. Interact. [Internet]. Springer New York; 2014 [cited 2016 Aug 8]. p. 329-45. Available from: http://dx.doi.org/10.1007/978-1-4939-1261-2_19

131. Beare PA. Genetic Manipulation of Coxiella burnetii. In: Toman R, Heinzen RA, Samuel JE, Mege J-L, editors. Coxiella Burn. Recent Adv. New Perspect. Res. Q Fever Bact. [Internet]. Springer Netherlands; 2012 [cited 2016 Aug 8]. p. 249-71. Available from: http://link.springer.com/chapter/10.1007/978-94-007-4315-1_13

132. Beare PA, Larson CL, Gilk SD, Heinzen RA. Two Systems for Targeted Gene Deletion in Coxiella burnetii. Appl. Environ. Microbiol. 2012;78:4580-9.

133. Voth DE, Beare PA, Howe D, Sharma UM, Samoilis G, Cockrell DC, et al. The Coxiella burnetii Cryptic Plasmid Is Enriched in Genes Encoding Type IV Secretion System Substrates. J. Bacteriol. 2011;193:1493-503.

134. Chen C, Banga S, Mertens K, Weber MM, Gorbaslieva I, Tan Y, et al. Large-scale identification and translocation of type IV secretion substrates by Coxiella burnetii. Proc. Natl. Acad. Sci. U. S. A. 2010;107:21755-60.

135. Williams JC, Peacock MG, McCaul TF. Immunological and biological characterization of Coxiella burnetii, phases I and II, separated from host components. Infect. Immun. 1981;32:840-51.

136. Cockrell DC, Beare PA, Fischer ER, Howe D, Heinzen RA. A method for purifying obligate intracellular Coxiella burnetii that employs digitonin lysis of host cells. J. Microbiol. Methods. 2008;72:321-5.

137. Howe D, Barrows LF, Lindstrom NM, Heinzen RA. Nitric Oxide Inhibits Coxiella burnetii Replication and Parasitophorous Vacuole Maturation. Infect. Immun. 2002;70:5140-7.

138. Baca OG, Martinez IL, Aragón AS, Klassen D. Isolation and partial characterization of a lipopolysaccharide from phase II Coxiella burnetii. Can. J. Microbiol. 1980;26:819-26.

139. Schramek S, Mayer H. Different sugar compositions of lipopolysaccharides isolated from phase I and pure phase II cells of Coxiella burnetii. Infect. Immun. 1982;38:53-7.

140. Amano K, Williams JC, McCaul TF, Peacock MG. Biochemical and immunological properties of Coxiella burnetii cell wall and peptidoglycan-protein complex fractions. J. Bacteriol. 1984;160:982-8.

141. Mayer H, Radziejewska-Lebrecht J, Schramek S. Chemical and immunochemical studies on lipopolysaccharides of Coxiella burnetii phase I and phase II. Adv. Exp. Med. Biol. 1988;228:577-91.

142. Kersh GJ, Oliver LD, Self JS, Fitzpatrick KA, Massung RF. Virulence of pathogenic Coxiella burnetii strains after growth in the absence of host cells. Vector Borne Zoonotic Dis. Larchmt. N. $2011 ; 11: 1433-8$.

143. Denison AM, Massung RF, Thompson HA. Analysis of the O-antigen biosynthesis regions of phase II isolates of Coxiella burnetii. FEMS Microbiol. Lett. 2007;267:102-7.

144. Santos AS, Tilburg JJHC, Botelho A, Barahona MJ, Núncio MS, Nabuurs-Franssen MH, et al. Genotypic diversity of clinical Coxiella burnetii isolates from Portugal based on MST and MLVA typing. Int. J. Med. Microbiol. IJMM. 2012;302:253-6.

145. Sulyok KM, Hornok S, Abichu G, Erdélyi K, Gyuranecz M. Identification of Novel Coxiella burnetii Genotypes from Ethiopian Ticks. PLOS ONE. 2014;9:e113213. 
146. Sulyok KM, Kreizinger Z, Hornstra HM, Pearson T, Szigeti A, Dán Á, et al. Genotyping of Coxiella burnetiifrom domestic ruminants and human in Hungary: indication of various genotypes. BMC Vet. Res. 2014;10:107.

147. Svraka S, Toman R, Skultety L, Slaba K, Homan WL. Establishment of a genotyping scheme for Coxiella burnetii. FEMS Microbiol. Lett. 2006;254:268-74.

148. Glazunova O, Roux V, Freylikman O, Sekeyova Z, Fournous G, Tyczka J, et al. Coxiella burnetii Genotyping. Emerg. Infect. Dis. 2005;11:1211-7. 



\section{Chapter 2}

\section{Cell-free propagation of Coxiella burnetii does not affect its relative virulence}

Runa Kuley, Hilde E. Smith, Dimitrios Frangoulidis, Mari A. Smits, Hendrik I. Jan Roest and Alex Bossers 


\begin{abstract}
Q fever is caused by the obligate intracellular bacterium Coxiella burnetii. In vitro growth of the bacterium is usually limited to viable eukaryotic host cells imposing experimental constraints for molecular studies, such as the identification and characterization of major virulence factors. Studies of pathogenicity may benefit from the recent development of an extracellular growth medium for $C$. burnetii. However, it is crucial to investigate the consistency of the virulence phenotype of strains propagated by the two fundamentally different culturing systems. In the present study, we assessed the viability of $C$. burnetii and the lipopolysaccharide (LPS) encoding region of the bacteria in both culture systems as indirect but key parameters to the infection potential of C. burnetii. Propidium monoazide (PMA) treatment-based real-time PCR was used for enumeration of viable C. burnetii which were validated by fluorescent infectious focus forming unit counting assays. Furthermore, RNA isolated from $C$. burnetii propagated in both the culture systems was examined for LPS-related gene expression. All thus far known LPS-related genes were found to be expressed in early passages in both culturing systems indicating the presence of predominantly the phase I form of $C$. burnetii. Finally, we used immune-competent mice to provide direct evidence, that the relative virulence of different $C$. burnetii strains is essentially the same for both axenic and cell-based methods of propagation.
\end{abstract}




\section{Introduction}

Coxiella burnetii, the etiological agent of $\mathrm{Q}$ fever, is an obligate intracellular bacterium that multiplies within a modified phagolysosome of eukaryotic cells. Q fever is primarily a zoonotic infection that is transmitted via inhalation of contaminated aerosols associated with domestic livestock operations [1]. In humans $\mathrm{Q}$ fever manifests as acute and chronic infections. Acute infection symptoms commonly include fever, pneumonia or hepatitis [2,3] whereas persistent infections lead to chronic disease commonly presenting as endocarditis, which is difficult to treat with antibiotics [4]. A large outbreak occurred in the Netherlands during the years 2007-2010 where more than 40,000 people were assumed to be infected [5-7]. The causes for the outbreak are not fully understood and one of the main reasons speculated were the hyper virulent behavior of the circulating strains which might have resulted in the increased zoonotic potential $[6,8,9]$. Unfortunately, more accessible cell-free cultivation $[10,11]$ of $C$. burnetii to be used in, for instance genetic modification or sample multiplication studies were not linked to virulence measurements yet. Validation of such tools assists in improved understanding of the pathogenesis of $C$. burnetii, which in turn may contribute to a more efficient control of the disease.

The obligate intracellular nature of $C$. burnetii imposes several obstacles and makes it to be largely experimentally intractable to use molecular-genetic approaches aiming at a better understanding of its pathogenesis and virulence factors. Studying these factors of $C$. burnetii may benefit from the recent development of an extracellular growth medium [10,11]. However, it is crucial to know whether the viability of $C$. burnetii strains, as well as aspects of phase variation and virulence characteristics is not affected by the two different culturing systems. So far procedures to determine the viability of the bacteria based on direct enumeration methods (for instance colony counting) are not easily achievable for $C$. burnetii, although this is needed to accurately determine inoculation doses in animal experiments. Less often the viability of bacteria is measured by counting the infectious plaques/colonies in cells infected with serially diluted samples, but this method is tedious, less sensitive and limited to cell-based culture systems [12]. Therefore, quantification of bacteria is usually done by total DNA qPCR methods on selected single-copy genes [13-15]. Nevertheless, qPCR method fails to differentiate between live and dead cells showing the necessity to develop easier methods to quantify live amounts of $C$. burnetii.

The production of a structurally and antigenetically unique lipopolysaccharide (LPS) molecule is an important feature in C. burnetii as LPS is one of the important virulence factors of the organism. The presence or absence of LPS also largely correlates with the phase-variation phenomenon in $C$. burnetii [16]. From the two phases, phase I is characterized by the presence of full LPS and is typically the virulent variant whereas phase II has a truncated LPS and usually considered avirulent [17]. All the LPS coding genes are located in a $38 \mathrm{~kb}$ region in the C. burnetii genome and it has been observed that chromosomal deletions in this region result in antigenic phase variation from phase I to phase II $[18,19]$. The shift from phase I to phase II occurs usually due to repeated passages of the 
strains in cultures or embryonated eggs $[17,20]$, but the molecular mechanisms influencing the LPS modifications still remain unclear.

Propagation of $C$. burnetii in cell-based cultures is considered the gold standard for in vitro growth. The development of an axenic (cell-free) growth medium for C. burnetii has greatly facilitated genetic manipulation and the isolation of single colonies on solid medium, which is not possible with cell-based cultivation methods [10,11,21-24]. However, the cell-free system is an enhanced artificial environment for $C$. burnetii cultivation in comparison to cell-based propagation, which closely mimics in vivo infection conditions. Although C. burnetii grown in cell-free medium exhibited developmental forms characteristic of in vivo grown organisms and are infective [11], it is not known whether they have altered virulence characteristics compared to C. burnetii grown in cell-based culture.

Within the scope of virulence factor characterization, the aim of the present study was to investigate, whether propagation in two different in vitro culturing systems affects the viability, phase variation and relative virulence of $C$. burnetii strains. To assess viability of the $C$. burnetii strains, we used propidium monoazide (PMA) dye after comparing it with ethidium monoazide (EMA) dye for selective PCR amplification of DNA from viable bacterial cells [25] as well as with fluorescent infectious focus forming units counting (FFU) [12,26,27] and counting colony forming units (CFU) [10]. To determine the phase of the strains, expression of LPS coding genes was studied by using microarrays on RNA isolated from low and high passaged C. burnetii strains. The level of gene expression served as an indicator to predict the functional state of the genes present in the LPS coding region. The gene expression studies were further validated by using raw next generation sequencing reads to assess for any potential deletions of genes involved in LPS synthesis. Ultimately, the relative virulent phenotype of $C$. burnetii strains was determined by experimental infection using an immunecompetent mice model [28-31]. This study provides evidence that cell-free propagation of C. burnetii does not affect its viability, phase variation and relative virulence as seen by mouse virulence bioassay.

\section{Materials and methods}

\section{Animals}

Two independent animal experiments were conducted using Specific-Pathogen-Free Swiss female OF1 mice (Charles River, l'Arbresle, France). For Experiment 1 (E1) and Experiment 2 (E2), 6 and 7 week old mice were used respectively. The mice were housed under sterile conditions in biosafety level 3 facilities and acclimatized for one week before experimental infection. Each group was housed separately and consisted of 10 mice.

\section{Ethics statement}

Animal experiments were approved by the animal experiment commission of the Central Veterinary Institute of Wageningen UR, and conducted in accordance with the Dutch regulations on animal 
experimentation (Registration numbers: 2010011.b, 2012126.a). Humane end points were defined prior to the experiments and all possible measures were taken to minimize animal suffering.

\section{C. burnetii strains and cultures}

In this study the isolates X09003262-001 (3262); X08014160-001 (601) and X08014160-002 (602) were used. These isolates were primarily isolated from the placentas of goats that aborted during the $\mathrm{Q}$ fever outbreak in the Netherlands (2007-2010) [5]. These 3 isolates were molecular typed as 2 genetically different strains (602 and 3262 similar molecular MLVA genotype) [5] and were initially propagated in Buffalo Green Monkey (BGM) cells (European Collection of Cell Cultures, Salisbury, U.K) without antibiotics as described previously [30]. Two additional strains included in the study were Nine-Mile RSA 493 (NM) and Scurry Q217 (scurry) provided by D. Frangoulidis (Bundeswehr Institute of Microbiology, Munich) and were initially propagated in the same way. The stock cultures of all the strains propagated in $\mathrm{BGM}$ cells were stored at $-80^{\circ} \mathrm{C}$. Prior to experimentally infecting mice, the strains were propagated for a few passages ( $\mathrm{n}<4 \mathrm{x}$ times) in cell based and cell free culture systems in order to adapt the strains to the new (cell free) culture system [10] and to synchronize the passage numbers of the strains.

\section{Viability PCR and quantification of $C$. burnetii}

To determine viability of $C$. burnetii, PMA and EMA dyes were compared on strains cultured from cell-based and cell-free culture system. The protocol is essentially described before [25], where EMA was used to a final concentration of $100 \mu \mathrm{M}$ to assess the viability of C. burnetii. A similar final concentration of $100 \mu \mathrm{M}$ of EMA and PMA (Geniul, Spain) was used in this study. Briefly, the dyes were added to the samples in opaque tubes and incubated in dark for $30 \mathrm{~min}$ at $4^{\circ} \mathrm{C}$ and vortexed regularly. The samples were transferred to high transparent tubes and were photo-activated for 30 minutes using PhAST blue system (Genuil, Spain). DNA was isolated from the samples by using NucliSENS easyMag machine (BioMérieux, USA) as per manufacture instructions. One tenth of the DNA was used as a template for detection and quantification of number of C. burnetii by using a TaqMan-based real-time PCR targeting the single copy gene (CBU_0407a, gene bank number AY502846) encoding a C. burnetii specific hypothetical protein as previously published [30]. A standard curve was generated by using ADIAVET COX Positive control (Adiagene, Marcy l'Étoile, France) according to the manufacturer's instructions.

\section{Fluorescent infectious focus forming unit's measurements}

Viability PCRs were validated by a direct enumeration method using microscopic counting of fluorescent infectious focus forming units (FFU). The viable number of bacterial cells were established by inoculating serial dilutions of bacterial cells $\left(10^{-1}\right.$ to $\left.10^{-9}\right)$ from both culture systems on confluent monolayers of BGM cells in 24 well tissue culture plates (Costar, Corning, USA) containing 
$15 \mathrm{~mm}$ coverslips (Menzel $\mathrm{GmbH}$, Germany). After 24 hours incubation at $37^{\circ} \mathrm{C}$ in $5 \% \mathrm{CO}_{2}$, infected cells were washed twice and incubated for 6 days. Infected cells were demonstrated by indirect immunofluorescence assay using C. burnetii specific monoclonal antibody (MAB313-oregon green, Squarix). Number of viable bacteria in the inoculum was determined from the last dilution which resulted in at least one infected cell [12]. To further confirm the FFU counts, CFU counts were also performed with the same bacterial dilutions used for FFU on solid agar plates as mentioned previously [10].

\section{RNA preparation and microarray analysis}

Total RNA was isolated from C. burnetii strains by using Direct-zol RNA Miniprep Kit (Zymo Research, Irvine, USA) as per manufacturer's instructions. Poly-A tails were tagged to bacterial total RNA (500 ng) by using Poly-A Polymerase Tailing kit (Epicenter Illumina company, Madison, USA). This RNA was used as starting material for Agilent's protocols for amplification, labelling, hybridization, washing and scanning of the C. burnetii microarrays. The C. burnetii custom microarray consisted of user-designed probes (https://earray.chem.agilent.com/earray) covering the complete LPS-gene repertoire of several C. burnetii strains (NCBI, Accession numbers: AE016828.2, CP000733.1, CP001020.1, CP001019.1, CP001021.1) and were synthesized by Agilent Technologies (Santa Clara, CA, USA). Data was obtained through Agilent's feature extraction software (Version 10.7.3.1) and analyzed by using GeneSpring GX software (Agilent Technologies).

\section{Genome coverage analysis}

Total DNA from high passage C. burnetii strains ( $\mathrm{n}>30 \mathrm{x}$ times) propagated in cell-free culture was isolated by phenol-chloroform method [32] after overnight incubation with ATL lysis buffer and proteinase K (QIAGEN, Hilden, Germany). Whole genome sequencing was performed using $250 \mathrm{bp}$ paired-end sequencing libraries (Nextera TAG-mentation sequencing kits, Epicentre, Madison, USA) on an Illumina MiSeq sequencer. High quality paired end reads were mapped against the reference NM RSA493 genome available from the NCBI database (Accession number: AE016828.2) using Bowtie2 alignment tool [33]. The average coverage and standard deviation (excluding repetitive regions) were obtained for the whole genome using a sliding-window approach and were used to calculate the coverage of genes involved in LPS synthesis (CBU0676 to CBU0706). Coverage below the minus 2 SD interval was considered as a significantly deleted gene. Apart from genome analysis, the protein profiles of low and high passaged 602 strain were also analyzed by SDS-PAGE using the whole cell lysates, as described previously [34]. The method was basically the same except for staining of proteins with SYPRO Orange dye (Molecular probes) and visualizing the protein profiles with a Typhoon Trio imager and ImageQuant 5.2 software (Molecular Dynamics). 


\section{Infection mice and collection of tissues}

Two independent infection experiments were performed where 10 Swiss OF1 mice per strain were inoculated intraperitoneally with $0.2 \mathrm{ml}$ suspension of $C$. burnetii in phosphate buffer saline (PBS) in order that each mouse receives a dose of $10^{5}$ genome copy equivalents as determined by quantitative PCR. PBS was used for the negative controls. The inocula used to infect mice were tested for number of viable cells after the infection experiments by using PMA-PCR. The samples were freeze thawed once and similar percentages (55-65\%) of live bacterial cells were found in the inocula. All the strains from both culture systems were found to be in phase I as measured by expression profiles of LPS encoding genes using a microarray. One week after inoculation the mice were euthanized, weighed and their spleen were aseptically removed, weighed and frozen at $-80^{\circ} \mathrm{C}$. Weights were used for determining relative spleen weights (means of spleen weight/body weight (\%)).

\section{Measurements of bacterial loads from spleens of mice}

To quantify the number of $C$. burnetii in mice spleens, DNA was extracted from the spleen by using the DNeasy Blood and Tissue Kit (QIAGEN, Hilden, Germany). Briefly, the spleen was homogenized after addition of PBS. About $200 \mu 1$ suspension was taken and added to tissue lysis buffer (buffer ATL) and proteinase K. DNA was extracted from this suspension according to the protocol for gramnegative bacteria as recommended by the manufacturer.

The DNA was used as a template for detection and quantification of number of $C$. burnetii by using a TaqMan-based real-time PCR as explained above. The bacterial genome copy number from PCR was $\log$ transformed and determined as the mean $\log 2$ values per gram spleen.

\section{Statistical analysis}

Samples and sample-derived data from each mouse were kept separate for all treatments and analysis. Data from viability of PMA treated samples, normalized expression of LPS genes and relative virulence from groups of mice are expressed as means \pm standard deviation and compared by independent two tailed student $t$-tests. Differences were considered significant at a $p$ value of $<0.01$.

\section{Results}

\section{Evaluation and comparison of PMA and EMA dyes for the $C$. burnetii live/dead assay}

Propidium monoazide (PMA) and Ethidium monoazide (EMA) were utilized to selectively allow realtime PCR amplification of target DNA from viable C. burnetii cells. The basic principle involves selective penetration of these dyes only into dead bacterial cells with compromised membrane integrity but not into the live cells with intact membranes. Once inside the cells, the dyes cross link with DNA upon exposure to bright light and inhibits its amplification by PCR [35]. In the present study serially diluted $C$. burnetii (NM strain) containing approximately $10^{6}$ cells $/ \mathrm{ml}$ from cell-based and cell-free culture matrix were treated with EMA and PMA. The results show a clear difference 
A

$\square$ Cell culture

$\square$ Cell-free culture

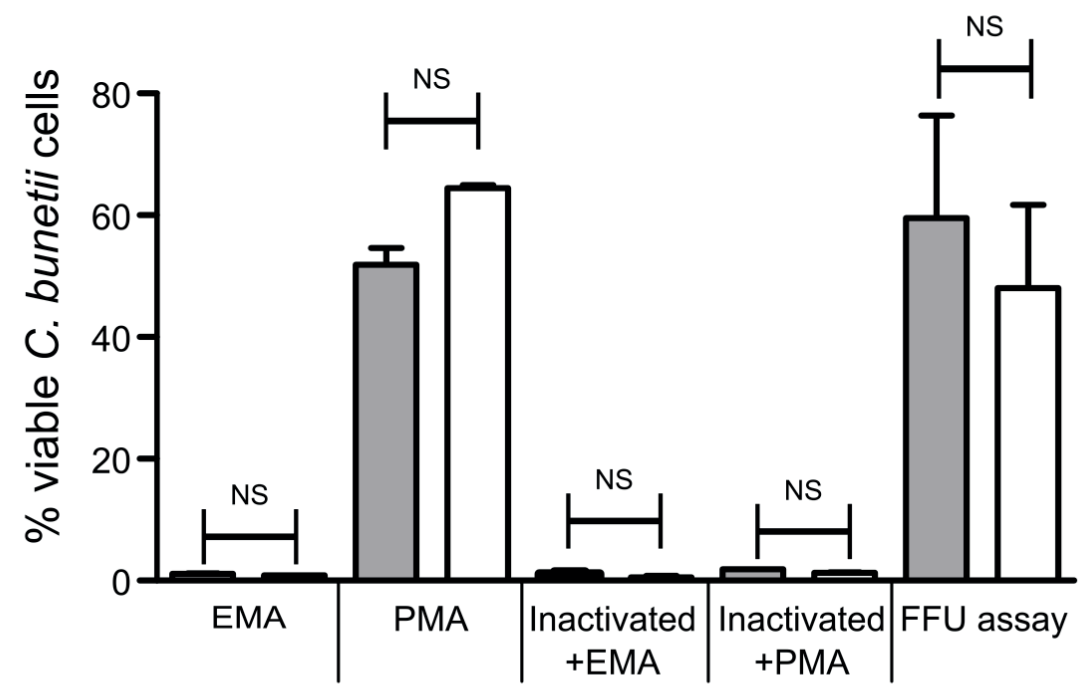

B

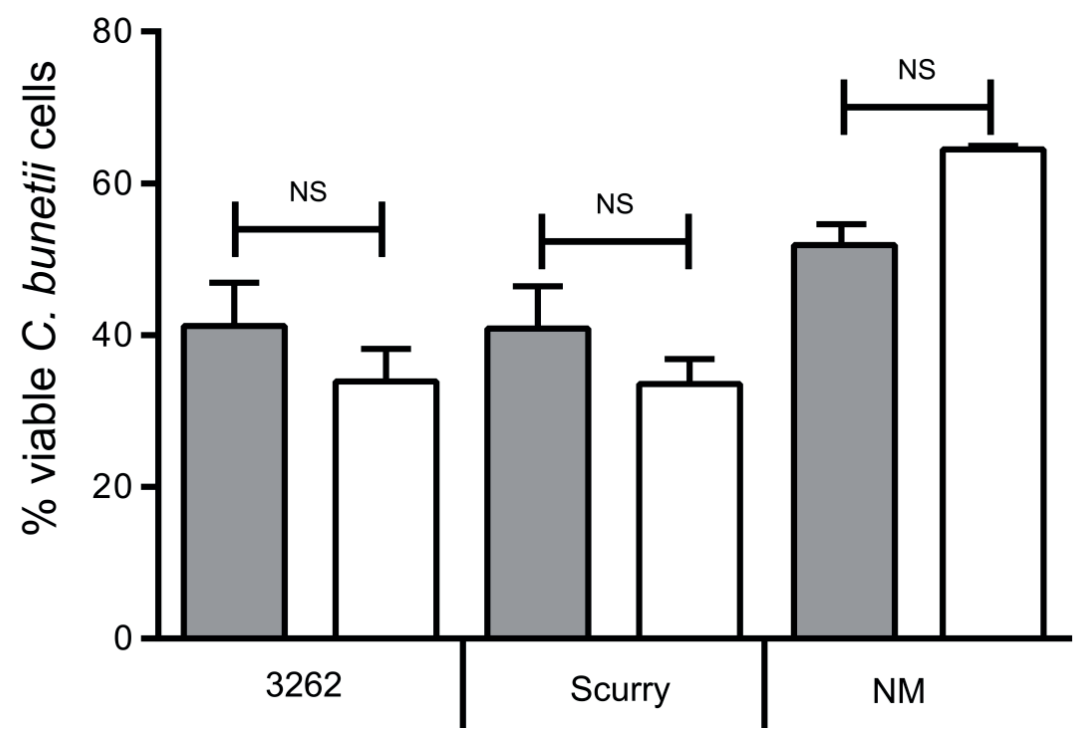

Figure 1. Viability PCR of C. burnetii on the single copy gene in cell-based and cell-free matrix. A) Comparison of PMA and EMA PCR with limited dilution FFU assays. PMA and EMA measurements were performed on normal and heat-inactivated (dead) cells of $C$. burnetii. FFU assays were performed to validate PMA-PCR measurements. B) C. burnetii strains were exposed with PMA and the genomic DNA yield was expressed as a percentage of alive cells corresponding to non-PMA-treated sample. The error bars represent standard deviations from two independent replicates. NS indicates $p$-value $>0.01$. 
between the two dyes. In PMA treated samples $50-60 \%$ of alive cells were detected, whereas in EMA treated samples only few cells (1-2\%) were detected to be viable based on the DNA amplification compared to untreated samples (Fig. 1A). We concluded that, PMA dye selectively entered dead cells that allowed efficient amplification of live C. burnetii, whereas EMA inhibits DNA amplification of almost all cells. To validate the efficiency of PMA-PCR, limiting dilution FFU assays were conducted to measure the number of viable cells based on the last dilution that resulted in at least one infected cell. Similar amounts of viable bacteria from both the culture systems were measured as seen by PMA-PCR (Fig. 1A). Furthermore, CFU counts [10] also confirmed the FFU counts, where the last dilution resulting in colonies corresponded with the same dilution which resulted in at least one infected cell in FFU assay (data not shown). To test the potential in discriminating live and dead cells by these dyes, the samples were subjected to heat treatment at $99^{\circ} \mathrm{C}$ for $30 \mathrm{~min}$. Very high signal reduction was seen in samples both treated with EMA and PMA with $>99 \%$ decrease in DNA amplification. To further test the reproducibility of this technique, different strains from cell-based and cell-free culture (3262, Scurry and NM) were tested with PMA followed by qPCR. In PMA treated samples $30-65 \%$ of live cells were detected based on the DNA amplification (Fig. 1B) with respect to non-PMA treated samples. No significant differences were seen between culture systems with respect to viability of cells (Fig. 1B).

\section{Gene expression in LPS coding genes of $C$. burnetii}

Within the $38 \mathrm{~kb}$ region of $C$. burnetii strains the junctions of large, nested deletions are located which can lead to phase variation [18]. We first tried to find evidence for the presence or absence of these deletions using a PCR approach (data not shown) targeting on multiple locations in this region. By this approach, we did not find any clear evidence for the presence of deletions as would be seen in a phase II variant [18]. To obtain additional evidence for the absence of LPS gene deletions, we subsequently used a microarray approach to measure the expression level of genes in this region in cells grown in both cell-based and cell-free culture systems (Fig. 2A). The average gene expression was normalized with expression of the housekeeping gene $r p o B$ [36] of $C$. burnetii and was plotted for the 602 strain as a representative example. Here, a measurable expression indicates the presence of transcribed RNA from genes located in the $38 \mathrm{~kb}$ region, while no expression points towards a possible loss of function due to regulation or deletion events. The comparison of normalized LPS gene expressions from strain 602 shows similar expression levels for all genes in the LPS encoding region in both cell-based and cell-free cultured strains. This supports the earlier conclusion that for both culturing systems no deletion in the LPS region could be detected. A similar trend of LPS expression is seen in other strains (data not shown) that were used in the virulence bioassay of mice as described in this study, indicating that the majority of cells as cultured by low passage-number in the cell-based and cell-free system are in phase I. In contrast, high passaged 602 strain from cell-free culture showed a very low expression of LPS genes at the beginning of the LPS encoding region (CBU0676 to CBU0697) and high expression 


\section{$\rightarrow$ Cell culture $\rightarrow$ Cell-free culture $\rightarrow$ Cell-free culture high passage}
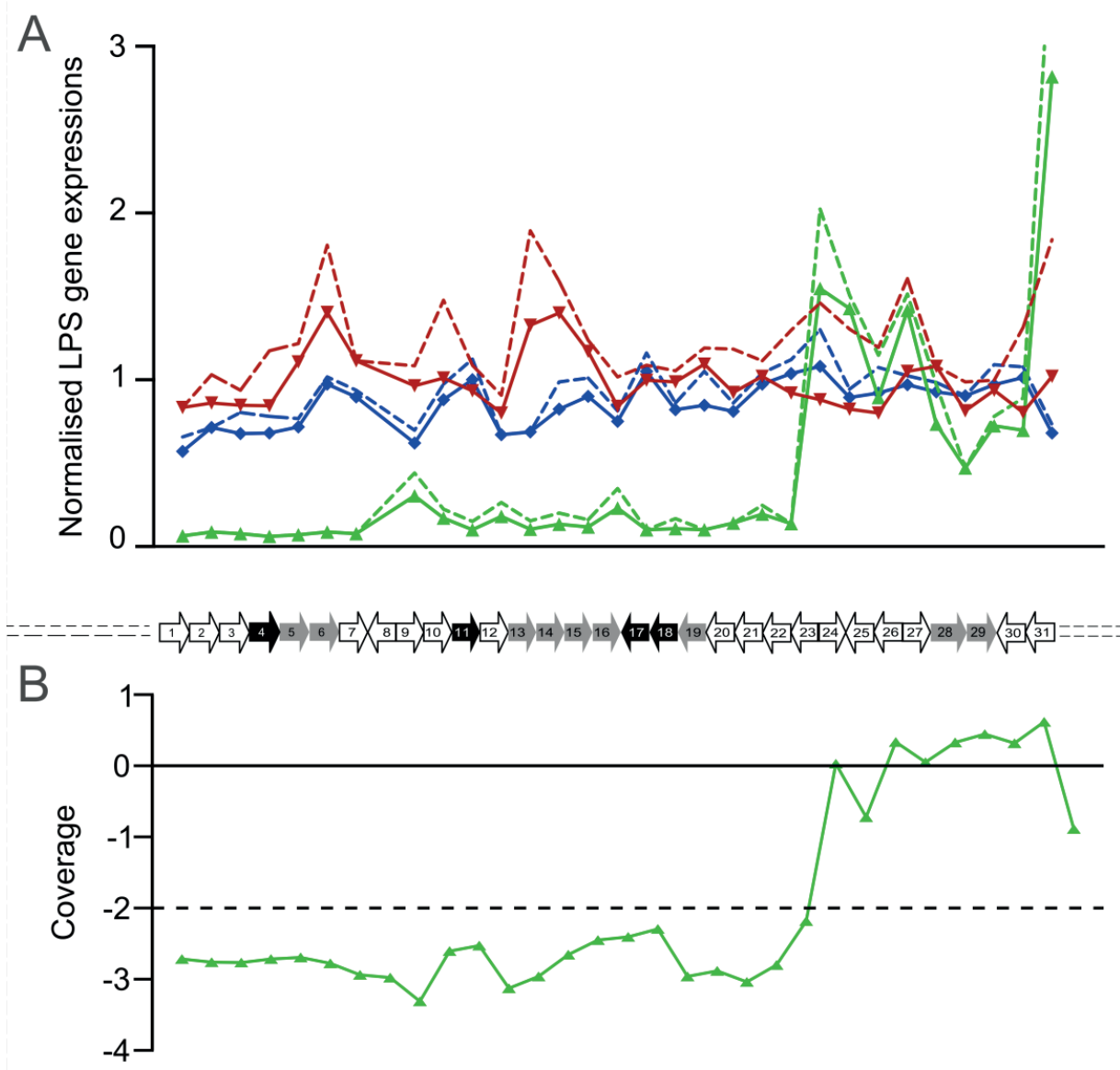

Figure 2. Normalized LPS gene expression profiles and genome coverage of C. burnetii 602 strain as a representative example. A) Here average transcript signal per gene was normalized with the average transcript signal of housekeeping gene $r p o B$ of $C$. burnetii. The normalized gene expressions are plotted for 602 low passage strain in cell-based and cell-free culture systems and 602 high passaged cell-free culture strain. The dashed lines represent standard deviations from two independent replicates. B) Genomic coverage of genes involved in LPS synthesis of a high passaged cell-free culture 602 strain. Coverage below the minus 2 SD interval (dashed line) was considered as a significantly deleted gene. The $\mathrm{x}$-axis for both the A and B graphs are shown as a scaled mini-map of $C$. burnetii LPS encoding genes. Each data point represents to its corresponding gene (CBU0676 to CBU0706) labelled as 1 to 31 genes in the gene map. The genes indicated in grey are hypothesized to be involved O-antigen synthesis and export. Genes indicated in black are hypothesized to be involved in other LPS synthesis steps and carbohydrate metabolism. 
of the genes towards the end (CBU0698 to CBU0706) (Fig. 2A). Comparing the regulation of LPS encoding genes of high passaged cell-free culture with low passaged cell-based and cell-free culture strains showed a significant $(\mathrm{p}<0.01)$ down regulation of CBU0676 to CBU0697 genes with a minimum fold change $<4$ (data not shown). These significant difference between the higher passaged cells $(\mathrm{n}>30 \mathrm{x}$ times) and the lower passaged cells $(\mathrm{n}<10 \mathrm{x}$ times) shows loss of function of the genes (CBU0676 to CBU0697) at the beginning of the LPS cluster and a consecutive variation from phase I to phase II in a significant proportion of the cell population.

\section{Deletion of genes involved in LPS formation by whole genome sequence analysis}

The low expression of genes involved in LPS synthesis in the high passaged C. burnetii strain were further analyzed for any deletions by whole genome sequencing. The coverage of reads for LPS encoding genes (CBU0676 to CBU0697) were significantly lower than the genes present at the end (CBU0698 to CBU0706) of the LPS encoding region (Fig. 2B) indicating deletions of these genes in a significant proportion of the cell population and a phase variation moving into a phase II form. The data also demonstrated that the occurrence of (growing) deletions is most likely an on-going event during passage since the read-mapping indicates a mixed metagenomic population at this LPS encoding region of the genome.

\section{Mouse bioassay}

All mice remained clinically healthy during the course of the experiment. However, at autopsy mice inoculated with $C$. burnetii strains showed significant splenomegaly as compared to control mice. The mice bioassay's showed a statistically significant level of splenomegaly in infected animals compared to the respective controls ( $p<0.01)$, except for the cell-based grown Scurry strain (E1) group $(p=0.10)$.

\section{Relative virulence of $C$. burnetii strains in mice}

The relative virulence of $C$. burnetii strains was expressed as the "relative spleen weight per number of C. burnetii bacteria" which considers both the degree of splenomegaly and the number of C. burnetii present in the spleen of infected mice. The in vivo growth of bacteria in the mice spleens were measured by qPCR assay, where the bacterial DNA was quantified by CBU_0407a single copy gene (results not shown). As shown in Fig. 3 and Table 1, the relative virulence of a particular strain is not significantly affected by the in vitro system that is used to propagate the bacteria $(p>0.01)$. These results indicate that the phenotypic expression of virulence of the used $C$. burnetii strains in mice is independent of the in vitro culture system chosen for propagating the strains. No statistically significant differences for the relative virulence $(p>0.01)$ were obtained between cell-based cultured strains of 3262 and 601 in these two independent experiments (E1 and E2), clearly highlighting the reproducibility for this measure in the mouse virulence bioassay. 


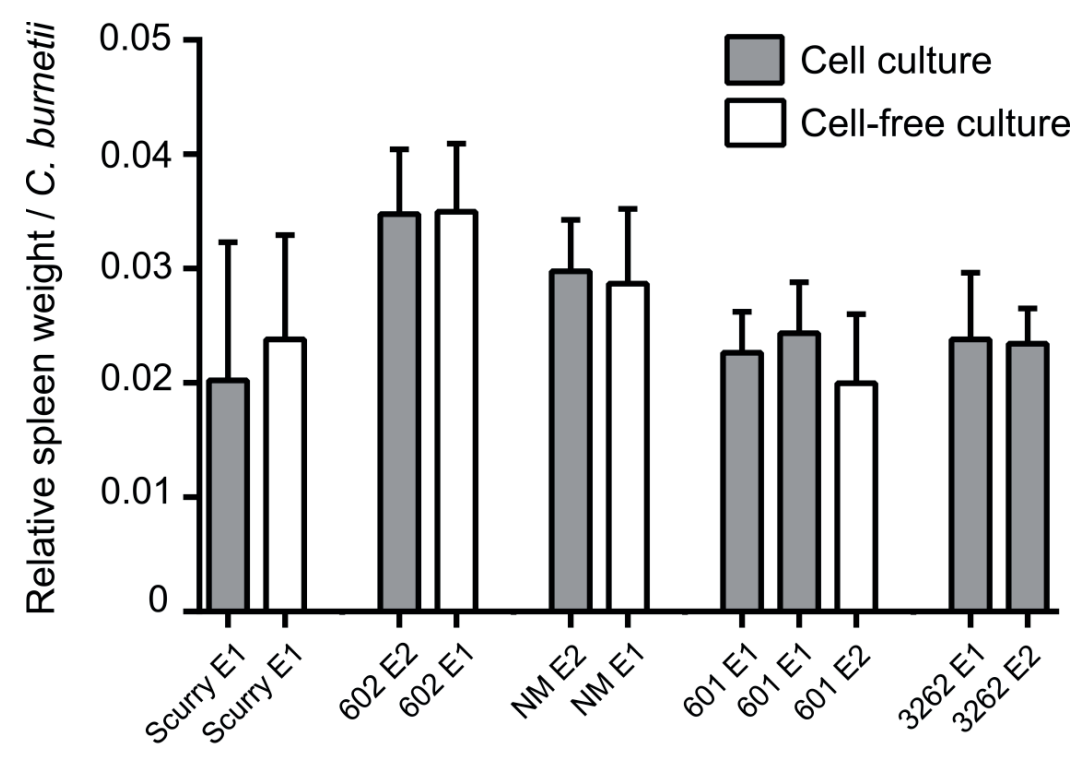

Strains

Figure 3. Changes in relative virulence. Relative virulence of different strains cultured in cell-based and cell-free systems expressed as the relative spleen weight per number of C. burnetii. E1, experiment 1; E2, experiment 2. 


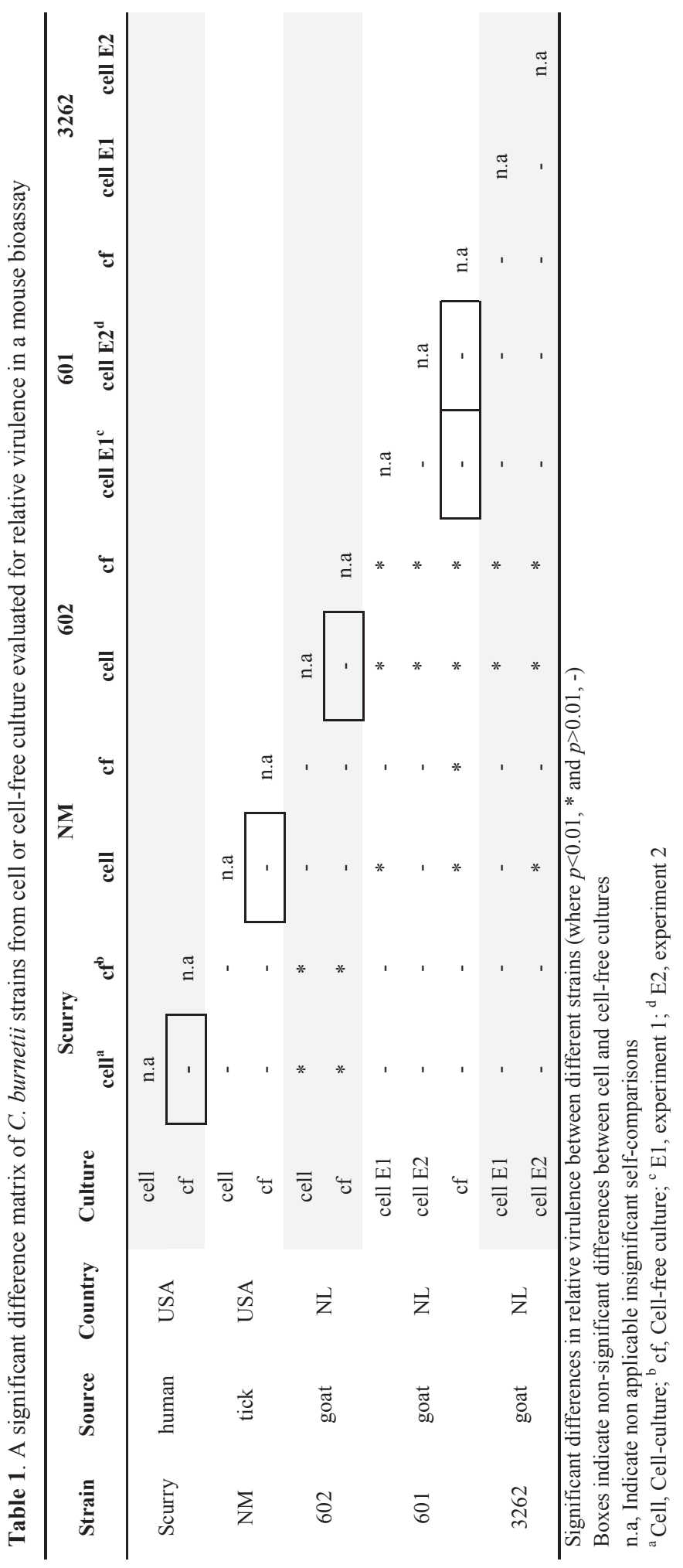




\section{Discussion}

In the present study we used three different methods to evaluate virulence-associated characteristics of different $C$. burnetii strains cultured in cell-based and cell-free systems. We did not observe any difference in the number of viable cells in the two culturing systems as determined by the use of PMAPCR. Furthermore, we did not find any evidence for the occurrence of loss of function of genes in the LPS encoding region in the low passaged strains from both the culturing systems. Since the loss of gene function of the genes in the LPS cluster can be associated with the variation of the virulent phase I to the avirulent phase II, we do not expect significant differences in the phase I to phase II ratios in the low passages of $C$. burnetii strains in both the culturing systems. Finally, we provide evidence that the relative virulence of $C$. burnetii strains is not affected by the cell-based or cell-free method of propagation as measured in an immune-competent mouse model. Therefore, our results clearly demonstrate that the cell-free culture system does not significantly influence the relative virulent phenotype of the tested strains. This indicates that the cell-free culturing system is invaluable for the identification and characterization of virulence factors of $C$. burnetii while allowing the investigation of molecular mechanisms involved in the pathogenesis of this obligate intracellular bacterium.

Our study reports the use of PMA-PCR, which worked better in our hands compared with EMA-PCR for quantification of viable cells of C. burnetii. The efficient quantification of genomic DNA from live cells by PMA or EMA treatment followed by PCR has been reported previously in different bacterial species [25,35,37-39]. It is one of the few current methods available for C. burnetii [25], which can be used to determine and quantify viable cells. Compared to PMA-PCR, decrease in PCR amplification was seen in EMA-PCR resulting in very low numbers of viable cells. In previous studies, EMA treatment showed loss of more than $60 \%$ genomic DNA due to penetration in live cells, whereas PMA was found to selectively penetrate only dead cells $[35,37,39,40]$. This higher impermeability of PMA through the intact cells might be due to the higher charge of PMA as suggested previously [35]. In samples treated at high temperatures, a high decrease in PCR amplification efficiency was seen compared to untreated cells, consistent with previously observations in other bacterial species. Therefore, a complete inhibition of PCR amplification of DNA from dead cells could not be expected at the tested concentrations of $C$. burnetii cells $[39,41,42]$. One of the important parameters to consider in this method is the matrix of the culture system the bacteria were propagated in, as non-specific host DNA also contributes in the efficiency of this technique. EMAPCR seemed to work well in egg homogenate matrix of $C$. burnetii which contains very high amounts of host DNA as previously described [25], but not on cell-based culture matrix which contain relatively less host DNA or no host DNA as in cell-free culture matrix. Hence, PMA-PCR was seen to mitigate the drawbacks observed with EMA-PCR in cell-based and cell-free culture systems. Also the viability counts obtained by PMA-PCR corresponded closely with direct enumeration FFU assay and CFU assay (data not shown) showing it as an efficient method to enumerate viable C. burnetii. 
Transcriptomics studies of all LPS encoding genes located in a $38 \mathrm{~kb}$ region in the C. burnetii genome is one of the robust and easy ways to detect loss of function of all these genes, which basically results in phase variation. The measured gene expression profiles showed all the low passaged strains of $C$. burnetii to be in phase I. Several other molecular techniques can also be used to determine the $C$. burnetii phase variation including approaches such as determining LPS sugar composition and quantification [43-46], PCR's on all genes of LPS coding regions [19], microarray-based genome comparisons [47], reverse transcriptase-PCR for expression of genes [19] and genome sequencing $[18,19]$. These methods are rather elaborative and tedious. Our result of transcriptomic measurements of a representative low passaged strain shows similar levels of expression of all genes in the LPS encoding region, without any significant differences in both cell-based and cell-free culture system. However, the high passaged cell-free cultured strain, showed significant $(\mathrm{p}<0.01)$ lower expression of several genes (CBU0676 to CBU0697) in major part of this region, compared with the low passaged cell-based and cell-free culture systems. Further, significant $(\mathrm{p}<0.01)$ down regulation of these genes with a fold change of $<4$ confirms the possible loss of gene function, which can result in a transition from phase I to phase II phenotype of the bacterium. This significant decrease of transcripts could also be regulatory and/or a physical deletion event within the LPS genes. Although lower, the expression of these genes are still detected suggesting the presence of mixtures of phase I and II variants of the strain, where the observed remaining transcripts of these genes are most likely from bacteria still in phase I form. High expression of genes was seen in this strain towards the end of the region (CBU0698 to CBU0706) suggesting the possible remains of these regions in the phase II variants.

Whole genome sequencing was performed to investigate whether any deletion events result in the observed low expression/regulation of genes involved in LPS synthesis. Here we used the depth of coverage in whole genome sequence data. A significant decrease in coverage was observed in the genes which showed low expression (CBU0676 to CBU0697) indicative for a deletion. Some of the deleted genes are involved in O-antigen biosynthesis or other LPS biosynthetic steps (Fig. 2) and its deletion may result in severe impairment of LPS synthesis correlating with phase variation $[18,48]$. Such large deletions were also seen previously in other phase II strains such as $9 \mathrm{Mi} / \mathrm{Baca}$ (deletion from CBU0678 to CBU0694), 9Mi/II/C1 and 9Mi/II/C4 (deletion from CBU0678 to CBU0698) $[18,19]$. The coverage of the genes (CBU0698 to CBU0706) present at the end of the LPS encoding region were higher, corresponding to the observed normal expression of these genes. Hence, the lower and higher read depths of genes obtained from sequencing (Fig. 2B) corresponded to lower and higher expression of the same genes obtained from expression studies (Fig. 2A). Similar results were also seen from SDS-PAGE analysis, where whole cell lysates of low and high passaged 602 strain had a similar profile of protein fragments which reflected those of the phase 1 type (bands between 21-14 $\mathrm{kDa}$ ). In addition, the high passaged strain comparatively had a more prominent band at $10.7 \mathrm{kDa}$ which is characteristic of phase II type. Hence, both phase I and phase II characteristic profiles were observed in high passaged cell-free cultured strains (Fig. 4) [34]. We conclude that low passaged 
strains from both the culture systems were in a predominant phase I form, and high passaged cell-free cultured strain shows possible deletions resulting in loss of gene function in the beginning of this LPS encoding region which is progressing throughout the region resulting in a phase II variant of the strain. Hence, cell surface LPS of C. burnetii is not significantly changed in the cell-free culture system similar to cell-based culture system in its early passages. Eventually phase variation occurs due to multiple passages in cell-free culture system, due to loss of LPS side chain as suggested previously [49].

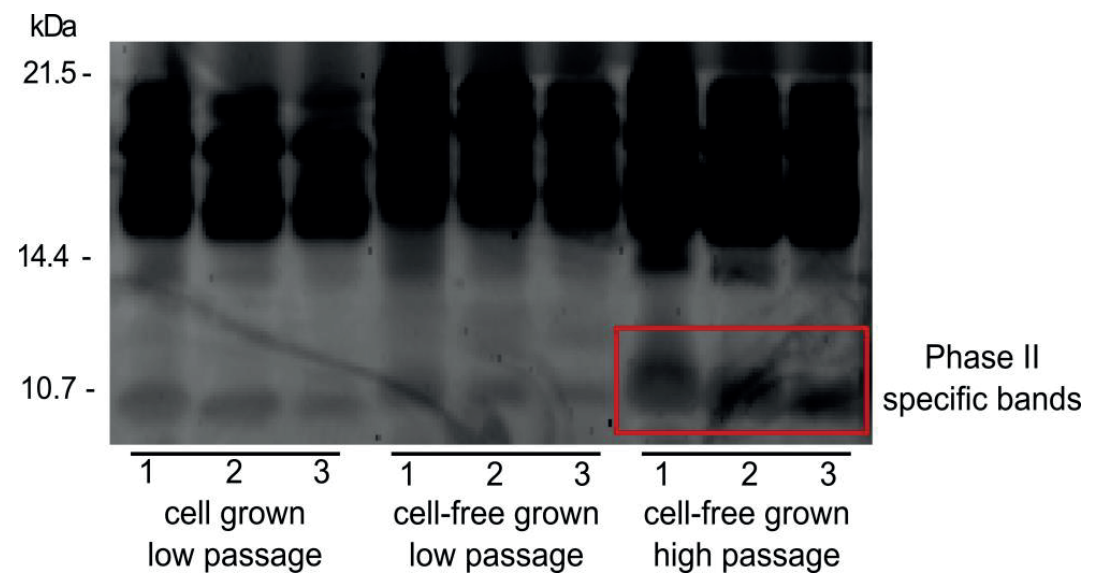

Figure 4. SDS-PAGE of C. burnetii whole cell lysates of low passaged cell-based and cell-free cultures and high passaged cell-free cultured 602 strain. Three serial dilutions (1:5) marked as 1,2 and 3 for all samples were used. The red box indicates the phase II specific bands seen in high passaged cell-free cultured strain.

In this present study we used immune-competent Swiss OF1 mice to evaluate the virulence properties of different $C$. burnetii strains. This model is currently used by several groups for evaluating pathogenicity of $C$. burnetii with consistent outcomes [28]. We assessed the virulence of $C$. burnetii strains using "splenomegaly" (increase in weight of spleen in relation to the body weight) and "RT-PCR quantification of bacteria in the spleen" as the most important read-out parameters $[13,15,50]$. Splenomegaly in response to infection was observed in these mice without any adverse clinical signs or mortality at 7 days post infection. Even at 21 days after infection no mortality of mice was observed and the splenomegaly reduced as well as the number of bacteria in the spleens, most likely indicating a level of clearance of the infection (data not shown). Also the degree of splenomegaly correlated with the bacterial load in the spleen as explained before [51]. All strains were capable of inducing pathological changes in the Swiss OF1 mice, showing its sensitivity to Q fever agent. 
The strains propagated in cell-based and cell-free culture systems did not show any significant differences in their relative virulence, showing relative virulence is independent of the culture system in which the strains are propagated in. We combined both, splenomegaly and bacterial load, which are most likely related, as virulence parameters to determine relative virulence of $C$. burnetii. The extent of splenomegaly and presence of $C$. burnetii in the spleen was strain dependent indicating that the strains most likely differentially affect the influx of (T) cells into the spleen and thereby facilitate bacterial clearance [15]. The colonization of spleen was a good indicator of infectivity, highlighting the relevance of these measurements for comparing strain virulence. Hence, the relative virulence showed strain-specific $(p<0.01)$ but similar virulence values $(p>0.01)$ for the same strain, irrespective the method of in vitro propagation for the tested low passage strains (Table 1). It is also expected that strains propagated from both the culture systems would equally loose virulence with respect to higher passages due to loss of LPS structure as seen previously [49]. Finally, the two individual experiments conducted showed reproducible relative virulence in 3262 and 601 cell-based cultured strains, underlining the precision of virulence associated measurements in the present mouse virulence bioassay.

In conclusion, our results provide evidence that the axenic culture system does not significantly influence the viability, phase variation and relative virulence of C. burnetii strains compared with cell-based culture. Thereby the cell-free propagation is an invaluable tool when studying molecular mechanisms underlying differences in virulence by using molecular genetic studies, which are not feasible using cell-based cultivation. Ultimately this system could be useful for development of effective subunit vaccines or production of recombinant antigens, which would offer great potential in the control of $\mathrm{Q}$ fever outbreaks as well as limiting its transmission to humans.

\section{Acknowledgements}

We thank the animal care takers, Annemieke Dinkla, Ruth Bossers-DeVries, Frank Harders and Anne Ammerdorffer for their technical support. 


\section{References}

1. Maurin M, Raoult D. Q fever. Clin. Microbiol. Rev. 1999;12:518-53.

2. Raoult D, Marrie T, Mege J. Natural history and pathophysiology of Q fever. Lancet Infect. Dis. 2005;5:219-26.

3. Angelakis E, Raoult D. Q fever. Vet. Microbiol. 2010;140:297-309.

4. Mazokopakis EE, Karefilakis CM, Starakis IK. Q fever endocarditis. Infect. Disord. Drug Targets. 2010;10:27-31.

5. Roest HIJ, Ruuls RC, Tilburg JJHC, Nabuurs-Franssen MH, Klaassen CHW, Vellema P, et al. Molecular epidemiology of Coxiella burnetii from ruminants in Q fever outbreak, the Netherlands. Emerg. Infect. Dis. 2011;17:668-75.

6. Roest HIJ, Tilburg JJHC, van der Hoek W, Vellema P, van Zijderveld FG, Klaassen CHW, et al. The Q fever epidemic in The Netherlands: history, onset, response and reflection. Epidemiol. Infect. 2011;139:1-12.

7. Kampschreur LM, Hagenaars JCJP, Wielders CCH, Elsman P, Lestrade PJ, Koning OHJ, et al. Screening for Coxiella burnetii seroprevalence in chronic Q fever high-risk groups reveals the magnitude of the Dutch Q fever outbreak. Epidemiol. Infect. 2013;141:847-51.

8. Enserink M. Questions Abound in Q-Fever Explosion in the Netherlands. Science. 2010;327:266-7.

9. van der Hoek W, Dijkstra F, Schimmer B, Schneeberger PM, Vellema P, Wijkmans C, et al. Q fever in the Netherlands: an update on the epidemiology and control measures. Euro Surveill. 2010;15.

10. Omsland A, Beare PA, Hill J, Cockrell DC, Howe D, Hansen B, et al. Isolation from animal tissue and genetic transformation of Coxiella burnetii are facilitated by an improved axenic growth medium. Appl. Environ. Microbiol. 2011;77:3720-5.

11. Omsland A, Cockrell DC, Howe D, Fischer ER, Virtaneva K, Sturdevant DE, et al. Host cell-free growth of the Q fever bacterium Coxiella burnetii. Proc. Natl. Acad. Sci. 2009;106:4430-4.

12. Stein A, Louveau C, Lepidi H, Ricci F, Baylac P, Davoust B, et al. Q Fever Pneumonia: Virulence of Coxiella burnetii Pathovars in a Murine Model of Aerosol Infection. Infect. Immun. 2005;73:246977.

13. Russell-Lodrigue KE, Andoh M, Poels MWJ, Shive HR, Weeks BR, Zhang GQ, et al. Coxiella burnetii isolates cause genogroup-specific virulence in mouse and guinea pig models of acute $\mathrm{Q}$ fever. Infect. Immun. 2009;77:5640-50.

14. Read AJ, Erickson S, Harmsen AG. Role of CD4+ and CD8+ T Cells in Clearance of Primary Pulmonary Infection with Coxiella burnetii. Infect. Immun. 2010;78:3019-26.

15. Andoh M, Zhang G, Russell-Lodrigue KE, Shive HR, Weeks BR, Samuel JE. T Cells Are Essential for Bacterial Clearance, and Gamma Interferon, Tumor Necrosis Factor Alpha, and B Cells Are Crucial for Disease Development in Coxiella burnetii Infection in Mice. Infect. Immun. 2007; 75:3245-55.

16. Moos A, Hackstadt T. Comparative virulence of intra- and interstrain lipopolysaccharide variants of Coxiella burnetii in the guinea pig model. Infect. Immun. 1987;55:1144-50. 
17. Stoker MG, Fiset P. Phase variation of the Nine Mile and other strains of Rickettsia burneti. Can. J. Microbiol. 1956;2:310-21.

18. Hoover TA, Culp DW, Vodkin MH, Williams JC, Thompson HA. Chromosomal DNA deletions explain phenotypic characteristics of two antigenic variants, phase II and RSA 514 (crazy), of the Coxiella burnetii nine mile strain. Infect. Immun. 2002;70:6726-33.

19. Denison AM, Massung RF, Thompson HA. Analysis of the O-antigen biosynthesis regions of phase II isolates of Coxiella burnetii. FEMS Microbiol. Lett. 2007;267:102-7.

20. Narasaki CT, Toman R. Lipopolysaccharide of Coxiella burnetii. Adv. Exp. Med. Biol. 2012;984:65-90.

21. Beare PA, Larson CL, Gilk SD, Heinzen RA. Two Systems for Targeted Gene Deletion in Coxiella burnetii. Appl. Environ. Microbiol. 2012;78:4580-9.

22. Beare PA, Gilk SD, Larson CL, Hill J, Stead CM, Omsland A, et al. Dot/Icm Type IVB Secretion System Requirements for Coxiella burnetii Growth in Human Macrophages. mBio. 2011;2:e00175-11.

23. Beare PA, Sandoz KM, Omsland A, Rockey DD, Heinzen RA. Advances in Genetic Manipulation of Obligate Intracellular Bacterial Pathogens. Front. Microbiol. [Internet]. 2011 [cited 2016 Nov 26];2. Available from: http://www.ncbi.nlm.nih.gov/pmc/articles/PMC3153054/

24. Beare PA, Howe D, Cockrell DC, Omsland A, Hansen B, Heinzen RA. Characterization of a Coxiella burnetii ftsZ mutant generated by Himar1 transposon mutagenesis. J. Bacteriol. 2009;191:1369-81.

25. Mori M, Boarbi S, Michel P, Bakinahe R, Rits K, Wattiau P, et al. In Vitro and In Vivo Infectious Potential of Coxiella burnetii: A Study on Belgian Livestock Isolates. PLoS ONE. 2013;8:e67622.

26. Brennan RE, Samuel JE. Evaluation of Coxiella burnetii antibiotic susceptibilities by real-time PCR assay. J. Clin. Microbiol. 2003;41:1869-74.

27. Howe D, Barrows LF, Lindstrom NM, Heinzen RA. Nitric Oxide Inhibits Coxiella burnetii Replication and Parasitophorous Vacuole Maturation. Infect. Immun. 2002;70:5140-7.

28. Rodolakis A. Q fever in France, International Q fever conference. Breda, Netherlands; 2010.

29. Buzoni-Gatel D, Rodolakis A. A mouse model to compare virulence of abortive and intestinal ovine strains of Chlamydia psittaci: influence of the route of inoculation. Ann. Microbiol. (Paris). 1983;134A:91-9.

30. Roest H-J, van Gelderen B, Dinkla A, Frangoulidis D, van Zijderveld F, Rebel J, et al. Q Fever in Pregnant Goats: Pathogenesis and Excretion of Coxiella burnetii. PLoS ONE. 2012;7:e48949.

31. Arricau Bouvery N, Souriau A, Lechopier P, Rodolakis A. Experimental Coxiella burnetii infection in pregnant goats: excretion routes. Vet. Res. 2003;34:423-33.

32. Tang F, Bossers A, Harders F, Lu C, Smith H. Comparative genomic analysis of twelve Streptococcus suis (pro)phages. Genomics. 2013;101:336-44.

33. Langmead B, Salzberg SL. Fast gapped-read alignment with Bowtie 2. Nat. Methods. 2012;9:3579 . 
34. Lukácová M, Kazár J, Gajdosová E, Vavreková M. Phase variation of lipopolysaccharide of Coxiella burnetii, strain Priscilla during chick embryo yolk sac passaging. FEMS Microbiol. Lett. 1993;113:285-9.

35. Nocker A, Cheung C-Y, Camper AK. Comparison of propidium monoazide with ethidium monoazide for differentiation of live vs. dead bacteria by selective removal of DNA from dead cells. J. Microbiol. Methods. 2006;67:310-20.

36. Leroy Q, Lebrigand K, Armougom F, Barbry P, Thiéry R, Raoult D. Coxiella burnetii Transcriptional Analysis Reveals Serendipity Clusters of Regulation in Intracellular Bacteria. PLoS ONE. 2010;5:e15321.

37. Nocker A, Camper AK. Selective Removal of DNA from Dead Cells of Mixed Bacterial Communities by Use of Ethidium Monoazide. Appl. Environ. Microbiol. 2006;72:1997-2004.

38. Nogva HK, Drømtorp SM, Nissen H, Rudi K. Ethidium monoazide for DNA-based differentiation of viable and dead bacteria by 5'-nuclease PCR. BioTechniques. 2003;34:804-8, 810, 812-3.

39. Pan Y, Breidt F. Enumeration of viable Listeria monocytogenes cells by real-time PCR with propidium monoazide and ethidium monoazide in the presence of dead cells. Appl. Environ. Microbiol. 2007;73:8028-31.

40. Yáñez MA, Nocker A, Soria-Soria E, Múrtula R, Martínez L, Catalán V. Quantification of viable Legionella pneumophila cells using propidium monoazide combined with quantitative PCR. J. Microbiol. Methods. 2011;85:124-30.

41. Wagner AO, Malin C, Knapp BA, Illmer P. Removal of free extracellular DNA from environmental samples by ethidium monoazide and propidium monoazide. Appl. Environ. Microbiol. 2008;74:2537-9.

42. Cawthorn D-M, Witthuhn RC. Selective PCR detection of viable Enterobacter sakazakii cells utilizing propidium monoazide or ethidium bromide monoazide. J. Appl. Microbiol. 2008;105:117885 .

43. Baca OG, Martinez IL, Aragón AS, Klassen D. Isolation and partial characterization of a lipopolysaccharide from phase II Coxiella burnetii. Can. J. Microbiol. 1980;26:819-26.

44. Schramek S, Mayer H. Different sugar compositions of lipopolysaccharides isolated from phase I and pure phase II cells of Coxiella burnetii. Infect. Immun. 1982;38:53-7.

45. Amano K, Williams JC, McCaul TF, Peacock MG. Biochemical and immunological properties of Coxiella burnetii cell wall and peptidoglycan-protein complex fractions. J. Bacteriol. 1984;160:982-8.

46. Mayer H, Radziejewska-Lebrecht J, Schramek S. Chemical and immunochemical studies on lipopolysaccharides of Coxiella burnetii phase I and phase II. Adv. Exp. Med. Biol. 1988;228:577-91.

47. Beare PA, Samuel JE, Howe D, Virtaneva K, Porcella SF, Heinzen RA. Genetic Diversity of the Q Fever Agent, Coxiella burnetii, Assessed by Microarray-Based Whole-Genome Comparisons. J. Bacteriol. 2006;188:2309-24.

48. Seshadri R, Paulsen IT, Eisen JA, Read TD, Nelson KE, Nelson WC, et al. Complete genome sequence of the Q-fever pathogen Coxiella burnetii. Proc. Natl. Acad. Sci. U. S. A. 2003;100:545560. 
49. Kersh GJ, Oliver LD, Self JS, Fitzpatrick KA, Massung RF. Virulence of pathogenic Coxiella burnetii strains after growth in the absence of host cells. Vector Borne Zoonotic Dis. Larchmt. N. 2011;11:1433-8.

50. Kazár J, Lesý M, Propper P, Valková D, Brezina R. Comparison of virulence for guinea pigs and mice of different Coxiella burnetii phase I strains. Acta Virol. 1993;37:437-48.

51. Zhang GQ, Samuel JE. Identification and cloning potentially protective antigens of Coxiella burnetii using sera from mice experimentally infected with Nine Mile phase I. Ann. N. Y. Acad. Sci. 2003;990:510-20. 



\section{Chapter $\mathbf{3}$}

\section{Major differential gene regulation in Coxiella burnetii between in vivo and}

in vitro cultivation models

Runa Kuley, Ruth Bossers de-Vries, Hilde E. Smith, Mari A. Smits, Hendrik I.

J. Roest and Alex Bossers

BMC Genomics. 2015 Nov 16;16:953 


\begin{abstract}
Background

Coxiella burnetii is the causative agent of the zoonotic disease Q fever. As it is an intracellular pathogen, infection by $C$. burnetii requires adaptation to its eukaryotic host and intracellular environment. The recently developed cell-free medium also allows the bacteria to propagate without host cells, maintaining its infection potential. The adaptation to different hosts or extracellular environments has been assumed to involve genome-wide modulation of $C$. burnetii gene expression. However, little is currently known about these adaptation events which are critical for understanding the intracellular survival of $C$. burnetii.
\end{abstract}

\title{
Results
}

We studied C. burnetii genome-wide transcriptional patterns in vivo (mice spleen) and in cell and cellfree in vitro culture models to examine its metabolic pathways and virulence associated gene expression patterns that are required to colonize and persist in different environments. Within each model, the gene expression profiles of the Dutch C. burnetii outbreak strain (602) and NM reference strains were largely similar. In contrast, modulation of gene-expression was strongly influenced by the cultivation method, indicating adaptation of the bacterium to available components. Genome-wide expression profiles of C. burnetii from in vitro cell culture were more similar to those seen for in vivo conditions, while gene expression profiles of cell-free culture were more distant to in vivo. Under in vivo conditions, significant alterations of genes involved in metabolism and virulence were identified. We observed that $C$. burnetii under in vivo conditions predominantly uses glucose as a carbon source (mostly for biosynthetic processes) and fatty acids for energy generation. C. burnetii experienced nutrient limitation and anaerobiosis as major stressors, while phosphate limitation was identified as an important signal for intracellular growth inside eukaryotic host cells. Finally, the in vivo environment significantly induced expression of several virulence genes, including those implicated in LPS synthesis, colonization, host component modulation and DNA repair mechanisms.

\section{Conclusion}

Our study shows that $C$. burnetii, with its relative small genome, requires only a subset of core gene functions to survive under in vitro conditions, but requires the induction of full repertoire of genes for successful pathogenesis and thriving in harsh environments in vivo. 


\section{Background}

Q fever, a worldwide zoonotic infectious disease, is caused by Coxiella burnetii, an intracellular Gram negative bacterium. Domestic ruminants (e.g., goat, sheep and cattle) are considered the main reservoir for $\mathrm{Q}$ fever infections in humans; the bacterium can cause a range of diseases depending on the host [1-3]. The main clinical manifestation of $\mathrm{Q}$ fever in goats and sheep are abortions, which result in the shedding of large numbers of bacteria into the environment. Inhalation of such contaminated aerosols is the main route of transmission in humans and can lead to acute or chronic Q fever [1, 4]. Acute infections range from asymptomatic to abrupt flu-like illness or pneumonia [5]; chronic infection is typically manifested as endocarditis [6]. An unprecedented outbreak of Q fever occurred in the Netherlands during the years 2007-2010, with more than 4,000 human cases registered; infected dairy goats and sheep were identified as the primary source of the disease [7-11] .

Attempts to understand the biology and pathogenesis of this bacterium through molecular techniques have been hampered by its intracellular lifestyle. However, a better understanding of the interactions of the pathogen with the host could be gained using global transcription profiles. This might aid in determining adaptations of the bacterium within the infected host and provide a fuller appreciation of its pathogenicity.

As an obligate intracellular pathogen, C. burnetii resides and proliferates within the acidic parasitophorous vacuole (PV) of the host cells [12]. The bacteria can infect a wide range of hosts and can also propagate to high quantities in different in vitro culture models [1, 13-15]. Within a laboratory setup, immune-competent mice are usually used as the in vivo animal model to assess the infection potential of C. burnetii [16]. The virulence of the strains is often measured based on splenomegaly and load of the bacteria in the spleen of mice [17-19]. C. burnetii are routinely cultured in BGM cells [15, 20], Vero cells [21] or macrophage like cell lines [12, 22]. The bacterium can establish persistent infections in cell culture, where it multiplies and survives in the cell PV. The latest advancement in Q fever research is the development of a host cell-free medium, designed based on the metabolic requirements of the bacterium. This complex nutrient medium supports substantial growth of most $C$. burnetii isolates and overcomes the drawbacks encountered when studying the pathogen in cell based culture or in vivo models [14, 15, 23].

Availability of complete genome sequences of $C$. burnetii contributed significantly towards understanding of the physiology and the pathogenic abilities of the bacterium [24]. However, information regarding environmental adaptations and expression of potential virulence factors important for bacterial colonization, replication and persistence under in vivo and in vitro systems is not entirely clear. Such information will provide insights into processes occurring during intracellular and extracellular growth of $C$. burnetii and allow direct evaluation of the relationship between gene expression patterns during growth inside living hosts and under in vitro conditions.

In this study, we endeavor to understand how the pathogen adapts to the host microenvironment, by monitoring the expression changes across the whole transcriptome of $C$. 
burnetii obtained within different propagation models. Main reservoirs of Q fever, such as pregnant goat and sheep, harbor the pathogen primarily in placentas, making this an ideal in vivo sample [20]. The bacterium has also been detected in oviducts and genital tissues of naturally infected non-pregnant goats [25]. However, use of these models to assess in vivo C. burnetii global transcriptional profiles is hampered by technical difficulties regarding the required fresh aborted sample collection (placentas), low RNA concentrations and quality. To avoid these limitations we used the spleens of experimentally infected mice as an in vivo model. We report here the global transcriptional pattern of $C$. burnetii from this in vivo model (mice spleen) compared with C. burnetii grown under laboratory conditions using cell-based and cell-free culture methods. In addition, we allowed strains to adapt from cell to cell-free culture propagation and considered the first two passages to determine the adaptation capabilities of the bacterium as it moves from one environment (propagation inside host cells) to another (cell-free cultivation). Our study provides a comprehensive view of $C$. burnetii metabolic adaptations and other important process required for its survival in response to diverse environments.

\section{Results and discussion}

Preparation of $C$. burnetii cells from different propagation models and measurement of its gene expression patterns

A whole-genome microarray was constructed based on all the available annotated genome sequences of $C$. burnetii in the databases. The mRNA levels of the expressed genes were assessed under in vivo and various in vitro conditions. For the in vivo transcriptome analysis, C. burnetii obtained from infected mice spleens were chosen, as spleens are highly responsive to infections and bacterial colonization in spleen is a good indicator of infectivity and persistence over time [17-19,26]. During the time of sampling, all infected mice showed splenomegaly and an almost 10 fold increase in number of bacteria in the spleen. This spleen mice model offers several benefits, although it also has limitations, such as the usage of intraperitoneal (IP) infection route rather than intranasal route which is the best natural exposure model. However, because no significant changes in splenomegaly or bacterial numbers in spleens were observed between different inoculation routes, the IP route seems an easy and efficient inoculation route for C. burnetii infections in mice [27]. We determined the global transcriptional patterns of $C$. burnetii at 20 days p.i., because host immune responses towards bacterial infection were high around this period (data not shown) allowing us to examine bacterial metabolic adaptations, role of virulence factors and mechanisms to evade host defenses. C. burnetii infection in mice usually results in higher bacterial numbers in spleen at early infection stages (7-14 days) followed by significant decrease in bacteria around 20 days. This most likely indicates an influx of immune (T) cells into the spleen around 7 days p.i., resulting in increased splenomegaly and facilitating bacterial clearance thereafter [17]. To obtain sufficient bacterial RNA from spleens at day 20 , a higher dose of bacteria $\left(10^{7}\right.$ genome equivalents) were inoculated in our mice. This high dosage was checked in our previous study, which showed that for mice given this dosage, splenomegaly and 
bacterial replication rate patterns at different time periods (Fig S1) were similar to a lower dosage $\left(10^{4}\right.$ genome equivalents) [26]. Transcriptional profiles of C. burnetii from later stage of infection (20 days), where the bacterial numbers are significantly lower than at early infection stages, were compared with stationary phases of in vitro grown bacterium (cell and cell-free cultures) to examine environmentally-linked gene expression differences of the bacterium. To further study the various adaptations of the bacterium, C. burnetii propagated from first two serial passages in cell-free culture (p1 and p2) from cell-cultured strains and (fully) adapted cell-free cultured strains from stationary phase were considered. Gene expression of all cell-free cultured strains (p1, p2 and adapted) was compared with gene expression from cell culture as reference.

\section{Impact of cultivation conditions on $C$. burnetii transcriptome}

Good quality RNA was isolated from $C$. burnetii originating from different culture systems by depletion of the host RNA (except in cell-free culture system). The RNA was successfully labelled and used for microarray analysis (in triplicate). Principal component analysis (PCA) plots were constructed, which clearly showed that most sample triplicate clustered tightly, indicative of good experimental reproducibility of samples for each strain in different culture systems (Fig 1).

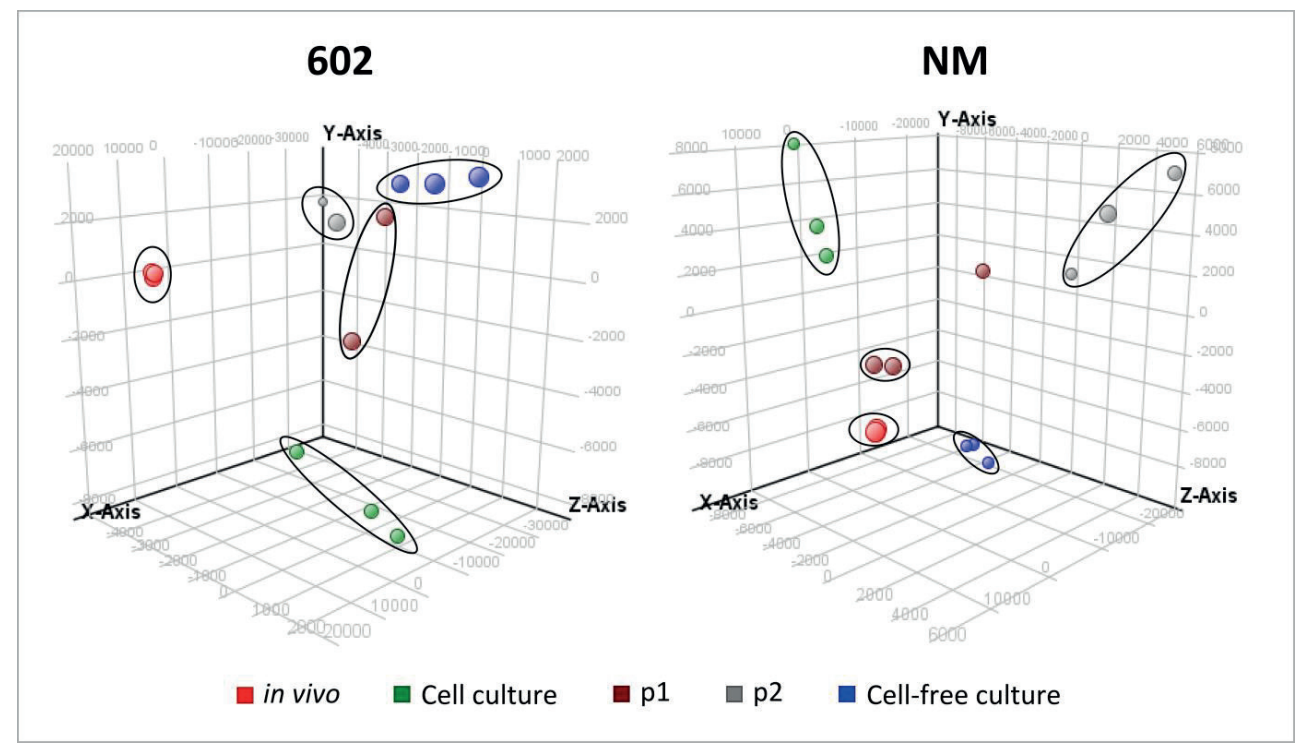

Figure 1. Principal component analysis on genome-wide expression profiles of C. burnetii strains 602 and NM obtained from various culture systems. Triplicate findings for each sample from different culture systems are clustered together indicative for experimental reproducibility. The plots of independent samples demonstrate also that based on their transcriptome profile, samples from different culture models can be differentiated. 


\section{Niche specific gene expression patterns of $C$. burnetii}

To get an overview of the gene expression of 602 and NM strains induced by different culture models, hierarchical clustering was performed based on average normalized signal intensity of probes. Clustering showed that the expression profiles of strains are largely determined by the method in which they were cultured or isolated (Fig. 2). The expression profiles of C. burnetii isolated from in vivo and in vitro models clustered separately. Within the in vitro culture models, expression profiles of strains from cell culture were closer to the in vivo model, followed by expression profiles of strains obtained from the cell-free culture model. These results suggest that gene expression of C. burnetii depends on the available components present in the growth medium, tissue or cell. Hence, the possible differences in the metabolic capabilities of the strains are clearly seen in culture models considered in the study.

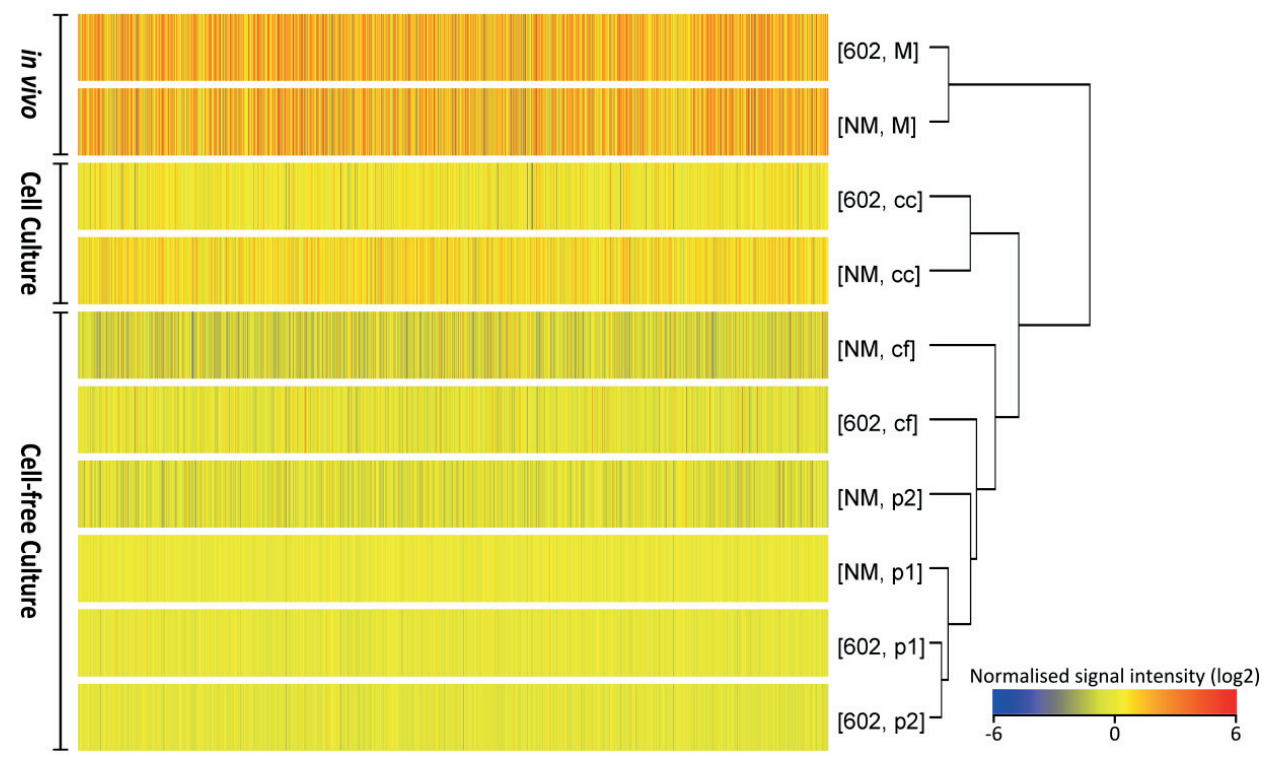

Figure 2. Hierarchical clustering analysis on normalized signal intensities of probes of 602 and NM strains in different culture models. Data shows clustering of in vivo and in vitro models separately based on patterns of gene expression. Normalized signal intensity $(\log 2)$ of probes (average of triplicates) for each condition are represented as a color scale from red for high expressions to blue for lower expressions. In vivo $(\mathrm{M}=$ mice spleen), cell culture (cc), cell-free adapting phase passage 1 (p1) and passage 2 (p2), cell-free culture model (cf).

\section{The global transcription profile in different culture models}

Because the different strains have transcriptional profile clustering based on the culture model in which it was propagated, thorough studies of gene expression were conducted. We determined which genes showed major differential expression in the spleens of mice compared to in vitro models. 
Moreover, we looked into differences in gene expression that existed between in vitro culture models. These detailed studies were conducted using the data from the Dutch outbreak strain 602, which was shown to be highly virulent by our previous mouse virulence bioassays [15]. GeneSpring GX microarray software analysis showed 906 of the 2061 genes were, compared to in vitro cell culture, differentially regulated ( $p<0.05$, fold change of 2 ) in 602 strains from mice spleen, with a majority of genes (67\%) up-regulated. Comparing expression profiles of the 602 strain under in vitro conditions (cell-free vs. cell culture model) showed that 438 genes were differentially regulated, but the majority

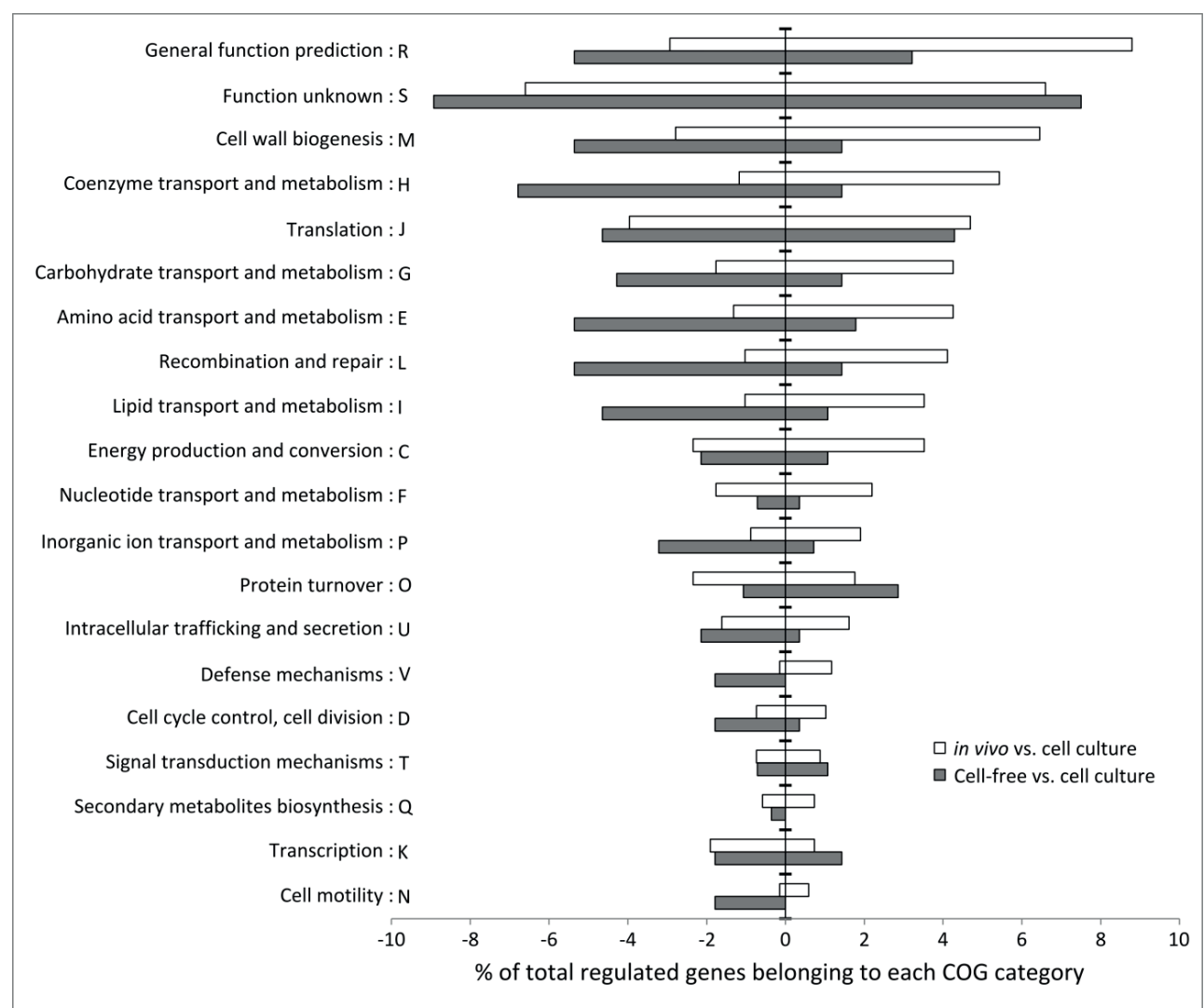

Figure 3. Functional COG-categories of differentially regulated C. burnetii genes under in vivo and in vitro culture models. Functional categories of regulated genes of the 602 strain under in vivo and cell-free culture compared with cell culture model. The up-regulated and down-regulated genes are shown on the right and left side of the $y$-axis respectively. Largest group of regulated genes in all culture models belonged to the unknown function category (S). Large number of up-regulated genes under in vivo conditions belonged to the metabolism group such as coenzyme, carbohydrate, amino acid and lipid transport and metabolism (HGEI). Whereas, the largest number of up-regulated genes in cell-free culture compared to cell culture belonged to the protein synthesis group $(\mathrm{O})$. 
Of genes (71\%) were down-regulated. A complete microarray dataset from the different comparisons are presented as supplementary material (Table S1).

Table 1. Significantly regulated (KEGG) Pathways in different culture models of $C$. burnetii

\begin{tabular}{|c|c|c|c|c|c|}
\hline Pathway ID & Pathway & m vs. cc & p1 vs ce & p2 vs. ce & cf vs ce \\
\hline & Metabolic pathways & & & & \\
\hline cbu00230 & Purine metabolism & $5.20 \mathrm{E}-18^{*}$ & $2.00 \mathrm{E}-01$ & $5.40 \mathrm{E}-06^{*}$ & $1.10 \mathrm{E}-12 *$ \\
\hline cbu00010 & Glycolysis/ Gluconeogenesis & $1.00 \mathrm{E}-13 *$ & $3.80 \mathrm{E}-03 *$ & $1.50 \mathrm{E}-07 *$ & $1.50 \mathrm{E}-07 *$ \\
\hline cbu00190 & Oxidative phosphorylation & $3.10 \mathrm{E}-10 *$ & $2.40 \mathrm{E}-01$ & $1.90 \mathrm{E}-02 *$ & $1.30 \mathrm{E}-01$ \\
\hline cbu00240 & Pyrimidine metabolism & $5.20 \mathrm{E}-06^{*}$ & $9.80 \mathrm{E}-03 *$ & $1.80 \mathrm{E}-08^{*}$ & $5.60 \mathrm{E}-05^{*}$ \\
\hline cbu00564 & Glycerophospholipid metabolism & $1.80 \mathrm{E}-05^{*}$ & $6.30 \mathrm{E}-02$ & $6.00 \mathrm{E}-02$ & $6.80 \mathrm{E}-02$ \\
\hline cbu00020 & Citrate cycle (TCA cycle) & $9.20 \mathrm{E}-04 *$ & - & $3.40 \mathrm{E}-02 *$ & $5.30 \mathrm{E}-02$ \\
\hline cbu00071 & Fatty acid metabolism & $3.10 \mathrm{E}-03 *$ & - & - & - \\
\hline cbu00061 & Fatty acid biosynthesis & $3.20 \mathrm{E}-03 *$ & $1.40 \mathrm{E}-02 *$ & $7.40 \mathrm{E}-02$ & $7.90 \mathrm{E}-02$ \\
\hline
\end{tabular}

Biosynthesis of secondary metabolites

$\begin{array}{lllccc}\text { cbu00900 } & \text { Terpenoid backbone biosynthesis } & 2.80 \mathrm{E}-05^{*} & - & - & - \\ \text { cbu00780 } & \text { Biotin metabolism } & 1.00 \mathrm{E}-04 * & 1.00 \mathrm{E}-03 * & 2.30 \mathrm{E}-03 * & 2.40 \mathrm{E}-06 * \\ \text { cbu00730 } & \text { Thiamine metabolism } & 6.10 \mathrm{E}-04 * & 7.90 \mathrm{E}-03 * & 9.80 \mathrm{E}-03 * & 1.30 \mathrm{E}-05 *\end{array}$

\section{Biosynthesis of amino acids}

$\begin{array}{lllllc}\text { cbu00400 } & \text { Aromatic amino acids biosynthesis } & 8.40 \mathrm{E}-08^{*} & - & - & - \\ \text { cbu00250 } & \text { Alanine, aspartate and glutamate metabolism } & 3.40 \mathrm{E}-05^{*} & - & 2.30 \mathrm{E}-02 * & 1.20 \mathrm{E}-03 * \\ \text { cbu00260 } & \text { Glycine, serine and threonine metabolism } & 2.20 \mathrm{E}-04 * & 1.60 \mathrm{E}-04 * & 2.30 \mathrm{E}-06 * & 1.10 \mathrm{E}-04 *\end{array}$

\section{Virulence}

$\begin{array}{llcccc}\text { cbu00540 } & \text { Lipopolysaccharide biosynthesis } & 5.40 \mathrm{E}-10^{*} & 2.80 \mathrm{E}-04 * & 3.70 \mathrm{E}-05^{*} & 1.90 \mathrm{E}-04 * \\ \text { cbu03070 } & \text { Bacterial secretion system } & 7.40 \mathrm{E}-08^{*} & 6.90 \mathrm{E}-02 & - & 1.30 \mathrm{E}-03 *\end{array}$

\section{Repair mechanisms}

$\begin{array}{llllcl}\text { cbu03430 } & \text { Mismatch repair } & 7.90 \mathrm{E}-13^{*} & - & 3.60 \mathrm{E}-06^{*} & 1.50 \mathrm{E}-17 * \\ \text { cbu03410 } & \text { Base excision repair } & 1.00 \mathrm{E}-07^{*} & - & 3.40 \mathrm{E}-02 * & 2.50 \mathrm{E}-05^{*} \\ \text { cbu03420 } & \text { Nucleotide excision repair } & 8.00 \mathrm{E}-07^{*} & - & - & 4.50 \mathrm{E}-02 *\end{array}$

\section{Others}

\begin{tabular}{|c|c|c|c|c|c|}
\hline cbu00550 & Peptidoglycan biosynthesis & $4.00 \mathrm{E}-13 *$ & - & - & $3.30 \mathrm{E}-03 *$ \\
\hline cbu02010 & $\mathrm{ABC}$ transporters & $1.00 \mathrm{E}-04 *$ & - & $4.00 \mathrm{E}-02 *$ & $1.10 \mathrm{E}-03 *$ \\
\hline cbu02020 & Two-component system & $3.50 \mathrm{E}-03 *$ & - & - & - \\
\hline
\end{tabular}

The $P$ value (adjusted by Benjamini-Hochberg method) of regulated pathway is shown for each comparison of 602 strain. Significant pathways $(p<0.05)$ are depicted with *. In vivo $(\mathrm{m}=$ Mice spleen $)$, cell culture $(\mathrm{cc})$, cellfree adapting phase passage 1 (p1), cell-free adapting phase passage 2 (p2), cell-free culture model (cf) 
Next, in various growth models, we determined the functional categories [28] and performed pathway enrichment analysis of the differentially regulated genes (Fig 3 and Table 1). In the in vivo model, when compared with genes expressed in cell culture, with the exception of the group of unknown, hypothetical and conserved hypothetical proteins (44\% of in vivo induced genes), the largest group of functional category genes (HEGCIFPQ) and pathways showing increased expression belonged to the metabolism group (Fig 3, Table 1). In contrast, fewer genes and pathways involved in metabolism were up-regulated in the cell-free culture model than in the cell culture model. Apart from the metabolic genes, other genes seen to be highly up-regulated in vivo when compared to cell culture belonged to defense mechanisms (V), cell wall biogenesis $(\mathrm{M})$ and recombination and repair $(\mathrm{L})$ categories probably due to bacterial resistance to the harsh environments of PV. In cell-free culture $16 \%$ of induced genes belonged to translation $(\mathrm{J})$ and post translational modifications/protein-turnover (O) categories indicating more protein synthesis leading to faster growth rates compared to other conditions.

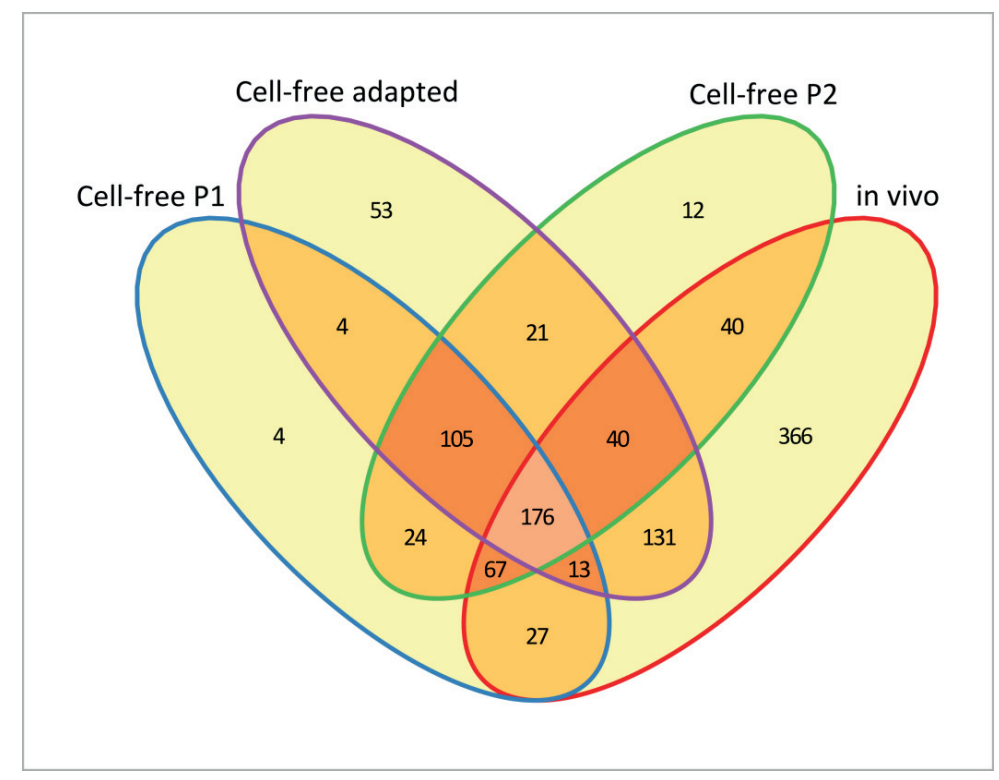

Figure 4. Venn diagram of differentially expressed genes of 602 strain in different culture models. Comparison of differentially regulated genes under in vivo, cell-free adapting serial passages (p1 and p2) and fully adapted culture with respect to in vitro growth in cell culture.

To gain insight into the distribution and uniqueness of regulated genes in the different culture models, Venn-diagrams were constructed (Fig 4). Comparison of differentially regulated genes under in vivo conditions and cell-free culture (including p1, p2 and cell-free adapted) with respect to cell culture showed 176 genes which were similarly regulated between different models and a large 
number of genes (366 genes) which were specifically regulated in vivo (Fig 4). These data suggest that gene expression of $C$. burnetii under in vivo conditions is quite different from its in vitro culture models.

Among the in vitro models, comparison between $\mathrm{p} 1$ and $\mathrm{p} 2$ cultures resulted in differential regulation of $0.6 \%$ of genes only, suggesting that these cultures were highly similar. Comparison of $\mathrm{p} 1$ and $\mathrm{p} 2$ cultures with cell-free adapted and cell culture showed differential regulation of $15 \%$ and $30 \%$ of genes respectively. This indicates that based on the transcriptomic profiles, $\mathrm{p} 1$ and $\mathrm{p} 2$ cultures are closer to cell-free adapted culture than those from cell culture. The $15 \%$ of genes that were differentially regulated in $\mathrm{p} 1$ and $\mathrm{p} 2$ compared to cell-free culture were further categorized into functional groups. About $40 \%$ of these differentially regulated genes in $\mathrm{p} 1$ and $30 \%$ in p2 were involved in metabolism categories (CEFGHIPQ), showing almost a $10 \%$ decrease in metabolism related genes in $\mathrm{p} 2$ within 1 passage, itself in a cell-free environment. This shows that $\mathrm{p} 1$ and $\mathrm{p} 2$ cultures regulate the genes in a way similar to adapted cell-free culture, most likely due to available resources in the culture media. Thus, the rapid adaptation capabilities of $C$. burnetii were seen when moved from one environment to another.

\section{C. burnetii requires metabolic adaptations for different culture models}

Striking differences were found between in vivo and in vitro culture models in metabolic pathway regulation. These differences could be attributed to the type of nutrient the bacteria were able to use for metabolism. Under in vivo conditions, genes implicated in glucose transportation (CBU0265), phosphorylation and glycolytic intermediates generation were up-regulated compared to in vitro models [29]. Further, the terminal enzymes of the glycolytic pathway were repressed and genes implicated in phospholipid synthesis were induced, indicating the use of glycolytic intermediates for phospholipid synthesis (Fig 5A). Among the different phospholipids [30], gene encoding cardiolipins $(\mathrm{cls})$, an anionic phospholipid were highly induced in vivo compared to in vitro conditions, indicating an increased synthesis of cardiolipins allowing the bacterium to survive in the highly acidic environment of spleen cell PV [31]. A low level expression of cls in cell and cell-free cultures might be sufficient for the bacterium to sustain under in vitro environments, while up-regulation in vivo is possibly required to cope with specific in vivo conditions associated with the acidic environment. In a previous study, cardiolipids were not identified by biochemical analysis of phospholipids in $C$. burnetii grown from in vitro embryonated eggs [32], implying its greater importance under in vivo conditions. These data confirm previous research indicating that the phospholipid content of microorganisms can differ based on the bacterial environment in order to cope with new environmental conditions [33].

Genes implicated in the synthesis of lipids were enhanced in vivo more than under in vitro conditions. Enhanced expression of a putative long-chain fatty acids translocation gene (CBU1242) across the outer membrane of the bacterium was seen, indicating its abundant presence in the PV of 
host spleen cells. Interestingly, genes coding de novo synthesis of fatty acid were also induced, probably to synthesize more fatty acids to meet the overall requirements of the bacterium. The data shows $C$. burnetii can acquire lipids through both de novo bacterial synthesis as well as by subversion of host cell pools as indicated previously [30]. Further, the mevalonate pathway, which encodes an isoprenoid backbone (Fig 5B), [24, 34] and other genes (uppS, ispB) that use isoprenoids as substrates were up-regulated in vivo and might help C. burnetii in cell wall synthesis and normal growth in host cells $[35,36]$. Apart from synthesis of its own lipids, it has been speculated that $C$. burnetii can use host cell cholesterol precursors and convert these into cholesterol. The genome of $C$. burnetii encodes putative $\Delta 24$ sterol reductase (CBU1206) the biological role of which is not known, but in general is involved in final stages of cholesterol synthesis in eukaryotic cells. Maintaining such a unique gene in its genome and its up-regulation suggests a critical function for this gene in the bacterium in vivo [3739]. The role of cholesterol in bacterial pathogenesis is unclear [40, 41], and cell culture of C. burnetii lacking cholesterol showed either no effects or negative effects on replication and PV morphology [38, 39]. Sterol reductase could be involved in cholesterol synthesis in limiting sterol conditions. However, since mammalian cells have high sterol content, the bacteria would not need to synthesize more cholesterol. It is more likely that up-regulation of sterol reductase is involved in generating novel sterol species that have signaling, structural roles or associated with oxidative stress responses [30, 40-43]. Within in vitro models, induction of these genes was seen in cell culture at lower levels than observed in the in vivo system, indicating that the synthesis of these lipids is only intracellular.

The size of the genome of $C$. burnetii is highly reduced (around $2 \mathrm{Mb}$ ) comparable to size in other obligate intracellular pathogens. This renders C. burnetii auxotrophic for several amino acids or causes it to lack key enzymes in several biosynthetic pathways [24]. Yet under in vivo conditions, several amino acid and oligopeptide transporters, pathways implicated in synthesis of aromatic amino acids (Fig 5C) $[24,44,45]$ and degradation of amino acids (L-serine, L-aspartate, L-glutamine, L-cysteine, L-methionine, L-proline) were highly induced. Taken together, these observations suggest that $C$. burnetii can acquire several amino acids from hosts and synthesizes a few that may be in limited supply due to severe nutritional stress in host cells. A lower level of nutritional stress can be inferred in in vitro conditions, since compared to in vivo only a few transporters are up-regulated in cell culture (CBU1798, CBU0539) and cell-free culture (CBU1130, artM).

A large number of genes coding several co-factors (biotin, thiamine, folic acid and Coenzyme A) was also up-regulated in vivo relative to in vitro culture models (Fig 5D,5E). The high level expression of these genes implicated in co-factor synthesis, might indicate lack of readily available cofactors in the spleens of mice. Contrary, it could be possible that the C. burnetii genome might not encode co-factor transporters and therefore synthesizes them since they are required for growth under in vivo conditions. The cell and cell-free culture media are rich in co-factors and down-regulation of pathways involved in co-factor synthesis; imply transportation of co-factors by the bacterium. As of now, specific co-factor transporters for these organic co-factors are not reported, but C. burnetii 


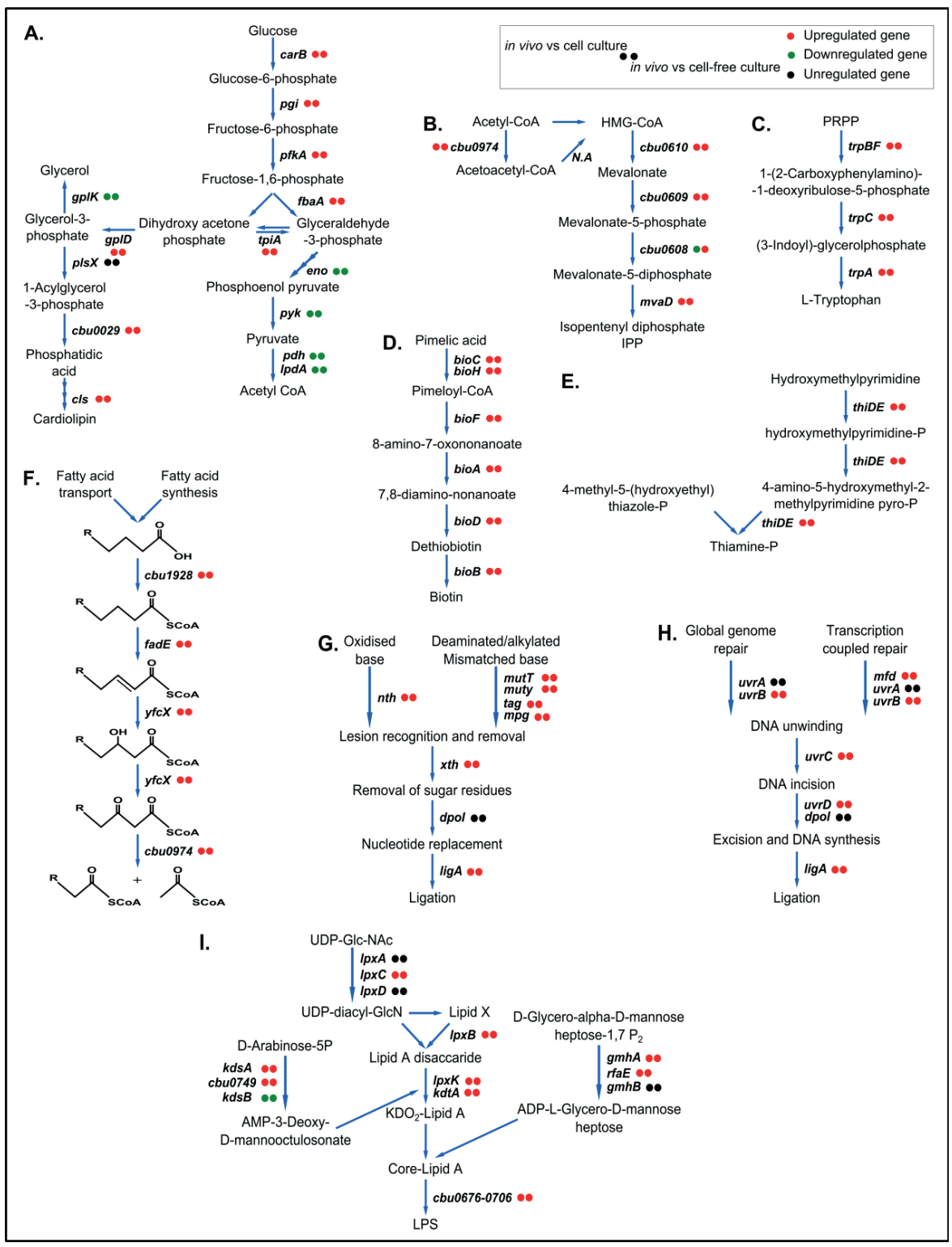

Figure 5. Some metabolic pathways and pathways implicated in virulence and repair mechanisms induced in vivo compared to in vitro cultivation. The pathways were drawn according to KEGG database. Circles next to each gene display its regulation for each condition. A-I represent significantly up-regulated KEGG pathways under in vivo conditions: A) Cardiolipin synthesis, B) Mevalonate pathway, C) Tryptophan synthesis, D) Biotin synthesis, E) Thiamine synthesis, F) Fatty acid degradation, G) Base excision repair, H) Nucleotide excision repair, and I) LPS synthesis. Reactivity of in vivo vs cell and cell-free cultures are always the same (except cbu0608, due to induction of mevalonate pathway in cell culture similar to in vivo, relative to cell-free culture system). 
encodes several generic transporters in its genome which could potentially transport these co-factors that are crucial for several enzyme reactions involved in amino acid, glucose, lipid metabolism or the regulation of genes.

As the fatty acid degradation pathway was up-regulated in vivo, catabolism of fatty acids appeared to play an important role in energy metabolism. Among the genes implicated in this pathway, the $y f c X$ gene was most strongly induced (Fig 5F). This results in anaerobic degradation of long/ medium chain fatty acids and produces NADH as a major cofactor for energy generation [46]. Genes involved in carbohydrate metabolism (dehydrogenases such as $p d h A, p d h C, \operatorname{lpd} A, \operatorname{sucB}, s d h A$, $s d h C$ ) were repressed under in vivo conditions, indicating an abundance of fatty acids (and fewer carbohydrates) in host cells. In cell and cell-free cultures these carbohydrate metabolism genes were enhanced, suggesting oxidation of glycolytic and TCA intermediates for energy production. The Cytochrome bd oxidase gene ( $c y d A$ ) was highly induced under in vivo conditions but at lower levels in cell culture, indicating an increased affinity for oxygen under intracellular conditions [47]. These differences suggest that the kind of nutrients found in the environment affects pathogen manipulation.

In conclusion, under in vivo conditions, the transportation of organic nutrients was upregulated, showing increased dependence of the bacterium on host-derived products. In vivo, the bacteria also shows enhanced biosynthetic capacities, which are directed to fulfill metabolic requirements that are unavailable directly from the host. In contrast, in vivo the bacterium represses a majority of genes coding for nucleotide synthesis and ribonucleotide reductases, which could be connected to the slow replication rate observed compared to in vitro culture conditions. Within in vitro cultures, gene regulation in cell culture were similar to in vivo but at much lower mRNA levels, indicating similar intracellular metabolic adaptations. Hence anaerobiosis, nutrient limitations, inaccessibility of co-factors were prominent environmental conditions encountered by C. burnetii during growth in vivo in the mice spleens.

\section{Enhanced regulation of transporter systems in hosts}

Bacterial transporters import essential nutrients, but are also used to import physiological substrates and export toxic molecules, both critical for cell viability, pathogenicity and virulence [48]. Under in vivo conditions, many transporters encoded by C. burnetii due to its intracellular life style were probably induced to maintain homeostasis [24]. Among these transporters are, sodium ion/proton exchangers (shaE, shaA, nhap.1), which might play a vital role in bacterial cytoplasmic $\mathrm{pH}$ homeostasis within the acidic PV. Several predicted drug-efflux systems (CBU0804, CBU0048, CBU0833, CBU1808, CBU1809) were enhanced, providing resistance and removal of toxins and host defensins. Among the metal transporters, gene implicated in inorganic phosphate transport were strongly up-regulated (CBU0014) under in vivo conditions along with up-regulation of phosphate starvation-responsive loci $p h o B R$, which is one of the few two-component regulatory system present in 
C. burnetii. Under phosphate starvation, enhanced expression of ATP producing genes was observed, possibly due to increased ATP demand caused by a limited amount of available phosphate [49].

\section{High degree of oxidative stress in host cells}

The generation of oxidative stress agents, which damage key bacterial components, is an innate defense response of host phagocytic cells. Strategies used by C. burnetii to avoid reactive substances mainly involve the enzymatic destruction of radicals and DNA repair mechanisms. Among ROS scavenging systems, peroxiredoxin $(a h p D)$ and its response regulator $(o x y R)$ genes were enhanced under in vivo conditions. Other ROS scavenging enzymes such as functional catalase (KatE) are present in only few C. burnetii strains. Presence or absence of a functional catalase gene in 602 was assessed by mapping its genome sequences against a strain containing a functional catalase gene (Cbuk_Q154, Accession number: NC_011528.1) as shown previously [15]. The genome sequences of 602 showed the presence of a highly truncated catalase gene as in NM [50]. The absence of a functional catalase gene in 602 and NM might be compensated by peroxiredoxins, which were enhanced under in vivo hosts. Under in vitro conditions, other ROS scavenging enzymes were enhanced in cell culture $(\operatorname{ahpC1}, \operatorname{ahpC2})$ and cell-free culture $(\operatorname{sod} C)$, thus indicating an increased peroxidase presence in intracellular hosts and superoxide radicals in the extracellular cell-free system. An induced protein synthesis was observed in the cell-free system, possibly leading to enhanced expression of the glutathione pathway, which restores protein function by reducing oxidized residues. Genes implicated in DNA repair (base excision repair, methyl mismatch repair and nucleotide excision repair) were strongly enhanced under in vivo conditions and collectively repair various DNA damages caused by oxidative stress (Fig 5G, 5H) [51-55]. Also the upregulation of addAB genes was seen; this is a novel adaptation of $C$. burnetii in its intracellular niche, which mediates DNA repair via homologous recombination [56]. Thus enhanced expression of DNA repair was seen in vivo and at relatively lower rate in cell culture, indicating bacterial response to increased oxidative stress in intracellular environments.

\section{Induction of virulence genes in vivo}

Among the known virulence factors, lipopolysaccharides (LPS) are considered to be the major determinant of the virulent phenotypic expression and infection of C. burnetii. Most likely the LPS shields the bacterial cell surface from innate immune recognition $[57,58]$ and/or the LPS phase-II works as an immunological decoy. Genes encoding structural features of LPS such as lipid A, the core region and the cluster of genes involved in $\mathrm{O}$-antigen synthesis were enhanced under in vivo conditions compared to in vitro systems (Fig 5I) [59]. ABC transporters implicated in lipid A (MsbA) and O-antigen (CBU0703) transport were strongly induced, indicating the export of LPS components to the outer membrane, where it plays an important role in bacterial virulence $[59,60]$. Within the in vitro models, the $\mathrm{p} 1$ and $\mathrm{p} 2$ cell-free cultures showed similar level of regulation of $\mathrm{O}$-antigen genes as 
in cell-culture, whereas the adapted cell-free culture (passage $n>10$ ) showed a significant downregulation of these genes, due to their deletion resulting in an on-going transformation into phase II forms [15]. Occurrence of phase II forms through the functional deletion of O-antigen coding genes might be an advantageous feature in the bacteria, allowing it to conserve the metabolic energy required to synthesize complex polysaccharides [61] and instead use energy for other important processes, such as growth or protein synthesis. This would be consistent with the enhanced expression of LPS genes seen in C. burnetii under in vivo conditions, and may be important during infection and increase in adaptation capabilities of the bacterium in hosts. Moreover, modifications in transcriptome of $C$. burnetii were seen only in the O-antigen region of high passaged cells, which might possibly result in reduced virulence potential.

Among the other virulence factors "Type IV secretion systems" (T4SS) have been identified as one of the important transfer systems in C. burnetii that delivers bacterial effector proteins into the host cytosol and are intimately involved in the pathogenesis of the bacterium. Genome sequencing has revealed the presence of an intact T4SS homologous to L. pneumophila Dot/Icm system. To date, only this system has been shown as a virulent determinant essential for the creation of vacuole, establishing a niche for intracellular survival and replication of the bacterium in eukaryotic host cells [24, 62-66]. Previous transcriptional studies on Dot/Icm system in $C$. burnetii has shown high expression of these genes during early stage infections $[67,68]$. Under in vivo conditions, the expression of Dot/Icm genes along with their transcription regulator ( $\mathrm{pmrA}$ ) were repressed with respect to in vitro models, probably due to later stages of growth of the bacterium in vivo where the infection has already taken place and the function of these genes had already been exerted, resulting in the establishment of replicative vacuole and a productive infection. Although, the transcription of these genes is repressed in later infection stages, decreased protein levels are maintained constantly [68]. Hence secretion of effector proteins of the T4SS required for maintaining an intracellular niche is still possible in the host cells. Under in vivo conditions, T4SS effector proteins such as ankyrin repeat domain containing proteins $(\operatorname{ankC}, a n k G)$ were up-regulated, and have been shown to interfere with the host cell apoptosis pathway [69]. Other effector proteins encoded by plasmid genes (CBU0014, CBU0015, CBU0006) were also up-regulated. These proteins are delivered by T4SS into host cytosol and might play a role in subversion of host cell functions [70]. Under in vitro conditions, the T4SS was upregulated. In cell culture, this system must be enhanced as the bacteria emerging from PV can re-infect new cells in the culture. Interestingly, this system was also seen to be enhanced in cell-free culture, although the bacterium does not require it as the medium is devoid of cells. Hence, the cell-free in vitro environment might induce the full repertoire of transcriptional responses required for successful pathogenesis of the bacterium for future challenges and transmission.

Others predicted virulence factors implicated in the manipulation of host-cell components and processes were also relatively enhanced under in vivo conditions. Among these were putative genes encoding phospholipase A (CBU0489) which can act on host phospholipids and generate lipid 
signaling [50,71], acid phosphatase (CBU0335) which can phosphorylate host proteins and decreases oxidative burst after phagocytosis of the bacterium [67], proteins with eukaryotic like domains (CBUA0014, CBU2078) which can modulate host ubiquitination pathways and disrupting host cell processes during infection [72, 73] and other eukaryotic like protein kinases similar to STPKs (CBU0175, CBU1379) which can directly interfere in host cell signal transduction [50, 74]. Hence, strong regulation of genes involved in manipulating and functionally mimicking the activity of host cell proteins was seen as a virulence property of the bacterium in hosts.

\section{Stringent responses of $C$. burnetii in hosts}

Under environmental stress (such as the intracellular in vivo conditions), bacterial species show stringent responses to escape unfavorable conditions. Under in vivo conditions, genes implicated in alarmone degradation were regulated (down-regulation of spoT and up-regulation of relA) which results in accumulation of unusual guanosine nucleotides (p)ppGpp, a hallmark of stringent response in several bacterial species [75]. In several bacterial species stringent responses were shown to play a role in sporulation, virulence and long-term persistence, usually in response to environmental stress $[75,76]$. For example, in L. pneumophila, a close relative of C. burnetii, the stringent pathways are important factors in modulating the virulent attributes that help its survival in the host [77]. The role of these regulatory networks in $C$.burnetii is not known yet and differential regulation of these genes under in vivo conditions at later infection stages (Day 20) may result in a greater benefit to the survival of the bacteria, which would be an interesting area of study in need of further investigation.

Additional microarray experiments performed in parallel with NM reference strain revealed similar gene expression patterns in vivo compared to in vitro models (data not shown) indicating that the trends described by the Dutch 602 outbreak field strain are likely to prove relevant to other C. burnetii strains more generally. Therefore, the microarray data increases our knowledge regarding pathogen adaptation to host micro environments, selective pressures in these environments and bacterial factors responsible for evading host responses. Finally, the microarray data can be used as a guide to screen and select genes encoding putative membrane proteins and other proteins that have antigenic properties among the differentially regulated genes during varying conditions. This might provide a framework for new possibilities to investigate and identify virulent factors important in the design of vaccines.

\section{Conclusions}

This is the first report on C. burnetii genome-wide transcriptome profiles isolated from in vivo and in vitro culture models. The data provide insight into pathways associated with bacterial adaptation to varying environmental conditions and identify potential virulent factors based on the mRNA levels of genes measured by microarray approach. Transcriptome differences in C. burnetii were profound 
between in vivo and in vitro culture models. Within the in vitro culture systems, the expression profile of C. burnetii from cell culture was closely related to expression profiles from the in vivo model (Fig 2). This might be due to its intracellular nature and low nutrient availability in cell culture compared to free availability of nutrients in the more artificial cell-free culture model. The cell-free culture adapting passages ( $\mathrm{p} 1$ and $\mathrm{p} 2$ ) expression profiles migrated towards cell-free adapted strains indicating the ability of the bacterium to adapt promptly to available resources in the environment.

Between the different models, major differential gene regulations were primarily observed in metabolic processes of the bacteria. Under in vivo conditions, the exciting observations in our study were; enhanced expression of anionic phospholipid coding gene $(c l s)$ for maintenance of acidic $\mathrm{pH}$ at outer membrane surface; organic molecule transportation indicating non-starvation of the bacteria; phosphate limitation as an important intracellular signal, and enhanced DNA repair mechanisms to combat against oxidative stress in host cells. We have also been able to provide evidence that based on the observed gene regulation; lipids play a very important role in several biosynthetic processes of $C$. burnetii in vivo and are the primary sources for anaerobic energy metabolism, possibly due to their abundant presence in mice spleens. However, carbohydrate metabolism was observed under in vitro culture systems indicating type and availability of nutrients were important determinants for resulting expression patterns of $C$. burnetii in different models. Relative to in vitro conditions, the in vivo environment significantly enhanced expression of virulence genes such as LPS-linked genes and factors implicated in host-cell modulations (inhibition of apoptosis, evading defense mechanisms etc.). This capability to modulate host response to infection seems to be a key factor for C. burnetii survival in vivo.

\section{Materials and methods}

\section{Bacterial strains and culture models}

C. burnetii strain 602 was isolated from aborted placenta of goats during a $\mathrm{Q}$ fever outbreak and initially propagated on Buffalo Green Monkey (BGM) cells (European Collection of Cell Cultures, Salisbury) as described previously $[15,20]$. The strain was genotyped by MLVA as CbNL01, a predominant genotype during the outbreak. The 602 and Nine Mile RSA493 (NM) reference strain were propagated in different models explained below.

\section{Mice spleen model}

The spleens from mice infected with C. burnetii 602 and NM strains were used as in vivo model to study bacterial gene expressions. Animal experiments were conducted using 7 week old SpecificPathogen-Free Swiss female OF1 mice (Charles River, l'Arbresle). The mice were housed under sterile conditions in biosecurity level 3 facilities. After a week of acclimatization, infections were performed with 3 Swiss OF1 mice per strain. The mice were inoculated intraperitoneally with $0.2 \mathrm{ml}$ PBS suspension of C. burnetii (grown in BGM cell culture) with each mouse receiving a dose of $10^{7}$ 
genome copy equivalents as determined by quantitative PCR [20]. Twenty days after inoculation the mice were euthanized, spleens were harvested aseptically and immediately frozen in liquid nitrogen and then at $-80^{\circ} \mathrm{C}$ for RNA isolation. Animal experiments were approved by the animal experiment commission of the Central Veterinary Institute of Wageningen UR, and conducted in accordance with the Dutch regulations on animal experimentation.

\section{In vitro culture Models}

The C. burnetii strains used in the study were propagated in cell and cell-free cultures in triplicate from the low passage stock cultures stored at $-80^{\circ} \mathrm{C}$.

\section{Cell culture model}

For the cell culture model, BGM cells with EMEM culture media without antibiotics $(10 \%$ bovine serum albumin, 1\% NEAA, $1 \%$ glutamax) were used to propagate C. burnetii from stock cultures (passage $\mathrm{n}=10-12$ in BGM cell culture) until stationary growth phase [20].

\section{Cell-free culture model}

For the cell-free culture, the protocol previously described [23] was used to propagate C. burnetii (passage $n=10-12$ in cell-free culture) until stationary growth phase. Two serial passages ( $p 1$ and $p 2$ ) of the strains from cell to cell-free culture model were also performed in addition and were included in the study.

\section{C. burnetii specific RNA isolation}

The frozen spleens were thawed on ice and a piece of the tissue was homogenized (PowerGen 125, Fischer Scientific, Pittsburgh) in trizol reagent (Ambion, Austin) with a disposable plastic micro pestle (OMNI-Tip plastic homogenizing probes, OMNI international, Kennesaw) and used for RNA isolation. The in vitro cell culture supernatants were centrifuged at $1200 \mathrm{rpm}, 4^{\circ} \mathrm{C}$ for $10 \mathrm{~min}$ to remove cell debris. These processed supernatants of cell and the cell-free cultures were centrifuged at $16000 \mathrm{rpm}, 4^{\circ} \mathrm{C}$ for $30 \mathrm{~min}$ to pellet the bacterial cells and subsequently treated with trizol for RNA isolation. Total RNA was isolated by using Direct-zol RNA MiniPrep Kit (Zymo Research, Irvine) as per manufacturer's instructions. All samples were DNase treated prior to RNA isolation. The host RNA from total spleen RNA was selectively removed by MICROBEnrich kit (Ambion, Austin) followed by RNA clean and Concentrator-5 kit (Zymo Research, Irvine) for RNA clean-up and concentration.

\section{C. burnetii specific microarray}

Custom gene expression microarrays, $8 \times 15 \mathrm{~K}$, designed by Agilent (Agilent Technologies, Santa Clara), consisted of eight arrays per slide with duplicates of 7500 probes. All the probes were user- 
designed by eArray web-based probe design tool (https://earray.chem.agilent.com/earray) based on the complete gene repertoire of C. burnetii (NCBI, Accession numbers: AE016828.2, CP000733.1, CP001020.1, CP001019.1, CP001021.1 and draft genomes of Dutch C. burnetii strains). 1 to 4 probes of $120 \mathrm{bp}$ in length were designed per gene such that the probes evenly distributed across the gene.

\section{RNA amplification, labelling and hybridization}

Poly A tails were tagged to purified bacterial total RNA (500 ng) from all the culture models with a Poly A Polymerase Tailing kit (Epicenter Illumina company, Madison) as per manufacture instructions. Poly A tailed RNA (250 ng) were prepared for amplification and labelling using the Low Input Quick Amp Labeling Kit from Agilent following the detailed kit protocol. Cyanine 3-labelled cRNA was purified using RNeasy mini kit (Qiagen, Hilden). Labelled cRNA was examined with the Nanodrop ND-1000 (Thermo Fisher Scientific, Wilmington) to assess its concentration and quality. Hybridization and post-hybridization washes were conducted according to Agilent's one-color microarray-based gene expression analysis protocol (Version G4140_90042).

\section{Data and statistical analysis}

Microarray slides were scanned using a SureScan High Resolution DNA microarray scanner (Agilent Technologies, Santa Clara). Data was obtained through Agilent's feature extraction software (Version 10.7.3.1) and loaded in GeneSpring GX (Version 12.6.1) where signal intensity was $\log _{2}$-transformed and median normalized for all triplicate samples before analysis. PCA plots (based on correlation coefficients obtained from pair-wise comparisons of samples) and clustering of microarray data (based on Euclidian distance and Ward's linkage parameters) were analyzed using Genespring GX to display relative differences across samples. Differentially regulated genes between different culture models were determined by 2-way ANOVA $(p<0.05$, adjusted by the Benjamini and Hochberg false discovery rate algorithm) and a cut-off of 2 for the fold change was identified as significantly differential expression. These differentially regulated genes from different comparisons were plotted by means of a Venn diagram using Vennerable package (Version 2.0). David (http://david.abcc.ncifcrf.gov/) and KeggArray software (http://www.genome.jp/kegg/) was used for pathway analysis and to obtain $P$ values of significantly regulated pathways for each comparison of 602 and NM strains against Coxiella burnetii RSA 493 (organism code: cbu) KEGG pathway database.

\section{Acknowledgements}

We thank the animal care takers and Frank Harders for their technical support. This work was supported by the Dutch Ministry of Economic Affairs WOT-01-002-005.06, WOT-01-002-005.05, KB-12-005.01-019, and KB-12-005.01-005. 


\section{References}

1. Maurin M, Raoult D. Q fever. Clin Microbiol Rev. 1999; 12(4):518-53.

2. Woldehiwet Z. Q fever (coxiellosis): epidemiology and pathogenesis. Res Vet Sci. 2004; 77(2):93-100.

3. Norlander L. Q fever epidemiology and pathogenesis. Microbes Infect Inst Pasteur. 2000; 2(4):417-24.

4. Arricau-Bouvery $\mathrm{N}$, Rodolakis $\mathrm{A}$. Is $\mathbf{Q}$ fever an emerging or re-emerging zoonosis?. Vet Res. 2005; 36(3):327-49.

5. Raoult D, Marrie T, Mege J. Natural history and pathophysiology of $\mathbf{Q}$ fever. Lancet Infect Dis. 2005; 5(4):219-26.

6. Mazokopakis EE, Karefilakis CM, Starakis IK. Q fever endocarditis. Infect Disord Drug Targets. 2010; 10(1):27-31.

7. Roest HIJ, Ruuls RC, Tilburg JJHC, Nabuurs-Franssen MH, Klaassen CHW, Vellema P, et al. Molecular epidemiology of Coxiella burnetii from ruminants in $Q$ fever outbreak, the Netherlands. Emerg Infect Dis. 2011; 17(4):668-75.

8. Roest HIJ, Tilburg JJHC, van der Hoek W, Vellema P, van Zijderveld FG, Klaassen CHW, et al. The $\mathbf{Q}$ fever epidemic in The Netherlands: history, onset, response and reflection. Epidemiol Infect. 2011; 139(1):1-12.

9. Enserink M. Questions Abound in Q-Fever Explosion in the Netherlands. Science. 2010; 327(5963):266-7.

10. Kampschreur LM, Hagenaars JCJP, Wielders CCH, Elsman P, Lestrade PJ, Koning OHJ, et al. Screening for Coxiella burnetii seroprevalence in chronic $Q$ fever high-risk groups reveals the magnitude of the Dutch $\mathbf{Q}$ fever outbreak. Epidemiol Infect. 2013; 141(4):847-51.

11. Van der Hoek W, Dijkstra F, Schimmer B, Schneeberger PM, Vellema P, Wijkmans C, et al. Q fever in the Netherlands: an update on the epidemiology and control measures. Euro Surveill. 2010; 15(12):19520.

12. Akporiaye ET, Rowatt JD, Aragon AA, Baca OG. Lysosomal response of a murine macrophage-like cell line persistently infected with Coxiella burnetii. Infect Immun. 1983; 40(3):1155-62.

13. Kersh GJ, Lambourn DM, Self JS, Akmajian AM, Stanton JB, Baszler TV, Raverty SA, Massung RF. Coxiella burnetii Infection of a Steller Sea Lion (Eumetopias jubatus) Found in Washington State. J Clin Microbiol. 2010; 48(9):3428-31.

14. Omsland A, Cockrell DC, Howe D, Fischer ER, Virtaneva K, Sturdevant DE, et al. Host cell-free growth of the $\mathbf{Q}$ fever bacterium Coxiella burnetii. Proc Natl Acad Sci. 2009; 106(11):4430-34.

15. Kuley R, Smith HE, Frangoulidis D, Smits MA, Jan Roest HI, Bossers A. Cell-Free Propagation of Coxiella burnetii Does Not Affect Its Relative Virulence. PLOS ONE. 2015; 10:e48949

16. Scott GH, Williams JC, Stephenson EH. Animal models in Q fever: pathological responses of inbred mice to phase I Coxiella burnetii. J Gen Microbiol. 1987; 133(3):691-700. 
17. Andoh M, Zhang G, Russell-Lodrigue KE, Shive HR, Weeks BR, Samuel JE. T Cells Are Essential for Bacterial Clearance, and Gamma Interferon, Tumor Necrosis Factor Alpha, and B Cells Are Crucial for Disease Development in Coxiella burnetii Infection in Mice. Infect Immun. 2007; 75(7):3245-55.

18. Russell-Lodrigue KE, Andoh M, Poels MWJ, Shive HR, Weeks BR, Zhang GQ, et al. Coxiella burnetii isolates cause genogroup-specific virulence in mouse and guinea pig models of acute $\mathbf{Q}$ fever. Infect Immun. 2009; 77(12):5640-50.

19. Kazár J, Lesý M, Propper P, Valková D, Brezina R. Comparison of virulence for guinea pigs and mice of different Coxiella burnetii phase I strains. Acta Virol. 1993; 37(6):437-48.

20. Roest H-J, van Gelderen B, Dinkla A, Frangoulidis D, van Zijderveld F, Rebel J, et al. Q Fever in Pregnant Goats: Pathogenesis and Excretion of Coxiella burnetii. PLoS ONE. 2012; 7:e48949.

21. Lever MS, Bewley KR, Dowsett B, Lloyd G. In vitro susceptibility of Coxiella burnetii to azithromycin, doxycycline, ciprofloxacin and a range of newer fluoroquinolones. Int $J$ Antimicrob Agents. 2004; 24(2):194-6.

22. Maurin M, Benoliel AM, Bongrand P, Raoult D. Phagolysosomes of Coxiella burnetii-infected cell lines maintain an acidic pH during persistent infection. Infect Immun. 1992; 60(12):5013-6.

23. Omsland A, Beare PA, Hill J, Cockrell DC, Howe D, Hansen B, et al. Isolation from animal tissue and genetic transformation of Coxiella burnetii are facilitated by an improved axenic growth medium. Appl Environ Microbiol. 2011; 77(11):3720-5.

24. Seshadri R, Paulsen IT, Eisen JA, Read TD, Nelson KE, Nelson WC, et al. Complete genome sequence of the Q-fever pathogen Coxiella burnetii. Proc Natl Acad Sci. 2003; 100(9):5455-60.

25. Alsaleh A, Pellerin J-L, Rodolakis A, Larrat M, Cochonneau D, Bruyas J-F, et al. Detection of Coxiella burnetii, the agent of $Q$ fever, in oviducts and uterine flushing media and in genital tract tissues of the non pregnant goat. Comp Immunol Microbiol Infect Dis. 2011; 34(4):355-60.

26. Mori M, Boarbi S, Michel $\mathrm{P}$, Bakinahe R, Rits $\mathrm{K}$, Wattiau $\mathrm{P}$, et al. In Vitro and In Vivo Infectious Potential of Coxiella burnetii: A Study on Belgian Livestock Isolates. PLoS ONE. 2013; 8:e67622.

27. Benson J: Developing a murine model for $\mathbf{Q}$ fever. In: Effect of inoculation route on bacterial infection of tissues and development of histopathological lesions in mice infected with Coxiella burnetii. Colorado State University, Colorado; 2011: p.28-48.

28. Tatusov RL, Galperin MY, Natale DA, Koonin EV: The COG database: a tool for genome-scale analysis of protein functions and evolution. Nucleic Acids Res. 2000; 28(1):33-6.

29. Paretsky D, Consigli RA, Downs CM: Studies on the physiology of Rickettsiae III. Glucose Phosphorylation and Hexokinase Activity in Coxiella burnetii. J Bacteriol. 1962; 83(3):538-43.

30. Gilk SD. Role of Lipids in Coxiella burnetii Infection. In Coxiella burnetii: Recent Advances and New Perspectives in Research of the Q Fever Bacterium. Toman R, Heinzen RA, Samuel JE, Mege J-L, editors. Springer, Netherlands; 2012: p.199-213.

31. Card GL, Trautman JK. Role of anionic lipid in bacterial membranes. Biochim Biophys Acta. 1990; 1047(1):77-82. 
32. Domingues P, Palkovic P, Toman R. Analysis of phospholipids from Coxiella burnetii by fast atom bombardment mass spectrometry. A rapid method for differentiation of virulent phase I and low virulent phase II cells. Acta Virol. 2002; 46(2):121-4.

33. Smith PB, Snyder AP, Harden CS. Characterization of bacterial phospholipids by electrospray ionization tandem mass spectrometry. Anal Chem. 1995; 67(11):1824-30.

34. Boucher Y, Doolittle WF. The role of lateral gene transfer in the evolution of isoprenoid biosynthesis pathways. Mol Microbiol. 2000; 37(4):703-16.

35. Lee YH, Helmann JD. Reducing the Level of Undecaprenyl Pyrophosphate Synthase Has Complex Effects on Susceptibility to Cell Wall Antibiotics. Antimicrob Agents Chemother. 2013; 57(9):4267-75.

36. Okada K, Minehira M, Zhu X, Suzuki K, Nakagawa T, Matsuda H, et al. The ispB gene encoding octaprenyl diphosphate synthase is essential for growth of Escherichia coli. J Bacteriol. 1997; 179(9):3058-60.

37. Gilk SD, Cockrell DC, Luterbach C, Hansen B, Knodler LA, Ibarra JA, et al. Bacterial Colonization of Host Cells in the Absence of Cholesterol. PLoS Pathog. 2013; 9:e1003107.

38. Gilk SD, Beare PA, Heinzen RA. Coxiella burnetii expresses a functional $\Delta \mathbf{2 4}$ sterol reductase. J Bacteriol. 2010; 192(23):6154-9.

39. Howe D, Heinzen RA. Coxiella burnetii inhabits a cholesterol-rich vacuole and influences cellular cholesterol metabolism. Cell Microbiol. 2006; 8(3):496-507.

40. Catron DM, Lange Y, Borensztajn J, Sylvester MD, Jones BD, Haldar K. Salmonella enterica Serovar Typhimurium Requires Nonsterol Precursors of the Cholesterol Biosynthetic Pathway for Intracellular Proliferation. Infect Immun. 2004; 72(2):1036-42.

41. Xiong Q, Lin M, Rikihisa Y. Cholesterol-dependent anaplasma phagocytophilum exploits the low-density lipoprotein uptake pathway. PLoS Pathog. 2009; 5:e1000329.

42. Wu C, Miloslavskaya I, Demontis S, Maestro R, Galaktionov K. Regulation of cellular response to oncogenic and oxidative stress by Seladin-1. Nature. 2004; 432(7017):640-5.

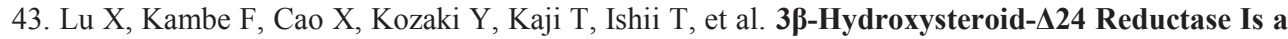
Hydrogen Peroxide Scavenger, Protecting Cells from Oxidative Stress-Induced Apoptosis. Endocrinology. 2008; 149(7):3267-73.

44. Xie G, Bonner CA, Jensen RA. Dynamic diversity of the tryptophan pathway in chlamydiae: reductive evolution and a novel operon for tryptophan recapture. Genome Biol. 2002; 3(9):0051.1-0051.17.

45. Fehlner-Gardiner C, Roshick C, Carlson JH, Hughes S, Belland RJ, Caldwell HD, et al. Molecular Basis Defining Human Chlamydia trachomatis Tissue Tropism. A possible role for tryptophan synthase. J Biol Chem. 2002; 277(30):26893-903.

46. Muñoz-Elías EJ, McKinney JD. Carbon metabolism of intracellular bacteria. Cell Microbiol. 2006; 8(1):10-22.

47. Cotter PA, Chepuri V, Gennis RB, Gunsalus RP. Cytochrome o (cyoABCDE) and d (cydAB) oxidase gene expression in Escherichia coli is regulated by oxygen, $\mathrm{pH}$, and the fnr gene product. J Bacteriol. 1990; 172(11):6333-8. 
48. Davidson AL, Dassa E, Orelle C, Chen J. Structure, Function, and Evolution of Bacterial ATPBinding Cassette Systems. Microbiol Mol Biol. Rev MMBR 2008; 72(2):317-64.

49. Marzan LW, Shimizu K. Metabolic regulation of Escherichia coli and its phoB and phoR genes knockout mutants under phosphate and nitrogen limitations as well as at acidic condition. Microb Cell Factories. 2011; 10(1):39.

50. Beare PA, Unsworth N, Andoh M, Voth DE, Omsland A, Gilk SD, et al. Comparative genomics reveal extensive transposon-mediated genomic plasticity and diversity among potential effector proteins within the genus Coxiella. Infect Immun. 2009; 77(2):642-56.

51. Aamodt RM, Falnes PØ, Johansen RF, Seeberg E, Bjørås M. The Bacillus subtilis counterpart of the mammalian 3-methyladenine DNA glycosylase has hypoxanthine and 1,N6-ethenoadenine as preferred substrates. J Biol Chem. 2004; 279(14):13601-6.

52. Grzesiuk E, Gozdek A, Tudek B. Contribution of E. coli AlkA, TagA glycosylases and UvrABC-excinuclease in MMS mutagenesis. Mutat Res. 2001; 480-481:77-84.

53. Wang G, Alamuri P, Humayun MZ, Taylor DE, Maier RJ. The Helicobacter pylori MutS protein confers protection from oxidative DNA damage. Mol Microbiol. 2005; 58(1):166-76.

54. Cabusora L, Sutton E, Fulmer A, Forst CV. Differential network expression during drug and stress response. Bioinforma Oxf Engl. 2005; 21(12):2898-905.

55. Park S-H, Lee H-W, Cao W. Screening of nitrosative stress resistance genes in Coxiella burnetii: Involvement of nucleotide excision repair. Microb Pathog. 2010; 49(6):323-9.

56. Mertens K, Lantsheer L, Ennis DG, Samuel JE. Constitutive SOS expression and damageinducible AddAB-mediated recombinational repair systems for Coxiella burnetii as potential adaptations for survival within macrophages. Mol Microbiol. 2008; 69(6):1411-26.

57. Moos A, Hackstadt T. Comparative virulence of intra- and interstrain lipopolysaccharide variants of Coxiella burnetii in the guinea pig model. Infect Immun. 1987; 55(5):1144-50.

58. Shannon JG, Howe D, Heinzen RA. Virulent Coxiella burnetii does not activate human dendritic cells: Role of lipopolysaccharide as a shielding molecule. Proc Natl Acad Sci. 2005; 102(24):8722-7.

59. Hoover TA, Culp DW, Vodkin MH, Williams JC, Thompson HA. Chromosomal DNA deletions explain phenotypic characteristics of two antigenic variants, phase II and RSA 514 (crazy), of the Coxiella burnetii nine mile strain. Infect Immun. 2002; 70(12):6726-33.

60. Zhou Z, White KA, Polissi A, Georgopoulos C, Raetz CR. Function of Escherichia coli MsbA, an essential $\mathrm{ABC}$ family transporter, in lipid $\mathrm{A}$ and phospholipid biosynthesis. $\mathrm{J}$ Biol Chem. 1998; 273(20):12466-75.

61. Hackstadt T, Peacock MG, Hitchcock PJ, Cole RL. Lipopolysaccharide variation in Coxiella burnetti: intrastrain heterogeneity in structure and antigenicity. Infect Immun. 1985; 48(2):35965 .

62. Van Schaik EJ, Chen C, Mertens K, Weber MM, Samuel JE. Molecular pathogenesis of the obligate intracellular bacterium Coxiella burnetii. Nat Rev Microbiol. 2013; 11(8):561-73.

63. Newton HJ, McDonough JA, Roy CR. Effector Protein Translocation by the Coxiella burnetii Dot/Icm Type IV Secretion System Requires Endocytic Maturation of the Pathogen-Occupied Vacuole. PLOS ONE. 2013; 8:e54566. 
64. Kagan JC, Roy CR. Legionella phagosomes intercept vesicular traffic from endoplasmic reticulum exit sites. Nat Cell Biol. 2002; 4(12):945-54.

65. Swanson MS, Isberg RR. Association of Legionella pneumophila with the macrophage endoplasmic reticulum. Infect Immun. 1995; 63(9):3609-20.

66. Hubber A, Roy CR. Modulation of host cell function by Legionella pneumophila type IV effectors. Annu Rev Cell Dev Biol. 2010; 26:261-83.

67. Coleman SA, Fischer ER, Howe D, Mead DJ, Heinzen RA. Temporal analysis of Coxiella burnetii morphological differentiation. J Bacteriol. 2004; 186(21):7344-52.

68. Morgan JK, Luedtke BE, Thompson HA, Shaw EI. Coxiella burnetii type IVB secretion system Region I genes are expressed early during infection of host cells. FEMS Microbiol Lett. 2010; 311(1):61-9.

69. Lührmann A, Nogueira CV, Carey KL, Roy CR. Inhibition of pathogen-induced apoptosis by a Coxiella burnetii type IV effector protein. Proc Natl Acad Sci. 2010; 107(44):18997-19001.

70. Voth DE, Beare PA, Howe D, Sharma UM, Samoilis G, Cockrell DC, et al. The Coxiella burnetii Cryptic Plasmid Is Enriched in Genes Encoding Type IV Secretion System Substrates. J Bacteriol. 2011; 193(7):1493-1503.

71. Wymann MP, Schneiter R. Lipid signalling in disease. Nat Rev Mol Cell Biol. 2008; 9(2):162176.

72. Roy CR, Mukherjee S. Bacterial FIC Proteins AMP Up Infection. Sci Signal. 2009; 2(62):pe14.

73. Yarbrough ML, Li Y, Kinch LN, Grishin NV, Ball HL, Orth K. AMPylation of Rho GTPases by Vibrio VopS disrupts effector binding and downstream signaling. Science. 2009; 323(5911):26972 .

74. Walburger A, Koul A, Ferrari G, Nguyen L, Prescianotto-Baschong C, Huygen K, et al. Protein kinase $\mathbf{G}$ from pathogenic mycobacteria promotes survival within macrophages. Science. 2004; 304(5678): $1800-4$.

75. Chatterji D, Kumar Ojha A. Revisiting the stringent response, ppGpp and starvation signaling. Curr Opin Microbiol. 2001; 4(2):160-5.

76. Carneiro S, Lourenço A, Ferreira EC, Rocha I. Stringent response of Escherichia coli: revisiting the bibliome using literature mining. Microb Inform Exp. 2011; 1(1):14.

77. Hammer BK, Swanson MS. Co-ordination of legionella pneumophila virulence with entry into stationary phase by ppGpp. Mol Microbiol. 1999; 33(4):721-31. 


\section{Supplementary files}

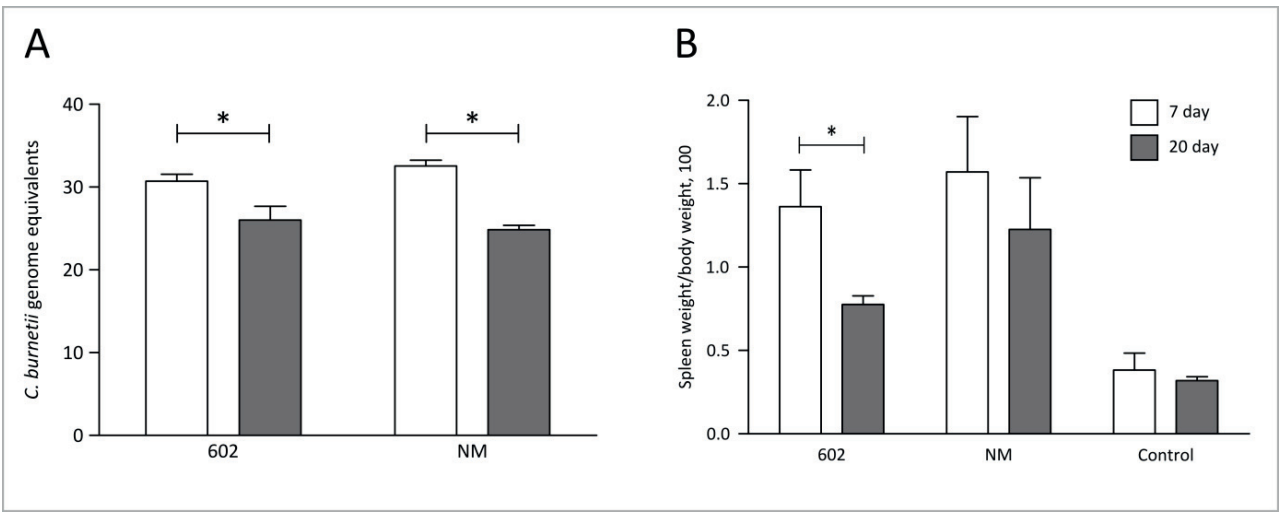

Figure S1. Changes in splenomegaly and bacterial genome equivalents present in the spleens of mice infected with 602 and NM C. burnetii strains at 7 and 20 days p.i. A) Bacterial genome equivalents of total spleens are evaluated by qPCR quantification and expressed as $\log 2$ transformed values, B) Degree of splenomegaly are expressed as the percentage of spleen weight compared with the body weight. The results are indicated as means \pm standard deviation, * indicates p-values smaller than 0.05 .

Table S1. List of genes differentially regulated in vivo and cell-free culture with respect to cell culture in vitro model of 602 strain, after passing statistical significance filter and showing more than two fold change in expression. All the genes are arranged based on COG functions. A positive value indicates that the gene is upregulated, a negative value indicates that the gene is down-regulated and - indicates no difference compared to cell culture in gene regulation under the respective condition.

http://bmcgenomics.biomedcentral.com/articles/10.1186/s12864-015-2143-7 



\section{Chapter 4}

First complete genome sequence of

the Dutch veterinary Coxiella burnetii strain NL3262, originating from the largest global $Q$ fever outbreak, and draft genome sequence of its epidemiologically-linked chronic human isolate NLhu3345937

Runa Kuley, Hilde E. Smith, Ingmar Janse, Frank Harders, Frank Baas, Elio Schijlen, Marrigje H Nabuurs-Franssen, Mari A Smits, Hendrik I.J. Roest, and Alex Bossers

Genome Announcements. 2016 Apr 21;4(2) 


\section{Abstract}

The largest global Q-fever outbreak occurred in the Netherlands during 2007-2010. Goats were identified as the major source of disease. Here we report the first complete genome sequence of $C$. burnetii goat outbreak strain NL3262 and that of an epidemiologically-linked chronic human strain, both having the outbreak-related CbNL01 MLVA genotype. 
Q fever is a zoonotic disease caused by Coxiella burnetii. Starting in 2007, Netherlands has been confronted with the largest global Q fever outbreak ever, involving 4026 human cases. Based on epidemiological and genotyping studies dairy goats and sheep were identified as the main source of the human Q fever outbreak. Special attention was given to the Dutch outbreak-specific strain of CbNL01 MLVA-genotype which was identified in abortive dairy goats as well as in humans (1-6). Current project was performed with C. burnetii strains of this predominant genotype isolated from an aborted goat placenta $(1,7)$ and from a heart valve of chronic Q fever patient during the outbreak period. Genome determination of the veterinary NL3262 strain is crucial for understanding the large outbreak and biology of this high virulent strain. The genomes of virulent veterinary (NL3262) and related human (NLhu3345937) outbreak strains were sequenced and their reconstructed genomes were compared to examine similarities and differences in their genome structures.

Strains NL3262 and NLhu3345937 were cultivated axenically in ACCM-2 media and BGMcells respectively $(7,8)$. Genomic DNA was isolated by phenol-chloroform method (9). A prior DNase treatment was performed to the bacterial pellet of NLhu3345937 to eliminate most host-derived DNA. The genome of NL3262 was de novo reconstructed from mixed assemblies using a combination of PacBio RS, Roche 454XL and Illumina PE250 MiSeq reads. Reconstructed genomes were improved with different Illumina read sets with a total average coverage of $600 \mathrm{X}$ using Pilon-1.8 (10). NLhu3345937 genome was de novo reconstructed using Illumina PE250 MiSeq reads using SPAdes3.6.2 (11) with a total average coverage of $285 \mathrm{X}$.

The complete NL3262 and draft genome sequence of NLhu3345937 contains 2,093,477 and 2,088,566 bp with $\mathrm{G}+\mathrm{C}$ content of 42.9 and $42.6 \%$ respectively. Genome comparisons of NL3262, NLhu3345937 and the draft NL-Limburg outbreak strains (12) using Nucmer (13), MUMi-BioNJ tree (14) and visualised using Artemis Comparison Tool (ACT; (15)) show that these strains are closely related with differences mainly based on single base pair mutations. Our veterinary and human strains both contain around the $37 \mathrm{~kb}$ pQpH1 plasmid similar to reference NM-RSA493 strain. Genome annotation of NL3262 by NCBI Prokaryotic Genome Annotation Pipeline (http://www.ncbi.nlm.nih.gov/genome/annotation prok) showed 2,101 coding sequences (CDS), 180 pseudogenes, 42 tRNA's, 3 rRNAs and 1 ncRNA. Compared to NM (accession no-NC_002971.3) the majority of genes (84\%) are orthologs found in both strains with only $16 \%$ of specific genes in NL3262 comprising mainly hypothetical products. Genome reconstruction was cumbersome between different passages of each strain due to high genome complexity as described before for other isolates (many transposons, repetitive IS1111 elements and rearrangements) (16). Observed high similarity between veterinary (NL3262) and human (NLhu3345937 and NL-Limburg) strains confirms the previous epidemiological and genotypic studies linking the goat and human infections during the Netherlands Q fever outbreak. A detailed comparative genome analysis of several sequenced $C$. burnetii isolates from different origins is ongoing. 


\section{Nucleotide sequence accession numbers}

C. burnetii NL3262 and NLhu3345937 genome and plasmid sequences have been deposited in GenBank under accession No. CP013667, CP0136678, CP014354 and CP014355 respectively.

\section{Acknowledgements}

We thank Annemieke Dinkla, Robin Ruuls for technical support and Marja E Jakobs from department of genome analysis, Amsterdam Medical Centre, for assistance in Roche-454 sequencing. We also thank the Dutch Food and Consumer Product Safety Authority for procuring the goat tissue specimen. 


\section{References}

1. Roest HIJ, Ruuls RC, Tilburg JJHC, Nabuurs-Franssen MH, Klaassen CHW, Vellema P, van den Brom R, Dercksen D, Wouda W, Spierenburg MAH, van der Spek AN, Buijs R, de Boer AG, Willemsen PTJ, van Zijderveld FG. 2011. Molecular epidemiology of Coxiella burnetii from ruminants in Q fever outbreak, the Netherlands. Emerg Infect Dis 17:668-675.

2. Van Steenbergen JE, Morroy G, Groot C a. R, Ruikes FGH, Marcelis JH, Speelman P. 2007. An outbreak of Q fever in The Netherlands--possible link to goats. Ned Tijdschr Geneeskd 151:1998-2003.

3. Wouda W, Dercksen DP. 2007. Abortion and stillbirth among dairy goats as a consequence of Coxiella burnetii. Tijdschr Diergeneeskd 132:908-911.

4. Karagiannis I, Schimmer B, Van Lier A, Timen A, Schneeberger P, Van Rotterdam B, De Bruin A, Wijkmans C, Rietveld A, Van Duynhoven Y. 2009. Investigation of a Q fever outbreak in a rural area of The Netherlands. Epidemiol Infect 137:1283-1294.

5. Karagiannis I, Morroy G, Rietveld A, Horrevorts AM, Hamans M, Francken P, Schimmer B. 2007. Q fever outbreak in the Netherlands: a preliminary report. Euro Surveill Bull Eur Sur Mal Transm Eur Commun Dis Bull 12:E070809.2.

6. Tilburg JJHC, Roest H-JIJ, Buffet S, Nabuurs-Franssen MH, Horrevorts AM, Raoult D, Klaassen CHW. 2012. Epidemic Genotype of Coxiella burnetii among Goats, Sheep, and Humans in the Netherlands. Emerg Infect Dis 18:887-889.

7. Roest H-J, van Gelderen B, Dinkla A, Frangoulidis D, van Zijderveld F, Rebel J, van Keulen L. 2012. Q Fever in Pregnant Goats: Pathogenesis and Excretion of Coxiella burnetii. PLoS ONE 7:e48949.

8. Omsland A, Beare PA, Hill J, Cockrell DC, Howe D, Hansen B, Samuel JE, Heinzen RA. 2011. Isolation from Animal Tissue and Genetic Transformation of Coxiella burnetii Are Facilitated by an Improved Axenic Growth Medium ${ }^{\nabla}$. Appl Environ Microbiol 77:3720-3725.

9. Kuley R, Smith HE, Frangoulidis D, Smits MA, Jan Roest HI, Bossers A. 2015. Cell-Free Propagation of Coxiella burnetii Does Not Affect Its Relative Virulence. PLoS ONE 10.

10. Walker BJ, Abeel T, Shea T, Priest M, Abouelliel A, Sakthikumar S, Cuomo CA, Zeng Q, Wortman J, Young SK, Earl AM. 2014. Pilon: An Integrated Tool for Comprehensive Microbial Variant Detection and Genome Assembly Improvement. PLoS ONE 9:e112963.

11. Bankevich A, Nurk S, Antipov D, Gurevich AA, Dvorkin M, Kulikov AS, Lesin VM, Nikolenko SI, Pham S, Prjibelski AD, Pyshkin AV, Sirotkin AV, Vyahhi N, Tesler G, Alekseyev MA, Pevzner PA. 2012. SPAdes: A New Genome Assembly Algorithm and Its Applications to Single-Cell Sequencing. J Comput Biol 19:455-477.

12. Hammerl JA, Mertens K, Sprague LD, Hackert VH, Buijs J, Hoebe CJ, Henning K, Neubauer H, Al Dahouk S. 2015. First Draft Genome Sequence of a Human Coxiella burnetii Isolate, Originating from the Largest Q Fever Outbreak Ever Reported, the Netherlands, 2007 to 2010. Genome Announc 3.

13. Kurtz S, Phillippy A, Delcher AL, Smoot M, Shumway M, Antonescu C, Salzberg SL. 2004. Versatile and open software for comparing large genomes. Genome Biol 5:R12.

14. Gascuel O. 1997. BIONJ: an improved version of the NJ algorithm based on a simple model of sequence data. Mol Biol Evol 14:685-695.

15. Carver TJ, Rutherford KM, Berriman M, Rajandream M-A, Barrell BG, Parkhill J. 
2005. ACT: the Artemis comparison tool. Bioinformatics 21:3422-3423.

16. Beare PA, Unsworth N, Andoh M, Voth DE, Omsland A, Gilk SD, Williams KP, Sobral BW, Kupko JJ, Porcella SF, Samuel JE, Heinzen RA. 2009. Comparative Genomics Reveal Extensive Transposon-Mediated Genomic Plasticity and Diversity among Potential Effector Proteins within the Genus Coxiella. Infect Immun 77:642-656. 



\section{Chapter $\mathbf{5}$}

\section{Genome plasticity and polymorphisms in critical genes correlate with increased virulence of Dutch outbreak-related Coxiella burnetii strains}

Runa Kuley, Eric Kuijt, Mari A. Smits, Hendrik I. J. Roest, Hilde E. Smith and Alex Bossers 


\begin{abstract}
Coxiella burnetii is an obligate intracellular bacterium and the etiological agent of $\mathrm{Q}$ fever. During 2007-2010 the largest Q fever outbreak ever reported occurred in The Netherlands. It is anticipated that strains from this outbreak demonstrated an increased zoonotic potential as more than 40,000 individuals were assumed to be infected. The acquisition of novel genetic factors by these C. burnetii outbreak strains, such as virulence-related genes, has frequently been proposed and discussed, but is not proved yet. In the present study, the whole genome sequence of several Dutch strains (CbNL01 and CbNL12 genotypes), a few additionally selected strains from different geographical locations and publicly available genome sequences were used for a comparative bioinformatics approach. The study focusses on the identification of specific genetic differences in the outbreak related CbNL01 strains compared to other $C$. burnetii strains. In this approach we investigated the phylogenetic relationship and genomic aspects of virulence and host specificity. Phylogenetic clustering of whole genome sequences showed a genotype-specific clustering that correlated well with the clustering observed using Multiple Locus Variable number Tandem Repeat Analysis (MLVA). Ortholog analysis on predicted genes and single nucleotide polymorphism (SNP) analysis of complete genome sequences demonstrated the presence of genotype-specific gene contents and SNP variations in the various $C$. burnetii strains. It also demonstrated that the currently used MLVA genotyping methods are highly discriminatory for the investigated outbreak strains. In the fully reconstructed genome sequence of the Dutch outbreak NL3262 strain of the CbNL01 genotype, a relatively large number of transposonlinked genes were identified as compared to the other published complete genome sequences of $C$. burnetii. Additionally, large numbers of SNPs in its membrane proteins and predicted virulenceassociated genes were identified in all Dutch outbreak strains compared to the NM reference strain and other strains of the CbNL12 genotype. The presence of large numbers of transposable elements and mutated genes, thereof most likely resulted in a high level of genome rearrangements and a genotypespecific pathogenicity of outbreak strains. Thus, the epidemic potential of Dutch outbreak strains could be linked to increased genome plasticity and mutations in critical genes involved in virulence and the evasion of the host immune system.
\end{abstract}




\section{Introduction}

Coxiella burnetii is the pathogenic agent of $\mathrm{Q}$ fever which is a zoonotic infectious disease [1]. It is an obligate intracellular gram-negative bacterium, which thrives within the acidic parasitophorous vacuole (PV) of eukaryotic cells [2]. Domestic ruminants such as goats, sheep and cattle are usually the primary reservoir for the C. burnetii strains causing Q fever in humans [3]. The main clinical symptoms of $\mathrm{Q}$ fever widely differ between hosts $[1,3,4]$. Abortions are usually manifested in goats $[5,6]$, whereas clinical symptoms are rarely observed in cattle $[7,8]$. During abortions in goats and parturition of infected does, a large number of bacteria are excreted by infected animals into the environment $[9,10]$. Inhalation of pathogen-contaminated aerosols is the main route of infection in humans [9]. Most of the infected human individuals ( $60 \%)$ remain asymptomatic after exposure to the pathogen. In symptomatic patients acute infections are usually presented as flu-like illness and pneumonia. Around $1-5 \%$ of these cases can develop into chronic infections often leading to lifethreatening endocarditis $[1,8,11,12]$.

Q fever is prevalent throughout the world, but during 2007-2010 an unprecedented Q fever outbreak occurred in The Netherlands and is referred to as the largest outbreak ever reported $[11,13-$ 15]. During this epidemic, several isolates from different host species were isolated and cultured. Genotyping by Multiple Locus Variable-number Tandem Repeat Analysis (MLVA) revealed the predominant presence of the so-called CbNL01 genotype and, to a lesser extent, the CbNL12 genotype among the strains isolated from The Netherlands [6]. The CbNL01 genotype was identified mostly in strains isolated from goats and specifically from human patients, indicating that goats harboring the CbNL01 genotype strain were most likely the source of the large human Q fever outbreak in The Netherlands. These genotyping studies confirmed the earlier observed epidemiological link between an increase in human $\mathrm{Q}$ fever cases and a high abortion rate in goats $[6,13,15-18]$. Additionally, the genotyping studies also showed that the CbNL12 genotype was majorly associated with strains originating from cattle and rarely with strains from goat, sheep and humans $[6,13,19,20]$.

As an intracellular pathogen, the most important factor for natural selection of C. burnetii could be the interactions with its specific host niches. Genome reduction and the presence of mobile genetic elements and virulence related pseudo-genes throughout the genome are predicted to be specific genome manifestations of the obligate intracellular lifestyle of this pathogen [21]. Hence, a comparison of $C$. burnetii genome sequences with specific emphasize on genes involved in pathogenhost (cell) interactions or modulation thereof, may shed light on adaptation mechanisms of C. burnetii to various host species. Currently lipopolysaccharide (LPS) is the only biologically validated virulence factor of C. burnetii and relatively little is known regarding other potential virulence factors, or factors involved in pathogen-host interactions and host specificity [21-23]. Previous studies showed the upregulation of several genes in a Dutch outbreak strain (602) under in vivo conditions as compared to in vitro cell-based and cell-free growth conditions. These studies suggested a role of these up-regulated genes during intracellular survival and replication of $C$. burnetii in hosts. Unfortunately, a large 
number of the identified in vivo up-regulated genes were of unknown function limiting the information derived from this study with regard to adaptation processes of the bacterium in hosts [23].

C. burnetii strains with a different genotypic profile can infect a variable range of host species with a different efficiency. For example; the CbNL01 genotype strains are predominantly found in goats and in humans, whereas the CbNL12 genotype strains are commonly found in cattle and hardly in goats and humans $[6,19,20,24]$. This suggests a higher susceptibility of humans and goats to $C$. burnetii of the CbNL01 genotype than to the CbNL12 genotype strains. Identification of the genomic variations between different genotypic strains by comparative analysis using bioinformatics approaches may help in the identification of the genetic factors of $C$. burnetii involved in its host and cell tropism. Till now this has not been assessed yet with the Dutch outbreak strains. Therefore, in the present study, we sequenced and compared whole genome sequences of several strains with different genotypic profiles and originating from various hosts to assess host-specific and/or genotype-specific genetic signatures of $C$. burnetii. Main focus during this study was on strains of the CbNL01 genotype which were predominantly found in The Netherlands during the large outbreak, in order to identify genomic signatures associated with their hyper-virulence behavior. From the performed research, we conclude that an increased genome plasticity and an increased number of single nucleotide polymorphisms in genes, potentially involved in virulence and the evasion of bacterial recognition by the host immune system, is linked to the increased epidemic potential of the Dutch outbreak strains.

\section{Materials and methods}

\section{Bacterial strains and isolation of genomic DNA}

A summary of the features of all the C. burnetii strains used in this study is indicated in Table 1 . The strains from the Netherlands were primarily isolated from aborted placentas of goats, sheep and from heart valves of human chronic infected patients during the Q fever outbreak period (2007-2010) [6]. This study also involves strains isolated from aborted placentas of goats and cattle from France. Additionally, a few strains were isolated from Q fever infected humans from elsewhere in the world. All the strains were genotyped based on Multiple Locus Variable-number Tandem Repeat Analysis (MLVA) [6]. The majority of the strains used for sequencing were cultured with a minimal number of sub-passages in BGM cells [10]. A few strains were cultured axenically in acidified citrate cysteine medium (ACCM-2) also maintaining a minimum number of sub-passages to prevent phase variation (Table 1) [25]. Genomic DNA was isolated using the phenol-chloroform method [26] after overnight incubation with ATL lysis buffer and proteinase K (QIAGEN, Hilden, Germany). A prior DNase treatment was performed to the bacterial pellet of cell-culture cultivated strains to eliminate most of the host derived DNA. 


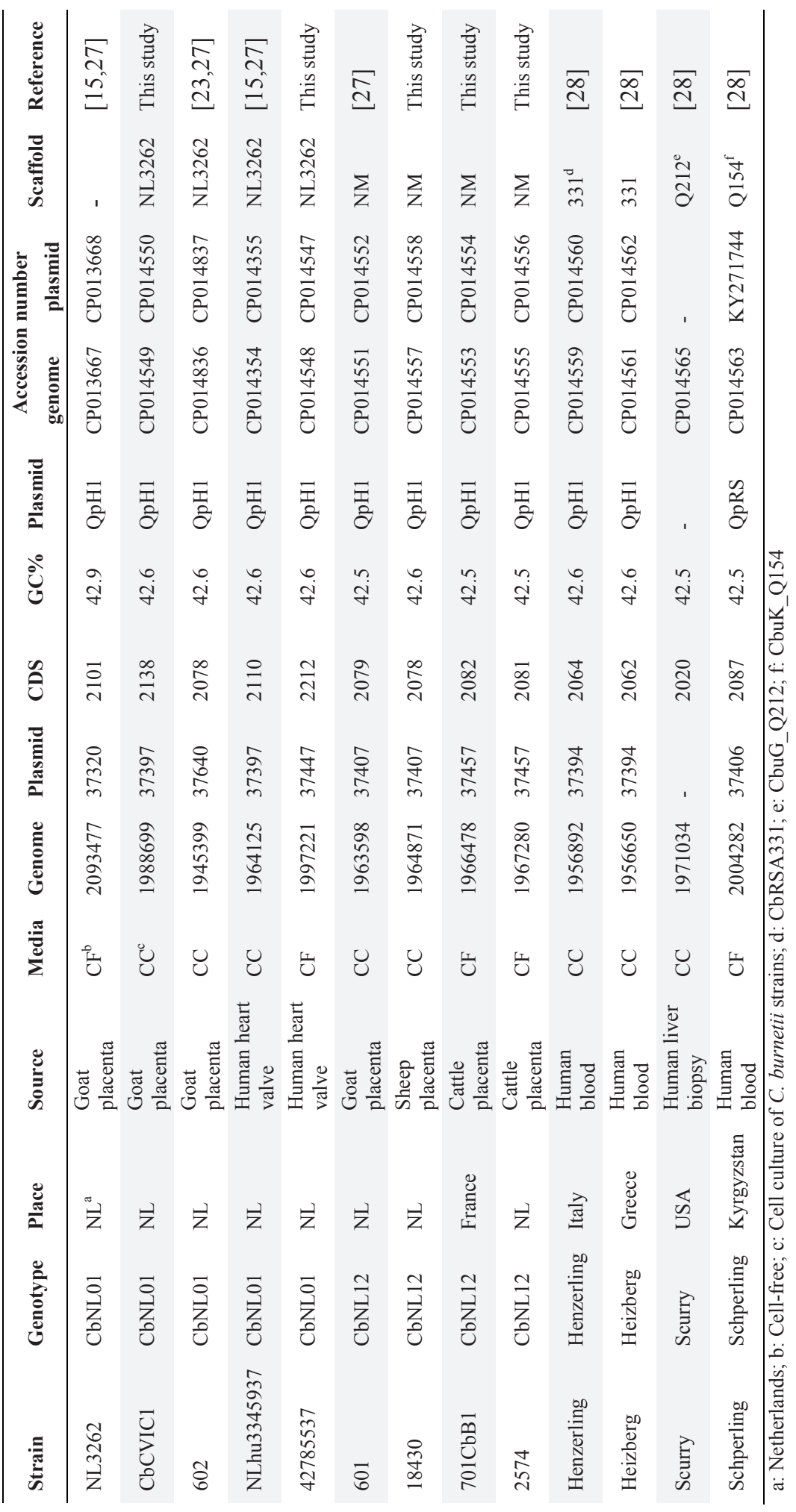




\section{Genome sequences of $C$. burnetii}

Draft genome sequences of all C. burnetii strains (except 602) were de novo reconstructed from Illumina MiSeq paired-end $250 \mathrm{bp}$ reads using SPAdes-3.6.2 [29]. The draft genome sequence of the 602 strain was obtained by using Roche 454XL sequencing technology and was de novo assembled by Newbler 2.6. The Dutch outbreak representative strain NL3262 was fully reconstructed using a combination of sequencing technologies (Illumina MiSeq paired-end $250 \mathrm{bp}$, PacBio RS and Roche 454XL) as described previously [15]. The contigs of the draft genome sequences were scaffolded and joined into an artificial chromosome based on homology with the closest $C$. burnetii strain from the database using BLAT synteny tool (Table 1) [30]. Mapping the reads back on the draft genome sequences using Bowtie2 aligner [31] confirmed its correct synteny and occasionally helped to close minor gaps. All C. burnetii (complete and draft) genome sequences were annotated by the NCBI Prokaryotic Genome Annotation Pipeline (http://www.ncbi.nlm.nih.gov/genome/annotation_prok).

Table 2. Genome sequences of $C$. burnetii strains obtained from NCBI database for phylogenetic analysis and their in silico MLVA genotype

\begin{tabular}{|c|c|c|c|c|c|c|}
\hline Strain & Accession No. & Source & MLVA & Genome & Plasmid & Reference \\
\hline NMRSA493 $^{\mathrm{c}}$ & NC_002971.3 & Tick & NM & 1995281 & QpH1 & {$[21]$} \\
\hline $\mathrm{Cb} 175^{\mathrm{c}}$ & HG825990.3 & Human & NM & 1989565 & QpH1 & [32] \\
\hline $\mathrm{Cb} 185^{\mathrm{d}}$ & NZ_CBTH000000000.1 & - & NM & 1991515 & unknown & - \\
\hline $\mathrm{Z} 3055^{\mathrm{c}}$ & PRJEB1438 & Sheep & $*$ & 1995463 & QpH1 & [33] \\
\hline $\mathrm{Cb} 109^{\mathrm{d}}$ & AKYP00000000 & Human & CbNL01 & 2030000 & QpH1 & [34] \\
\hline $\mathrm{EV}-\mathrm{Cb} \_\mathrm{C} 13^{\mathrm{d}}$ & CCAM 01000000 & Ruminant & CbNL01 & 2023172 & unknown & [35] \\
\hline NL-Limburg ${ }^{\mathrm{d}}$ & JZWL00000000 & Human & CbNL01 & 2214254 & QpH1 & [14] \\
\hline $\mathrm{Cb}-\mathrm{B} 1^{\mathrm{d}}$ & ССAH01000000 & Ruminant & CbNL12 & 2008014 & QpH1 & [35] \\
\hline EV-Cb_BK $10^{\mathrm{d}}$ & CCAL01000000 & Ruminant & CbNL12 & 1999727 & unknown & [35] \\
\hline $\mathrm{Cb} \_\mathrm{B} 18^{\mathrm{d}}$ & CCAI01000000 & Ruminant & CbNL12 & 2008445 & unknown & [35] \\
\hline CbRSA $331^{\mathrm{c}}$ & NC_010117.1 & Human & CbRSA331 & 2016427 & QpH1 & - \\
\hline Dugway $^{\mathrm{c}}$ & NC_009727.1 & Rodents & Dugway & 2158758 & QpDG & {$[21]$} \\
\hline CbuG_Q212 ${ }^{\mathrm{c}}$ & NC_011527.1 & Human & CbuG_Q212 & 2008870 & integrated & [21] \\
\hline CbuK_Q154' & NC_011528.1 & Human & CbuK_Q154 & 2063100 & QpRS & [21] \\
\hline $\mathrm{Q} 321^{\mathrm{d}}$ & AAYJ01000000 & Cattle & Q321 & 2004584 & QpDV & {$[36]$} \\
\hline Goat Q177 ${ }^{\mathrm{d}}$ & NZ_AAUP00000000.2 & Goat & Goat Q177 & 2090565 & QpRS & - \\
\hline $\mathrm{Cb} \_\mathrm{O} 184^{\mathrm{d}}$ & CCAK01000000 & Ruminant & Cb_O184 & 2168222 & QpRS & [35] \\
\hline Namibia $^{\mathrm{d}}$ & СР007555 & Goat & Namibia & 2101438 & QpRS & [37] \\
\hline $\mathrm{AuQ} 01^{\mathrm{d}}$ & JPVV00000000 & Human & AuQ01 & 2073000 & QpRS & [38] \\
\hline \multicolumn{7}{|c|}{$\begin{array}{l}\text { c: complete genome sequence (Genome in bp) } \\
\text { d: draft genome sequence (Genome in bp) } \\
\text { *: in silico MLVA genotype more similar to NM genoty }\end{array}$} \\
\hline
\end{tabular}


The (draft) genome sequences and plasmids of all sequenced C. burnetii strains have been deposited at GenBank under accession numbers indicated in Table 1. Published genomic sequences of several other C. burnetii strains used for phylogenetic studies were obtained from GenBank (Table 2).

\section{Genotyping of $C$. burnetii strains}

All the sequenced strains were genotyped by MLVA using a selection of 10-12 loci described previously [6,39]. As an additional verification step, genotypes of the reconstructed genome sequences as well as published genome sequences of strains from NCBI database were checked by in silico MLVA genotype analysis using isPCR (BLAT), MLVA primers and genotype references. In silico genotypes of all C. burnetii sequenced strains corresponded with known/measured MLVA genotypes. The in silico analysis of the database strains gave us information on their MLVA genotype (Table 2). Based on the number of repeats per genome loci a minimum spanning tree method was used to cluster the MLVA genotypes using Bionumerics (version 6.6) with default settings.

\section{Sequence clustering and phylogenetics}

Reconstructed as well as database obtained C. burnetii sequences were hierarchical clustered based on their MUMi distance [40] and displayed as a phenogram using the BioNJ algorithm [41]. The underlying MUMi distance matrix was calculated from the pair-wise non-overlapping maximal unique matches (MUMs; using Nucmer version 3.22) [42]. Relative pair-wise distances (MUMi) were obtained by dividing the sum of pair-wise MUMs by the average genome size of the two compared genomes. This MUMi distance varies between 0 to 1 where 0 indicates very similar genomes and 1 is for very distant genomes. MUMi trees were visualized in SplitsTree4 [43].

For SNP detection in the sequenced draft genomes and in the complete genome of strain NL3262, reads with low quality (bases with a quality score of $\leq$ Q20) were removed before SNP calling. High quality reads were used to map against reference NM genome (GenBank: NC_002971.3) using Bowtie2 short-read aligner [31]. SNP calling was performed using bcftools [44] using default parameters. SNP sequence alignments were made for only those positions in NM reference strain where each of the sequenced strain had sequence coverage. Phylogenetic trees based on SNPs were created by Clustal Omega (https://www.ebi.ac.uk/Tools/msa/clustalo/) using standard settings [45] and visualized in SplitsTree4 [43]. SNPs and insertions/deletions in CbNL01 and CbNL12 strains compared to NM were verified at single nucleotide level of the mapped reads.

\section{Comparison of $C$. burnetii genome contents}

Alignment of the complete genome sequence of strain NL3262 with the sequences of the reference NM, and strains which were related to the outbreak CbNL01 genotype (Z3055, NL-Limburg) [14,33] was performed by using Mauve 2.4.0 aligner software with default parameters [46]. Protein orthologs analysis of C. burnetii amino acid sequences obtained from GenBank files from NCBI were performed 
using Proteinortho software (Version 5) with default settings [47]. Orthologs and unique proteins in sequenced strains were identified from the orthologous list generated by Proteinortho. Additionally, ortholog analysis of sequenced strains was conducted using the proteome of NM strain as a reference. Pseudo-genes were excluded from the ortholog analysis. Orthologs from different strains were plotted by means of Edwards-Venn diagrams using Venerable package (Version 2.0). For a more detailed genomic sequence comparison, Artemis Comparison Tool (ACT) [48] was used to visualize whole genome MUMmer alignments. Detailed analyses were performed on virulence related genes such as variants (mutations/ deletions) in LPS encoding region, which were performed by comparing $C$. burnetii genome sequences to LPS encoding region of NM-I (GenBank: AF387640). Also, variants found in the CbNL01 and CbNL12 strains were subsequently pairwise checked against the reference NM strain using ACT and ClustalW [49]. We also performed a COG (Cluster of Orthologous Groups) enrichment-analysis on all non-synonymous genes in CbNL01 and CbNL12 strains and analyzed the proportions of different proteins for each COG category.

\section{Results}

\section{Genome sequences of $C$. burnetii strains}

In the present study we report the new genome sequences of 11 C. burnetii strains. The reconstruction of the genome sequences of the NL3262 and NLhu3345937 strains was described before [15]. Table 1 is a summary of the main features of the sequenced genomes. Except for strain NL3262, all the other strains are reported as draft genome sequences. The assembled contigs of these sequenced strains are joined into artificial chromosomes based on scaffolding against the closest genome sequence present in the database. In C. burnetii genome sequences, the insertion sequence (IS) elements are dispersed all over the chromosome and are not found on the plasmid [50]. These elements are repetitive in nature and as a consequence, the majority of contig-breaks in the draft genome assemblies mapped to the positions of an IS sequence, when analyzed against the NM reference genome sequence (GenBank: NC_002971.3). As expected, all C. burnetii sequenced genomes possess a single circular chromosome of around $2 \mathrm{Mb}$ and a QpH1 plasmid of $37 \mathrm{~kb}$ size (except the Schperling and Scurry strains). The bacterial genomes of all sequenced strains are predicted to contain between 1800-2062 coding sequences (CDSs). Around 35-39\% of these CDSs were annotated as encoding hypothetical products based on the NCBI Prokaryotic Genome Annotation Pipeline. In silico MLVA typing of the genome sequences corresponded with similar profiles of strains as measured by lab-based MLVA measurements (Table 2).

\section{Genotype-specific structural similarities between $C$. burnetii genome sequences}

Whole (complete and draft) genome-based phylogenetic analysis was used to infer the relationships between sequenced $C$. burnetii strains of different genotypes. The strains were initially isolated from different hosts such as goats, sheep, cattle and human patients (both acute and chronically infected) 
(Table 1). The analysis also included whole genome sequences of C. burnetii strains that have become available in the NCBI database (Table 2). Genome sequences of some of the strains from the database, present as draft sequences (several contigs), were scaffolded similarly to our reconstructed genomes and joined into artificial chromosomes based on the reference NM strain. The phylogenetic analysis of these genome sequences were based on MUMi distance. This approach takes into account the number of maximum unique and exact matches (MUMs) of a given minimal length shared by the two genome sequences being compared [40,42]. The phylogenetic analysis was conducted for genomes excluding the plasmids, as presence or absence of plasmids did not result in any difference in hierarchical clustering of C. burnetii strains.

Based on the clustering analysis, the strains could be divided into 4 main clades (indicated as 1a, 1b, 2 and 3 in Fig.1). The majority of the C. burnetii sequenced strains segregated into two clusters (cluster 1 ( $\mathrm{a}$ and $\mathrm{b}$ ) and cluster 2, Fig. 1). These two clusters correspond to the two major genotypes (CbNL01 and CbNL12) of strains isolated from The Netherlands, respectively. Strain NL3262 and the database strain NL-Limburg clustered closely together (Cluster 1a, Fig 1). The genome of strain NL3262 is a complete reconstructed genome derived and curated using different sequencing technologies [15]. The NL-Limburg genome is a draft genome generated with a single sequencing technology (PacBio) and its assembly constitutes 4 contigs representing the chromosome [14]. The contigs of NL-Limburg cover the complete genome of NL3262 without any gaps (as visualized by ACT; data not shown). The ends of the contigs in NL-Limburg contain overlapping sequences of around 50,000 bp. Due to these additional sequences (which are probably the result of assembly error) the genome of strain NL-Limburg (represented as ** in Fig 1) is clustered at some distance away from strain NL3262 (MUMi distance 0.025). Upon removal of these repetitive sequences the MUMi distance between strains NL-Limburg (indicated in cluster 1a without **) and NL3262 decreases to 0.01 , indicating nearly identical genomes with differences based on 8 SNPs. Draft genome sequences of the database strains (Cb109 and EV-Cb_C13) belonging to the CbNL01 genotype (Fig 2 and Table 2) clustered with the CbNL01 strains $(602$, CbCVIC1, 3345937, 42785537) originating from The Netherlands in cluster 1b (Fig 1). Strains NL3262 and NL-Limburg belong to the CbNL01 genotype strains as well (Fig 2 and Table 2) [14,15]. The major differences between the complete and draft genome sequences of the strains belonging to the clusters $1 \mathrm{a}$ and $1 \mathrm{~b}$ are the genomic locations of highly repetitive transposon sequences. The genome sequence of strains NL3262 and NL-Limburg contain 109 and 115 IS elements respectively scattered around the chromosome. These IS elements are missing in draft genome sequences, most probably due to their presence at contig-break regions, as indicated above. Thus, due to these structural differences, the draft genome sequences cluster very closely to each other (Cluster 1b) relative to the complete genome sequences (Cluster 1a). The complete sequence of NL3262 and the nearly complete genome sequence of NL-Limburg (belonging to genotype CbNL01) differ with a MUMi distance of around 0.05-0.06 from the draft genome sequences in cluster $1 \mathrm{~b}$ which also belong to CbNL01 genotype. 
Cluster 2 includes the draft genome sequences of strains Henzerling, Heizberg and the sequences of the CbNL12 genotype group of strains 601, 701CbB1, 2574 and 18430 (Fig 1). Apart from these, cluster 2 contains the database strains belonging to the CbNL12 genotype (draft genome sequences: CbB1, Cb_B18, EV-Cb_BK10) as well as the reference NM like genotype strains (NM, CbRSA331, Cb175, Cb185) (Fig 2 and Table 2). The complete genome sequence of strain Z3055 was placed at an equidistance (MUMi distance 0.02) from clusters 1 (CbNL01) and 2 (CbNL12) (Fig 1). An in silico PCR analysis of strain Z3055 showed few MLVA markers similar to CbNL01 genotype but the majority indicative for the NM genotype. This resulted in the presence of Z3055 in-between the nodes of the NM and CbNL01 genotype strains in an MST analysis (Fig 2). Based on the MST and

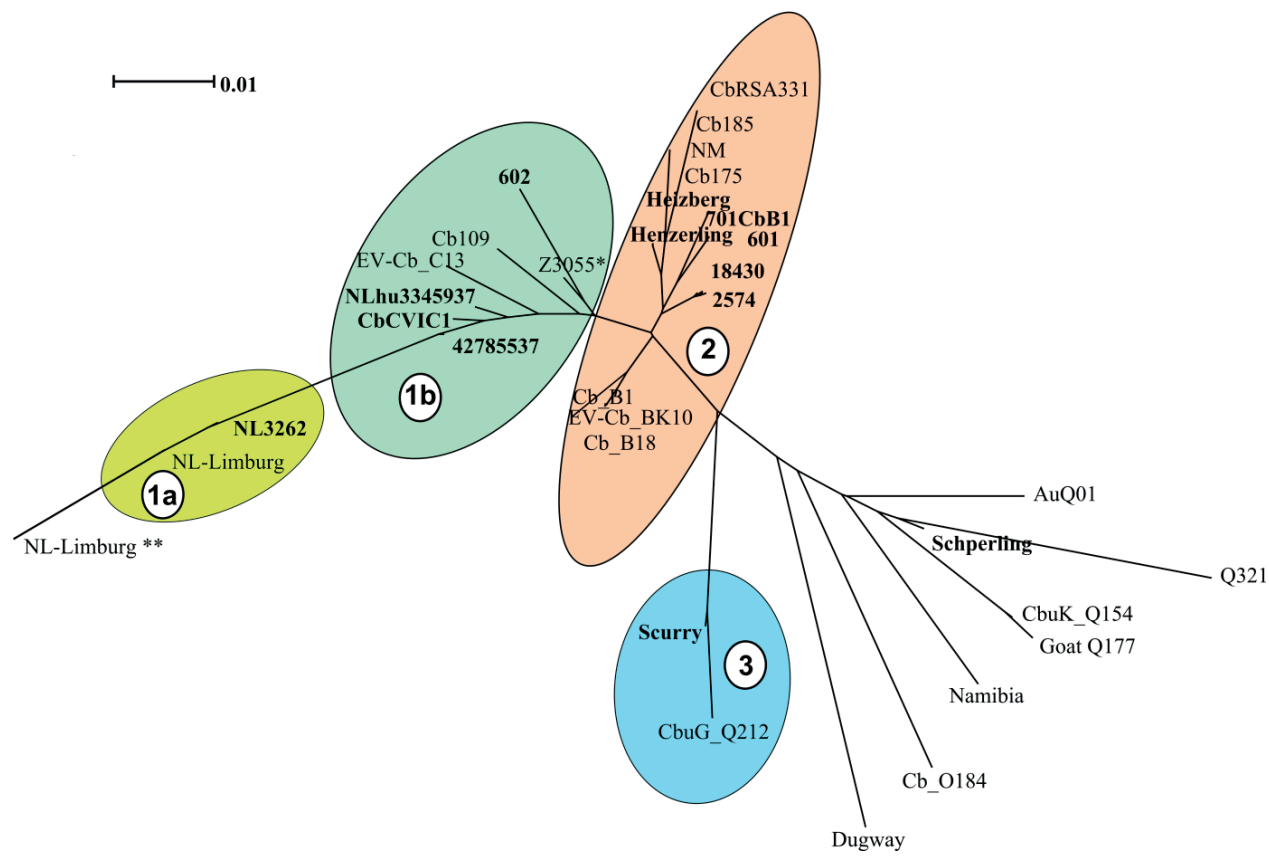

Figure 1. Phylogenetic relationships among C. burnetii genome sequences. C. burnetii whole (complete and draft) genomes from newly sequenced strains and strains obtained from NCBI database were hierarchical clustered and are displayed as a phenogram using the BioNJ algorithm. Strains in bold font are newly sequenced strains and other strains are genome sequences obtained from NCBI database. Scale bar indicates $1 \%$ of genome variability. Clades representing strains from the same MLVA genotype are indicated by colored clouds. Clades contain strains with distance ranging from 0.010 to 0.021. Clade 1a and 1b: CbNL01, Clade 2: CbNL12 and NM like genotype, Clade 3: Scurry genotype (Plasmid-less strains), non-clustered strains are each of different genotypes (except for the sequenced Schperling strain and NCBI obtained Q321 strain, which are of same genotype).

* represents the genome of strain Z3055 which is equidistant from cluster 1 and 2,** represent the genome of strain NL-Limburg with duplicated regions. 
phylogenome analysis, we assessed the genome sequences of strains Z3055 and NM to be very closely related. Cluster 3 included strain Scurry and the database strain CbuG_Q212, both belonging to the same genotype (Figs. 1 and 2). These strains clustered separately as they contain plasmid-homologous regions integrated in their genomes unlike other strains (Fig 1, Table 1) [51]. From the phylogenetic analysis, the Schperling strain was found to be the most distant strain as compared to the other de novo sequenced strains used in this study (MUMi distance 0.05) (Fig.1). The genome sequences of strains Q321, CbuK_Q154 and Goat Q177 were most similar to the strain Schperling with a MUMi distance of around 0.025-0.03. However, except for strain Q321, these strains belonged to different genotypes (Fig 2). Hence, a phylogenetic clustering of the strains based on an MLVA classification rather than on the host-origin background indicated a high-level of genome sequence similarity of genotypespecific C. burnetii strains.
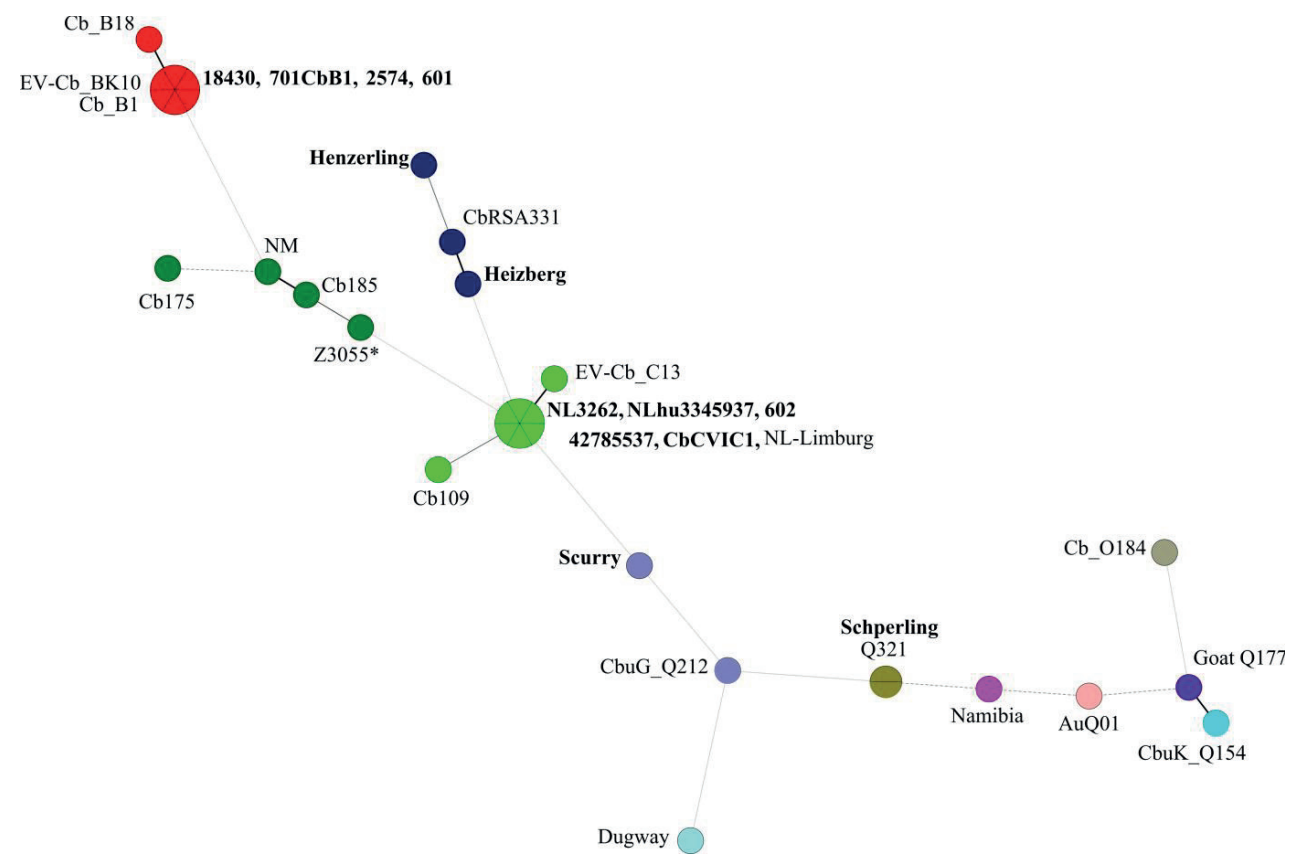

Figure 2. Minimum spanning tree (MST) analysis of the in silico MLVA genotypes from C. burnetii strains used in this study and sequences obtained from NCBI database. A total of 12 to 17 micro satellite loci (depending on coverage in each particular genome sequence) were used to construct a dendogram using Bionumerics (version 6.6). Each circle represents a strain and the size of the circle corresponds to the number of strains with the same genotype. Colors indicate the genotype of the strain. The connecting lines refer to the distance, while the intensity of lines shows closeness between the strains. Strains in bold font are newly sequenced strains and other strains are sequences obtained from NCBI database.

* represents the in silico MLVA genotype of strain Z3055 (the majority of the MLVA markers are of the NM genotype and a few of the CbNL01 genotype). 
A phylogenetic tree was also reconstructed using the identified SNPs among the sequenced strains with respect to the reference strain NM using bcftools (Fig 3) [44]. The SNP tree was constructed using Clustal Omega [45]. At SNP level, strains belonging to similar genotypes clustered together with clade 1 containing the CbNL01 genotype strains; clade 2 containing the strains Henzerling and Heizberg and clade 3 containing the CbNL12 genotype strains. This indicates that the presence of similar SNPs in the genomes of the same genotype strains relative to the reference strain NM. Strains Schperling and Scurry clustered separately, indicating that these strains contain SNPs distinct from the SNPs present in other sequenced strains compared to the reference strain NM. The data corresponds to the presence of distinct genotypes of Schperling and Scurry compared to other sequenced strains (Fig 2). This SNP based clustering of genotype-specific strains further confirms the highly similar genome sequences of strains belonging to the same genotype.

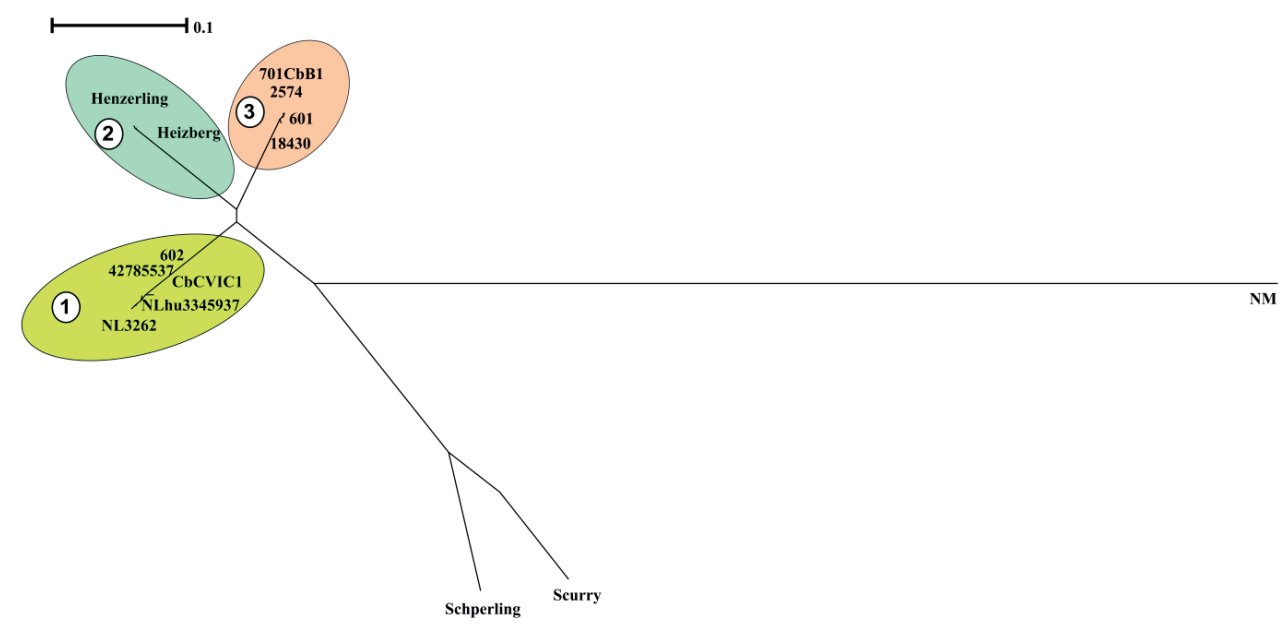

Figure 3. Phylogenetic relationships among sequenced C. burnetii genomes based on SNP analysis. The phylogenetic tree is based on SNPs among genomes of the sequenced strains with respect to the genome of reference strain NM using bcf tools. The SNP tree was constructed using Clustal Omega and the clustering is displayed as a phenogram using the BioNJ algorithm. The scale bar indicates the number of substitutions per site. Clades representing strains from the same genotype are indicated by colored clouds. Clade 1:CbNL01, Clade 2: Henzerling and Heizberg (similar genotype), Clade 3: CbNL12. The Schperling and Scurry strains are present separately due to the presence of distinct SNPs relative to other sequenced strain genomes. NM indicates the polymorphic regions in sequenced strain genomes relative to the NM reference genome.

\section{Conserved and unique gene content in $C$. burnetii sequenced genomes}

A comparison of the genome sequences of $C$. burnetii shows that all sequenced strains are highly conserved in terms of size, number of coding sequences and in nucleotide composition (Table 1). The degree of conservation is illustrated by the comparison of the coding capacity of these genomes: 1447 
predicted proteins are orthologs in all sequenced 13 C. burnetii strains in this study (with a total CDS count ranging from 1800-2062) among which 1409 of the predicted proteins are orthologs with reference NM. Remaining 38 proteins not shared with NM are majorly hypothetical protein products mostly occurring due to modifications (such as point mutations, small deletions/ insertions of $<50 \mathrm{bp}$ ) in existing NM pseudo-genes (Supplementary Table 1). The 1409 orthologs among all strains constitute the coding core genome. All sequenced C. burnetii strains contain the QpH1 plasmids, except for strain Schperling containing QpRS plasmid and the plasmidless strain Scurry. The QpH1 plasmids from sequenced strains were all very similar to the reference NM QpH1 plasmid (Accession No. NC_002118.1) with differences on an average of 58 point mutations. The draft plasmid sequence of Schperling was similar to the published sequence of plasmid QpRS (Accession No. Y15898.1) with differences in 63 SNP and a gap of $1810 \mathrm{bp}$ (data not shown).

Ortholog analysis of the proteome of the sequenced C. burnetii strains showed the presence of a few unique proteins annotated as hypothetical protein in CbNL01 and CbNL12 strains (data not shown). Further analysis of genes encoding these unique proteins showed that these genes were present at the beginning or end of contigs in assembled draft genome sequences. As contig ends of draft genome sequences are highly repetitive, genes annotated in these regions were not considered as unique genes, because these genes were truncated transposase genes. Taking this factor into account and other manual annotation corrections, we found no unique complete genes encoded in CbNL01, CbNL12, Henzerling and Heizberg strains. Instead of unique genes for each strain we did find orthologs that were genotype-specific. These orthologs were shared between strains of the same genotype only and were annotated as hypothetical proteins. When we compared these genotypespecific genes at the nucleotide level to the genome of the reference strain NM, mutations or partial ORF deletion in existing NM pseudo-genes were observed for which altered functionality or regulation cannot be directly inferred (Supplementary Table 2).

By comparing the proteome of the 13 sequenced strains (Table 1) 50 and 98 unique protein encoding genes were identified in strains Scurry and Schperling, respectively. Out of these, 3 and 17 genes are exclusively found in Scurry and Schperling, whereas the other genes were orthologs with the closely related strains CbuG_Q212 and CbuK_Q154, respectively (Fig 1). These unique genes in Scurry and Schperling strains were annotated as hypothetical proteins and result from point mutations in existing pseudo-genes of CbuG_Q212 and CbuG_Q154 (data not shown).

\section{Highly similar C. burnetii gene content within the same genotype-groups}

A comparison of the coding capacity of the genomes of strains belonging to the same genotype showed $98 \%, 99.4 \%$ and $100 \%$ orthologs in predicted proteins of CbNL01, CbNL12 and the human strains (same genotype Henzerling and Heizberg strains), respectively (Fig 4). Fifty-one unique genes (non-orthologs) of NL3262 encode for transposase associated proteins (Fig 4a). These genes are not properly annotated in 602 , CbCVIC1, NLhu3345937 and 42785537, because they fall within contig- 
break regions of the draft genome sequences. The high level of similarities of the proteomes between these strains indicates that the gene content is highly conserved within strains of the same genotype.

a.

b.
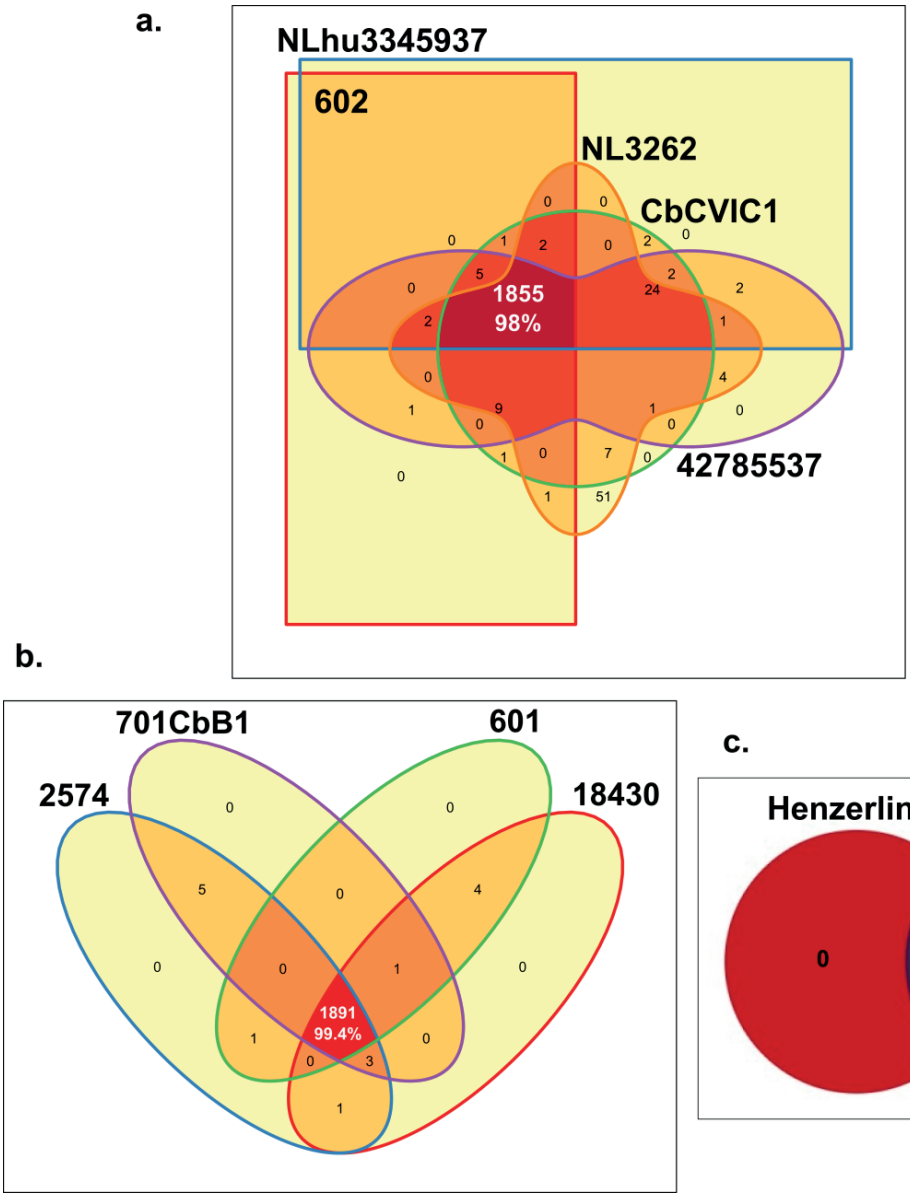

c.

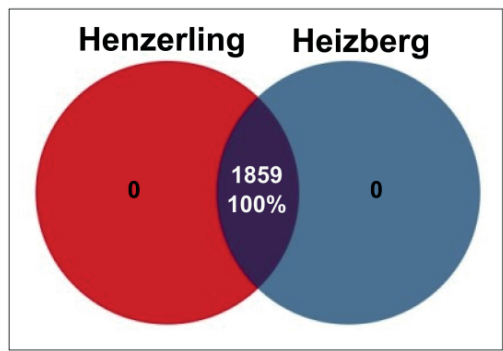

Figure 4. Edwards-Venn diagrams showing the number of orthologs and unique predicted coding proteins among strains of the same MLVA genotype. Number of ortholog genes shared between a) CbNL01 genotype strains b) CbNL12 genotype strains and c) human strains Henzerling and Heizberg is represented by EdwardsVenn diagrams. Transposase coding proteins were included in the analysis and pseudogenes were scored as absent.

As all the 13 C. burnetii strains included in this study were initially isolated from different hosts, a comparison was made to assess the percentage of proteins shared by strains derived from each host-species. These comparisons resulted in the presence of $94.6 \%, 100 \%, 87.9 \%$ and $87 \%$ orthologs in predicted proteins of goat, cattle, human-acute and human-chronic isolated strains respectively (Supplementary Fig 1). The lower percentage of orthologs shared by strains obtained from various 
host species is due to the presence of different genotypes within each host-species, except for the cattle-derived strains, as both cattle strains were of same CbNL12 genotype. For example; the strains isolated from goat include both CbNL01 (NL3262, 602, CbCVIC1) and CbNL12 (601) genotypes (Supplementary Fig 1b). Further, the strains isolated from acute and chronic human Q fever patients contain more divergent strains (Schperling and Scurry respectively). Because of this, the protein orthologs of acute and chronic human strains are comparatively less ( $~ 87 \%$, Supplementary Fig $1 \mathrm{c}$ and 1d). Taken together, the percentage of orthologs in strains obtained from similar host-species is less conserved than the strains obtained from similar genotype. This clearly indicates the presence of genotype-specific functional characteristics of $C$. burnetii strains.

\section{Architecture of the NL3262 complete genome sequence}

To assess the genome architecture of strain NL3262 and to study its putative genome rearrangements, the complete genome sequence of strain NL3262 was aligned with the genome sequence of strains NM, Z3055 and NL-Limburg using the Mauve genome aligner (Fig 5). Among the different $C$. burnetii strains, the CbNL01 genotype strains NL3262 and NL-Limburg encode the highest number of transposase genes (106-112 IS110 family transposase, 3 ISAs1 family transposase and 12-13 remnant transposase genes, respectively). Other completely sequenced $C$. burnetii genomes generally contain only 30-60 transposase genes $[21,33]$. Compared to the genome sequence of NL3262, chromosomal rearrangements in strains NL-Limburg, NM and Z3055 strains have resulted in 2, 19 and 21 Locally Collinear Blocks (LCBs) of the same gene content and gene order respectively (Fig 5). Strains NM and Z3055 share almost all LCBs indicating a similar genome structure (Supplementary Table 3). Relative to the genome sequences of strains $\mathrm{NM}$ and Z3055, at least 18 and 20 genome rearrangements occurred in strain NL3262, respectively. Taken together, the LCBs of strains NM and Z3055 represent 40 genomic breakpoints relative to the genome of strain NL3262 (Fig 5b and 5c). In the genome sequence of strain NL3262, 38 (95\%) of the breakpoints occurred at intact or a remnant transposase sequences of the IS110 family (Supplementary Table 3). The presence of transposase genes at almost all genome breakpoints suggests an important role of homologous recombination in order to establish the observed genome rearrangements of the Dutch outbreak strains. Unlike strains NM and Z3055, the gene order in strains NL3262 and NL-Limburg are nearly identical, yielding only 2 LCBs. The absence of rearrangements between NL3262 and NL-Limburg, indicates a similar genome structure of these CbNL01 genotype Dutch outbreak strains isolated from goat and human patient (Fig 5a).

\section{Increased number of transposons in Dutch outbreak CbNL01 MLVA genotype strains}

Genome sequencing of a number of Dutch outbreak strains belonging to the CbNL01 MLVA genotype resulted in 132, 135 and 123 contigs for strains NLhu3345937, 42785537 and CbCVIC1, respectively. In contrast, sequencing of the CbNL12 strains 601, 2574, 18430 and 701CbB1 resulted in 43, 40, 66 
a

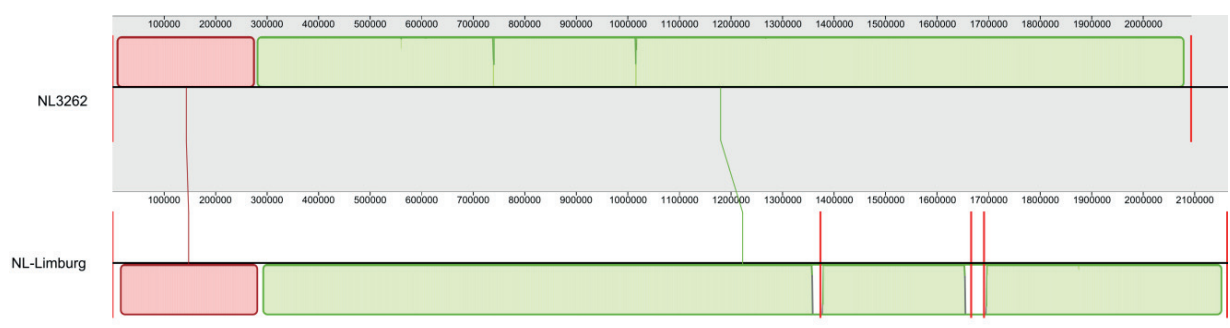

b

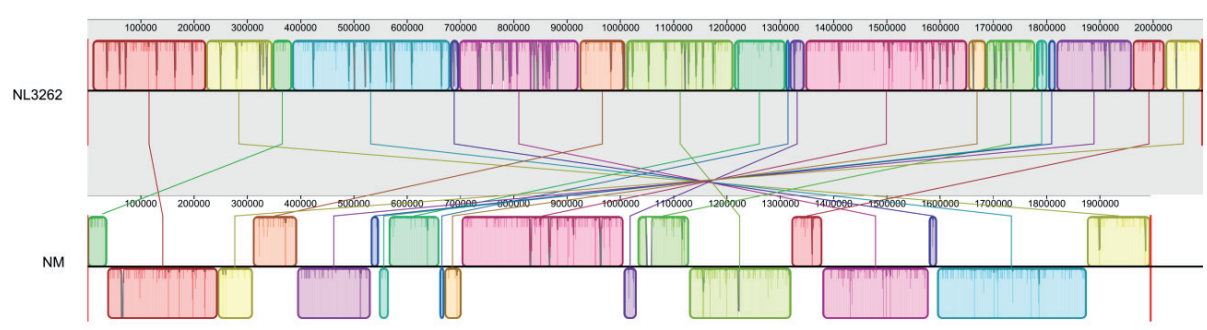

C

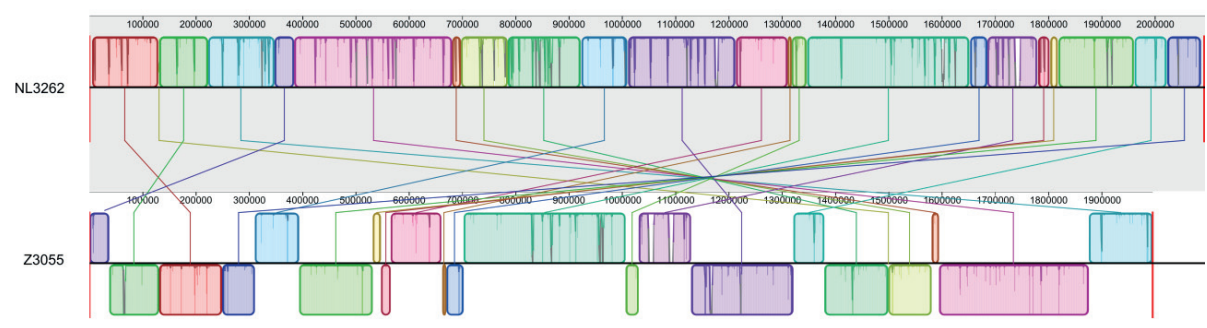

Figure 5. Alignment of the genome sequences of strains NL-Limburg, NM and Z3055 relative to NL3262. Comparisons were performed using the Mauve genome alignment tool. The genome rearrangements of strains NL-Limburg (a), NM (b) and Z3055 (c) are depicted with respect to strain NL3262. Vertical red lines for each genome indicate contig boundaries. Color-coded LCBs (Locally Collinear Blocks, regions within genome sequence that are conserved and predicted to be free of any internal rearrangements) indicate conserved segments. Upper and lower LCBs of the genomes correspond to forward and reverse orientation with respect to NL3262. Plots of sequence conservation are shown within each LCB. Regions with no color indicate no detectable homology between the two genomes. Connector lines indicate orthologous LCBs.

and 42 contigs, respectively. As described above, the majority of the contig-break points contained repeat sequences and corresponded mostly to transposase genes when mapped against the NM reference genome. Based on this observation, we anticipate that the observed number of contigs roughly corresponds to the number of transposase genes encoded by the strain. Based on this assumption, the CbNL01 and CbNL12 strains contain around 130 and 47 contigs, respectively. The assumed high numbers of transposons in the draft genome sequences of CbNL01 strains also matches with the number of annotated transposase genes in strains NL3262 (121 intact and remnant 
transposons) and NL-Limburg strains (128 intact and remnant transposons) belonging to the same genotype group. Taken together, the largest number of transposons are encoded by the CbNL01 Dutch outbreak strains as these strains contain two to three fold more transposase genes compared to other strains of C. burnetii sequenced so far [33,52].

\section{Differences in Dutch strains at SNP level}

To further asses the differences in the genome sequences of the Dutch strains from different genotypic groups, we performed a detailed NM reference-centric variant analysis. For this analysis we used the complete genome sequences of the NL3262 strain [15] and the draft genome sequence of the CbNL01 and CbNL12 strains. We found an average of 2514 SNPs between the CbNL01 strains and NM. Among these, 417 SNPs were present in intergenic regions, 1041 were synonymous SNPs in coding regions and 1056 were non-synonymous SNP which corresponded to 702 mutated genes. Between the CbNL12 strains and NM an average of 2078 SNPs were found, with 340 SNPs in intergenic regions, 889 SNPs in coding regions (synonymous SNP) and 849 non-synonymous SNPs which corresponded to 581 mutated genes. With respect to NM, these mutated genes in CbNL01 and CbNL12 genotype strains majorly encoded hypothetical proteins, membrane proteins, transporter proteins, DNA repair proteins, translation-related proteins and a few virulence-associated proteins (explained in detail below). Compared to the genome of NM, the genomes of the CbNL01 strains showed a deletion of 3600 bp (containing ankyrin-repeat containing protein; CBU_0072), a deletion of 2300 bp (containing several hypothetical proteins; CBU_0877-CBU_0880) and a larger deletion of 9700 bp (containing the peptidoglycan catabolism proteins; CBU_1101-CBU_1112). A deletion of around 2250 bp (containing several hypothetical proteins; CBU_0016-CBU_0019) was observed in the CbNL01 and CbNL12 genome sequences, relative to the NM genome sequence (Supplementary Fig 2).

With respect to mutations in membrane protein encoding genes, a total of 64 and 48 genes were mutated in the CbNL01 and CbNL12 strains out of the 104 membrane protein encoding genes in the NM strain. Thirty eight of these genes contained the same mutation in both genotype strains. Additional mutations in 26 hypothetical membrane proteins were specific for all the CbNL01 strains. Although, the exact functions of these hypothetical protein products are not known, changes in these cell surface proteins could potentially contribute to an altered antigenic profile of the CbNL01 strains. Large numbers of mutations were also seen in transporter genes, where a total of 41 and 25 genes were mutated in the CbNL01 and CbNL12 strains (from a total of 75 transporters in NM). Among these, 20 genes contained identical mutations in both genotype strains, whereas 21 genes contained mutations specific for the CbNL01 strains. These transporter genes belonged mostly to the ABC and MFS family transporters. Further COG analysis of the mutated transporter genes showed that these genes could be primarily involved in transportation of amino acid, carbohydrate and ions (COG categories E, G, P respectively). Moreover, we also identified mutations in several translation-related proteins in the outbreak CbNL01 strains. Around 29 of these mutated genes were also found in the CbNL12 strains, 
whereas 15 mutated genes were specifically found in the CbNL01 strains (from a total of 121 translation genes in NM) (Supplementary Fig 2).

Recognized virulence factors of $C$. burnetii are genes involved in primary defense mechanisms against oxidative stress. These include several DNA repair genes involved in base excision repair, nucleotide excision repair, mismatch repair, addAB mediated recombinational repair systems and oxidative stress enzymes [53]. Our variant analysis showed high number of mutations in the DNA repair genes; wherein 13 mutated genes were shared in both genotype strains and mutations in 7 genes were specific to the CbNL01 strains (from a total of 26 DNA repair genes in NM). On the contrary, we did not find any mutations in genes encoding for oxidative stress enzymes among the various strains indicating that these genes are highly conserved. The secondary defense mechanisms of C. burnetii includes the manipulation of host cell processes [53]. A Dot/Icm type IV secretion system (T4SS) is predicted to be encoded by the genome of C. burnetii homologous to the L. pneumophila Dot/Icm system. This is an important transfer system associated with the delivery of effector proteins from the bacterial cell to the host cytosol mediating PV formation and other cellular events required for bacterial maintenance and survival [23,50,54]. Among the T4SS genes, 4 genes were mutated in both genotype strains and an additional 4 genes were mutated specifically in the CbNL01 strains. Furthermore, 12 effector proteins characterized by the presence of eukaryotic like protein domains were also mutated in the CbNL01 and CbNL12 strains relative to NM. These genes are predicted to be virulence-related as the encoded proteins contain eukaryotic-like domains which may mimic host proteins and module host responses required for successful bacterial infection (Supplementary Fig 2). Taken together, compared to the reference strain NM, genotype-specific mutations of genes were present in the Dutch strains. Furthermore, a relatively larger number of non-synonymous mutations were present in critical genes of the CbNL01 strains compared to the CbNL12 strains.

\section{Changes in LPS encoding genes and its effect on phase variation}

Repeated in vitro passages of $C$. burnetii strains have been shown to induce antigenic variation and loss of virulence characteristics due to the transition into a truncated LPS structure. This truncation of LPS is associated with chromosomal deletions of O-antigen coding genes, located in a $38 \mathrm{~kb}$ region in the genome resulting in antigenic phase variation from phase I to phase II $[27,55,56]$. The genome sequences established in this study, were compared with the LPS encoding region (CBU0676 to CBU0706) of NM-phase I (GenBank: AF387640) to visualize deletions in this LPS encoding region. The human strains Henzerling, Heizberg, Schperling and Scurry contained no deletions in this region (Fig 6a). However, a deletion of 201 bp (NM-phase I LPS encoding region: 14604-14805) was consistently observed in this region in all sequenced $(601,2574,701 \mathrm{CbB} 1,18430)$ and database strains (CbB1, CbB18, EV-Cb_BK10) of the CbNL12 genotype (Fig 6b). This deletion corresponded to a portion of CBU_0686 gene (old gene name: JB153-5), a paralog predicted to encode a 2-Oxoacid dehydrogenase [56]. As a paralogous gene, deletion of a portion of this gene might not affect the LPS 
synthesis as the function might be compensated by the other gene in the genome. Similarly, a deletion of 355 bp (NM-phase I LPS encoding region: 20476-20830) was consistently observed in this region in all sequenced (NL3262, 602, CbCVIC1, NLhu3345937, 42785537) and database strains (NLLimburg, Cb109, EV-Cb_C13) of the CbNL01 genotype. This deletion corresponded to a portion of CBU_0691 gene (old gene name: JB153-10, Fig 6c and 6d) and is predicted to be involved in virenose synthesis which is one of the unusual sugars only present in the C. burnetii LPS-phase I structure [57].

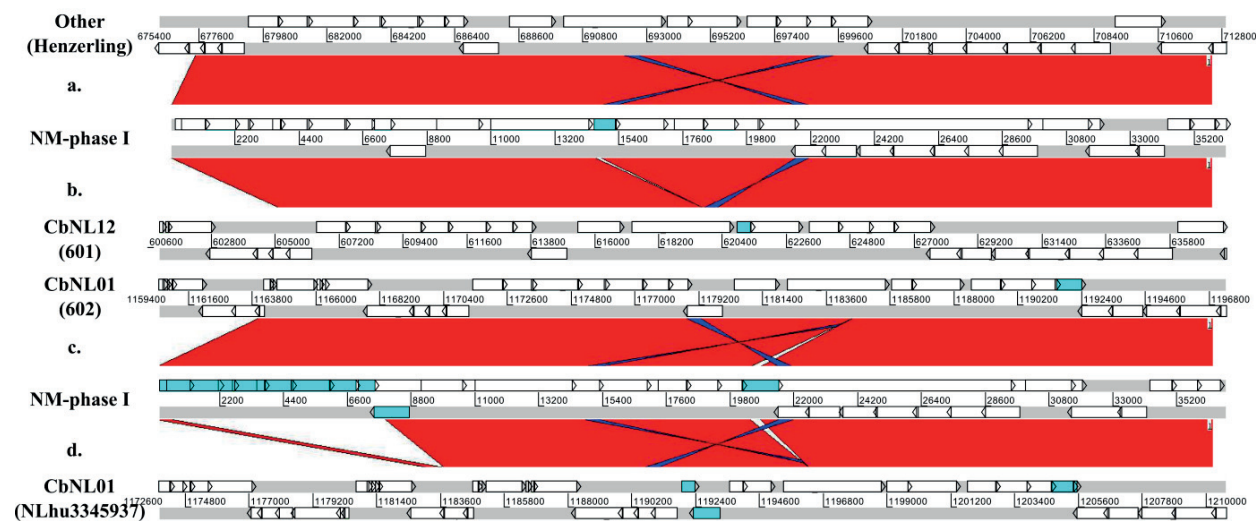

Figure 6. Artemis Comparison Tool representation of the LPS encoding region of 4 C. burnetii strains compared to NM-phase I. Pairwise comparison of sequenced strains with LPS encoding region of NM-phase I. a: comparison of Henzerling and NM-phase I showing no deletions (similarly in other strains: Heizberg, Scurry and Schperling), b: comparison of the CbNL12 strain 601 and NM-phase I showing a deletion (similarly in other CbNL12 strains), c: comparison of the CbNL01 strain 602 and NM-phase I showing a deletion (similarly in other CbNL01 strains) and d: comparison of the CbNL01 strain NLhu3345937 and NM-phase I showing deletions. Red bars indicate same DNA sequence identity, blue bars indicate inverted reads. Deletions of a portion of genes or complete genes in b, c and d are seen as white gaps. Genes involved in a deletion are colored in cyan.

Due to the deletion, CBU_0691 gene is frame-shifted and might consequently not encode for enzymes involved in virenose synthesis. We hypothesize that this deletion of $355 \mathrm{bp}$ may induce the start of phase shifting in CbNL01 strains. Since the genome sequences of 602 (CbNL01 genotype) was obtained from in vitro grown cell cultures, we looked back into our previous functional analysis data of the 602 strain cultured in cells maintaining a low passage number. Our transcriptome measurements showed that the CBU_0691 gene was actively transcribed in cells [27]. Although the deletion of a portion of this gene results in frame shifting of CBU_0691, it is still possible that gene CBU_0691 is transcribed but that no protein product is formed. In addition, a complete gene deletion (corresponding to CBU_0677 to CBU_0682) and a deletion of part of the genes (corresponding to CBU_0676 and CBU_0683) were observed in the NLhu3345937 strain of the CbNL01 genotype. The NLhu3345937 
strain was passaged in cells for only 11 times and already showed deletion of several O-antigen genes, indicating a high rate of phase shifting in this strain under in vitro culture conditions (Fig 8d). Apart from these deletions, non-synonymous mutations in CBU_0688 and CBU_0698 were present in all CbNL01 strains relative to NM. Additionally, mutations in $12 \mathrm{O}$-antigen genes were observed in all CbNL01 and CbNL12 strains, compared to NM (Supplementary Fig 2). Taken together, we observed genotype-specific deletions and mutations in the LPS encoding region at a higher rate in CbNL01 strains than in CbNL12 strains.

\section{Discussion}

In this study we used genome comparisons to detail the extent of genomic diversity of several $C$. burnetii strains. Focus was given to goat and human strains of the Dutch predominant CbNL01 genotype to identify genomic aspects resulting in its anticipated increased virulence characteristics. In all the strains, we firstly performed phylogenome analysis to infer the relationships between the sequenced strains. Further, we analyzed orthologs and mutations among all sequenced strains with respect to the NM reference strain to assess differences between strains at the gene and nucleotide level.

Regardless of whether the sequence data was derived from complete or draft genome sequences, the phylogenetic clustering of the strains was based on the MLVA genotype rather than on host origin. Clustering of the genome sequences was similar to the clustering based on in silico MLVA genotyping. Moreover, a similar genotype-specific clustering of strains was observed based on SNP analysis. The inferred topology of C. burnetii strains is consistent with different analysis methods and also with previous phylogenetic studies based on MLVA and MST genotypic data [6,20,24]. Based on these findings, a close relationship of genotype-specific $C$. burnetii strains was clearly observed. The phylogenetic analysis, as described here, is an improved genotyping tool, besides the MLVA and MST methods, as it uses whole genome sequences for comparisons of strains rather than a few genomic loci. Nevertheless, similar results were obtained with the three techniques. Thus the results of our study validate the traditional genotyping methodologies as sensitive and less-time consuming tools used for outbreak investigations and molecular epidemiology of pathogens.

Among the different Dutch outbreak strains, the NL3262 strain has now a complete genome sequence. The NL-Limburg database strain has a draft genome sequence, although with contigs covering the complete genome of NL3262. Both strains are clonal as the difference between these strains is limited to 8 point mutations. Compared to each other, both strains NL3262 and NL-Limburg have only one DNA rearrangement in their genomes (Fig 5) and contain around 121-130 transposase encoding genes. This is the highest number of transposase genes encoded by any other C. burnetii strain [21]. Upon phylogenetic genome analysis, strains NL3262 and NL-Limburg cluster together in cluster 1a. These strains differ from other CbNL01 strains in cluster $1 \mathrm{~b}$ by point mutations (an average of $12 \mathrm{SNPs}$ ) and annotation of transposons, which are the major contig break regions in these draft 
genome sequences (Fig 1). Thus, based on phylogenetic and SNP analysis, the outbreak goat (NL3262, 602, CbCVIC1) and human strains (NLhu3345937, 42785537, NL-Limburg) of the CbNL01 genotype cluster together and possess almost identical genomes. Furthermore, these data confirm that goat are the source of the Dutch human Q fever outbreak as suggested previously by genotyping (Fig 1 and 2) [6].

Both CbNL01 and CbNL12 strains were isolated in The Netherlands during the outbreak period from 2007- 2010. The CbNL12 genotype strains were the second most prevalent strains after the CbNL01 strains and were only isolated from cattle, goat and sheep, but not from humans. The CbNL12 genome sequence was shown to be similar to the genome sequence of the reference strain NM (Fig 1 and 2). Compared to CbNL01 strains, the CbNL12 strains clustered separately based on phylogenomics (cluster 1 and 2, Fig 1) but the distance between these clusters was very small (MUMi distance 0.022-0.03) indicating closely related genomes. Although the CbNL12 and CbNL01 strains belong to distinct MLVA genotypes, the major differences were based on point mutations (average of 2400 SNPs) and the number of transposase encoding genes. The cattle strains 2574 and $701 \mathrm{CbB} 1$ used in this study are of the CbNL12 genotype and from different geographical locations. The 701CbB1 strain is an archival sample isolated in France and a similar genotype strain (2574) was recently isolated during the Dutch Q fever outbreak period. The cattle strains were clustered together phylogenetically, indicating a close relationship between these strains. In addition, the published genome sequences of 3 strains (Cb_B1, Cb_B18, EV-Cb_BK10) of the CbNL12 genotype, which were isolated from ruminants in France, were also present in the same cluster (Cluster 2, Fig 1). The presence of similar genome strains obtained from various geographical locations may imply a clonal spread of CbNL12 strains over different European countries. This idea is strengthened by the fact that strains of the CbNL12 genotype were identified in consumer cow milk products obtained from various European countries [58]. As the CbNL12 strains are not detected in human samples but are spread over many European countries, it could be hypothesized that humans are less susceptible to CbNL12 genotype strains, decreasing its risk for causing an outbreak in humans.

The core genome of sequenced C. burnetii strains constitutes up to $75 \%$ of the complete gene repertoire of the sequenced $C$. burnetii strains, indicating a conservative nature of evolution. Gene ortholog analysis showed a $98 \%$ overlap between strains of the same genotype which is higher than the overlap between strains of the same host-origin. This indicates that strains of the same genotype are of clonal origin with highly conserved gene content (Fig 4). Unique genes were not identified in the Dutch outbreak strains and other closely related strains (CbNL12, Henzerling, Heizberg), although genotype specific genes were identified (Supplementary Table 2). A search of host-specific genes and signatures (based on point mutation) didn't yield any results, indicating that host-specific genes and mutation were absent. Thus, genotype-specific changes were clearly observed in strains, whereas hostspecific changes in the strains could not be observed. Based on these findings, the observed phenotypic differences in clinical manifestations of Q fever in natural hosts, goats (abortion), cattle 
(rarely clinical symptoms), human acute infections (asymptomatic) and human chronic infections (endocarditis), is most probably due to host-specific (immune) responses to an infection with $C$. burnetii or other pathogen-related factors (such as host-tropism) which are not known yet.

The difference between the genome sequences of the Dutch outbreak strains and the reference strain NM were based on point mutations, small deletions in parts of genes and a few deletions of complete genes in the CbNL01 strains (Supplementary Fig 2 and 7). Novel genes, potentially associated with increased bacterial virulence, were absent in the CbNL01 strains. This suggests that modifications of existing genes have given rise to the high-virulent features of the outbreak strains rather than the acquisition of novel genetic factors by $C$. burnetii. The present findings support previous observations that genomes of $C$. burnetii lack broad genetic variance, due to absence of DNA exchange machinery and the intracellular lifestyle, which limits opportunities for genetic exchange $[33,36,50]$. Upon assessing the variants in the Dutch strains, relative to NM, genotype-specific mutations in the CbNL01 strains were predominantly found in hypothetical membrane proteins, transporter proteins, translation proteins, DNA repair protein encoding genes and T4SS genes (Supplementary Fig 2). Our study showed a large number of mutations in membrane protein encoding genes of CbNL01 strains, relative to the CbNL12 strains. Amino acid changes in these membrane proteins may lead to a distinct antigenic profile of the CbNL01 strains, which may result in a change in immune evasion efficiencies. Based on these findings, we speculate that a shift in the bacterial antigenic repertoire has resulted in the increased zoonotic potential of the outbreak strains. Furthermore, mutations in transporter genes involved majorly in metabolite transportation, could be crucial for efficiently assessing host-derived nutrients to adapt in the host hostile environments. Additionally, a large number of mutations in the protein synthesis machinery could be a novel mechanism for adaptation of CbNL01 strains to the host environment. These mutations may result in altered levels of protein synthesis, thereby influencing the growth rate of the bacterium in the host niche [33]. Increased numbers of mutations in DNA-repair genes of the CbNL01 strains could be related to an altered/increased capability of these strains to fight against oxidative stress. This feature might aid in efficient maintenance of the bacterial cell integrity in harsh environments of host cell PV. Finally, the mutations in genes encoding virulence related effector proteins and the T4SS proteins delivering system could contribute to an increased/altered virulence potential of CbNL01 strains by an enhanced/altered protein export. This could be beneficial for C. burnetii in effective manipulation of host components or host intracellular processes to enhance successful replication and persistent infections in hosts [52].

Our study shows that non-synonymous SNPs in a number of membrane protein encoding genes, transporter genes, genes encoding virulence-related effector proteins and $\mathrm{O}$-antigen genes of CbNL01 strains resulted in gene frame-shifts (Supplementary Fig 2). These frameshifts result in the gain of pseudo-genes, which could be a mechanism of "genome reduction". It could be speculated that lost gene functions might be compensated by other genes. Alternatively, the bacteria might not require 
this function anymore due to its intimate relationship with host cells [59]. The presence of frame-shift mutations might increase the likelihood of the removal of whole genes by DNA deletion processes [60], as evidenced by the observed deletion of an ankyrin repeat protein ortholog to CBU_0072 in all CbNL01 strains (Supplementary Fig 2). It could be speculated that loss of genes can be a crucial mechanism for specialization of the bacteria to survive in a specific niche as seen previously for other bacterial species $[61,62]$. On the whole, our findings favor the reductive evolution of genomes in the CbNL01 strains, where increased gene loss could have led to bacterial specialization in a host niche [62].

In this study we focused on the O-antigen encoding region, the most prominently known virulence-related genomic region of $C$. burnetii, which is prone to deletions resulting in phenotypic phase shifting [56]. The sequenced strains were cultivated both in cell-based and in cell-free systems and the passage numbers were kept at the minimum to avoid phenotypic phase shifting. Although we maintained low passage numbers (passages between 4 to 14), the CbNL01 and CbNL12 genotype strains showed partial gene deletions in this region. Such deletions were not observed in the remaining human strains used in this study (Schperling, Scurry, Henzerling and Heizberg) (Fig 6a). Deletion of portion of the CBU_0691 and CBU_0686 genes in CbNL01 and CbNL12 strains, respectively were identified. We anticipate that these deletions could have a significant effect on the structure and function of LPS as these genes are primarily involved in LPS biosynthesis. Although, these genes are polymorphic and frameshifted, the Dutch strains (CbNL01: NL3262, 602 and CbNL12: 601) were shown to be virulent in a mice virulence bioassay [27]. Hence, based on these observations the effect of genotype-specific deletions in the LPS encoding region and its effect on virulence of the Dutch strains are not clearly known and should be the further investigated.

Transposable elements are present in C. burnetii genomes and contribute to genome plasticity [52]. The Dutch outbreak strains (NL3262, NL-Limburg) encode more than 100 transposase genes which is the highest number of transposase genes found in C. burnetii (30 in NM, Z3055, Dugway; 59 in Cbuk_Q154 and 40 in CbuG_Q212). Although we cannot infer the exact number of transposons in draft genome sequences, we anticipate that the higher number of contigs in the sequenced CbNL01 strains (average of 130) corresponds to the higher number of transposons, as contig ends often map to transposase genes relative to NM genome. Transposases are basically mobile genetic units that exhibit broad diversity in their structure and transposition mechanisms. Movement and accumulation of these elements appear to be a major force shaping C. burnetii genetic diversity [21,36]. The CbNL01 and $\mathrm{NM}$ genomes are similar in the majority of their gene content, but differ significantly in genome structure due to a large number of genome rearrangements. These rearrangements are generated by homologous recombination between IS110 family transposon genes as evidenced by the presence of transposase genes at almost all genome breakpoints (95\%) of NL3262 relative to the NM and Z3055 genomes (Fig 5, Supplementary Table 3). Due to higher number of transposons, the genomes of CbNL01 seem to be highly plastic compared to other strains. Such plasticity of the outbreak strain 
genomes induced by movement of mobile elements could be a potential mechanism for the generation of new adaptive mechanisms to efficiently acclimatize in its intracellular niche $[64,65]$.

In conclusion, the present study provides the first complete study on phylogenetic analysis of Dutch outbreak strains, a few additionally selected strains, and all publicly available C. burnetii genome sequences. Hierarchical clustering based on genome content, the in silico MLVA genotypes and SNP analysis, showed a genotype-specific clustering of strains, showing highly similar genomes of $C$. burnetii within each genotype. The differences between the Dutch outbreak strains relative to the reference NM and CbNL12 strains were mainly based on single nucleotide polymorphisms. We hypothesize that high numbers of transposons, genotype specific polymorphisms in membrane protein encoding genes and virulence related genes could have resulted in a highly flexible genome, an altered antigenic profile and specialized virulence mechanisms. The observed subtle genetic differences form the basis for the increased epidemic potential and the Q fever outbreak in The Netherlands. Finally, the comparative analysis of several $C$. burnetii strains from this study has the potential to provide information on cross-protective vaccine candidates. Additionally, the consistent genotype-specific genetic differences; such as SNPs and deletions could be used as additional markers for better characterization of $C$. burnetii isolate.

\section{Acknowledgements}

We thank Frank Harders, Albert de Boer and Annemieke Dinkla for technical assistance. We also thank Dimitrios Frangoulidis from Bundeswehr institute of Microbiology, Munich, Germany and A. Rodolakis from INRA, France for kindly providing us with Henzerling, Heizberg, Scurry, Schperling and $701 \mathrm{CbB} 1$ C. burnetii strains. 


\section{References}

1. Maurin M, Raoult D. Q fever. Clin. Microbiol. Rev. 1999;12:518-53.

2. Akporiaye ET, Rowatt JD, Aragon AA, Baca OG. Lysosomal response of a murine macrophagelike cell line persistently infected with Coxiella burnetii. Infect. Immun. 1983;40:1155-62.

3. Woldehiwet Z. Q fever (coxiellosis): epidemiology and pathogenesis. Res. Vet. Sci. 2004;77:93100 .

4. Norlander L. Q fever epidemiology and pathogenesis. Microbes Infect. Inst. Pasteur. 2000;2:41724.

5. Palmer NC, Kierstead M, Key DW, Williams JC, Peacock MG, Vellend H. Placentitis and Abortion in Goats and Sheep in Ontario Caused by Coxiella burnetii. Can. Vet. J. 1983;24:60-1.

6. Roest HIJ, Ruuls RC, Tilburg JJHC, Nabuurs-Franssen MH, Klaassen CHW, Vellema P, et al. Molecular epidemiology of Coxiella burnetii from ruminants in Q fever outbreak, the Netherlands. Emerg. Infect. Dis. 2011;17:668-75.

7. To H, Htwe KK, Kako N, Kim HJ, Yamaguchi T, Fukushi H, et al. Prevalence of Coxiella burnetii infection in dairy cattle with reproductive disorders. J. Vet. Med. Sci. 1998;60:859-61.

8. Arricau-Bouvery N, Rodolakis A. Is Q fever an emerging or re-emerging zoonosis? Vet. Res. 2005;36:327-49.

9. Arricau Bouvery N, Souriau A, Lechopier P, Rodolakis A. Experimental Coxiella burnetii infection in pregnant goats: excretion routes. Vet. Res. 2003;34:423-33.

10. Roest H-J, van Gelderen B, Dinkla A, Frangoulidis D, van Zijderveld F, Rebel J, et al. Q Fever in Pregnant Goats: Pathogenesis and Excretion of Coxiella burnetii. PLoS ONE. 2012;7:e48949.

11. Raoult D, Marrie T, Mege J. Natural history and pathophysiology of Q fever. Lancet Infect. Dis. 2005;5:219-26.

12. Mazokopakis EE, Karefilakis CM, Starakis IK. Q fever endocarditis. Infect. Disord. Drug Targets. 2010;10:27-31.

13. Roest HIJ, Tilburg JJHC, van der Hoek W, Vellema P, van Zijderveld FG, Klaassen CHW, et al. The Q fever epidemic in The Netherlands: history, onset, response and reflection. Epidemiol. Infect. 2011;139:1-12.

14. Hammerl JA, Mertens K, Sprague LD, Hackert VH, Buijs J, Hoebe CJ, et al. First Draft Genome Sequence of a Human Coxiella burnetii Isolate, Originating from the Largest Q Fever Outbreak Ever Reported, the Netherlands, 2007 to 2010. Genome Announc. [Internet]. 2015 [cited 2016 Jan 29];3. Available from: http://www.ncbi.nlm.nih.gov/pmc/articles/PMC4424315/

15. Kuley R, Smith HE, Janse I, Harders FL, Baas F, Schijlen E, et al. First Complete Genome Sequence of the Dutch Veterinary Coxiella burnetii Strain NL3262, Originating from the Largest Global Q Fever Outbreak, and Draft Genome Sequence of Its Epidemiologically Linked Chronic Human Isolate NLhu3345937. Genome Announc. [Internet]. 2016 [cited 2016 Jun 28];4. Available from: http://www.ncbi.nlm.nih.gov/pmc/articles/PMC4841129/

16. Enserink M. Questions Abound in Q-Fever Explosion in the Netherlands. Science. 2010;327:2667. 
17. Kampschreur LM, Hagenaars JCJP, Wielders CCH, Elsman P, Lestrade PJ, Koning OHJ, et al. Screening for Coxiella burnetii seroprevalence in chronic Q fever high-risk groups reveals the magnitude of the Dutch Q fever outbreak. Epidemiol. Infect. 2013;141:847-51.

18. van der Hoek W, Dijkstra F, Schimmer B, Schneeberger PM, Vellema P, Wijkmans C, et al. Q fever in the Netherlands: an update on the epidemiology and control measures. Euro Surveill. 2010;15.

19. Mori M, Boarbi S, Michel P, Bakinahe R, Rits K, Wattiau P, et al. In Vitro and In Vivo Infectious Potential of Coxiella burnetii: A Study on Belgian Livestock Isolates. PLoS ONE. 2013;8:e67622.

20. Tilburg JJHC, Rossen JWA, van Hannen EJ, Melchers WJG, Hermans MHA, van de Bovenkamp $\mathrm{J}$, et al. Genotypic diversity of Coxiella burnetii in the 2007-2010 Q fever outbreak episodes in The Netherlands. J. Clin. Microbiol. 2012;50:1076-8.

21. Beare PA, Unsworth N, Andoh M, Voth DE, Omsland A, Gilk SD, et al. Comparative Genomics Reveal Extensive Transposon-Mediated Genomic Plasticity and Diversity among Potential Effector Proteins within the Genus Coxiella. Infect. Immun. 2009;77:642-56.

22. Gilk SD. Role of Lipids in Coxiella burnetii Infection. In: Toman R, Heinzen RA, Samuel JE, Mege J-L, editors. Coxiella Burn. Recent Adv. New Perspect. Res. Q Fever Bact. [Internet]. Springer Netherlands; 2012 [cited 2014 Oct 25]. p. 199-213. Available from: http://link.springer.com/chapter/10.1007/978-94-007-4315-1_10

23. Kuley R, Bossers-deVries R, Smith HE, Smits MA, Roest HIJ, Bossers A. Major differential gene regulation in Coxiella burnetii between in vivo and in vitro cultivation models. BMC Genomics. 2015; 16:953.

24. Tilburg JJHC, Roest H-JIJ, Buffet S, Nabuurs-Franssen MH, Horrevorts AM, Raoult D, et al. Epidemic Genotype of Coxiella burnetii among Goats, Sheep, and Humans in the Netherlands. Emerg. Infect. Dis. 2012;18:887-9.

25. Omsland A, Beare PA, Hill J, Cockrell DC, Howe D, Hansen B, et al. Isolation from Animal Tissue and Genetic Transformation of Coxiella burnetii Are Facilitated by an Improved Axenic Growth Medium ${ }^{\nabla}$. Appl. Environ. Microbiol. 2011;77:3720-5.

26. Tang F, Bossers A, Harders F, Lu C, Smith H. Comparative genomic analysis of twelve Streptococcus suis (pro)phages. Genomics. 2013;101:336-44.

27. Kuley R, Smith HE, Frangoulidis D, Smits MA, Jan Roest HI, Bossers A. Cell-Free Propagation of Coxiella burnetii Does Not Affect Its Relative Virulence. PLoS ONE [Internet]. 2015 [cited 2015 Apr 12];10. Available from: http://www.ncbi.nlm.nih.gov/pmc/articles/PMC4368683/

28. Glazunova O, Roux V, Freylikman O, Sekeyova Z, Fournous G, Tyczka J, et al. Coxiella burnetii Genotyping. Emerg. Infect. Dis. 2005;11:1211-7.

29. Bankevich A, Nurk S, Antipov D, Gurevich AA, Dvorkin M, Kulikov AS, et al. SPAdes: A New Genome Assembly Algorithm and Its Applications to Single-Cell Sequencing. J. Comput. Biol. 2012;19:455-77.

30. Kent WJ. BLAT--the BLAST-like alignment tool. Genome Res. 2002;12:656-64.

31. Langmead B, Salzberg SL. Fast gapped-read alignment with Bowtie 2. Nat. Methods. 2012;9:3579 . 
32. D'Amato F, Eldin C, Georgiades K, Edouard S, Delerce J, Labas N, et al. Loss of TSS1 in hypervirulent Coxiella burnetii 175, the causative agent of Q fever in French Guiana. Comp. Immunol. Microbiol. Infect. Dis. 2015;41:35-41.

33. D'Amato F, Rouli L, Edouard S, Tyczka J, Million M, Robert C, et al. The genome of Coxiella burnetii Z3055, a clone linked to the Netherlands Q fever outbreaks, provides evidence for the role of drift in the emergence of epidemic clones. Comp. Immunol. Microbiol. Infect. Dis. 2014;37:281-8.

34. Rouli L, Rolain J-M, El Filali A, Robert C, Raoult D. Genome sequence of Coxiella burnetii 109, a doxycycline-resistant clinical isolate. J. Bacteriol. 2012;194:6939.

35. Sidi-Boumedine K, Ellis RJ, Adam G, Prigent M, Angen $\varnothing$, Aspán A, et al. Draft Genome Sequences of Six Ruminant Coxiella burnetii Isolates of European Origin. Genome Announc. 2014;2:e00285-14.

36. Beare PA, Samuel JE, Howe D, Virtaneva K, Porcella SF, Heinzen RA. Genetic Diversity of the Q Fever Agent, Coxiella burnetii, Assessed by Microarray-Based Whole-Genome Comparisons. J. Bacteriol. 2006;188:2309-24.

37. Walter MC, Öhrman C, Myrtennäs K, Sjödin A, Byström M, Larsson P, et al. Genome sequence of Coxiella burnetii strain Namibia. Stand. Genomic Sci. 2014;9:22.

38. Walter MC, Vincent GA, Stenos J, Graves S, Frangoulidis D. Genome Sequence of Coxiella burnetii Strain AuQ01 (Arandale) from an Australian Patient with Acute Q Fever. Genome Announc. $2014 ; 2$.

39. Arricau-Bouvery N, Hauck Y, Bejaoui A, Frangoulidis D, Bodier CC, Souriau A, et al. Molecular characterization of Coxiella burnetii isolates by infrequent restriction site-PCR and MLVA typing. BMC Microbiol. 2006;6:38.

40. Deloger M, El Karoui M, Petit M-A. A Genomic Distance Based on MUM Indicates Discontinuity between Most Bacterial Species and Genera. J. Bacteriol. 2009;191:91-9.

41. Gascuel O. BIONJ: an improved version of the NJ algorithm based on a simple model of sequence data. Mol. Biol. Evol. 1997;14:685-95.

42. Kurtz S, Phillippy A, Delcher AL, Smoot M, Shumway M, Antonescu C, et al. Versatile and open software for comparing large genomes. Genome Biol. 2004;5:R12.

43. Huson DH, Bryant D. Application of Phylogenetic Networks in Evolutionary Studies. Mol. Biol. Evol. 2006;23:254-67.

44. Li H, Handsaker B, Wysoker A, Fennell T, Ruan J, Homer N, et al. The Sequence Alignment/Map format and SAMtools. Bioinforma. Oxf. Engl. 2009;25:2078-9.

45. Sievers F, Wilm A, Dineen D, Gibson TJ, Karplus K, Li W, et al. Fast, scalable generation of highquality protein multiple sequence alignments using Clustal Omega. Mol. Syst. Biol. 2011;7:539.

46. Darling ACE, Mau B, Blattner FR, Perna NT. Mauve: multiple alignment of conserved genomic sequence with rearrangements. Genome Res. 2004;14:1394-403.

47. Lechner M, Findeiss S, Steiner L, Marz M, Stadler PF, Prohaska SJ. Proteinortho: detection of (co)orthologs in large-scale analysis. BMC Bioinformatics. 2011;12:124.

48. Carver TJ, Rutherford KM, Berriman M, Rajandream M-A, Barrell BG, Parkhill J. ACT: the Artemis comparison tool. Bioinformatics. 2005;21:3422-3. 
49. Thompson JD, Higgins DG, Gibson TJ. CLUSTAL W: improving the sensitivity of progressive multiple sequence alignment through sequence weighting, position-specific gap penalties and weight matrix choice. Nucleic Acids Res. 1994;22:4673-80.

50. Seshadri R, Paulsen IT, Eisen JA, Read TD, Nelson KE, Nelson WC, et al. Complete genome sequence of the Q-fever pathogen Coxiella burnetii. Proc. Natl. Acad. Sci. U. S. A. 2003;100:545560 .

51. Willems H, Ritter M, Jäger C, Thiele D. Plasmid-homologous sequences in the chromosome of plasmidless Coxiella burnetii Scurry Q217. J. Bacteriol. 1997;179:3293-7.

52. Beare PA, Unsworth N, Andoh M, Voth DE, Omsland A, Gilk SD, et al. Comparative genomics reveal extensive transposon-mediated genomic plasticity and diversity among potential effector proteins within the genus Coxiella. Infect. Immun. 2009;77:642-56.

53. Mertens K, Samuel JE. Defense mechanisms against oxidative stress in Coxiella burnetii: adaptation to a unique intracellular niche. Adv. Exp. Med. Biol. 2012;984:39-63.

54. van Schaik EJ, Chen C, Mertens K, Weber MM, Samuel JE. Molecular pathogenesis of the obligate intracellular bacterium Coxiella burnetii. Nat. Rev. Microbiol. 2013;11:561-73.

55. Denison AM, Massung RF, Thompson HA. Analysis of the O-antigen biosynthesis regions of phase II isolates of Coxiella burnetii. FEMS Microbiol. Lett. 2007;267:102-7.

56. Hoover TA, Culp DW, Vodkin MH, Williams JC, Thompson HA. Chromosomal DNA deletions explain phenotypic characteristics of two antigenic variants, phase II and RSA 514 (crazy), of the Coxiella burnetii nine mile strain. Infect. Immun. 2002;70:6726-33.

57. Toman R, Škultéty L, Ftáček P, Hricovíni M. NMR study of virenose and dihydrohydroxystreptose isolated from Coxiella burnetii phase I lipopolysaccharide. Carbohydr. Res. 1998;306:291-6.

58. Tilburg JJHC, Roest HJIJ, Nabuurs-Franssen MH, Horrevorts AM, Klaassen CHW. Genotyping reveals the presence of a predominant genotype of Coxiella burnetii in consumer milk products. J. Clin. Microbiol. 2012;50:2156-8.

59. Merhej V, Royer-Carenzi M, Pontarotti P, Raoult D. Massive comparative genomic analysis reveals convergent evolution of specialized bacteria. Biol. Direct. 2009;4:13.

60. Kuo C-H, Ochman H. The Extinction Dynamics of Bacterial Pseudogenes. PLOS Genet. 2010;6:e1001050.

61. Rolain JM, Vayssier-Taussat M, Saisongkorh W, Merhej V, Gimenez G, Robert C, et al. Partial Disruption of Translational and Posttranslational Machinery Reshapes Growth Rates of Bartonella birtlesii. mBio [Internet]. 2013 [cited 2016 Nov 28];4. Available from: http://www.ncbi.nlm.nih.gov/pmc/articles/PMC3638310/

62. Georgiades K, Raoult D. Genomes of the Most Dangerous Epidemic Bacteria Have a Virulence Repertoire Characterized by Fewer Genes but More Toxin-Antitoxin Modules. PLOS ONE. 2011;6:e17962.

63. Thompson HA, Hoover TA, Vodkin MH, Shaw EI. Do chromosomal deletions in the lipopolysaccharide biosynthetic regions explain all cases of phase variation in Coxiella burnetii strains? An update. Ann. N. Y. Acad. Sci. 2003;990:664-70. 
64. Hall BG. Is the occurrence of some spontaneous mutations directed by environmental challenges? New Biol. 1991;3:729-33.

65. Blot M. Transposable elements and adaptation of host bacteria. Genetica. 1994;93:5-12. 


\section{Supplementary files}

a.

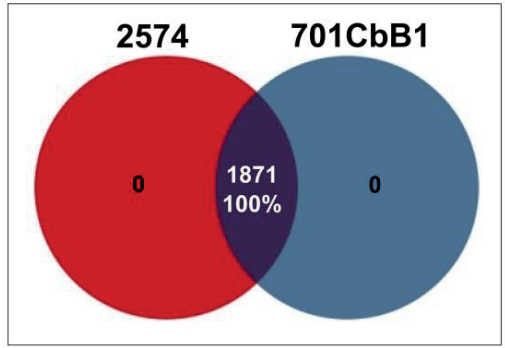

b.

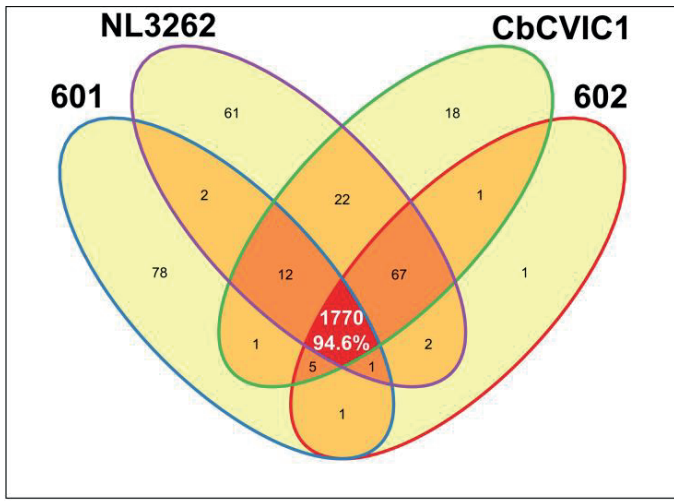

c.

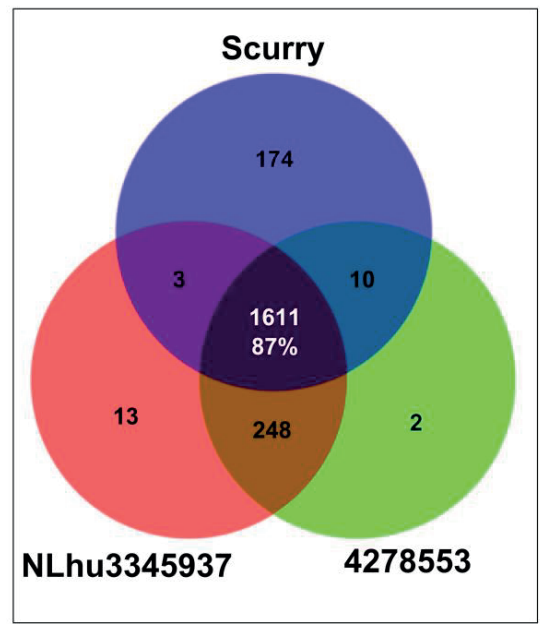

d.

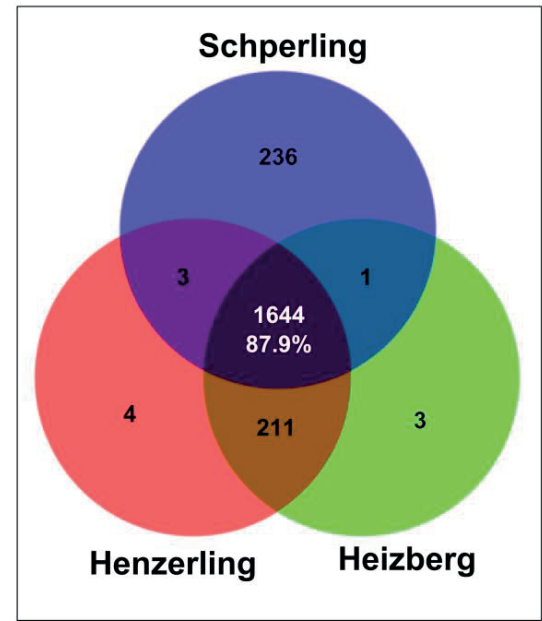

Figure S1. Edwards-Venn diagrams showing the number of orthologs and unique predicted coding proteins among strains originated from same host species.

Number of ortholog genes shared between a) cattle-derived strains b) goat-derived strains c) human (acute infected patients)-derived strains and d) human (chronic infected patients)-derived strains is represented by Edwards-Venn diagrams. Transposase coding proteins were included in the analysis and pseudogenes were scored as absent.

Figure S2. Heat map showing Genes affected (deleted and mutated) in CbNL01 and CbNL12 strains with respect to the reference strain NM.

Deleted genes

Mutated genes (Genes with non-synonomous SNP) with respect to Reference NM genome

Non-Mutated genes with respect to Reference NM genome

frameshifted mutated genes 


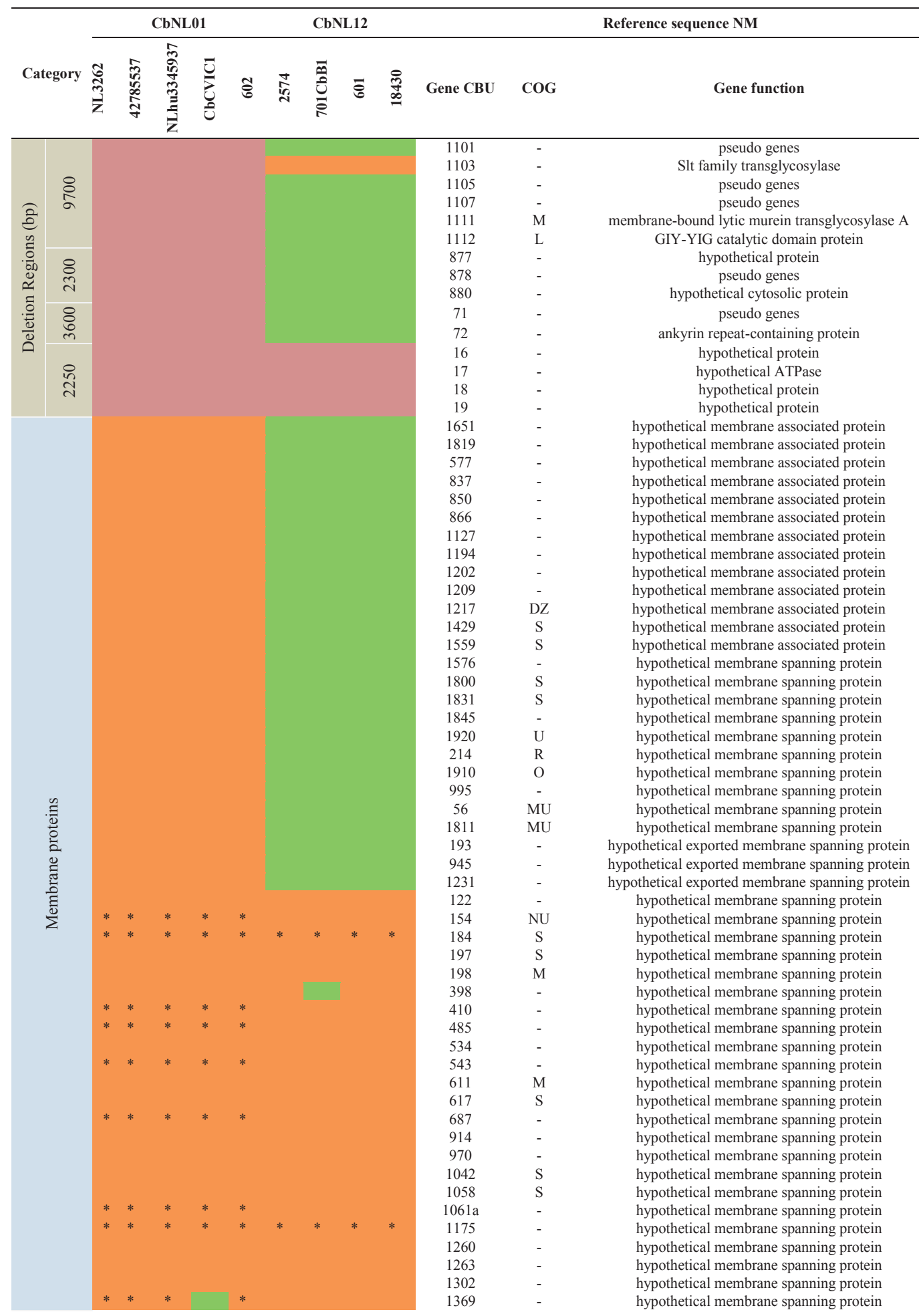




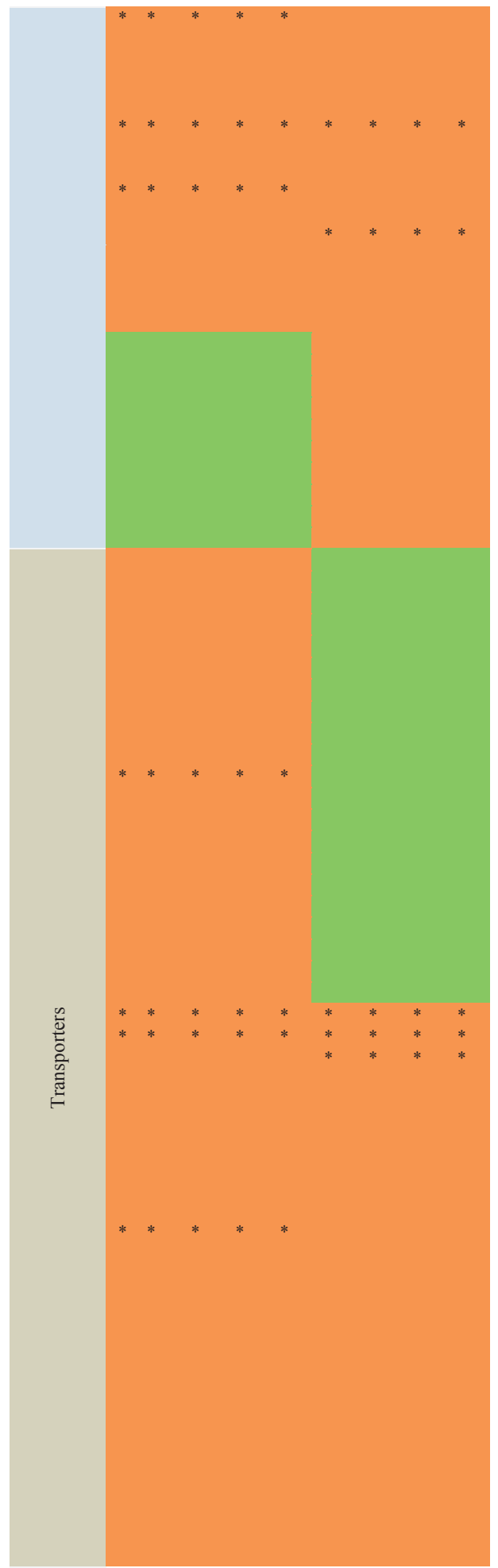

hypothetical outer membrane protein inner membrane protein oxaA integral membrane protein

membrane endopeptidase, M50 family OmpA-like transmembrane domain protein outer membrane lipoprotein outer membrane lipoprotein LolB outer membrane protein outer membrane protein

outer membrane protein assembly complex outer membrane protein, OMP85 family protein putative cytochrome c oxidase, membrane subunit TolC family type I secretion outer membrane protein type 4 pili biogenesis protein

type I secretion outer membrane protein

hypothetical membrane associated protein hypothetical membrane spanning protein hypothetical membrane spanning protein hypothetical membrane spanning protein hypothetical exported membrane spanning protein MarC membrane protein

OmpA-like transmembrane domain protein

OmpA-like transmembrane domain protein outer membrane porin $\mathrm{P} 1$

outer-membrane lipoproteins carrier protein

$\mathrm{ABC}$ transporter ATP-binding protein

$\mathrm{ABC}$ transporter ATP-binding protein

$\mathrm{ABC}$ transporter ATP-binding protein $\mathrm{ABC}$ transporter

$\mathrm{ABC}$ transporter permease protein arginine $\mathrm{ABC}$ transporter

$\mathrm{Bcr} / \mathrm{CflA}$ subfamily drug resistance transporter D-methionine $\mathrm{ABC}$ transporter, ATP-binding protein D-methionine $\mathrm{ABC}$ transporter major facilitator transporter

oligopeptide $\mathrm{ABC}$ transporter, permease protein phosphate transporter proline/betaine transporter

transporter, divalent anion:sodium symporter transporter, MFS superfamily transporter, MFS superfamily transporter, MFS superfamily transporter, MFS superfamily transporter, MFS superfamily peptide $\mathrm{ABC}$ transporter, permease protein zinc uptake transporter

multidrug resistance transporter, Bcr family EmrB/QacA family drug resistance transporter

low-affinity inorganic phosphate transporter

transporter, monovalent cation:proton antiporter-2 di-/tripeptide transporter

AcrB/AcrD/AcrF family transporter

RND family efflux transporter MFP subunit $\mathrm{ABC}$ transporter permease

$\mathrm{EmrB} / \mathrm{QacA}$ family drug resistance transporter RND family efflux transporter MFP subunit

AcrB/AcrD/AcrF family transporter

$\mathrm{ABC}$ transporter permease/ATP-binding protein lipid $\mathrm{ABC}$ transporter permease/ATP-binding protein transporter, MFS superfamily transporter

OPT family oligopeptide transporter cation diffusion facilitator family transporter export $\mathrm{ABC}$ transporter permease protein $\mathrm{ABC}$ transporter

transporter, major facilitator family transporter, MFS superfamily amino acid permease

amino acid $\mathrm{ABC}$ transporter, permease protein amino acid permease amino acid antiporter

tryptophan/tyrosine permease family protein 


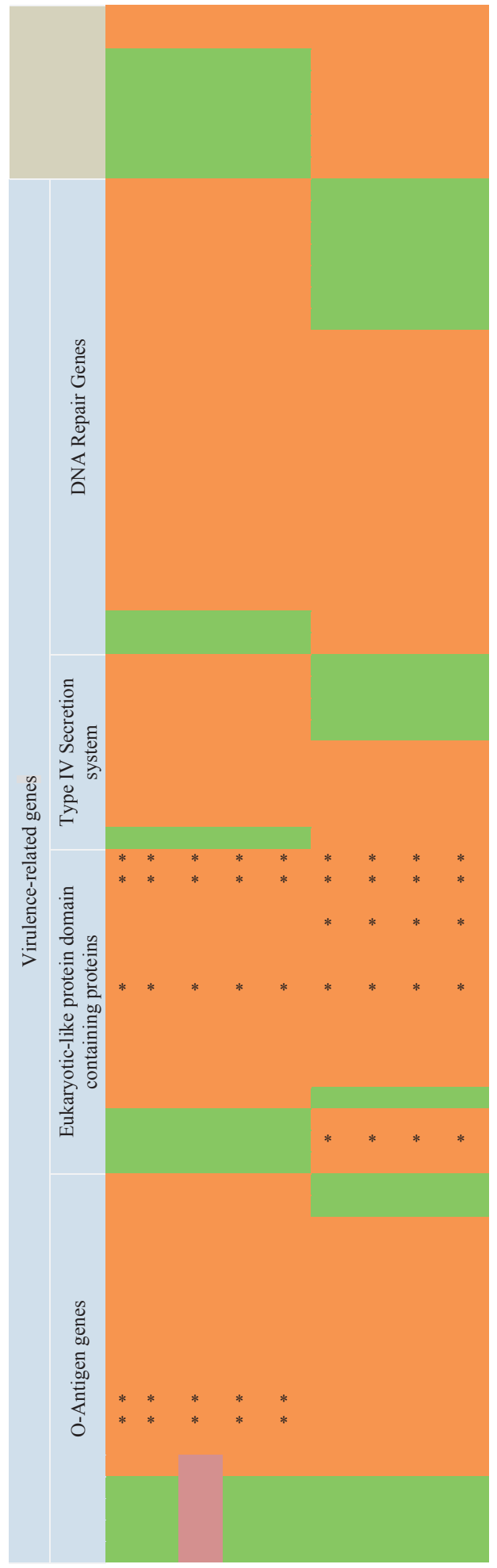

\begin{tabular}{|c|c|}
\hline 1796 & E \\
\hline 1798 & E \\
\hline 1093 & V \\
\hline 481 & E \\
\hline 1179 & G \\
\hline 265 & G \\
\hline 649 & R \\
\hline 484 & E \\
\hline 305 & LK \\
\hline 1083 & L \\
\hline 1229 & $\mathrm{~S}$ \\
\hline 1243 & L \\
\hline 1422 & $\mathrm{O}$ \\
\hline 1148 & LK \\
\hline 557 & L \\
\hline 271 & L \\
\hline 274 & L \\
\hline 506 & L \\
\hline 542 & $\mathrm{~L}$ \\
\hline 1056 & L \\
\hline 1297 & L \\
\hline 2054 & $\mathrm{~L}$ \\
\hline 297 & $\mathrm{~L}$ \\
\hline 1795 & L \\
\hline 518 & $\mathrm{~L}$ \\
\hline 1185 & L \\
\hline 500 & $\mathrm{~L}$ \\
\hline 1337 & L \\
\hline 1476 & K \\
\hline 1697 & L \\
\hline 1651 & - \\
\hline 1650 & - \\
\hline 1645 & $\mathrm{NU}$ \\
\hline 1642 & - \\
\hline 1631 & M \\
\hline 1628 & $\mathrm{~S}$ \\
\hline 1627 & $\mathrm{~S}$ \\
\hline 1648 & $\mathrm{~S}$ \\
\hline 1643 & $\mathrm{~S}$ \\
\hline A0016 & - \\
\hline A0006 & - \\
\hline 1206 & - \\
\hline 599 & $P$ \\
\hline 701 & $P$ \\
\hline 1292 & $\mathrm{R}$ \\
\hline $1253 \mathrm{~b}$ & - \\
\hline 175 & $\mathrm{~T}$ \\
\hline 1136 & $\mathrm{R}$ \\
\hline 1457 & $\mathrm{R}$ \\
\hline 295 & - \\
\hline 1217 & DZ \\
\hline A0013 & - \\
\hline 1158 & - \\
\hline 547 & $\mathrm{R}$ \\
\hline 698 & - \\
\hline 688 & MG \\
\hline 701 & $\mathrm{P}$ \\
\hline 700 & $P$ \\
\hline 695 & - \\
\hline 694 & - \\
\hline 693 & C \\
\hline 689 & M \\
\hline 685 & - \\
\hline 706 & - \\
\hline 687 & - \\
\hline 691 & - \\
\hline 701 & $P$ \\
\hline 678 & $\mathrm{M}$ \\
\hline 676 & MG \\
\hline 683 & 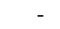 \\
\hline 682 & - \\
\hline 681 & MG \\
\hline
\end{tabular}

amino acid permease family protein amino acid permease family protein

AcrB/AcrD/AcrF family transporter arginine $\mathrm{ABC}$ transporter $\mathrm{ATP}$-binding protein $\mathrm{Bcr} / \mathrm{CflA}$ subfamily drug resistance transporter glucose/galactose transporter riboflavin transporter

amino acid $\mathrm{ABC}$ transporter, permease protein RecG-like helicase

DNA mismatch repair enzyme (predicted ATPase) hypothetical protein

Exonuclease VII, large subunit

Predicted ATP-dependent serine protease

Transcription-repair coupling factor

DNA polymerase III, delta subunit

Single-stranded DNA-binding protein

Excinuclease ATPase subunit

Single-stranded DNA-specific exonuclease NAD-dependent DNA ligase

Mismatch repair ATPase (MutS family)

ATPase involved in DNA repair

Superfamily I DNA and RNA helicases

Exonuclease III

DNA polymerase I

Helicase subunit of the DNA excision repair complex Nuclease subunit of the excinuclease complex

ATPase involved in DNA replication

DNA polymerase III, alpha subunit Transcriptional regulator

Predicted EndoIII-related endonuclease hypothetical membrane associated protein IcmW

Dot/Icm secretion system ATPase DotB IcmS

ompA family protein

IcmK

IcmE

DotA protein

$$
\text { DotD }
$$

CbhE protein

hypothetical protein

delta(24(24(1)))-sterol reductase 3'(2'),5'-bisphosphate nucleotidase

$3^{\prime}\left(2^{\prime}\right), 5^{\prime}$-bisphosphate nucleotidase ankyrin repeat-containing protein ankyrin repeat-containing protein serine/threonine kinase protein

enhanced entry protein enhC

tetratricopeptide repeat family protein hypothetical protein

hypothetical membrane spanning protein hypothetical protein sterol delta-7-reductase

TPR domain-containing protein hypothetical protein

GDP-L-fucose synthase

$3^{\prime}\left(2^{\prime}\right), 5^{\prime}$-bisphosphate nucleotidase

bifunctional sulfate adenylyltransferase subunit 1 hypothetical protein glycosyltransferase

putative pyruvate dehydrogenase

GDP-mannose 4,6 dehydratase hypothetical protein hypothetical protein

hypothetical exported membrane spanning protein methyltransferase

3'(2'),5'-bisphosphate nucleotidase

ADP-heptose synthase, putative

NAD dependent epimerase/dehydratase Pseudo gene

hypothetical protein hypothetical protein 


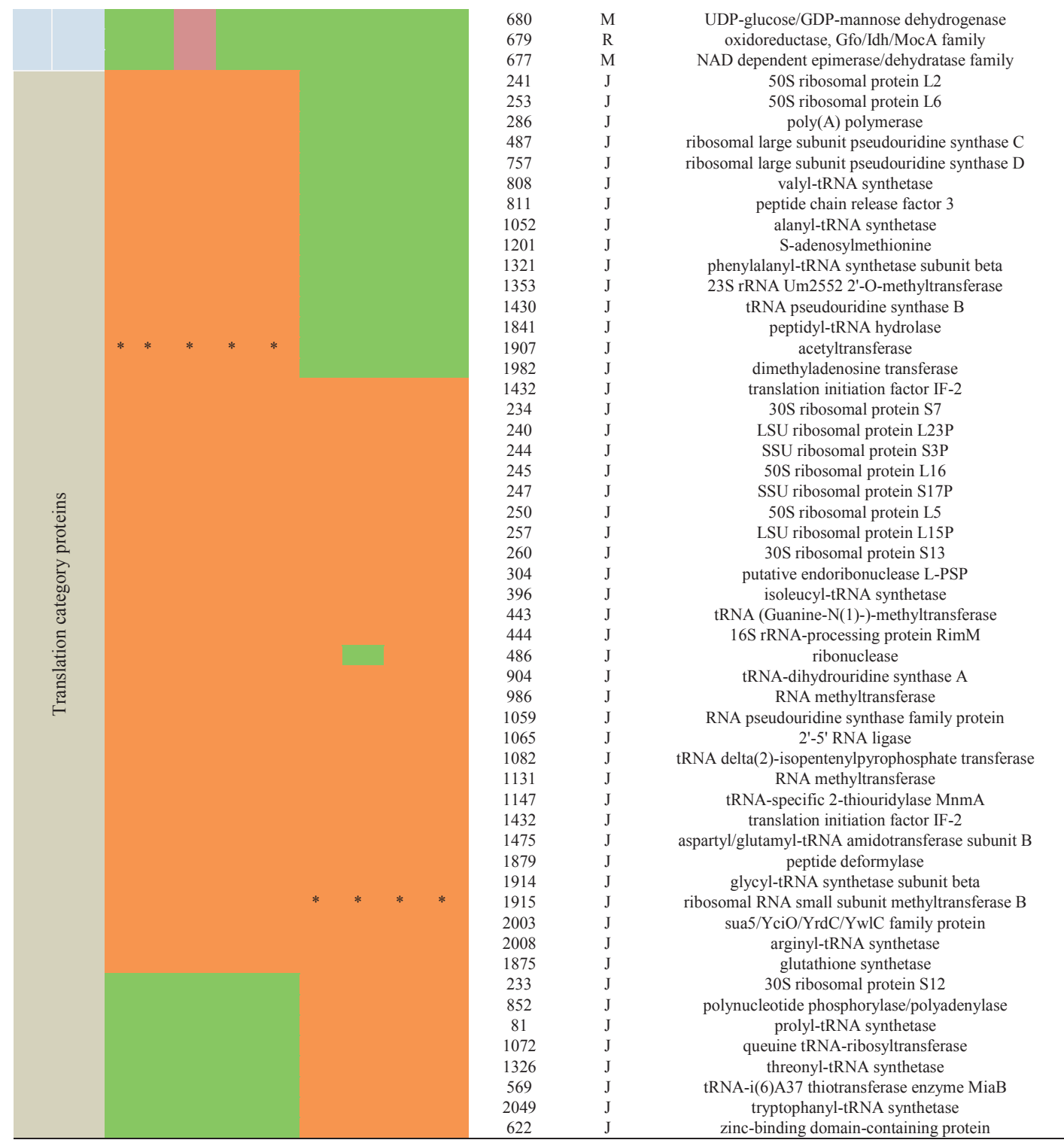


Table S1. List of orthologs in sequenced C. burnetii along with proteins that are ortholog with reference NM as assessed by proteinortho software.

The non-orthologs with reference NM are represented as "-". These genes include point mutations and small insertion/ deletion of $<50$ bp in existing pseudo genes of NM (pseudo genes are not included in the otholog analysis). Annotation of pseudo genes of NM and COG categories of orthologs are mentioned in the table.

Table S1 available on request from alex.bossers@wur.n1

Table S2. List of genotype-specific orthologs in sequenced C. burnetii strains as assessed by proteinortho software.

Non-orthologs between strains of different genotype as well as non orthologs with reference NM strain are represented as "-". These genotype-specific genes include point mutations and small insertion/ deletion of $<50 \mathrm{bp}$ in existing pseudo genes of NM (pseudo genes are not included in the otholog analysis) or contain partial ORF relative to complete ORF in NM. Pseudo genes and complete ORF of NM are mentioned in the table.

Table S2 available on request from alex.bossers@wur.n1 
Table S3. NM and Z3055 genome syntenic blocks relative to NL3262 and flanking transposases in NL3262 strain

\begin{tabular}{|c|c|c|c|c|c|}
\hline Strain & $\begin{array}{l}\text { Syntenic } \\
\text { block } \\
\text { No. }\end{array}$ & $\begin{array}{l}\text { Syntenic block other strain } \\
\text { gene aliases }^{d}\end{array}$ & $\begin{array}{l}\text { Syntenic block } \\
\text { NL3262 gene } \\
\text { aliases }^{d}\end{array}$ & $\begin{array}{l}\text { NL3262 } \\
5^{\prime} \text { transposase } \\
\text { ORF alias }\end{array}$ & $\begin{array}{l}\text { NL3262 } \\
\text { 3' transposase } \\
\text { ORF alias }\end{array}$ \\
\hline \multirow[t]{19}{*}{$\mathbf{N M}$} & 1 & CBU_0282a to CBU_0041 & $\begin{array}{l}\text { AUR58_00225 to } \\
\text { AUR58_01480 }\end{array}$ & AUR58_11620 ${ }^{b}$ & AUR58_01490 \\
\hline & 2 & CBU_1960 to CBU_2095 & $\begin{array}{l}\text { AUR58_01495 to } \\
\text { AUR58_02180 }\end{array}$ & AUR58_01490 & AUR58_02185 \\
\hline & 3 & CBU_2095a to CBU_0039 & $\begin{array}{l}\text { AUR58_02195 to } \\
\text { AUR58_02400 }\end{array}$ & AUR58_02185 & AUR58_02405 ${ }^{c}$ \\
\hline & 4 & CBU_1960 to CBU_1657 & $\begin{array}{l}\text { AUR58_02415 to } \\
\text { AUR58_04055 }\end{array}$ & AUR58_02405 ${ }^{c}$ & AUR58_04065 \\
\hline & 5 & CBU_1641 to CBU_1655 & $\begin{array}{l}\text { AUR58_04075 to } \\
\text { AUR58_04160 }\end{array}$ & AUR58_04065 & AUR58_04165 \\
\hline & 6 & CBU_1639 to $\mathrm{CBU}_{-} 1430$ & $\begin{array}{l}\text { AUR58_04175 to } \\
\text { AUR58_05380 }\end{array}$ & AUR58_04165 & AUR58_05390 \\
\hline & 7 & CBU_0343 to CBU_0446 & $\begin{array}{l}\text { AUR58_05410 to } \\
\text { AUR58_05920 }\end{array}$ & AUR58_05405 & AUR58_05930 \\
\hline & 8 & CBU_1369 to $\mathrm{CBU} \_1186 \mathrm{~b}$ & $\begin{array}{l}\text { AUR58_05960 to } \\
\text { AUR58_06965 }\end{array}$ & AUR58_05950 & AUR58_06975 \\
\hline & 9 & CBU_0620 to CBU_0714 & $\begin{array}{l}\text { AUR58_06980 to } \\
\text { AUR58_07480 }\end{array}$ & AUR58_06975 & AUR58_07485 ${ }^{b}$ \\
\hline & 10 & CBU_0722 to CBU_0716 & $\begin{array}{l}\text { AUR58_07490 to } \\
\text { AUR58_07535 }\end{array}$ & AUR58_07485 ${ }^{b}$ & AUR58_07540 ${ }^{b}$ \\
\hline & 11 & CBU_1085 to CBU_1063 & $\begin{array}{l}\text { AUR58_07545 to } \\
\text { AUR58_07675 }\end{array}$ & AUR58_07540 ${ }^{b}$ & AUR58_07685 \\
\hline & 12 & CBU_0758a to CBU_1061 & $\begin{array}{l}\text { AUR58_07695 to } \\
\text { AUR58_09285 }\end{array}$ & AUR58_07685 & AUR58_09295 \\
\hline & 13 & CBU_0758 to CBU_0724a & $\begin{array}{l}\text { AUR58_09300 to } \\
\text { AUR58_09475 }\end{array}$ & AUR58_09295 & AUR58_09480 ${ }^{b}$ \\
\hline & 14 & CBU_1087 to CBU_1185 & $\begin{array}{l}\text { AUR58_07485 to } \\
\text { AUR58_09975 }\end{array}$ & AUR58_09480 ${ }^{b}$ & AUR58_09985 \\
\hline & 15 & CBU_0619 to CBU_0600 & $\begin{array}{l}\text { AUR58_09995 to } \\
\text { AUR58_10110 }\end{array}$ & AUR58_09985 & AUR58_10125 ${ }^{c}$ \\
\hline & 16 & CBU_0580 to CBU_0599 & $\begin{array}{l}\text { AUR58_10130 to } \\
\text { AUR58_10225 }\end{array}$ & AUR58_10125 $5^{c}$ & AUR58_10230 ${ }^{b}$ \\
\hline & 17 & CBU_0579 to CBU_0447 & $\begin{array}{l}\text { AUR58_10240 to } \\
\text { AUR58_10935 }\end{array}$ & AUR58_10230 ${ }^{b}$ & AUR58_10945 \\
\hline & 18 & CBU_1372 to CBU_1427 & $\begin{array}{l}\text { AUR58_10950 to } \\
\text { AUR58_11235 }\end{array}$ & AUR58_10945 & AUR58_11240 \\
\hline & 19 & CBU_0341 to CBU_0274 & $\begin{array}{l}\text { AUR58_11265 to } \\
\text { AUR58_11615 }\end{array}$ & AUR58_11255 & AUR58_11620 \\
\hline \multirow[t]{4}{*}{ Z3055 } & 1 & $\begin{array}{l}\text { TY29_RS01425 to } \\
\text { TY29_RS00720 }\end{array}$ & $\begin{array}{l}\text { AUR58_00225 to } \\
\text { AUR58_00970 }\end{array}$ & AUR58_11620 ${ }^{b}$ & None $^{a}$ \\
\hline & 2 & $\begin{array}{l}\text { TY29_RS00715 to } \\
\text { TY29_RS00225 }\end{array}$ & $\begin{array}{l}\text { AUR58_00985 to } \\
\text { AUR58_01480 }\end{array}$ & None $^{a}$ & AUR58_01490 \\
\hline & 3 & $\begin{array}{l}\text { TY29_RS09935 to } \\
\text { TY29_RS10590 }\end{array}$ & $\begin{array}{l}\text { AUR58_01495 to } \\
\text { AUR58 } 02180\end{array}$ & AUR58_01490 & AUR58_02185 \\
\hline & 4 & $\begin{array}{l}\text { TY29_RS00005 to } \\
\text { TY29_RS00210 }\end{array}$ & $\begin{array}{l}\text { AUR58_02195 to } \\
\text { AUR58_02400 }\end{array}$ & AUR58_02185 & AUR58_02405 \\
\hline
\end{tabular}




\begin{tabular}{|c|c|c|c|c|}
\hline 5 & $\begin{array}{l}\text { TY29_RS09920 to } \\
\text { TY29_RS08410 }\end{array}$ & $\begin{array}{l}\text { AUR58_02415 to } \\
\text { AUR58_04055 }\end{array}$ & AUR58_02405 & AUR58_04065 \\
\hline 6 & $\begin{array}{l}\text { TY29_RS08335 to } \\
\text { TY29_RS08405 }\end{array}$ & $\begin{array}{l}\text { AUR58_04075 to } \\
\text { AUR58_04160 }\end{array}$ & AUR58_04065 & AUR58_04165 \\
\hline 7 & $\begin{array}{l}\text { TY29_RS08315 to } \\
\text { TY29_RS07895 }\end{array}$ & $\begin{array}{l}\text { AUR58_04175 to } \\
\text { AUR58_04650 }\end{array}$ & AUR58_04165 & None $^{a}$ \\
\hline 8 & $\begin{array}{l}\text { TY29_RS07885 to } \\
\text { TY29_RS07280 }\end{array}$ & $\begin{array}{l}\text { AUR58_04660 to } \\
\text { AUR58_05380 }\end{array}$ & None $^{a}$ & AUR58_05390 \\
\hline 9 & $\begin{array}{l}\text { TY29_RS01745 to } \\
\text { TY29_RS02250 }\end{array}$ & $\begin{array}{l}\text { AUR58_05410 to } \\
\text { AUR58_05920 }\end{array}$ & AUR58_05405 & AUR58_05930 \\
\hline 10 & $\begin{array}{l}\text { TY29_RS06980 to } \\
\text { TY29_RS06040 }\end{array}$ & $\begin{array}{l}\text { AUR58_05960 to } \\
\text { AUR58_06965 }\end{array}$ & AUR58_05950 & AUR58_06975 \\
\hline 11 & $\begin{array}{l}\text { TY29_RS03130 to } \\
\text { TY29_RS03620 }\end{array}$ & $\begin{array}{l}\text { AUR58_06980 to } \\
\text { AUR58_07480 }\end{array}$ & AUR58_06975 & AUR58_07485 \\
\hline 12 & $\begin{array}{l}\text { TY29_RS03665 to } \\
\text { TY29_RS03635 }\end{array}$ & $\begin{array}{l}\text { AUR58_07490 to } \\
\text { AUR58_07535 }\end{array}$ & AUR58_07485 $5^{b}$ & AUR58_07540 \\
\hline 13 & $\begin{array}{l}\text { TY29_RS05525 to } \\
\text { TY29_RS05405 }\end{array}$ & $\begin{array}{l}\text { AUR58_07545 to } \\
\text { AUR58_07675 }\end{array}$ & AUR58_07540 ${ }^{b}$ & AUR58_07685 \\
\hline 14 & $\begin{array}{l}\text { TY29_RS03850 to } \\
\text { TY29_RS05385 }\end{array}$ & $\begin{array}{l}\text { AUR58_07695 to } \\
\text { AUR58_09285 }\end{array}$ & AUR58_07685 & AUR58_09295 \\
\hline 15 & $\begin{array}{l}\text { TY29_RS03845 to } \\
\text { TY29_RS03675 }\end{array}$ & $\begin{array}{l}\text { AUR58_09300 to } \\
\text { AUR58_09475 }\end{array}$ & AUR58_09295 & AUR58_09480 \\
\hline 16 & $\begin{array}{l}\text { TY29_RS05540 to } \\
\text { TY29_RS06020 }\end{array}$ & $\begin{array}{l}\text { AUR58_07485 to } \\
\text { AUR58_09975 }\end{array}$ & AUR58_09480 ${ }^{b}$ & AUR58_09985 \\
\hline 17 & $\begin{array}{l}\text { TY29_RS03125 to } \\
\text { TY29_RS03035 }\end{array}$ & $\begin{array}{l}\text { AUR58_09995 to } \\
\text { AUR58_10110 }\end{array}$ & AUR58_09985 & AUR58_10125 \\
\hline 18 & $\begin{array}{l}\text { TY29_RS02935 to } \\
\text { TY29_RS03030 }\end{array}$ & $\begin{array}{l}\text { AUR58_10130 to } \\
\text { AUR58_10225 }\end{array}$ & AUR58_10125 ${ }^{c}$ & AUR58_10230 \\
\hline 19 & $\begin{array}{l}\text { TY29_RS02930 to } \\
\text { TY29_RS02255 }\end{array}$ & $\begin{array}{l}\text { AUR58_10240 to } \\
\text { AUR58_10935 }\end{array}$ & AUR58_10230 ${ }^{b}$ & AUR58_10945 \\
\hline 20 & $\begin{array}{l}\text { TY29_RS06995 to } \\
\text { TY29_RS07270 }\end{array}$ & $\begin{array}{l}\text { AUR58_10950 to } \\
\text { AUR58_11235 }\end{array}$ & AUR58_10945 & AUR58_11240 \\
\hline 21 & $\begin{array}{l}\text { TY29_RS01740 to } \\
\text { TY29_RS01390 }\end{array}$ & $\begin{array}{l}\text { AUR58_11265 to } \\
\text { AUR58 } 11615\end{array}$ & AUR58_11255 & AUR58_11620 \\
\hline
\end{tabular}





\section{Chapter 6}

\section{Coxiella burnetii strain specific}

immune responses are more

\section{depending on host origin than on MLVA genotype classification}

Runa Kuley, Hendrik I. J. Roest, Mari A. Smits, Ad P. Koets, Hilde E. Smith and Alex Bossers 


\begin{abstract}
Q fever is a zoonotic disease caused by the intracellular replicating bacterium Coxiella burnetii. Domestic ruminants such as goats, sheep and cattle are the main hosts of $C$. burnetii causing infections in humans. Clinical symptoms of Q fever like abortion are commonly seen in goats and rarely in cattle. Human Q fever outbreaks are frequently associated with goats or sheep and only a few cases are attributed to cattle as the source of infection. The major Q fever outbreak in The Netherlands during 2007-2010 underlined this association, since the C. burnetii genotype (CbNL01) that is mainly associated with small-ruminants, was linked to human cases, in contrast to the genotype (CbNL12) that is predominant in cattle. In the present study, we investigated innate and adaptive immune responses against $C$. burnetii infection in an in vivo mice model to assess whether differences in immune responses to strains from different host-origin could be an explanation of the divergent clinical outcomes in cattle, goats and humans. At several time points after infection of mice with $C$. burnetii strains, we measured specific phase I and II antibody levels, mRNA levels of several cytokines-chemokines in splenocytes, and the number of IFN- $\gamma$ producing cells upon stimulation of peritoneal cells. Differences in immune responses were dependent on the host-origin of the C. burnetii strains and not on the MLVA genotype of the strains. Among the different strains, the cattle-derived strains generated a significantly higher IgG antibody level against phase I antigen, a higher number of IFN- $\gamma$ producing cells, as well as significantly higher levels of pro-inflammatory cytokines and chemokines mRNA. Compared to goat and human-derived strains, the cattle strains induced a more profound inflammatory and protective immune response. Such a strong immune response against cattle-derived strains may explain the low incidence of $C$. burnetii induced abortions in cattle and the absence of clear clinical signs of $C$. burnetii infections in cattle. On the other hand, the inadequate induction of immune response of mice against goat-derived strains may be indicative for the increased risks of the transmission of $\mathrm{Q}$ fever from goats to humans. Additionally, similar immune responses generated by goat and human strains might indicate similar virulence mechanisms adapted by these strains in host.
\end{abstract}




\section{Introduction}

C. burnetii is the causative agent of $\mathrm{Q}$ fever and infects a wide range of hosts. Among these, infections are wide-spread in domestic ruminants such as goats, sheep and cattle which are the primary reservoirs of the disease [1-3]. C. burnetii infections in pregnant goats commonly cause abortion [4,5], whereas infected cattle usually do not show disease symptoms. Abortions and/or reproductive disorders like metritis are rarely manifested in cattle [5-7]. Q fever is a zoonotic disease and infections of humans are most often caused by inhalation of contaminated aerosols, which can lead to an acute or chronic infection [1,5,8-10]. The zoonotic potential of the bacterium has been underlined in the $\mathrm{Q}$ fever outbreak during the years 2007-2010 in The Netherlands. During this epidemic, more than 4000 human cases have been reported and around 40,000 were assumed to be infected based on seroprevalence studies $[6,11,12]$. Genotyping studies showed the predominant presence of a single unique genotype in all strains obtained from goats and human patients. This identified goats as the major source of the Dutch human Q fever outbreak [6,13]

Most of the Q fever outbreaks reported so far predominantly involved goats and sheep as a source of human infections and only sporadic cases have been attributed to cattle $[5,6,14,15]$. On the contrary, seroprevalence studies in different European countries, including The Netherlands, showed higher prevalence of $C$. burnetii specific antibodies in cattle herds as compared to goats and sheep herds $[15,16]$. Several studies have also shown that cattle strains are associated with specific genotypes, which are different from the genotype that is predominantly found in human cases $[6,17,18]$. During the Dutch outbreak, C. burnetii was found in a majority of the dairy cattle herds. However, samples from cattle were genotyped as CbNL12 and were clearly distinct from the genotype of the Dutch outbreak strains [6,19]. This predominant cattle-associated MLVA genotype was rarely detected in goats and humans $[6,20]$. Therefore, despite being a huge reservoir of $C$. burnetii, cattle herds probably pose only a low risk to humans since human Q fever infections/ outbreaks are sporadically related to cattle-associated genotypes. The reduced risk of the $C$. burnetii reservoir in cattle, as compared to goat, could be due to a low susceptibility of cattle to develop clinical Q fever and a concomitant reduction of bacterial shedding in the environment [4,21]. During the large Dutch Q fever outbreak, C. burnetii was detected in $60 \%$ of dairy goat farms with abortive problems [6], whereas only few aborted placentas from cow's were positive for C. burnetii. During abortions in goats, large amounts of $C$. burnetii are shed into the environment and are the main source of infection in humans. Besides these factors, goats but not cattle are seasonal breeders which might limit the exposure of humans to cattle-derived strains [22,23]. Other factors contributing to the low risk of cattle strains to cause Q fever in humans could also be attributed to the genetic potential of cattlespecific $C$. burnetii strains. Cattle strains differ genotypically from the majority of strains that could be isolated from goat and human and could potentially be less virulent in humans, resulting in no/ mild/ sub-clinical forms of the infectious disease. The contribution of these CbNL12 goat strains in the Dutch outbreak are not completely understood yet. Although several factors may contribute to 
different host-ranges and/or disease pathology of the various C. burnetii strains, the exact underlying mechanisms are largely unknown. A possible mechanism could be based on C. burnetii strain specific differences in antigenic potential and the type of immune responses they induce.

During the Dutch outbreak period, we were successful in isolating and culturing several $C$. burnetii strains of CbNL01 genotype, isolated from goat and humans, CbNL12 genotype goat strains and the cattle related CbNL12 MLVA genotype strains. It is not known yet whether the host-origin of the $C$. burnetii strain or the MLVA genotype is the primary determinant to direct immune responses in infected hosts. This is important to know, as it may imply that it is more important to monitor the hostorigin of $C$. burnetii than to know its genotype, in order to improve the prevention of Q fever. Therefore, the objective of the present study was to investigate whether $C$. burnetii strains originating from various hosts (goats, cattle and humans) and having different MLVA genotypes induce different immune responses in an established murine model. Based upon the observed low risk of cattle strains in Q fever outbreaks and the mild/no clinical symptoms generally noticed in natural cattle hosts, we hypothesized that cattle strains are effectively recognized by the host immune system and induce a protective immunity, which results in an effective bacterial clearance and the development of no or only mild forms of the disease. To this end, we measured phase I and II IgM and IgG antibody levels in blood, the number of IFN- $\gamma$ producing cells upon stimulation of peritoneal cells isolated from infected mice, and the mRNA levels of several pro-inflammatory cytokines/chemokines in splenocytes as indicators of adaptive and innate immune responses. Both types of responses are known to be necessary to limit intracellular bacterial infections and both were demonstrated to be important in limiting C. burnetii infections in mice [18,24,25]. Our results demonstrate that the cattle-derived strains induced significantly higher innate and adaptive immune responses in mice as compared to goat and human-derived strains. This indicates that the origin of C. burnetii strains has a greater impact on immune responses in mice, rather than the MLVA genotype of the C. burnetii strains.

\section{Material and methods}

\section{C. burnetii strains}

The C. burnetii strains X09003262-001 (3262), CbCVIC1 and 8014160-001 (601) were primarily isolated from aborted goats from different farms during the Dutch Q fever outbreak (2007-2010). The human strains 42785537 and 3345937 were isolated from heart valve of two individual chronic Q fever patients from Radboud University Medical Center and Canisius Wilhelmina hospital respectively from The Netherlands [6,26]. Strains CbG15D6 and 701CbB1 were obtained from goat and cattle aborted placentas from France and were kindly provided by A. Rodolakis (INRA, France). The cattle strain $\mathrm{CbBeB} 1$ was isolated from bulk milk in Belgium and was kindly provided by D. Fretin (Coda Cerva, Brussels) [18]. The other human strains Henzerling and Scurry were kindly provided by D. Frangoulidis (Bundeswehr Institute of Microbiology, Munich). All strains were typed using Multiple Locus Variable number of tandem repeat Analysis (MLVA) and were initially cultured 
in BGM cells (European Collection of Cell Cultures, Salisbury, UK) [27,28]. The characteristics of the strains are indicated in Table 1. The stock cultures of all the strains cultured from BGM cells were stored at $-80^{\circ} \mathrm{C}$. Prior to experimentally infecting mice, the strains were cultured once in BGM cells and freeze thawed to obtain bacterial cells. Low passages of these strains (between 4-14 passages) were maintained to avoid, as much as possible, the phase variation. The cultured strains were quantified by PMA-PCR to obtain live amounts of bacteria as described previously [28]. All strains represented phase I protein profiles as analyzed by SDS-PAGE followed by silver staining, using the bacterial whole cell lysates as described previously [29-31]. Purified phase I and Phase II NM LPS kindly provided by R. Toman (Institute of Virology, Slovakia) were used as controls.

Table 1. C. burnetii strains used in this study

\begin{tabular}{lllllll}
\hline Strain & Genotype & Country & Host & Source & Symptom & Reference \\
\hline 3262 & CbNL01 & Netherlands & Goat & Placenta & Abortion & {$[19]$} \\
CbCVIC1 & CbNL01 & Netherlands & Goat & Placenta & Abortion & This study \\
601 & CbNL12 & Netherlands & Goat & Placenta & Abortion & {$[19]$} \\
CbG15D6 & CbNL12 & France & Goat & Placenta & Abortion & This study \\
701 CbB1 & CbNL12 & France & Cattle & Placenta & Abortion & {$[19]$} \\
CbBEB1 & CbNL12 & Belgium & Cattle & Bulk milk & - & {$[18]$} \\
3345937 & CbNL01 & Netherlands & Human & heart valve & Endocarditis & {$[19]$} \\
42785537 & CbNL01 & Netherlands & Human & heart valve & Endocarditis & This study \\
Henzerling & Henzerling & Italy & Human & Blood & Acute & {$[19]$} \\
Scurry & Scurry & USA & Human & Liver & Hepatitis & {$[19]$} \\
\hline
\end{tabular}

\section{Murine infection model}

Animal experiments were conducted using 7 week old Specific-Pathogen-Free Swiss female OF1 mice (Charles River, l'Arbresle, France). A total of 88 mice were housed under sterile conditions in biosafety level 3 facilities and acclimatized for a week before experimental infection. Eight mice per experimental group were injected intraperitoneally with $8 \times 10^{4}$ viable C. burnetii strain (in $200 \mu 1 \mathrm{PBS}$ ) as measured by PMA-PCR. A group of 8 control mice were injected with PBS only. Two mice from each experimental group were sacrificed at each time point (7, 14, 21 and 28 days post infection (p.i.)). Blood, spleen and peritoneal cells were collected from sacrificed mice for further analysis. Mice were weighed and their spleens were aseptically removed, weighed and immediately frozen in liquid nitrogen and stored at $-80^{\circ} \mathrm{C}$ in order to preserve RNA. These weights were used for determining relative spleen weights (percentage of means of spleen weight/body weight). Bacterial loads of spleens were measured as described previously [28].

Animal experiments were approved by the animal experiment commission of Wageningen Bioveterinary Research, and were conducted in accordance with the Dutch regulations on animal 
experimentation (Registration numbers: 2014013). Humane end points of the experiments were defined prior to the actual experiments and all possible measures were taken to minimize animal suffering.

\section{Detection and quantification of phase I and II IgM and IgG antibodies}

C. burnetii specific phase I and II IgM and IgG antibody levels were quantified by adaptation of the commercially available phase I and II specific ELISA kit from Virion/ Serion (SERION ELISA classic Coxiella burnetii Phase 1 and 2, Germany). Optimal serum and anti-mouse IgG and IgM conjugate dilutions were assessed in advance based on two-way titrations conducted on both positive and negative samples originating from infected and uninfected animals respectively. Maximal absorbance within the linear range occurred with a mouse serum dilution of 1:200 and all further analyses were performed using this dilution (data not shown). Plates containing specific phase I and II antigens were incubated with $100 \mu \mathrm{l}$ of 1:200 diluted serum in dilution buffer provided in the kit for an hour at $37^{\circ} \mathrm{C}$. For phase I and II IgM and IgG quantification, alkaline phosphatase conjugated goat anti-mouse IgM mu chain antibody and rabbit anti-mouse IgG H\&L antibody (Abcam, Cambridge, USA) was found to perform best at 1:5000 and 1:1000 dilutions respectively. All other steps were conducted according to manufacturer's instructions. The optical density was measured at $405 \mathrm{~nm}$ (EL 808 Ultra microplate reader, Bio-tek instruments, USA). Results are presented based on optical density values from serum samples of infected mice normalized with values from uninfected control mice [18]. The normalized antibody titers were used for comparing humoral immunity generated in mice by $C$. burnetii strains originating from different host-origin or MLVA genotypes.

\section{Interferon- $\gamma$ Enzyme-linked immunosorbent spot (ELISPOT) assay}

IFN- $\gamma$ ELISPOT assay was performed using a kit from eBioscience as per the manufacturer's specifications. Briefly, we pre-coated ELISPOT plates (Millipore 96-well MultiScreen HTS) with the capture antibody at $4^{\circ} \mathrm{C}$ overnight. Unbound antibody was removed by washing with PBS. Prior to the experiments, plates were blocked with complete media (RPMI 1640, Invitrogen, supplemented with $10 \%$ FCS, 1\% L-glutamine, 0.1\% BME and 1\% penicillin/streptomycin) for $30 \mathrm{~min}$ at room temperature. At each time point mouse peritoneal cells were isolated from sacrificed infected and control mice by washing the peritoneal cavity with $5 \mathrm{ml}$ of cold PBS. The cells obtained were centrifuged at $1700 \mathrm{RPM}$ for $5 \mathrm{~min}$ at $4^{\circ} \mathrm{C}$ and washed twice with complete media. The cells were counted with a Scepter cell counter (Millipore), supplied with a $40 \mu \mathrm{m}$ sensor and were subsequently plated at a density of $2 \times 10^{5}$ cells/well from each mouse. The peritoneal cells were stimulated with 1:2000 dilution of C. burnetii Nine Mile phase I and II antigens (Virion/ Serion, Germany) separately. Concanavalin A was used as a positive control and complete media was used as a negative control. The cells were incubated overnight at $37^{\circ} \mathrm{C}$ and $5 \% \mathrm{CO} 2$. To detect the spots, the plates were washed and incubated with biotin-conjugated secondary antibody for 2 hours at room temperature or $4^{\circ} \mathrm{C}$ 
overnight. The spots were developed with Avidin-HRP and peroxidase substrate AEC (3-amino-9ethyl carbazole, Sigma). Plates were dried in a dry incubator at $37^{\circ} \mathrm{C}$. Spots were counted with an ImmunoSpot analyzer (CTL, USA). We optimized the counting parameters and the number of specific spots was determined as the average of duplicates from each sample after the average number of the respective negative controls was subtracted. This corrected number of spots was used for comparing cell mediated immunity in response to strains originating from different host-origin or MLVA genotypes.

\section{Cytokine and chemokine mRNA measurements}

For determining cytokine and chemokine mRNA levels we used a microarray approach (Mice microarray (G4852A), Agilent Technologies) to measure the expression levels of cytokine and chemokine producing genes [32]. Total RNA was isolated from mice spleen by using Direct-zol RNA Miniprep Kit (Zymo Research, Irvine, USA) as per manufacturer's instructions. The microarray experiments and data analysis were performed as described previously $[28,33]$. The average gene expression of cytokine and chemokine genes of controls and infected mice was normalized with expression of the housekeeping gene ACTB [34]. These normalized values were used for comparing cytokine and chemokine levels generated in mice exposed to the strains that originated from different host-origin or MLVA genotypes.

\section{Statistical analysis}

Data for the parameters antibody assay, ELISPOT assay and cytokine-chemokine levels from mouse experiments were calculated by grouping $C$. burnetii strains based on their host-origin and genotype and were expressed as means \pm standard error of mean. The bacterial load in spleen was expressed as means \pm standard deviation. Statistical significant differences in a parameter for different groups were assessed by using the Linear Mixed Model to test significant host-origin*genotype interactions at 7 , 14, 21 and 28 days p.i. time points. This was followed by Fisher LSD Post Hoc tests to assess differences in groups with a significant interaction. The Linear Mixed Model was also used to assess significant differences in the rate of change over time (slope) of a parameter for host-origin*over time interaction. Further, $t$ tests were used, for determining differences in a group over time with a significant interaction. $t$ tests were also used to determine significant differences in a parameter per time point between controls and cattle, goat, human strain inoculated groups of mice. All statistical tests were performed using IBM SPSS 20 software and differences were considered significant at a $P$ value of $<0.05$. 


\section{Results}

In the present study, innate and adaptive immune responses generated by mice upon infection of various $C$. burnetii strains were measured. Successful infections of mice by $C$. burnetii strains were detected based on significant levels of splenomegaly in infected animals compared to the respective controls $(p<0.05$, data not shown). All the immune parameters were calculated by grouping strains based on their host-origin (goat, cattle and human) or MLVA genotype (CbNL01, CbNL12 and others) as given in Table 1. All the various immune parameters were measured as indicated in Material and Methods and significant host-origin*genotype interactions were calculated for the various time points by multilevel statistics. The results are presented in Table 2 and are explained in detail below.

Table 2. A Significant difference matrix indicating host-origin*genotype interaction of C. burnetii strains grouped on their host-origin and genotype at different time points on various immune response parameters

\begin{tabular}{|c|c|c|c|c|c|c|c|c|c|}
\hline \multirow{3}{*}{\multicolumn{2}{|c|}{ Immune response }} & \multicolumn{8}{|c|}{ Time-point } \\
\hline & & \multicolumn{2}{|r|}{7} & \multicolumn{2}{|c|}{14} & \multicolumn{2}{|c|}{21} & \multicolumn{2}{|r|}{28} \\
\hline & & $\begin{array}{l}\text { Host } \\
\text { origin }\end{array}$ & Genotype & $\begin{array}{l}\text { Host } \\
\text { origin }\end{array}$ & Genotype & $\begin{array}{l}\text { Host } \\
\text { origin }\end{array}$ & Genotype & $\begin{array}{l}\text { Host } \\
\text { origin }\end{array}$ & Genotype \\
\hline \multirow{4}{*}{ 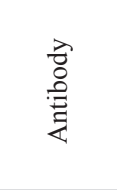 } & IgMp2 & - & - & - & - & - & - & - & - \\
\hline & IgGp2 & - & - & - & - & - & - & - & - \\
\hline & IgMp1 & - & - & - & - & - & - & - & - \\
\hline & IgGp1 & - & - & - & - & - & - & $*$ & - \\
\hline 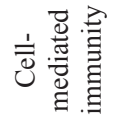 & $\begin{array}{l}\text { IFN } \gamma \\
\text { secreting } \\
\text { cells }\end{array}$ & - & - & - & - & - & - & $*$ & - \\
\hline \multirow{10}{*}{ 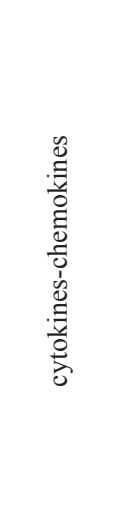 } & IFN-y & - & - & - & - & - & - & $*$ & - \\
\hline & TNF-a & - & - & - & - & $*$ & - & $*$ & - \\
\hline & IL-1b & - & - & - & - & - & - & $*$ & - \\
\hline & IL-6 & - & - & - & - & - & - & $*$ & - \\
\hline & IL-12a & - & - & - & - & $*$ & - & - & - \\
\hline & CCL2 & - & - & - & - & - & - & $*$ & - \\
\hline & CCL6 & - & - & - & - & $*$ & - & $*$ & - \\
\hline & CXCL10 & - & - & - & - & - & - & $*$ & - \\
\hline & IL-10 & - & - & - & - & $*$ & - & $*$ & - \\
\hline & IL-1RA & - & - & - & - & - & - & $*$ & - \\
\hline
\end{tabular}

Significant differences were indicated as $*(p<0.05)$ and non-significant differences were indicated as $-(p>$ $0.05)$ 


\section{Similar antibody responses by $\boldsymbol{C}$. burnetii strains of different origin}

Blood samples from infected and control mice were collected from sacrificed mice and the extracted serum was used to measure IgM and IgG phase I and II specific antibodies. From the multilevel analysis, an effect of $C$. burnetii host-origin as well as genotype group was not significant for all time points with regard to the IgM and IgG antibody levels against phase II antigens (IgMpII, IgGpII) and IgM antibody levels against phase I antigens (IgMpI) (Table 2). This indicates that these antibody levels per time point were similar in response to all C. burnetii strains originating from different hosts (Fig 1a-1c) or from different MLVA genotypes. However, statistically significant differences in kinetics of IgMpII, IgGpII and IgMpI antibody responses could be observed overtime in all strains
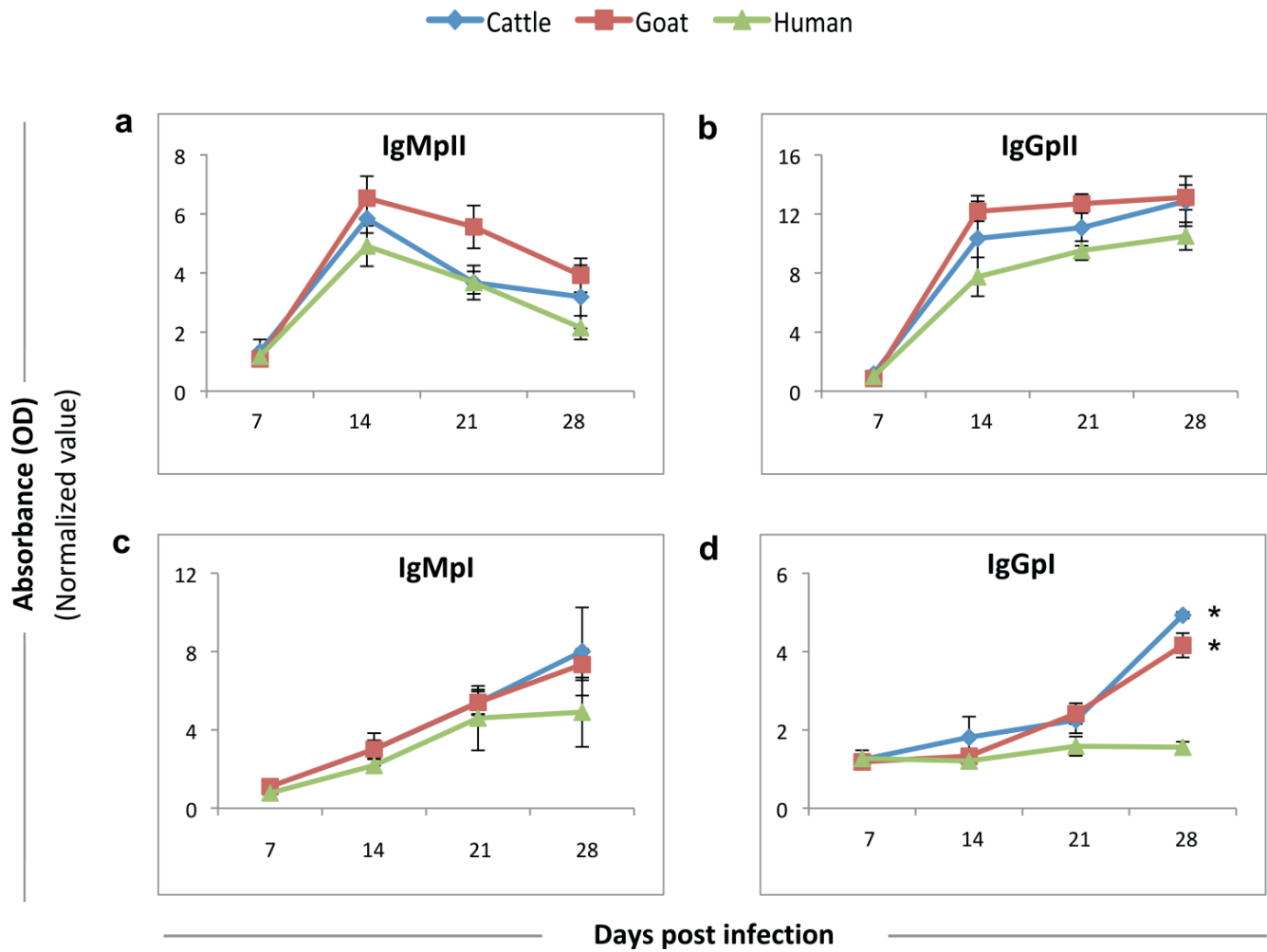

Figure 1. Mice Phase I and II specific IgM and IgG antibodies levels generated in response to C. burnetii strains grouped on their origin from cattle, goat and human hosts. The panels are grouped on average IgM and IgG antibody levels against $C$. burnetii strains of various host-origins at 7, 14, 21 and 28 day time points after inoculation of mice. a) IgM antibody against phase II (IgMpII) b) IgG antibody against phase II (IgGpII) c) IgM antibody against phase I (IgMpI) d) IgG antibody against phase I (IgGpI). Significant higher IgGpI antibody levels in response to cattle and goat-derived strains than to human-derived strains at 28 days p.i. are indicated by $*(p<0.05)$. 
grouped on their host-origin (Fig 1a, b and c). The average IgMpII level overtime peaks significantly at 14 days p.i. (data not shown) and then gradually decreases in response to goat, cattle and human strains (Fig 1a). The average IgGpII level also significantly increases overtime at 14 days p.i. (data not shown) and gradually increases till the end of the experiment (Fig 1b). With respect to antibody levels against phase I antigens, the average IgMpI levels significantly increases overtime (data not shown) during the whole experimental period (Fig 1c). Significant differences in the IgG antibody levels against phase I antigen (IgGpI) overtime were also observed. The IgGpI levels significantly increase at 28 days p.i. (data not shown) in response to goat and cattle strains, whereas no significant differences in IgGpI were observed at all time points (data not shown) in response to human strains (Fig 1d). From the multilevel analysis, a significant effect was seen in the host-origin group but not in the genotype group at 28 days p.i. on IgGpI antibody response (Table 2). These differences in host-origin strains at 28 day p.i. were due to significant low antibody responses to human strains compared to goat and cattle strains (Fig 1d). Thus, IgMpII, IgGpII and IgMpI kinetics as well as antibody titers were similar among the various host-origin groups. However, differences in IgGpI kinetics could be observed later in the infection period. Besides, no differences in antibody levels were found between the MLVA grouped strains (Table 2). This indicates that the profile of humoral immunity kinetics is determined by the host-origin of $C$. burnetii and not by its MLVA genotype.

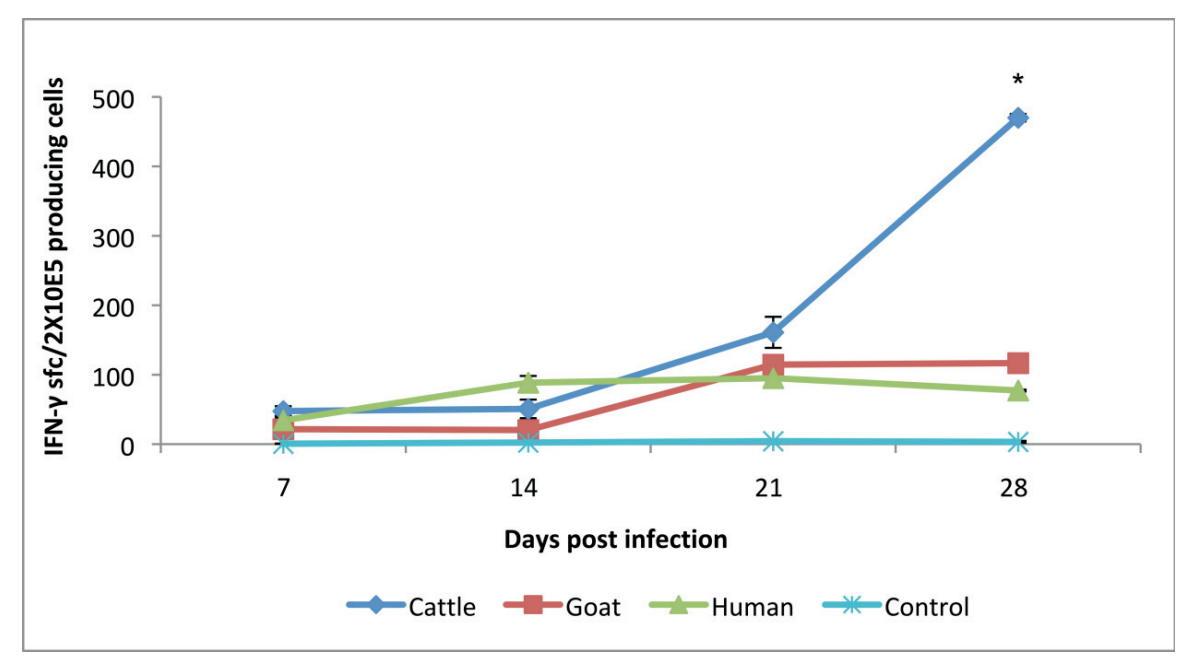

Figure 2. Detection of IFN- $\gamma$ producing peritoneal cells of mice infected with $C$. burnetii strains grouped on their origin from cattle, goat and human hosts.along with corresponding controls. The results are expressed as the mean number of spot forming cells (SFC) per $2 \times 10^{5}$ peritoneal cells stimulated with phase I and II antigens. Significant higher IFN- $\gamma$ producing peritoneal cells of mice infected with cattle-derived strains than goat and human-derived strains at 28 days p.i. were indicated as $*(p<0.05)$. 


\section{Cattle-derived strains generate a more profound cell mediated immune response}

IFN- $\gamma$ ELISPOT assays were performed on peritoneal cells of infected mice and corresponding controls to investigate cell mediated immune responses. Commercially obtained C. burnetii phase I and II antigens of reference Nine Mile strain were used as stimulus. The stimulants induced a clear IFN- $\gamma$ response in peritoneal cells from all infected mice compared to respective controls (Fig 2). The kinetics of the number of IFN- $\gamma$ producing peritoneal cells obtained from mice infected with goat, cattle and human strains, was similar at 7, 14 and 21 days. However, at day 28 the number of IFN- $\gamma$

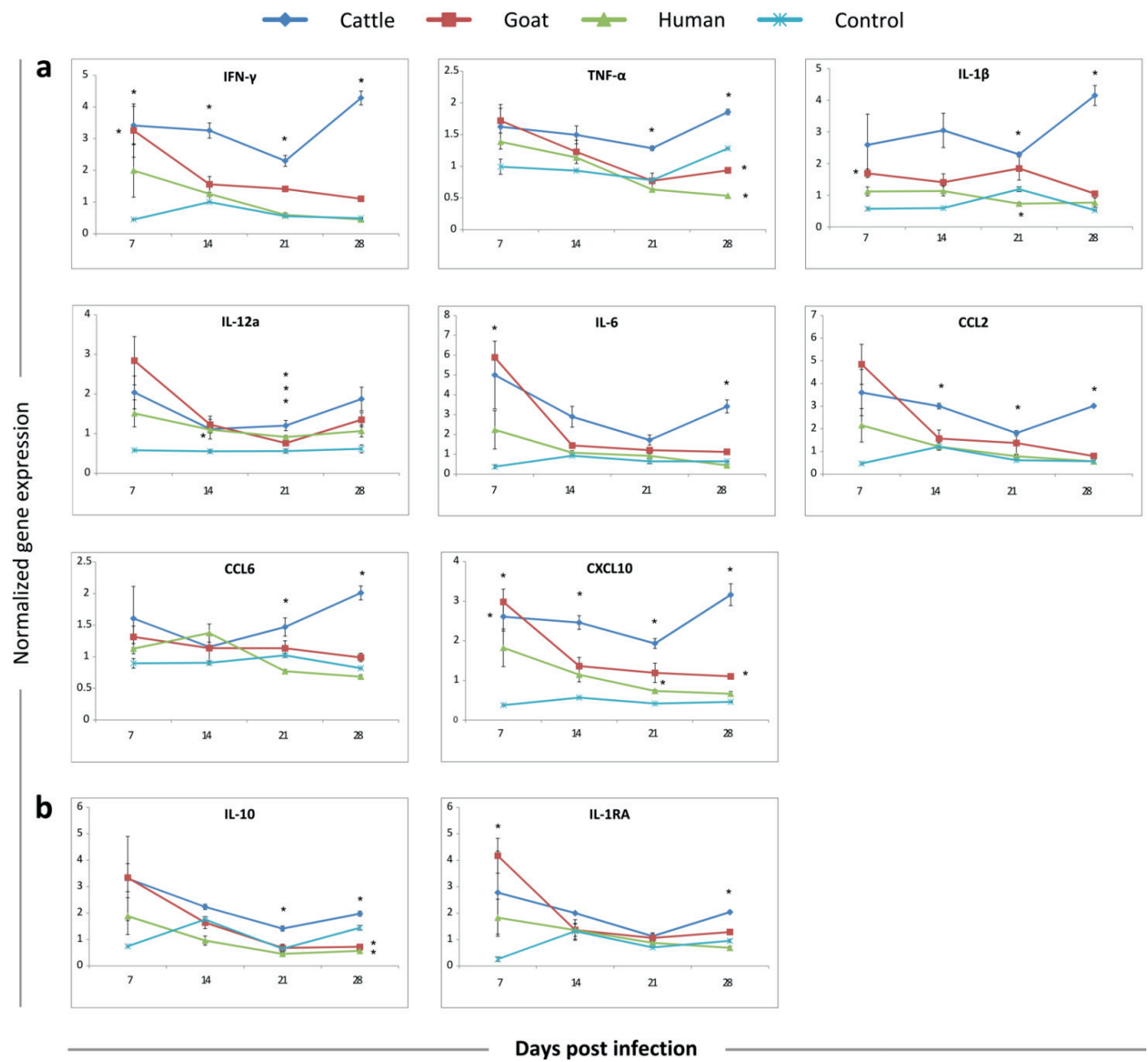

Figure 3. Normalized gene expression of cytokine and chemokine profiles in spleens of mice exposed to $C$. burnetii strains grouped on their origin and corresponding controls. The results show cytokine and chemokine mRNA levels after infection with C. bunetii of goat, cattle and human origin (color coded lines). Indicated are: a) pro-inflammatory cytokines and chemokines; and b) anti-inflammatory cytokines. Significant differences between infected mice and controls per time-point are indicated as $*(P<0.05)$. 
producing cells increased significantly only in peritoneal cells of mice infected with cattle origin strains compared to goat and human origin strains (Fig 2). From the multilevel analysis, a significant effect of $C$. burnetii host-origin but not of the genotype group was observed at 28 day p.i. in number of IFN- $\gamma$ producing peritoneal cells (Table 2). These results demonstrate that differences in cell mediated immunity are based on C. burnetii strains host-origin rather than on MLVA genotype.

\section{Cattle-derived strains induce a profound pro-inflammatory response}

To extend our observations on the immune response differences of $C$. burnetii strains, weekly measurements of mRNA expression of cytokine and chemokine genes were assessed in splenocytes. The results of these measurements are shown in Figure 3 and the significance of their relationship with host-origin*genotype interactions are shown in Table 2 . These involve the pro-inflammatory cytokines (IFN- $\gamma$, TNF- $\alpha$, IL-1 $\beta$, IL-6, IL-12a), inflammatory associated chemokines (CCL2, CCL6, CXCL10) (Fig 3a) and anti-inflammatory cytokines (IL-10, IL-1RA) (Fig 3b). The cytokine and chemokine mRNA levels in response to the cattle strains were in most cases significantly higher than the response to goat and human strains at all time points. In goat strains, the cytokine and chemokine mRNA levels were significantly higher than the controls only at early time points (7 day p.i.). The cytokine and chemokine mRNA levels in response to the human strains were higher than the controls at early time points (7 day p.i.), but they were mostly not significant (Fig 3). The kinetics of mRNA levels were similar in response to goat and human strains, with increased cytokine and chemokine levels early in

Table 3. A significant difference matrix of cytokine and chemokine mRNA levels generated by $C$. burnetii strains originating from goat, cattle and human strains at different time points $(7,14,21$ and 28 days p.i.). Significant differences were indicated as $*(p<0.05)$

\begin{tabular}{|c|c|c|c|c|c|c|c|c|c|c|c|c|}
\hline \multirow{3}{*}{$\begin{array}{l}\text { Cytokine/ } \\
\text { chemokines }\end{array}$} & \multicolumn{12}{|c|}{ mRNA levels comparisons between host-origin strains at 7, 14, 21 and 28 day time points } \\
\hline & $\begin{array}{l}\text { Cattle } \\
\text { vs } \\
\text { Goat }\end{array}$ & $\begin{array}{l}\text { Cattle } \\
\text { vs } \\
\text { Human }\end{array}$ & $\begin{array}{l}\text { Goat } \\
\text { vs } \\
\text { Human }\end{array}$ & $\begin{array}{l}\text { Cattle } \\
\text { vs } \\
\text { Goat }\end{array}$ & $\begin{array}{l}\text { Cattle } \\
\text { vs } \\
\text { Human }\end{array}$ & $\begin{array}{l}\text { Goat } \\
\text { vs } \\
\text { Human }\end{array}$ & $\begin{array}{l}\text { Cattle } \\
\text { vs } \\
\text { Goat }\end{array}$ & $\begin{array}{l}\text { Cattle } \\
\text { vs } \\
\text { Human }\end{array}$ & $\begin{array}{l}\text { Goat } \\
\text { vs } \\
\text { Human }\end{array}$ & $\begin{array}{l}\text { Cattle } \\
\text { vs } \\
\text { Goat }\end{array}$ & $\begin{array}{l}\text { Cattle } \\
\text { vs } \\
\text { Human }\end{array}$ & $\begin{array}{l}\text { Goat } \\
\text { vs } \\
\text { Human }\end{array}$ \\
\hline & 7 & 7 & 7 & 14 & 14 & 14 & 21 & 21 & 21 & 28 & 28 & 28 \\
\hline IFN- $\gamma$ & - & - & - & - & - & - & - & - & - & $*$ & $*$ & - \\
\hline TNF- $\alpha$ & - & - & - & - & - & - & * & $*$ & - & $*$ & $*$ & $*$ \\
\hline Il-1 $\beta$ & - & - & - & - & - & - & - & - & - & $*$ & $*$ & - \\
\hline IL-6 & - & - & - & - & - & - & - & - & - & $*$ & $*$ & - \\
\hline IL-12a & - & - & - & - & - & - & $*$ & - & - & - & - & - \\
\hline CCL2 & - & - & - & - & - & - & - & - & - & $*$ & $*$ & - \\
\hline CCL6 & - & - & - & - & - & - & - & $*$ & - & $*$ & $*$ & - \\
\hline CXCL10 & - & - & - & - & - & - & - & - & - & $*$ & $*$ & - \\
\hline IL-10 & - & - & - & - & - & - & $*$ & $*$ & - & $*$ & $*$ & - \\
\hline IL-1RA & - & - & - & - & - & - & - & - & - & $*$ & $*$ & $*$ \\
\hline
\end{tabular}


the infection period (7 day p.i.) and declining levels later in the infection. In contrast, the cytokine and chemokine response to cattle strains did not show large variations in time at 7, 14, and 21 days after infection, but increased significantly at 28 days p.i. (Fig 3). From the multilevel analysis, a significant effect of C. burnetii host-origin but not of the genotype group was observed at 21 and 28 day p.i. in cytokine-chemokine levels (Table 2). These differences in host-origin strains at 21 and 28 day p.i. were due to significantly higher cytokine and chemokine mRNA levels in response to cattle strains than goat and human strains (Table 3). The mRNA levels in response to goat strains were also significant higher for TNF- $\alpha$ and IL-1RA cytokines compared to the response to human strain (Table 3). Taken together, persistent higher cytokine and chemokine mRNA levels were observed in mice splenocytes in response to cattle-derived strains than in response to goat and human derived strains. This indicates that only cattle strains generate a profound pro- and anti-inflammatory response upon infection. In addition, the cytokine-chemokine responses did not change in mice infected by strains grouped on the basis of their MLVA genotype (Table 2). This suggests that innate immune responses are mainly driven by the host-origin of $C$. burnetii strains and not by their MLVA genotype.

\section{Discussion}

Several studies have suggested a genomic group-specific virulence of C. burnetii, based on the assumption that genetic differences in bacterial strains could affect its virulence. These studies involved strains mostly from human origin and a few from animal origin [35-38]. Our study involves the comparison of immune responses of a variety of strains from goat, cattle and human origin including those isolated from The Netherlands during the large outbreak period from 2007-2010. We compared the innate (cytokines and chemokines) and adaptive (humoral and cell-mediated) immune responses generated by these strains in a murine infection model. Our study clearly shows that the host-origin of the various $C$. burnetii strains, rather than the MLVA genotype, determines the outcome of the induced immune responses (Table 2). Significant interactions in host-origin strains were mostly due to significant differences in immune responses to cattle-derived strains as compared to goat and human-derived strains. These results suggest that host immune responses are more dependent on the host-origin of C. burnetii strains than on the MLVA genotype.

In mice, humoral antibody responses were similarly generated by cattle and goat strains at all tested time points, except those to the human strains where low $\operatorname{IgG}$ antibody response against phase I antigens (IgGpI) were observed at 28 day p.i. These low IgGpI antibody titers in response to human strains were not expected as whole cell bacterial lysates of all C. burnetii strains showed a protein profile typically for the presence of phase I LPS (data not shown). Although, all strains have a phase I type LPS, the low IgGpI levels raised by the human-derived strains could be due to a modified outer shell (LPS or surface proteins) with a reduced antigenicity. No significant difference in IgM and IgG antibody levels against phase I and II antigens were generated by strains of different genotypes. Hence, humoral immune responses seem to be dependent more on host-origin than on MLVA 
genotype of $C$. burnetii strains. Our results also showed early and strong systemic antibody response to goat and cattle origin strains of C. burnetii (Fig 1) as seen previously [18]. Also, the observed kinetics of IgM and IgG antibody's in mice against phase I and II antigens are in line with previous published work in goats and humans, strengthening mice as a valid experimental model for studying immune responses induced by $\mathrm{Q}$ fever agents $[39,40]$.

A significant effect of $C$. burnetii host-origin but not the genotype group was observed in cellmediated immune response due to augmented pro-inflammatory IFN- $\gamma$ cytokine production from peritoneal cells, especially at the later infection period (28 day p.i). IFN- $\gamma$ is an important cytokine produced by T-lymphocytes which is responsible for cell-mediated immunity [41,42]. During microbial infection, T-lymphocytes are found at sites of infection and implement cell-mediated immunity in response to bacterial antigens [43]. Isolated peritoneal cells from infected mice showed a significant higher IFN- $\gamma$ response upon stimulation with $C$. burnetii antigens than corresponding noninfected control mice. This could be due to the presence of a different composition of IFN- $\gamma$ secreting immune cells (T-lymphocytes, (natural killer) NK, NK T cells) [42] in the peritoneal cavity of infected mice due to intraperitoneal inoculation of bacteria. With respect to infection of $C$. burnetii strains from different origin, a similar kinetics of increase in IFN- $\gamma$ production was observed from initial to late infection period. This is most likely due to an influx of T-lymphocytes and other IFN- $\gamma$ producing cells in the peritoneal cavity as the infection progresses. The observed significant higher IFN- $\gamma$ response from peritoneal cells of mice infected with cattle strains compared to goat and human strains at 28 day p.i. period (Fig 2) is probably due to a larger number of pro-inflammatory T-lymphocytes in the peritoneal cavity. On the other hand, it is also possible that the low IFN- $\gamma$ response of peritoneal cells of mice infected with goat and human origin could be due to incorrect/ altered antigen presentation during T-cell priming resulting in a partial anergy of $C$. burnetii antigen-specific T cells [44]. As IFN- $\gamma$ cytokine produced by T-lymphocytes controls microbial infection [42], low IFN- $\gamma$ responses of peritoneal cells of mice infected with goat and human origin strains at 28 day p.i., could be favorable for $C$. burnetii survival and/or replication. This low inflammatory response could result in reduced protection and prevent/delay elimination of goat and human strains from the murine host leading to a persistent infection. In contrast, higher cell-mediated immune response induced by the cattle strains at later infection periods could provide improved protection by effectively inhibiting reinfections and promoting bacterial elimination. For further studies, phenotyping cells from peritoneal cavity of mice infected with various strains, will provide information on changes in the composition of immune cell populations in relation to IFN- $\gamma$ response kinetics, presence of poly-functional T cells and other cell-immunity related cytokine profiles (such as IL-2, IL-17). This information will improve insights into the immune responses that contribute to the protective immunity of various $C$. burnetii strain infections.

Persistently high cytokines and chemokines mRNA levels were induced in spleens of mice by cattle-derived strains throughout the infection period. However, no significant differences were 
observed between $C$. burnetii strains of different MLVA genotypes. IFN- $\gamma$ and inflammatory cytokines, such as TNF- $\alpha$, IL-1 $\beta$, IL-6, IL-12a secreted by antigen presenting cells (APC) as well as several inflammatory chemokines (CCL2, CCL6, CXCL10), were significantly higher in response to the cattle strains than to the goat and human strains, especially at later infection period. These high cytokine-chemokine levels as well as early stage IFN- $\gamma$ expression reflect a pro-inflammatory innate response of monocyte/ macrophages, NK and NKT cells. These initial splenic macrophage responses play a critical role in the expression of innate immune responses and likely drive and direct the ensuing adaptive immune response [45]. In addition, anti-inflammatory cytokines (IL-10, IL-1RA) were also higher in response to cattle strains than the other strains, showing a co-existence of both pro and anti-inflammatory responses. The anti-inflammatory responses are probably involved to balance the high pro-inflammatory responses generated by the immune system against antigens of cattle strains. Such a response could be critical to prevent uncontrolled inflammation and tissue damage as well as appropriate activation of inflammatory processes in the defense against the ongoing cattle strain infections $[46,47]$. IFN- $\gamma$ cytokine is suggested to be involved in the control of bacterial growth by enabling monocytes/macrophages to kill $C$. burnetii $[24,48,49]$. Other pro-inflammatory cytokines secreted by APC are known to play crucial roles in several aspects of the innate and adaptive immunity [42]. Among these, TNF- $\alpha$ is a key cytokine which has important functions in cell-mediated defense to intracellular bacteria. Along with IL-12, TNF- $\alpha$ is known to induce IFN- $\gamma$ production by NK cells, thereby enhancing the cytolytic activity of infected cells $[24,50]$. In mice infected with the cattle strains, TNF- $\alpha$, IL-12a and IFN- $\gamma$ cytokines were upregulated at 28 days p.i. and may play an essential role in controlling the infection. On the contrary, the TNF- $\alpha$ levels are very low in response to the goat and human strains at later infection period as compared to the control mice and mice infected with cattle strains. This may diminish the ability of the mice immune system to eliminate the infection caused by goat and human-derived strains (Fig 3a). Moreover, inflammatory chemokines were also at high levels in response to cattle strains and may be associated with directing immune cells to the site of infection resulting in a robust local immune response against invading pathogens [42]. As innate immunity can become active in the first minutes to hours after exposure to pathogens [51], a limitation of our study is that we did not measure cytokine responses at very early time points after the infection. Previous study on C. burnetii infection in a macrophage cell line has shown the induction of pro-inflammatory cytokines TNF- $\alpha$, IL-1 $\alpha$ within 3 hrs post-exposure [50]. Assessing very early cytokine responses in in vivo models will contribute to gain an improved understanding of the initial phase of the C. burnetii infection process.

Higher inflammatory cytokine and chemokine mRNA levels in response to cattle strains throughout the whole infection period, compared to the levels induced by goat and human strains, could induce a potent immune response. Such a response could result in the effective bacterial elimination as observed by decreased bacterial load in spleen as early as 21 day p.i. in response to cattle strains, as compared to goat and human strains (Supplementary Fig 1). Based on these 
observations, an early and effective bacterial clearance upon infection with cattle-derived strains can be speculated, which could lead to minimal or no clinical disease in natural hosts. Increased cytokine and chemokine mRNA levels only at early infection periods, as found for goat and human strains, could lead to an efficient control of the bacterial infection only during the early stages of the infection [24]. It can be speculated that the observed lower levels of cytokine and chemokines in response to goat and human strains at later infection periods, may facilitate persistent re-infections resulting in severe clinical disease outcomes. Another explanation of the observed high expression of inflammatory cytokines generated by cattle strains, could be due to the trained immunity (TI) phenomenon. TI has been previously reported for other bacterial species [52,53]. The cattle strain antigens could specifically induce epigenetic reprogramming in monocyte/macrophages resulting in TI. Such main innate immunity cells (like macrophages) can be trained leading to cell activation and enhanced pro-inflammatory cytokine production as host defense mechanisms [53-55]. The observed levels of cytokine production in response to the cattle strains (TNF- $\alpha$, IL-1 $\beta$, IL-6 and IL-12a) are majorly produced by macrophages. The persistent high levels of these cytokines could be due to the enhanced potential of macrophages upon bacterial re-infection through TI, leading to profound innate immune response against cattle strain antigens at later infection periods. TI is a well-documented phenomenon widely studied for the attenuated bacterial vaccine for tuberculosis [53]. In the same context, we could argue that cattle strain antigens, in contrast to goat and human strain antigens, might induce TI resulting in profound pro-inflammatory responses by host and as a result could qualify as potential vaccine candidates.

Differences in immune responses with respect to different host origins of C. burnetii strains, were mainly observed during the late phases of infections with cattle strains. This could implicate that high pro-inflammatory responses to cattle strains during late stages of the infection are critical for complete bacterial clearance and for inhibiting the development of chronic disease [56]. If true, than the chance of chronic disease development increases upon exposure to goat and human strains, because low immune responses were observed in mice in response to these strains which might aid in bacterial re-infections. Out of the four human strains used in the study, 3 of them originated from chronic Q fever patients. The data matches with the observation that persistent infections in humans result in chronic disease, while persistent infections of $C$. burnetii in goats has also been shown $[1,9,27,57]$. From our study we cannot prove whether indeed persistent infections of $C$. burnetii strains occurred in the mice, as our experiments lasted only for 28 days and a complete bacterial clearance from mice takes at least 8 weeks [18].

Apart from analyzing strains based on their host-origin, the strains were also differentiated based on their MLVA genotype classification. The strains of the CbNL01 genotype were predominant during the Dutch Q fever outbreak, whereas strains of the CbNL12 genotype were much less prevalent [6].The present study consistently showed a non-significant effect of genotype-grouped C. burnetii strains on the immune responses at all time points (Table 2). Comparative genomics of Dutch CbNL01 
and CbNL12 genotype strains, showed highly similar genomes of strains representing the same genotype (Kuley et al., unpublished). Although, the genome content differs between strains from different genotypes, we did not see major differences in immune responses. It could be possible that strains from the same host-origin but belonging to different genotypes share the same antigenic profile caused by specific point mutations in major antigens and/or by specific modifications of membrane proteins [58]. This could affect the bacterial recognition by the host immune system and the observed differential immune responses. For example; the cattle strains may encode surface antigens which are effectively recognized by the host immune system leading to more efficient defense against the pathogen. Besides, the goat and human strains may encode antigens which are not well recognized by the host resulting in defective activation or evasion of host immune system. Alternatively, it could also be possible that the bacteria undergo genomic re-organizations $[35,59,60]$ and/or alters its gene regulation in order to adapt to the host it resides in, resulting in host-origin specific but not genotypespecific immune responses. Based on our findings, the existence of shared factors in strains originating from the same host could be speculated in order to explain the observed host-origin dependent immune responses.

In the present study, temporal changes in immune responses were assessed in immunocompetent mice infected with $C$. burnetii strains. From our study we conclude that $C$. burnetii strains are able to induce both innate and adaptive immune responses, independent of the origin or the genotype of the strains. This indicates that both branches of the immune system are important for limiting C. burnetii infections [24,61]. The observation that cattle strains elicit higher immune response compared to goat and human strains, were also found in experiments using PBMCs of healthy humans stimulated with various inactivated $C$. burnetii strains from different host-origin (Ammerdorffer et al., Submitted). In these studies, higher levels of pro-inflammatory cytokines were observed in response to cattle strains as compared to others strains originating from goat, sheep and chronic human patients. Similar immune responses generated by strains originating from goat and human, may indicate similar virulence mechanisms in host. This could be due to the sharing of similar surface antigens or other adaptations in host which are not known yet. Based on our findings, we suggest that bacteria originating from goat hosts have a greater potential to cause outbreaks in humans and needs to be efficiently monitored. In this light, monitoring of cattle strains would be less important since these strains can generate stronger immune response in in vivo hosts and thus pose lower risk for Q fever outbreaks $[10,15,20]$.

\section{Acknowledgements}

We thank Ruth Bossers, Annemieke Dinkla and the animal care takers for their technical support. 


\section{References}

1. Maurin M, Raoult D. Q fever. Clin. Microbiol. Rev. 1999;12:518-53.

2. Angelakis E, Raoult D. Q fever. Vet. Microbiol. 2010;140:297-309.

3. Woldehiwet Z. Q fever (coxiellosis): epidemiology and pathogenesis. Res. Vet. Sci. 2004;77:93100 .

4. Palmer NC, Kierstead M, Key DW, Williams JC, Peacock MG, Vellend H. Placentitis and Abortion in Goats and Sheep in Ontario Caused by Coxiella burnetii. Can. Vet. J. 1983;24:60-1.

5. Arricau-Bouvery N, Rodolakis A. Is Q fever an emerging or re-emerging zoonosis? Vet. Res. 2005;36:327-49.

6. Roest HIJ, Ruuls RC, Tilburg JJHC, Nabuurs-Franssen MH, Klaassen CHW, Vellema P, et al. Molecular epidemiology of Coxiella burnetii from ruminants in Q fever outbreak, the Netherlands. Emerg. Infect. Dis. 2011;17:668-75.

7. Baca OG, Paretsky D. Q fever and Coxiella burnetii: a model for host-parasite interactions. Microbiol. Rev. 1983;47:127-49.

8. Honarmand H, Honarmand H. Q Fever: An Old but Still a Poorly Understood Disease, Q Fever: An Old but Still a Poorly Understood Disease. Interdiscip. Perspect. Infect. Dis. Interdiscip. Perspect. Infect. Dis. 2012;2012, 2012:e131932.

9. Mazokopakis EE, Karefilakis CM, Starakis IK. Q fever endocarditis. Infect. Disord. Drug Targets. 2010;10:27-31.

10. Raoult D, Marrie T, Mege J. Natural history and pathophysiology of Q fever. Lancet Infect. Dis. 2005;5:219-26.

11. Roest HIJ, Tilburg JJHC, van der Hoek W, Vellema P, van Zijderveld FG, Klaassen CHW, et al. The Q fever epidemic in The Netherlands: history, onset, response and reflection. Epidemiol. Infect. 2011;139:1-12.

12. Kampschreur LM, Hagenaars JCJP, Wielders CCH, Elsman P, Lestrade PJ, Koning OHJ, et al. Screening for Coxiella burnetii seroprevalence in chronic Q fever high-risk groups reveals the magnitude of the Dutch Q fever outbreak. Epidemiol. Infect. 2013;141:847-51.

13. Tilburg JJHC, Rossen JWA, van Hannen EJ, Melchers WJG, Hermans MHA, van de Bovenkamp $\mathrm{J}$, et al. Genotypic diversity of Coxiella burnetii in the 2007-2010 Q fever outbreak episodes in The Netherlands. J. Clin. Microbiol. 2012;50:1076-8.

14. Porten K, Rissland J, Tigges A, Broll S, Hopp W, Lunemann M, et al. A super-spreading ewe infects hundreds with Q fever at a farmers' market in Germany. BMC Infect. Dis. 2006;6:147.

15. Georgiev M, Afonso A, Neubauer H, Needham H, Thiery R, Rodolakis A, et al. Q fever in humans and farm animals in four European countries, 1982 to 2010. Euro Surveill. Bull. Eur. Sur Mal. Transm. Eur. Commun. Dis. Bull. 2013;18.

16. Muskens J, van Engelen E, van Maanen C, Bartels C, Lam TJGM. Prevalence of Coxiella burnetii infection in Dutch dairy herds based on testing bulk tank milk and individual samples by PCR and ELISA. Vet. Rec. 2011;168:79.

17. Tilburg JJHC, Roest HJIJ, Nabuurs-Franssen MH, Horrevorts AM, Klaassen CHW. Genotyping reveals the presence of a predominant genotype of Coxiella burnetii in consumer milk products. J. Clin. Microbiol. 2012;50:2156-8. 
18. Mori M, Boarbi S, Michel P, Bakinahe R, Rits K, Wattiau P, et al. In Vitro and In Vivo Infectious Potential of Coxiella burnetii: A Study on Belgian Livestock Isolates. PLoS ONE. 2013;8:e67622.

19. Roest HIJ, van Solt CB, Tilburg JJHC, Klaassen CHW, Hovius EK, Roest FTF, et al. Search for possible additional reservoirs for human Q fever, The Netherlands. Emerg. Infect. Dis. 2013;19:834-5.

20. Tilburg JJHC, Roest H-JIJ, Buffet S, Nabuurs-Franssen MH, Horrevorts AM, Raoult D, et al. Epidemic Genotype of Coxiella burnetii among Goats, Sheep, and Humans in the Netherlands. Emerg. Infect. Dis. 2012;18:887-9.

21. To H, Htwe KK, Kako N, Kim HJ, Yamaguchi T, Fukushi H, et al. Prevalence of Coxiella burnetii infection in dairy cattle with reproductive disorders. J. Vet. Med. Sci. 1998;60:859-61.

22. Roest HJ, van Steenbergen J, Wijkmans C, van Duijnhoven Y, Stenvers O, Oomen T, et al. [Q fever in 2008 in the Netherlands and the expectations of 2009]. Tijdschr. Diergeneeskd. 2009;134:300-3.

23. Roest HJ. Coxiella burnetii in pregnant goats. 2013.

24. Andoh M, Zhang G, Russell-Lodrigue KE, Shive HR, Weeks BR, Samuel JE. T Cells Are Essential for Bacterial Clearance, and Gamma Interferon, Tumor Necrosis Factor Alpha, and B Cells Are Crucial for Disease Development in Coxiella burnetii Infection in Mice. Infect. Immun. 2007;75:3245-55.

25. Shannon JG, Heinzen RA. Adaptive immunity to the obligate intracellular pathogen Coxiella burnetii. Immunol. Res. 2009;43:138-48.

26. Kuley R, Smith HE, Janse I, Harders FL, Baas F, Schijlen E, et al. First Complete Genome Sequence of the Dutch Veterinary Coxiella burnetii Strain NL3262, Originating from the Largest Global Q Fever Outbreak, and Draft Genome Sequence of Its Epidemiologically Linked Chronic Human Isolate NLhu3345937. Genome Announc. [Internet]. 2016 [cited 2016 Jun 28];4. Available from: http://www.ncbi.nlm.nih.gov/pmc/articles/PMC4841129/

27. Roest H-J, van Gelderen B, Dinkla A, Frangoulidis D, van Zijderveld F, Rebel J, et al. Q Fever in Pregnant Goats: Pathogenesis and Excretion of Coxiella burnetii. PLoS ONE. 2012;7:e48949.

28. Kuley R, Smith HE, Frangoulidis D, Smits MA, Jan Roest HI, Bossers A. Cell-Free Propagation of Coxiella burnetii Does Not Affect Its Relative Virulence. PLoS ONE [Internet]. 2015 [cited 2015 Apr 12];10. Available from: http://www.ncbi.nlm.nih.gov/pmc/articles/PMC4368683/

29. Lukácová M, Kazár J, Gajdosová E, Vavreková M. Phase variation of lipopolysaccharide of Coxiella burnetii, strain Priscilla during chick embryo yolk sac passaging. FEMS Microbiol. Lett. 1993;113:285-9.

30. Schramek S, Galanos C. Lipid A component of lipopolysaccharides from Coxiella burnetii. Acta Virol. 1981;25:230-4.

31. Hitchcock PJ, Brown TM. Morphological heterogeneity among Salmonella lipopolysaccharide chemotypes in silver-stained polyacrylamide gels. J. Bacteriol. 1983;154:269-77.

32. Mahlknecht P, Stemberger S, Sprenger F, Rainer J, Hametner E, Kirchmair R, et al. An antibody microarray analysis of serum cytokines in neurodegenerative Parkinsonian syndromes. Proteome Sci. 2012;10:71.

33. Kuley R, Bossers-deVries R, Smith HE, Smits MA, Roest HIJ, Bossers A. Major differential gene regulation in Coxiella burnetii between in vivo and in vitro cultivation models. BMC Genomics. 2015;16:953. 
34. de Jonge HJM, Fehrmann RSN, de Bont ESJM, Hofstra RMW, Gerbens F, Kamps WA, et al. Evidence Based Selection of Housekeeping Genes. PLoS ONE [Internet]. 2007 [cited 2016 Sep 23];2. Available from: http://www.ncbi.nlm.nih.gov/pmc/articles/PMC1976390/

35. Beare PA, Samuel JE, Howe D, Virtaneva K, Porcella SF, Heinzen RA. Genetic Diversity of the Q Fever Agent, Coxiella burnetii, Assessed by Microarray-Based Whole-Genome Comparisons. J. Bacteriol. 2006;188:2309-24.

36. Russell-Lodrigue KE, Andoh M, Poels MWJ, Shive HR, Weeks BR, Zhang GQ, et al. Coxiella burnetii isolates cause genogroup-specific virulence in mouse and guinea pig models of acute $\mathrm{Q}$ fever. Infect. Immun. 2009;77:5640-50.

37. Glazunova O, Roux V, Freylikman O, Sekeyova Z, Fournous G, Tyczka J, et al. Coxiella burnetii Genotyping. Emerg. Infect. Dis. 2005;11:1211-7.

38. Svraka S, Toman R, Skultety L, Slaba K, Homan WL. Establishment of a genotyping scheme for Coxiella burnetii. FEMS Microbiol. Lett. 2006;254:268-74.

39. Roest HIJ, Post J, van Gelderen B, van Zijderveld FG, Rebel JMJ. Q fever in pregnant goats: humoral and cellular immune responses. Vet. Res. 2013;44:67.

40. Dupuis G, Péter O, Peacock M, Burgdorfer W, Haller E. Immunoglobulin responses in acute Q fever. J. Clin. Microbiol. 1985;22:484-7.

41. Teixeira LK, Fonseca BPF, Barboza BA, Viola JPB. The role of interferon-gamma on immune and allergic responses. Mem. Inst. Oswaldo Cruz. 2005;100 Suppl 1:137-44.

42. Commins SP, Borish L, Steinke JW. Immunologic messenger molecules: cytokines, interferons, and chemokines. J. Allergy Clin. Immunol. 2010;125:S53-72.

43. Skeen MJ, Ziegler HK. Activation of gamma delta $T$ cells for production of IFN-gamma is mediated by bacteria via macrophage-derived cytokines IL-1 and IL-12. J. Immunol. Baltim. Md 1950. 1995;154:5832-41.

44. Böcher WO, Galun E, Marcus H, Daudi N, Terkieltaub D, Shouval D, et al. Reduced hepatitis B virus surface antigen-specific Th1 helper cell frequency of chronic HBV carriers is associated with a failure to produce antigen-specific antibodies in the trimera mouse. Hepatol. Baltim. Md. 2000;31:480-7.

45. Dorhoi A, Kaufmann SHE. Fine-tuning of T cell responses during infection. Curr. Opin. Immunol. 2009;21:367-77.

46. Tamayo E, Fernández A, Almansa R, Carrasco E, Heredia M, Lajo C, et al. Pro- and antiinflammatory responses are regulated simultaneously from the first moments of septic shock. Eur. Cytokine Netw. 2011;22:82-7.

47. Ward NS, Casserly B, Ayala A. The Compensatory Anti-inflammatory Response syndrome (CARS) in Critically ill patients. Clin. Chest Med. 2008;29:617-viii.

48. Ghigo E, Honstettre A, Capo C, Gorvel J-P, Raoult D, Mege J-L. Link between impaired maturation of phagosomes and defective Coxiella burnetii killing in patients with chronic Q fever. J. Infect. Dis. 2004;190:1767-72.

49. Ghigo E, Capo C, Tung C-H, Raoult D, Gorvel J-P, Mege J-L. Coxiella burnetii Survival in THP-1 Monocytes Involves the Impairment of Phagosome Maturation: IFN- $\gamma$ Mediates its Restoration and Bacterial Killing. J. Immunol. 2002;169:4488-95. 
50. Tujulin E, Lilliehöök B, Macellaro A, Sjöstedt A, Norlander L. Early cytokine induction in mouse P388D1 macrophages infected by Coxiella burnetii. Vet. Immunol. Immunopathol. 1999;68:159-68.

51. Hoffmann JA, Kafatos FC, Janeway CA, Ezekowitz RA. Phylogenetic perspectives in innate immunity. Science. 1999;284:1313-8.

52. Mehta S, Jeffrey KL. Beyond receptors and signaling: epigenetic factors in the regulation of innate immunity. Immunol. Cell Biol. 2015;93:233-44.

53. van der Meer JWM, Joosten LAB, Riksen N, Netea MG. Trained immunity: A smart way to enhance innate immune defence. Mol. Immunol. 2015;68:40-4.

54. Netea MG, Quintin J, van der Meer JWM. Trained immunity: a memory for innate host defense. Cell Host Microbe. 2011;9:355-61.

55. Netea MG, Joosten LAB, Latz E, Mills KHG, Natoli G, Stunnenberg HG, et al. Trained immunity: A program of innate immune memory in health and disease. Science. 2016;352:aaf1098.

56. Manderscheid PA, Bodkin RP, Davidson BA, Jensen E, Russo TA, Knight PR. Bacterial Clearance and Cytokine Profiles in a Murine Model of Postsurgical Nosocomial Pneumonia. Clin. Diagn. Lab. Immunol. 2004;11:742-51.

57. Berri M, Rousset E, Champion JL, Russo P, Rodolakis A. Goats may experience reproductive failures and shed Coxiella burnetii at two successive parturitions after a Q fever infection. Res. Vet. Sci. 2007;83:47-52.

58. D'Amato F, Rouli L, Edouard S, Tyczka J, Million M, Robert C, et al. The genome of Coxiella burnetii Z3055, a clone linked to the Netherlands Q fever outbreaks, provides evidence for the role of drift in the emergence of epidemic clones. Comp. Immunol. Microbiol. Infect. Dis. 2014;37:281-8.

59. Beare PA, Unsworth N, Andoh M, Voth DE, Omsland A, Gilk SD, et al. Comparative genomics reveal extensive transposon-mediated genomic plasticity and diversity among potential effector proteins within the genus Coxiella. Infect. Immun. 2009;77:642-56.

60. Pallen MJ, Wren BW. Bacterial pathogenomics. Nature. 2007;449:835-42.

61. Kampschreur LM, Oosterheert JJ, Koop AMC, Wegdam-Blans MCA, Delsing CE, BleekerRovers CP, et al. Microbiological challenges in the diagnosis of chronic Q fever. Clin. Vaccine Immunol. CVI. 2012;19:787-90. 


\section{Supplementary figure}

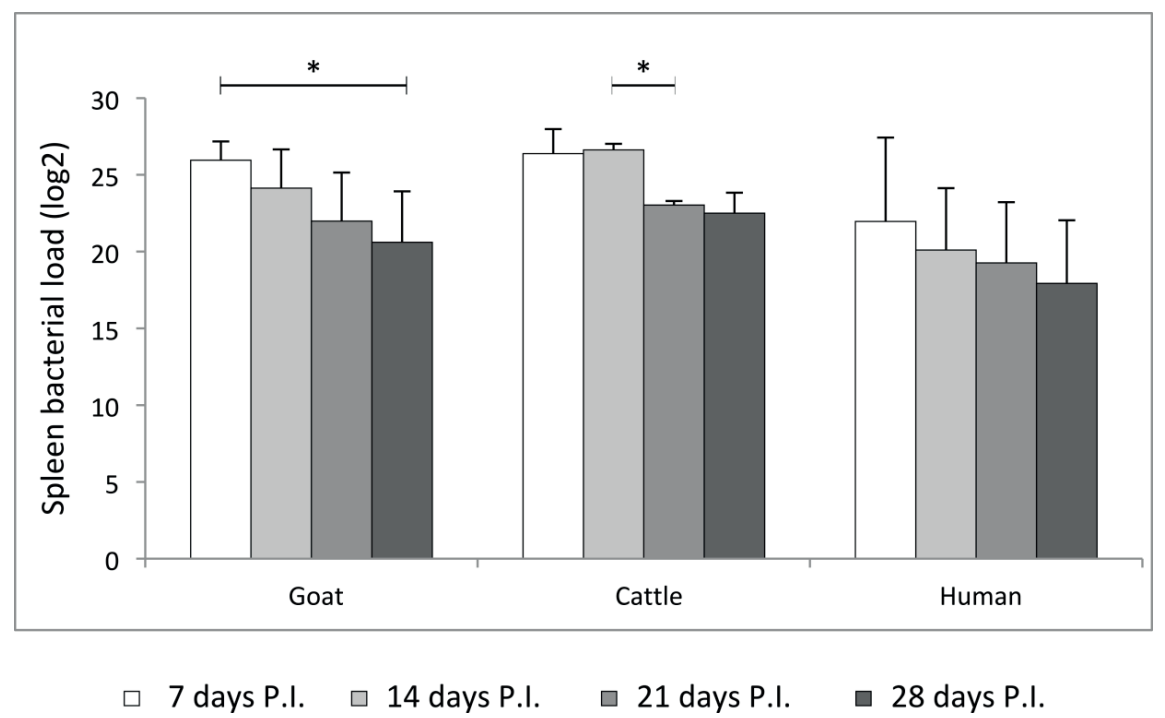

Figure S1. Splenic bacterial loads in mice infected with goat, cattle and human strains at 7, 14, 21 and 28 day time points. Significant decrease in bacterial load was observed comparatively early at 21 day p.i. in cattle infected group. Significant differences within infected groups were indicated as $*(p<0.05)$. 



\section{Chapter 7}

\section{Coxiella burnetii isolates originating}

from infected cattle induce a more pronounced pro-inflammatory cytokine response compared to

\section{isolates from infected goats and sheep}

Anne Ammerdorffer, Runa Kuley, Annemieke Dinkla, Leo A.B. Joosten, Rudolf Toman, Hendrik-Jan Roest, Tom Sprong, Johanna M. Rebel

Pathogens and Disease, accepted for publication in an adapted format 


\begin{abstract}
Coxiella burnetii is the causative agent of $\mathrm{Q}$ fever. Although the prevalence of $C$. burnetii in cattle is much higher than in goats and sheep, infected cattle are rarely associated with human outbreaks. We investigated whether the immune response of humans differs after contact with $C$. burnetii isolates from different host origins or with different multi-locus variable number of tandem repeat analysis (MLVA) genotypes.

Cytokine responses were measured in human peripheral blood mononuclear cells (PBMCs) stimulated with 16 C. burnetii isolates with known MLVA genotype from goats, sheep, cattle, acute and chronic $\mathrm{Q}$ fever patients. C. burnetii isolates originating from cattle induce significantly more IL$1 \beta$, TNF- $\alpha$ and IL-22 than the isolates from goats, sheep or chronic Q fever patients. Comparing the cytokine induction of the isolates based on their MVLA genotype did not reveal differences in response between the MLVA genotypes. The pro-inflammatory cytokine response induced in human PBMCs by C. burnetii isolates from cattle may explain the low incidence of human $\mathrm{Q}$ fever outbreaks caused by cattle. The cytokine profile of PBMCs stimulated with C. burnetii isolates from chronic Q fever patients resembles isolates from goats. Furthermore, cytokine responses seem to be depending on host origin than on MLVA genotype.
\end{abstract}




\section{Introduction}

The zoonotic bacterium Coxiella burnetii caused a large outbreak in the Netherlands between 2007 and 2010. More than 4.000 acute Q fever cases were reported, but seroprevalence studies estimated at least 40.000 individuals infected with C. burnetii $[1,2]$. Besides acute $\mathrm{Q}$ fever, C. burnetii infection can lead to chronic Q fever in $1-5 \%$ of the individuals after an initial infection [3]. The main sources for $\mathrm{Q}$ fever in humans are sheep and goats. In contrast to the observed clinical symptoms in humans during $C$. burnetii infection, infection in ruminants is mostly asymptomatic. However, in pregnant sheep and goats infection may cause abortion, premature birth, dead or weak offspring and it may cause reproductive disorders in cattle [4, 5]. In a large review study, overarching the years 1982 to 2010, was conducted in which, among others, the seroprevalence of C. burnetii-antibodies in goats, sheep and cattle was investigated [6]:within-herd prevalence estimates for cattle, goat and sheep were respectively 20.8\%, 40.0\%, 56.9\% (Bulgaria), 15.0\%, 88.1\%, 20.0\% (France), 19.3\%, 2.5\%, 8.7\% (Germany), and $21.0 \%, 7.8 \%, 3.5 \%$ (the Netherlands); the herd prevalence (a herd is considered positive when at least one animal in the herd was serologically confirmed) in the Netherlands was up to $37.0 \%$ in cattle, $17.8 \%$ in goats and $14.5 \%$ in sheep. In France, the herd prevalence for cattle, goats and sheep was respectively $73.0 \%, 40 \%$ and $89 \%$ [6]. In 2011 and specifically for cattle in the Netherlands, Muskens et al [7] identified that 268 of 341 cattle herds (78.6\%) tested positive for $C$. burnetii antibodies and at $56.6 \%$ of the herds C. burnetii DNA was detected.

Despite the high percentage of (herd) seroprevalence of C. burnetii in cattle, only a minority of the human Q fever outbreaks are attributed to cattle [6]. In addition, while almost all commercial milk products from cattle contain C. burnetii DNA, to our knowledge no reports are available indicating that these farmers, their family or milk consumers developed Q fever [8]. Several studies indicate that specific genotypes of $C$. burnetii are associated with cattle [8-10]. However, cattle can also be infected with other genotypes, when more animal species are present on the same farm [11]. It can be hypothesized that humans can become infected by $C$. burnetii isolates originating from both cattle and goat, but infection with cattle isolates might result in fewer, milder or no clinical symptoms compared with infections by goat isolates. We hypothesize that the cattle $C$. burnetii isolates induce a more profound immune response in humans, which can eventually result in more effective elimination of the bacteria leading to absence of clinical disease.

To our knowledge, the current literature does not comprise studies focusing on the human immune response against different $C$. burnetii isolates. In order to check our hypothesis, we conducted the present study in which we used 16 C. burnetii isolates with either a different origin according to the host species and/or different MLVA genotype to investigate their cytokine induction upon stimulation of human peripheral blood mononuclear cells (PBMCs). As humans can get infected via either goat, sheep or cattle, C. burnetii isolates retrieved from these animals were included in the study. In addition, isolates from both acute and chronic Q fever patients were included to examine whether they induced a comparable cytokine pattern as the animal isolates. Our findings reveal that the 
origin of the C. burnetii isolates has a greater influence on the cytokine production by human PBMCs, compared to the MLVA genotype. Here we provide a possible immunological explanation why $C$. burnetii transmission from infected cattle are less likely to induce infection in humans, which can relate to the minimal numbers of human $\mathrm{Q}$ fever outbreaks caused by cattle as stated in the current literature.

\section{Material and methods}

\section{C. burnetii isolates}

The C. burnetii isolates X09003262-001 (3262), CbCVIC1, 8014160-001 (14160-001), 8014160-002 (14160-002) and 18430 were isolated from the placenta of goats or sheep that aborted at different farms during the $\mathrm{Q}$ fever outbreak in the Netherlands. The human C. burnetii isolates from the Netherlands, 42785537 and 334937 were isolated from the heart valve from two individual chronic Q fever patients from the Radboud University Medical Centre and Canisius Wilhelmina Hospital respectively. The other human isolates, Schperling, Henzerling, Herzberg and Scurry were a kind gift from Dimitrios Frangoulidis of the Bundeswehr Institute for Microbiology (Munich, Germany). These isolates were retrieved from acute $\mathrm{Q}$ fever patients, however they were not obtained in the Netherlands during the outbreak, and background information about the assumed origin (infection via goat, sheep or cattle) is missing. Four isolates were obtained in France from goats (1683CbC2 and CbG15D6), sheep (736Cb01) and cattle (701CbB1) and were kindly provided by A. Rodolakis (INRA, France). The second cattle isolate (CbBEB1) came from Belgium and was a kind gift from D. Fretin (Coda Cerva, Bruxelles). All isolates represent LPS phase I. LPS phase determination was performed by SDS-PAGE and silver staining, using purified phase I (RSA493) and phase II (RSA439) C. burnetii NM LPS (kindly provided by R. Toman) as controls [12, 13]. More details of the isolates, like origin of the host (referred throughout the article as 'origin'), country and MLVA genotype is described in Table 1. All isolates were cultured on Buffalo Green Monkey (BGM) cells as described previously and heat inactivated at $99{ }^{\circ} \mathrm{C}$ for $30 \mathrm{~min}$ [14]. All isolated were quantified by qPCR as described in detail by Roest et al. [15]. In short, the qPCR targets a single copy gene encoding a C. burnetii-specific hypothetical protein (gene bank number AY502846) using the forward primer 5'ATAGCGCCAATCGAAATGGT-3', the reverse primer 5'- CTTGAATACCCATCCCGAAGTC-3', and the NED-labelled probe 5'-CCCAGTAGGGCAGAAGACGTTCCCC-3'. qPCR was performed on a 7500 Fast Real Time PCR system (Applied Biosystems, USA),

\section{MLVA genotyping}

MLVA genotyping was performed by using a selection of 12 loci: ms01, ms03, ms20, ms21, ms22, $\mathrm{ms} 24, \mathrm{~ms} 27, \mathrm{~ms} 28, \mathrm{~ms} 30, \mathrm{~ms} 31, \mathrm{~ms} 24$ and ms36. The method of MLVA genotyping is described in detail by Roest et al. and Arricau-Bouvery et al. [10, 16]. A dendrogram based on the MLVA genotype of the isolates was made using BioNumerics 7.5. 


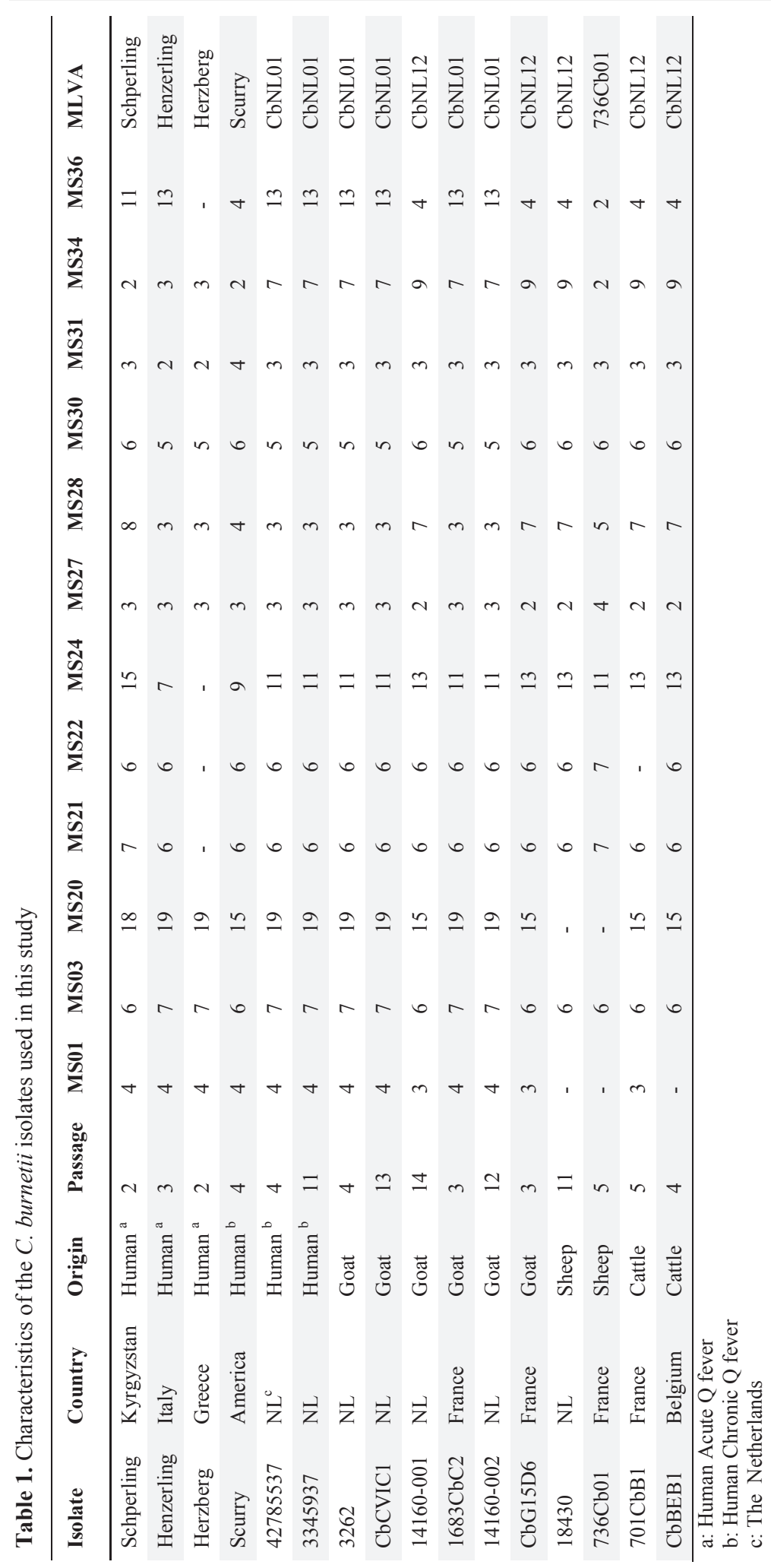


Isolation of human peripheral blood mononuclear cells and stimulation with $C$. burnetii PBMCs from healthy individuals were isolated according to standard protocols (Invitrogen, USA) as described previously [17]. $5 \times 10^{5}$ cells were added to a round-bottom 96-well plate (Corning, The Netherlands) and incubated with RPMI medium and the 16 C. burnetii isolates $\left(1 \times 10^{\wedge} 7 / \mathrm{ml}\right)$ in the presence of $10 \%$ human serum. After 24 hours, 48 hours and 7 days supernatants were harvested, depending on the cytokine to be measured, and stored at $-20^{\circ} \mathrm{C}$.

\section{Cytokine measurements}

Cytokine production was measured in the cell supernatants using ELISA according to the manufacturer's protocols. The following kits were used: IL-10 and IFN- $\gamma$ (Sanquin, the Netherlands), TNF- $\alpha$, IL-1 $\beta$, IL-1Ra, IL-17 and IL-22 (R\&D Systems, USA). Absorption was measured at $450 \mathrm{~nm}$ using a microplate reader (Bio-Rad Laboratories, the Netherlands). TNF- $\alpha$ and IL-1 $\beta$ were measured after $24 \mathrm{~h}$, IL-10 and IL-1Ra after $48 \mathrm{~h}$ and IFN- $\gamma$, IL-17 and IL-22 were measured after $7 \mathrm{~d}$ incubation.

\section{Statistics}

PBMCs of 11 healthy individuals were stimulated with the different $C$. burnetii isolates. The average cytokine production for each isolate was used in a one-way Anova to identify the differences between the groups. In case of IL-1 $\beta$, TNF- $\alpha$, IL-10, IL-1Ra, IL-17 and IL-22 the homogeneity of variances was $\geq 0.05$, therefore the Bonferonni Post-Hoc test was used to compare the different groups. For IFN$\gamma$, difference between the variances in the groups $(<0.05)$ was calculated and the Dunnett T3 Post-Hoc test was used. The data are expressed as mean ( \pm standard error of the mean). IBM SPSS 18 software was used to perform the statistical analyses and GraphPad Prism 5 software was used generate the graphs. Differences with a $\mathrm{P}$ value of $<0.05$ were considered statistically significant.

\section{Results}

\section{C. burnetii isolates from cattle induce a pro-inflammatory cytokine response}

PBMCs stimulated with cattle isolates produced significantly more of the pro-inflammatory cytokines IL-1 $\beta$ and TNF- $\alpha$, compared to the sheep, goat or isolates from patients with chronic Q fever (Figure 1A). In contrast, no significant differences were found between the cattle and the isolates from acute human cases. In case of IFN- $\gamma$, human PBMCs produced similar IFN- $\gamma$ levels after stimulation with all different $C$. burnetii isolates. The general induction of IFN- $\gamma$ was much lower than for IL- $1 \beta$ and TNF$\alpha$. The fold induction of IL-1 $\beta$ ranged from 10x in sheep and more than $40 \mathrm{x}$ in cattle isolates compared with no stimulation, whereas, the fold induction for IFN- $\gamma$ was only $1.44 \mathrm{x}$ and $2.6 \mathrm{x}$ in sheep and cattle isolates respectively. Besides the high pro-inflammatory cytokine induction of cattle isolates, the isolates from acute human cases also produced high amounts of IL-1 $\beta$ and TNF- $\alpha$. In case of IL-1 $\beta$ 

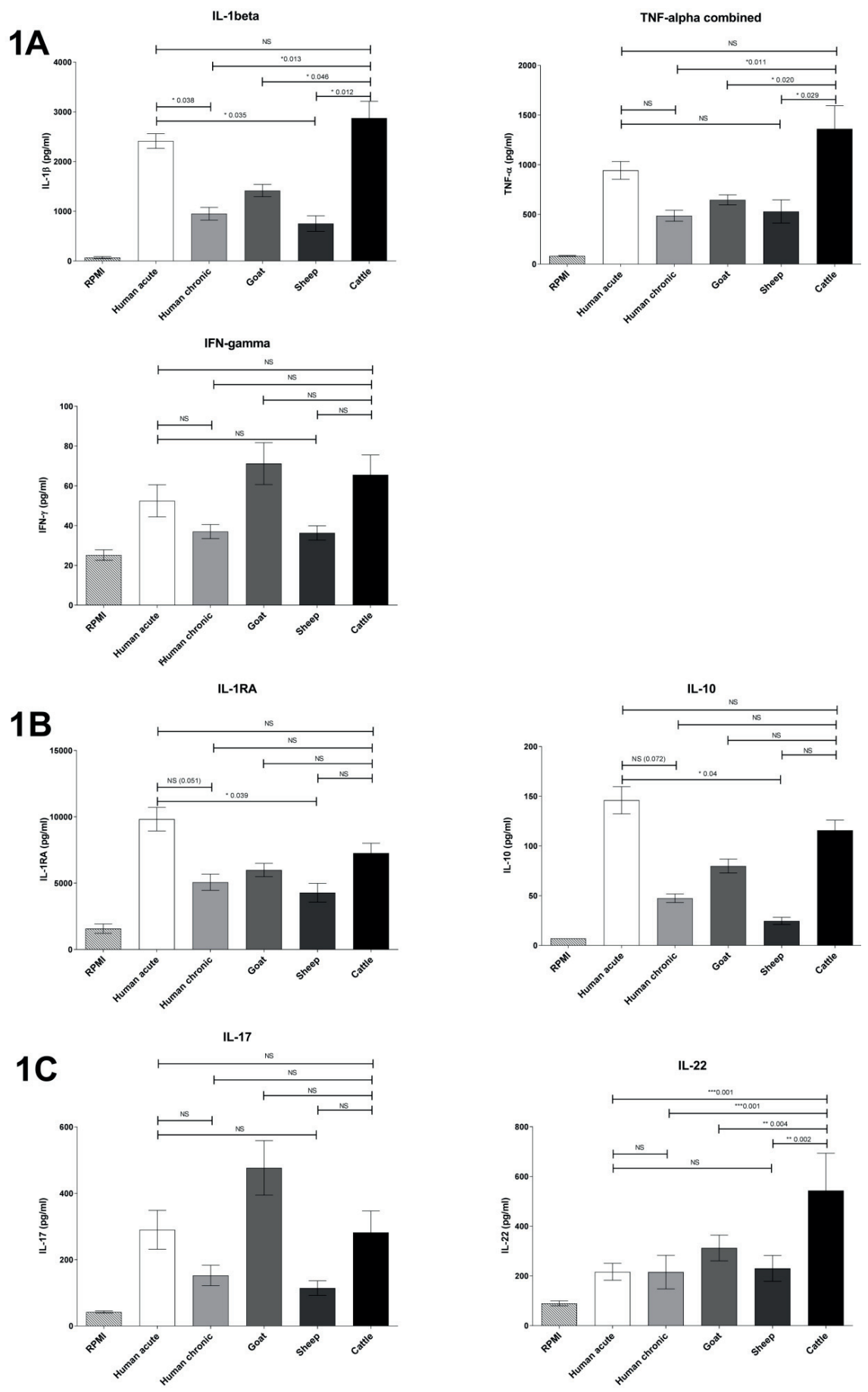
Figure 1. Cytokine profiles of the C. burnetii isolates grouped on their origin. PBMCs of 11 healthy individuals were stimulated with RPMI medium (striped bar) and 16 different $C$. burnetii isolates, see Table 1 for the details of the isolates. The following cytokines were measured using ELISA: IL-1 $\beta$, TNF- $\alpha$, IFN- $\gamma$ (1A), IL-Ra, IL-10 (1B), IL-17 and IL-22 (1C). The color of the bars match the following isolates; white (3x chronic human), light grey ( $3 \mathrm{x}$ acute human), medium grey (6x goat), dark grey (2x sheep), black (2x cattle). Statistical differences were tested using one-way ANOVA. In case of all cytokines, except IFN- $\gamma$, the Bonferonni Post-Hoc test was used. For IFN- $\gamma$, differences were calculates using Fishers Least Significant Difference (LSD). NS: Not Significant, ${ }^{*} \mathrm{p}<0.05, * * \mathrm{p}<0.01, * * * \mathrm{p}<0.001$. Data is presented as mean \pm SEM.

there was a significant difference between isolates from patients with acute Q fever compared to isolates derived from chronic $\mathrm{Q}$ fever patients $(\mathrm{p}=0.038)$ and sheep isolates $(\mathrm{p}=0.035)$ (Figure $1 \mathrm{~A})$. In addition to the pro-inflammatory cytokines, the anti-inflammatory cytokines IL-1Ra and IL-10 were measured. Isolates from acute $\mathrm{Q}$ fever patients induced the highest IL-10 and IL-1Ra in human PBMCs, followed by the cattle isolates (no significant differences). Comparing the isolates from chronic and acute $\mathrm{Q}$ fever patients show a trend for a lower IL-10 ( $\mathrm{p}=0.072)$ and IL-1Ra ( $\mathrm{p}=0.051)$ in the isolates from chronic Q fever patients (Figure 1B). Finally the Th17 cytokines, IL-17 and IL-22 were measured. For IL-17, no differences were observed between the groups. In contrast, the IL-22 response was significantly higher in human PBMCs stimulated with cattle isolates compared to the other groups, the differences are significant for sheep $(\mathrm{p}=0.002)$ goat $(\mathrm{p}=0.004)$ and isolates from acute and chronic $\mathrm{Q}$ fever patients (both $\mathrm{p}=0.001$ ) (Figure 1C). Comparison of the isolates derived from infected cattle and the isolates from individuals with acute $\mathrm{Q}$ fever show that except for IL-22, there are no significant differences in cytokine responses in human PBMCs. Three of the six goat isolates have a low passage number (3-4 times), while the other three isolates have been passed on BGM cells 12 to 14 times. Despite these differences in passage number, examination by SDS-PAGE and silver staining revealed that all isolates contained phase I LPS. Furthermore, statistical analyses between the low and high passage number goat isolates showed no significant difference in IL-1 $\beta$ and TNF- $\alpha$ production by human PBMCs upon stimulation (data not shown).

\section{The MLVA genotype of $C$. burnetii isolates does not influence the cytokine production of human PBMCs}

Besides analyzing the isolates based on their origin, the isolates were discriminated based on their MLVA genotype. Two main genotypes can be distinguished among our isolates. First the genotype, named CbNL01 by Roest et al. [10], was the dominant genotype found during the Dutch Q fever outbreak. In total six isolates from our study belong to this genotype, four goats and two isolates from 


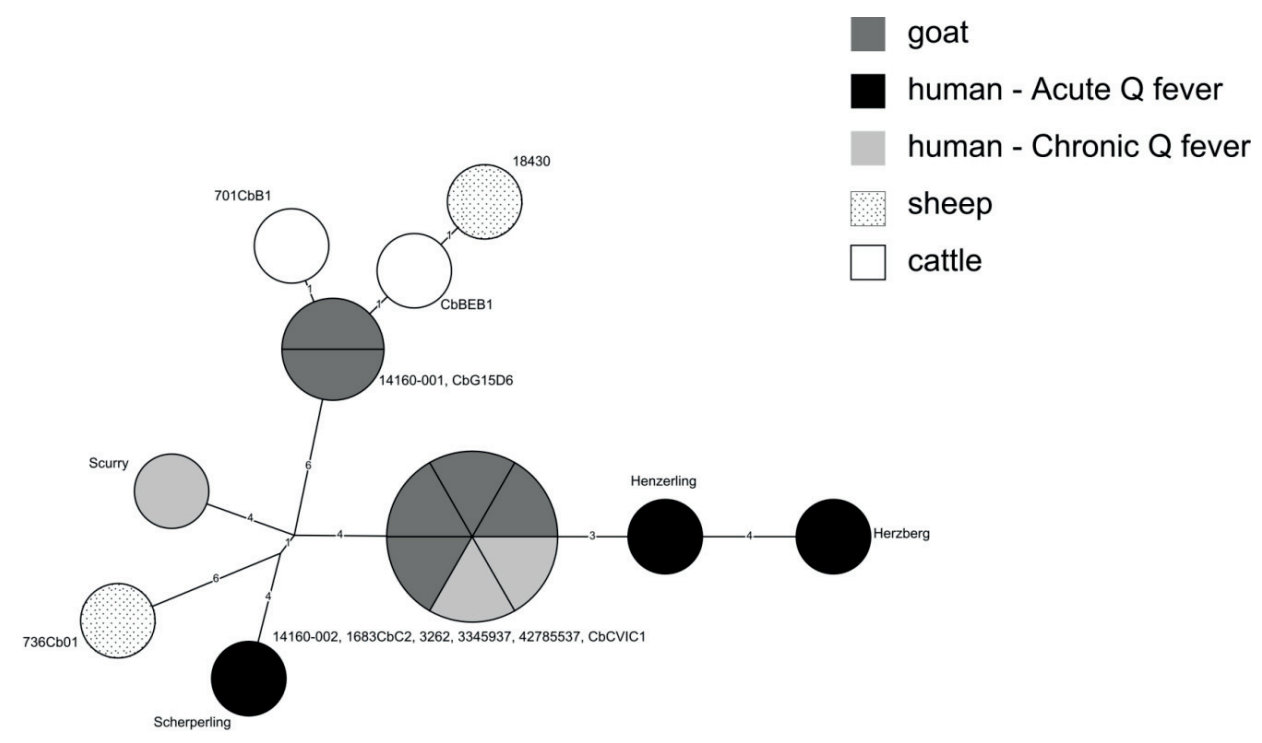

Figure 2. Minimum spanning tree analysis of MLVA genotypes from C. burnetii isolates used in the study. A total of 12 micro satellite loci (Table 1) were used to construct a dendrogram of 16 C. burnetii isolates. Numbers on the connecting lines refer to the distance between the nodes, e.g. 4 means four different loci. The size of the circles is proportional to the number of strains bearing the same genetic profile. Colors indicate the origin from which the isolates originate; goat (dark grey), human acute $\mathrm{Q}$ fever (black), human chronic Q fever (light grey), sheep (dashed) and cattle (white). Branch labels give the. Clustering by minimum spanning tree (MST, hypothetical, non-rooted) was performed with Bionumerics 7.5.

chronic Q fever patients. The second main genotype in our study, referred to as CbNL12, contained two goat, one sheep and the two cattle isolates. The third group consists of the remaining individual genotypes, including the three acute human strains, one chronic human strain and a second sheep strain. The differences in MLVA genotype are visualized in Figure 2, which shows the minimal spanning tree of all C. burnetii isolates included in this study. Genotype CbNL12 differs with 9 micro satellite (ms) loci from CbNL01 and is situated in a separate genotype. From three isolates, 701CbB1, CbBEB1 and 18430, one or two ms loci were missing. Based on the 10 known ms loci, these isolates belong to the CbNL12 MLVA genotype.

Comparison of the cytokine induction between the diverse genotypes showed no significant differences in the pro-inflammatory cytokines (IL-1 $\beta, \mathrm{TNF}-\alpha$, IFN- $\gamma$ ) (Figure 3A) and other cytokines (IL-10, IL-1Ra, IL-17 and IL-22) (Figure 3B and 3C). Among the isolates with the CbNL12 MLVA genotype, the isolates originated from infected cattle produced significant more TNF- $\alpha$, IL-1 $\beta$, IL-10 and IL-1Ra (Supplement figure 1). 
3A

IL-1 $\beta$

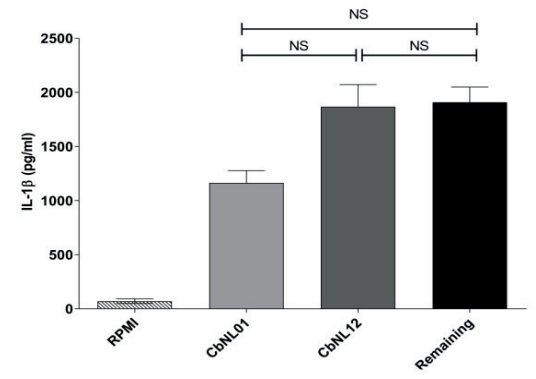

IFN- $\gamma$

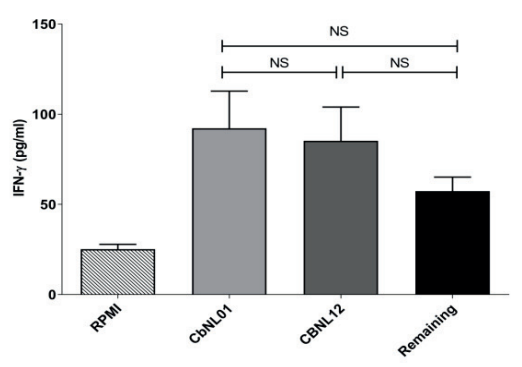

3B

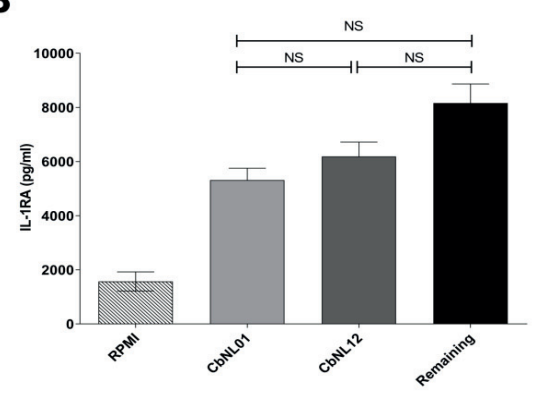

$3 C$

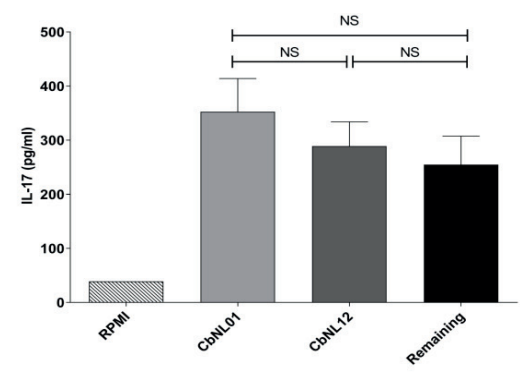

TNF-alpha

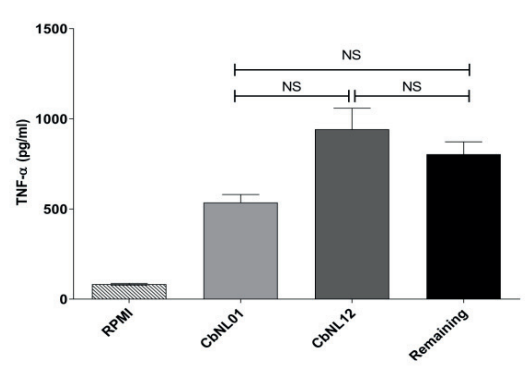

IL-10

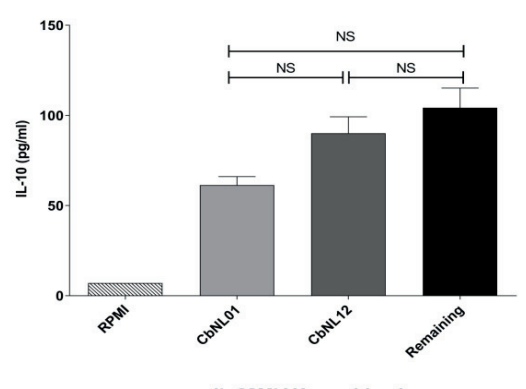

IL-22MLVA combined

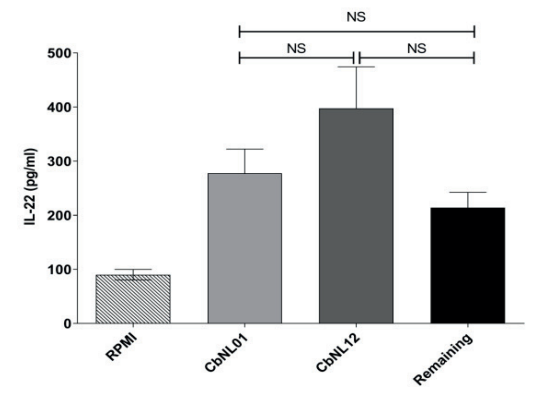


Figure 3. Cytokine profiles of the C. burnetii isolates grouped on their MLVA genotype. PBMCs of 11 healthy individuals were stimulated with RPMI medium (striped bar) and 16 different C. burnetii isolates, see Table 1 for the details of the isolates. The following cytokines were measured using ELISA: IL-1 $\beta$, TNF- $\alpha$, IFN- $\gamma(\mathbf{3 A})$, IL-Ra, IL-10 (3B), IL-17 and IL-22 (3C). The color of the bars match the following isolates; white (6x CbNL01), grey (5x CbNL12), black (5x remaining MLVA genotypes). Statistical differences were tested using one-way ANOVA. In case of all cytokines, except IFN- $\gamma$, the Bonferonni Post-Hoc test was used. For IFN- $\gamma$, differences were calculates using Fishers Least Significant Difference (LSD). NS: Not Significant. Data is presented as mean \pm SEM.

\section{Discussion}

C. burnetii causes Q fever in humans and a broad range of animals throughout the world. In the majority of the examined cases, goats and sheep are the most likely source for human Q fever outbreaks [6]. Interestingly, in the Netherlands and Germany, the herd prevalence of C. burnetii in cattle is much higher than in goat and sheep. This could suggest that infected cattle are a large threat for the human population [6]. However, in the Netherlands and other countries with known human Q fever outbreaks, cattle are scarcely the source of the outbreak [6]. We hypothesized that these cattle $C$. burnetii isolates induce a more profound immune response in humans, which can eventually result in more effective elimination of the bacteria, resulting in less clinical disease.

Our study revealed that the cytokine patterns induced in human PBMCs depends on the $C$. burnetii isolate host origin. Namely, the two cattle isolates induced higher amounts of IL-1 $\beta$, TNF- $\alpha$, and IL-22 in human PBMCs than the goat and sheep isolates. In addition, we observed that the MLVA genotype of the $C$. burnetii isolates is not related to the cytokine induction by PBMCs. As the cytokine patterns induced in human PBMCs by C. burnetii isolates (grouped on base of their MLVA genotype), revealed no significant differences between the groups.

There are several explanations for the observed differences in cytokine response of human PBMCs against $C$. burnetii isolates originating from different animal hosts, and for the observation that the MLVA genotypes used in this study do not effect these cytokine responses. We observed that the two cattle isolates with MLVA genotype CbNL12 induced more pro- and antiinflammatory cytokines in human PBMCs than the two goats and sheep isolates with the same MLVA genotype (Supplement figure 1). However, it is not possible to correlate the cytokine induction by human PBMCs against a C. burnetii isolate based on its MLVA genotype. MLVA genotyping depends on a selection of loci containing tandem repeats are targeted. These loci do probably not code for antigens that are involved in the innate immune response. So, even though the MLVA genotype of the C. burnetii isolates is the same, other parts of the genome can differ which might result in variations in antigens responsible for diverse responses from host [18]. 
Another explanation for the observed differences in cytokine response upon stimulation by $C$. burnetii isolates coming from different hosts is the expression of diverse virulence factors. Recently, D'Amato et al. indicated that C. burnetii Z3055, a clone linked to the Dutch Q fever outbreak isolate 3262 , contains significantly more non synonymous gene mutations for proteins belonging to the categories of membrane proteins, ankyrin repeat domain-containing proteins, transcription factors and translational proteins [19]. They hypothesized that the epidemic potential of this isolate is based on modifications in the in antigenic surface proteins and growth rate, which results in a new serotype (classification of micro-organisms based on the cell surface antigens) [19]. Differences in membrane proteins or other virulence factors of $C$. burnetii can make individuals less or more susceptible for infection, in addition it could result in fewer or more clinical symptoms depending on the responsiveness of the immune system. It can be hypothesized that the C. burnetii isolates from cattle possess specific virulence factors, to which the human immune system is more profound to react. The responsiveness of the human immune system is another factor which can explain the induced cytokine differences between PBMCs stimulation with cattle isolates or goat and sheep isolates. This difference in cytokine induction may be explained by the ability of our immune system to mount an adequate pro-inflammatory response to these different strains. This was shown for Cryptococcus by Schoffelen et al. [20], as they concluded that PBMCs from healthy individuals induced a more pronounced inflammatory response against clinical heat-killed $C$. gattii isolates then to other Cryptococcus species and non-clinical C. gattii [20]. From our study we conclude that human PBMCs are able to induce a cytokine response against all $C$. burnetii isolates tested, independent the origin or MLVA genotype. There is evidence that insufficient induction of pro-inflammatory cytokines result in defective activation of the host immune response on invasion of the organism by pathogenic microbes, resulting in increased susceptibility to infections [21]. Of course, the virulence factors of the $C$. burnetii isolates and the responsiveness of the human immune system are linked to each other. The Q fever outbreak in the Netherlands can be explained by the fact that, besides possible differences in virulence of the isolate, the human immune system is not able to induce an optimal response, probably due to different antigens, leading to a higher susceptibility to infection.

IFN- $\gamma$ induced by the cattle isolates was not significantly higher than in the isolates from either goats or sheep. In contrast, the other pro-inflammatory cytokines IL-1 $\beta$ and TNF- $\alpha$ were significant higher in the cattle isolates than in the isolates from either goats or sheep. One explanation for the observed difference could be the larger variation of IFN- $\gamma$ production among the PBMCs of healthy individuals, resulting in less mutual differences between isolates of different origin (Supplement figure 2). PBMCs of 11 individuals were stimulated with the different isolates and we deliberately choose not to exclude the outliers in order to visualize the variance in cytokine responses. For example, our data show that there is a reasonable variation in IFN- $\gamma$, Il-17 and IL-22 induction per 
person. Secondly, as mentioned in the results, the general induction of IFN- $\gamma$ was much lower than for IL-1 $\beta$ and TNF- $\alpha$. Besides the significant differences in IL- $1 \beta$ and TNF- $\alpha$ induction of the cattle isolates, IL-22 was significant higher in these isolates than in the goat and sheep isolates. IL-22, in part synergistically with other cytokines such as TNF- $\alpha$, activates the expression of antimicrobial proteins like defensins. Furthermore, IL-22 promotes the production of inflammatory mediators, such as granulocyte colony-stimulating factor, IL-1 $\beta$ and lipopolysaccharide-binding protein (LBP) [22]. The initiation of these immune responses by IL-1 $\beta$, TNF- $\alpha$ and IL-22 can explain the better clearance of $C$. burnetii originating from cattle by humans.

The isolates from acute Q fever cases show a similar cytokine profile as the cattle isolates except for IL-22, even though these isolates were not retrieved from Dutch patients during the Q fever outbreak but from Kyrgyzstan, Italy and Greece. Unfortunately, there is only limited information on the background of these isolates, and we do not know whether these individuals were infected via cattle, sheep or goat. Although C. burnetii infection of human via cattle is less common, based on the cytokine patterns from our study, it is possible that these acute human isolates originated from infected cattle. The cytokine pattern of PBMCs stimulated with isolates from chronic Q fever patients was similar to that of goat isolates. Based on these findings it is possible to speculate that some C. burnetii isolates are inducing acute $\mathrm{Q}$ fever, while other $C$. burnetii isolates are responsible for chronic $\mathrm{Q}$ fever infections. This hypothesis is strengthened by several studies demonstrating that $C$. burnetii isolates originating from humans with acute Q fever differ in plasmid type [23], lipopolysaccharide profiles [24], and chromosomal DNA restriction endonuclease fragment patterns [25] from many isolates originating from chronic $\mathrm{Q}$ fever. In addition, a $28-\mathrm{kDa}$ membrane-associated protein was identified and demonstrated that the acute disease antigen A (adaA) gene is expressed in acute isolates but not carried by chronic isolates, suggesting that adaA may be a virulence factor related to acute $\mathrm{Q}$ fever [26]. In contrast, other studies indicated that no specific gene(s) on plasmids are responsible for a specific virulence phenotype [27,28]. Moreover, during the Dutch Q fever outbreak we assume that the majority of the infected individuals, both acute as chronic, are infected with the same C. burnetii MLVA genotype originating from goats. Meaning that one C. burnetii MLVA genotype can induce either acute or chronic Q fever infections in humans. In our opinion, the disease outcome of $C$. burnetii infections in humans is multi-factorial. Besides the findings presents in this study, we recently also described that single nucleotide polymorphisms in specific pattern recognition receptors increase the chance to develop chronic Q fever [29]. Taking all this into account, the severity of the infection is influenced among others by the origin and virulence of the $C$. burnetii isolate, as well as the immune status and genetic background of the infected individual. This is strengthened by the fact that many diverse C. burnetii isolates are found in goats, which are not detected in human samples. It implies that 
only the origin of the isolate does not correlate to the possibility for human infection and disease outcome.

In conclusion, in the present study we investigated the potential of $C$. burnetii isolates from different species of origin and MLVA genotypes to induce cytokine production in human PBMCs. We demonstrated that isolates derived from cattle and from acute $\mathrm{Q}$ fever patients induced higher amounts of the pro-inflammatory cytokines TNF- $\alpha$, IL- $1 \beta$ and IL-22 compared to isolates from other sources and patients with chronic Q fever. Interestingly, this could not be related to differences in MLVA genotype. The observation that cattle isolates elicit higher amounts of pro-inflammatory cytokines may explain the epidemiological observation that $\mathrm{Q}$ fever in humans is seldom related to cattle as a source for human Q fever. Although it must be noted that the all strains from acute patients were notrelated to the present outbreak. The observation that isolates from patient with acute $\mathrm{Q}$ fever induce a more pronounced pro-inflammatory cytokine response compared to patients with chronic Q fever may signify the importance of the inflammatory response to completely eradicates $C$. burnetii from the host after the initial infection in order to prevent chronic infections.

\section{Acknowledgments}

We would like to thank Anne Zeddeman for her assistance with the statistical analysis and the preparation of the dendogram. We thank Dr. Gabriela Flores-Ramirez from the Institute of Virology, Slovak Academy of Sciences in Bratislava for her help with the LPS phase determination of the $C$. burnetii strains. 


\section{References}

1. van der Hoek, W., et al., $Q$ fever in the Netherlands: an update on the epidemiology and control measures. Euro Surveill, 2010. 15(12).

2. Hogema, B.M., et al., Coxiella burnetii infection among blood donors during the 2009 Q-fever outbreak in The Netherlands. Transfusion, 2012. 52(1): p. 144-50.

3. Raoult, D., T.J. Marrie, and J.L. Mege, Natural history and pathophysiology of $Q$ fever. Lancet Infectious Diseases, 2005. 5(4): p. 219-226.

4. Maurin, M. and D. Raoult, $Q$ fever. Clin Microbiol Rev, 1999. 12(4): p. 518-53.

5. Arricau-Bouvery, N. and A. Rodolakis, Is Q fever an emerging or re-emerging zoonosis? Vet Res, 2005. 36(3): p. 327-49.

6. Georgiev, M., et al., Q fever in humans and farm animals in four European countries, 1982 to 2010. Euro Surveill, 2013. 18(8).

7. Muskens, J., et al., Prevalence of Coxiella burnetii infection in Dutch dairy herds based on testing bulk tank milk and individual samples by PCR and ELISA. Vet Rec, 2011. 168(3): p. 79.

8. Tilburg, J.J., et al., Genotyping reveals the presence of a predominant genotype of Coxiella burnetii in consumer milk products. J Clin Microbiol, 2012. 50(6): p. 2156-8.

9. Mori, M., et al., In vitro and in vivo infectious potential of coxiella burnetii: a study on Belgian livestock isolates. PLoS One, 2013. 8(6): p. e67622.

10. Roest, H.I.J., et al., Molecular Epidemiology of Coxiella burnetii from Ruminants in Q Fever Outbreak, the Netherlands. Emerging Infectious Diseases, 2011. 17(4): p. 668-675.

11. Huijsmans, C.J., et al., Single-nucleotide-polymorphism genotyping of Coxiella burnetii during a Q fever outbreak in The Netherlands. Appl Environ Microbiol, 2011. 77(6): p. 20517.

12. Hitchcock, P.J. and T.M. Brown, Morphological heterogeneity among Salmonella lipopolysaccharide chemotypes in silver-stained polyacrylamide gels. J Bacteriol, 1983. 154(1): p. 269-77.

13. Schramek, S. and C. Galanos, Lipid A component of lipopolysaccharides from Coxiella burnetii. Acta Virol, 1981. 25(4): p. 230-4.

14. Roest, H.I., et al., $Q$ fever in pregnant goats: humoral and cellular immune responses. Vet Res, 2013. 44: p. 67.

15. Roest, H.J., et al., Q fever in pregnant goats: pathogenesis and excretion of Coxiella burnetii. Plos One, 2012. 7(11): p. e48949.

16. Arricau-Bouvery, N., et al., Molecular characterization of Coxiella burnetii isolates by infrequent restriction site-PCR and MLVA typing. BMC Microbiol, 2006. 6: p. 38.

17. Oosting, M., et al., Recognition of Borrelia burgdorferi by NOD2 is central for the induction of an inflammatory reaction. J Infect Dis, 2010. 201(12): p. 1849-58. 
18. Roest, H.I., et al., Clinical microbiology of Coxiella burnetii and relevant aspects for the diagnosis and control of the zoonotic disease Q fever. Vet Q, 2013. 33(3): p. 148-60.

19. D'Amato, F., et al., The genome of Coxiella burnetii Z3055, a clone linked to the Netherlands $Q$ fever outbreaks, provides evidence for the role of drift in the emergence of epidemic clones. Comp Immunol Microbiol Infect Dis, 2014. 37(5-6): p. 281-8.

20. Schoffelen, T., et al., Cryptococcus gattii induces a cytokine pattern that is distinct from other cryptococcal species. PLoS One, 2013. 8(1): p. e55579.

21. Netea, M.G., et al., Proinflammatory cytokines and sepsis syndrome: not enough, or too much of a good thing? Trends Immunol, 2003. 24(5): p. 254-8.

22. Rutz, S., C. Eidenschenk, and W. Ouyang, IL-22, not simply a Th17 cytokine. Immunol Rev, 2013. 252(1): p. 116-32.

23. Samuel, J.E., M.E. Frazier, and L.P. Mallavia, Correlation of plasmid type and disease caused by Coxiella burnetii. Infect Immun, 1985. 49(3): p. 775-9.

24. Hackstadt, T., et al., Lipopolysaccharide variation in Coxiella burnetti: intrastrain heterogeneity in structure and antigenicity. Infect Immun, 1985. 48(2): p. 359-65.

25. Hendrix, L.R., J.E. Samuel, and L.P. Mallavia, Differentiation of Coxiella burnetii isolates by analysis of restriction-endonuclease-digested DNA separated by SDS-PAGE. J Gen Microbiol, 1991. 137(2): p. 269-76.

26. Zhang, G., et al., Identification and characterization of an immunodominant 28-kilodalton Coxiella burnetii outer membrane protein specific to isolates associated with acute disease. Infect Immun, 2005. 73(3): p. 1561-7.

27. Stein, A. and D. Raoult, Lack of pathotype specific gene in human Coxiella burnetii isolates. Microb Pathog, 1993. 15(3): p. 177-85.

28. Valkova, D. and J. Kazar, A new plasmid ( $Q p D V$ ) common to Coxiella burnetii isolates associated with acute and chronic Q fever. FEMS Microbiol Lett, 1995. 125(2-3): p. 275-80.

29. Schoffelen, T., et al., Genetic variation in pattern recognition receptors and adaptor proteins associated with development of chronic Q fever. J Infect Dis, 2015. 


\section{Supplementary figures}
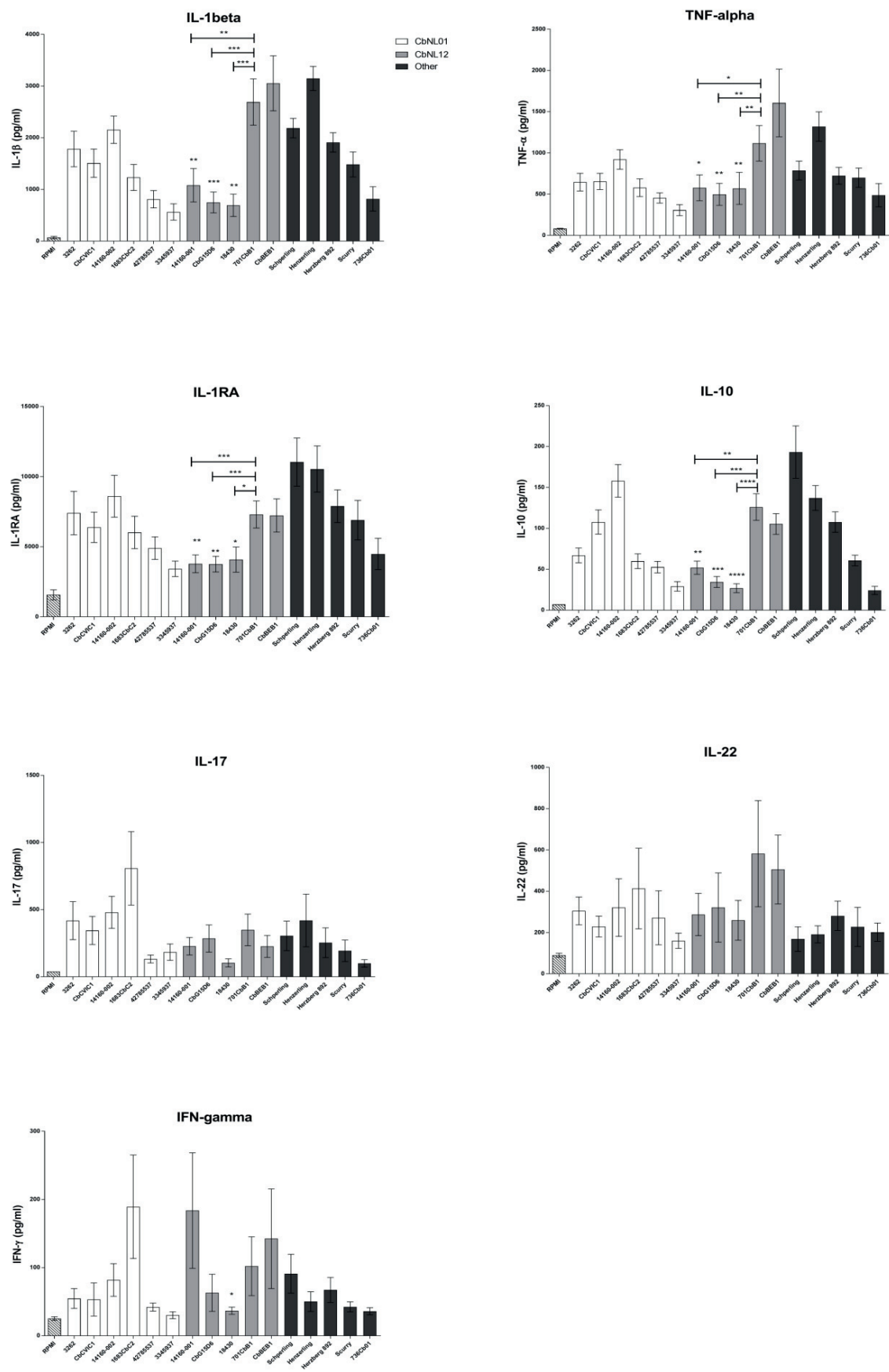
Figure S1. Cytokine profiles of the 16 different $C$. burnetii isolates based on their MLVA genotype. PBMCs of 11 healthy individuals were stimulated with RPMI medium (striped bar) and 16 different C. burnetii isolates, see Table 1 for the details of the isolates. The following cytokines were measured using ELISA: IL-1 $\beta$, TNF- $\alpha$, ILRa, IL-10, IL-17, IL-22 and IFN- $\gamma$. The color of the bars match the following isolates; white (CbNL01), grey (CbNL12), black remaining MLVA genotypes). Data is presented as mean \pm SEM. Differences between the isolates with MLVA CbNL12 were tested using the Mann-Whitney U test. The significance compared to $701 \mathrm{CbB} 1$ is indicated at the line on top of the graph, the significance compared to CbBEB1 is indicated directly on top of the corresponding bar. Only significant results are shown, ${ }^{*} \mathrm{p}<0.05, * * \mathrm{p}<0.01, * * * \mathrm{p}<0.001$, $\mathrm{p}<0.0001$. 

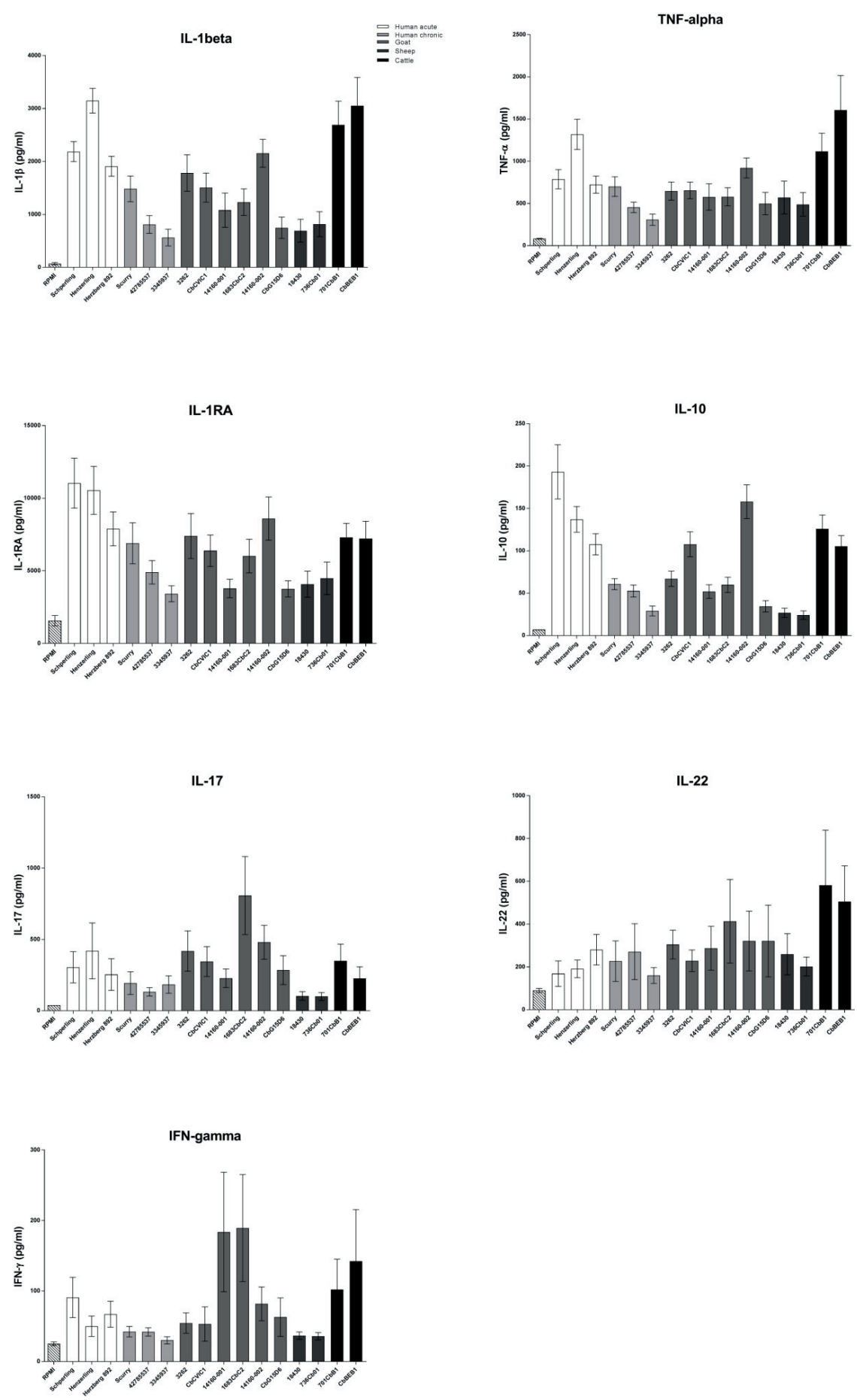
Figure S2. Cytokine profiles of the 16 different C. burnetii isolates based on their origin. PBMCs of 11 healthy individuals were stimulated with RPMI medium (striped bar) and 16 different C. burnetii isolates, see Table 1 for the details of the isolates. The following cytokines were measured using ELISA: IL-1 $\beta$, TNF- $\alpha$, IL-Ra, IL-10, IL-17, IL-22and IFN- $\gamma$. The color of the bars match the following origin; white (chronic human), light grey (acute human), medium grey (goat), dark grey (sheep), black (cattle). Data is presented as mean \pm SEM. 



\section{Chapter 8}

\section{General discussion}




\section{General Discussion}

Coxiella burnetii is an obligate intracellular bacterium that causes Q fever in animals and humans. Q fever can persist in herds of domestic ruminants causing large financial losses and outbreaks pose a major threat to public health [1,2]. During 2007-2010, a Q fever outbreak occurred in The Netherlands, where more than 40,000 people were estimated to have been infected [3]. The research described in this thesis was initiated as a response to the Dutch Q fever outbreak with the goal to characterize the $C$. burnetii strains isolated from clinical materials of infected goat, cattle, sheep and human patients [4]. The objectives were to gain a better understanding of C. burnetii virulence, diversity, physiology, and strain adaptation to various micro-environments. To this end, bacterial genomes were sequenced and comparative genomic approaches were used to identify differences in strains and their link with pathogenicity. Furthermore, we studied the diverse immunological host responses to C. burnetii strains isolated from different hosts (goat, sheep, cattle and human patients) using a mouse infection model and stimulation of healthy human PBMCs. These studies provide an overview of pathogen related factors that are potentially involved in successful infection of hosts. The studies also highlight the factors underlying the unprecedented Dutch Q fever outbreak. Finally, this thesis demonstrates the power of "Omics" approaches to study pathogens like C. burnetii, whose fastidious lifestyle makes it challenging to investigate using contemporary molecular-genetic techniques.

\section{Molecular characterization and host adaptation of $C$. burnetii strains along with factors underlying the $\mathbf{Q}$ fever outbreak}

The comparative analysis of several C. burnetii strains at the genome sequence level showed a high similarity of strains within the same genotype. Genome sequences of the Dutch CbNL12 genotype strains were most similar to the NM reference strain primarily isolated from ticks, while the genome sequences of the outbreak related CbNL01 genotype strains were more divergent. Comparative genome analysis also showed that the high virulence of the outbreak strains was not associated with acquiring novel virulence-related genes (Chapter 4 and 5). This argues against the idea that the Dutch outbreak was due to emergence of hyper-virulent $C$. burnetii strains though horizontal gene transfer of novel virulence factors $[3,5,6]$. A remarkable finding during our studies was the relatively high number of transposase genes present in the CbNL01 genotype strains. Additionally, we observed that the genome of the outbreak strains contained a large number of non-synonymous SNPs in genes encoding membrane proteins. Thus, the increased virulence/zoonotic potential of the Dutch outbreak stains is most likely due to extensive transposon-mediated genome rearrangements and alterations in the amino-acid sequence of membrane proteins (Chapter 4 and 5). 
The presence of a transposase genes (IS elements) is a unique feature of C. burnetii genomes, compared to other obligate intracellular pathogens (Rickettsia, Chlamydia, Mycobacterium leprae) and are assumed to be associated with adaptation of the pathogen to different intracellular niches [7]. These IS elements were present in the outbreak strain genomes in high numbers and were mainly associated with genome rearrangements (Chapter 5). These observations provide evidence for the notion that the high genome plasticity of the outbreak strains aid in an improved acclimatization of the bacteria in its intracellular niche. However, there is no direct evidence that such large-scale genomic rearrangements have impact on the functionalities of $C$. burnetii. On the other hand, genetic transposition events have been previously suggested to be involved in the generation of genetic variation and adaptation of other bacteria to various environments [8]. Based on the results described in this thesis and in the literature, it can be hypothesized that the large number of transposons in the outbreak strains might resulted in extensive genome rearrangements in response to environmental challenges, including those in the host. Such rearrangements can result in DNA insertions/deletions, generation of polymorphisms, pseudo-genes (genes disrupted by IS elements) or differential gene regulation favouring growth and survival of the bacteria in different niches [9-12]. Whether such genome rearrangements aid in bacterial adaptation can be tested in in vitro evolution models using nutritional deprivation conditions, which is a common condition found in the bacterial natural environment. It would not be surprising to find that transposition would be enhanced under nutritional deprived conditions to generate an array of mutants from which a fitter individual could arise, that are then enriched in the population over time due to increased growth and survival. Performing such experiments would provide more information on the evolution of virulence of $C$. burnetii strains.

Large number of mutations in membrane protein genes could also have contributed to the evolutionary success of the Dutch CbNL01 strains, as has been observed for other pathogens [13,14]. The altered membrane proteins of the Dutch outbreak strains most probably generated a distinct antigenic profile, which could have gone unrecognized by the human immune system leading to more efficient bacterial dissemination in the population. In addition, the expression of most of these mutated membrane proteins of the outbreak strains was up-regulated in vivo and few of them were among the most highly up-regulated ones (Chapter 3). This observation indicates that the altered membrane proteins are highly expressed in vivo and that they may contribute significantly to the antigenic makeup of the bacteria during infections in hosts. Hence, a change in the bacterial antigenic repertoire, qualitatively as well as quantitatively, provides a plausible explanation of the increased zoonotic potential of the outbreak strains.

The above conclusion is not in line with the results of the studies presented in Chapters 6 and 7. Although prominent genetic differences are associated with the CbNL01 genotype Dutch outbreak strains and the less prevalent CbNL12 genotype strains (Chapter 5), host immune responses were 
observed to be dependent on the origin of the C. burnetii strains, rather than on the MLVA genotype. From these findings, we anticipated (Chapter 6 and 7) that strains originating from the same host, but belonging to different genotypes, might share genetic factors or mutations in coding regions of the genomes. Such altered proteins may affect bacterial recognition by the host immune system and/or aid in escape strategies to host responses, resulting in the induction of the observed similar immune responses from strains that originate from the same host. However, from our comparative genome analysis (Chapter 5), we did not find any additional genes and/or any host-specific SNPs in coding sequences, making it less likely that strains originating from the same host (but of different genotype) share host-specific signatures.

As no host-specific genes or SNPs were identified from comparative genome analysis, other efficient evolutionary host-adaptation characteristic of $C$. burnetii strains can be predicted. All $C$. burnetii genome sequences were seen to contain many transposons (Chapter 5). It could be possible that $C$. burnetii strains undergo substantial genome rearrangements via transposition of these mobile elements. The high level of genetic diversity associated with these rearrangements could ultimately have aided adaptation to the host environment. For instance, genome analysis of the goat and human CbNL01 strains showed similar genome rearrangements relative to NM (Chapter 5). Such similarities in genome structures of these strains could have resulted in similar strategies for host adaptation and the observed similar immune responses, which is different from the immune responses induced by cattle strains (Chapter 6). Apart from changes at the genome level, it could also be that under the host influence the bacteria modifies its outer proteins/LPS coat or alters its gene regulation for efficient adaptation in hosts. For a better understanding of genome-organization and gene-regulation associated with host specificity and transmission of $C$. burnetii strains, further studies are required. Homologous and heterologous bacterial transmission studies in natural hosts, such as goats and cattle, may determine whether changes at the genome or transcriptome level aid in specialization of the bacteria in the host niche. For these experiments bacterial DNA and RNA should be directly isolated from the experimentally infected host without any sub-culturing in in vitro culturing systems. Such an approach would maintain the bacterial genome rearrangements/ transcripts in response to the residing host. These studies will help to further unravel the genetic diversity and uncover new regulatory/adaptive mechanisms employed by C. burnetii resulting in host adaptation.

Phylogenetic analysis based on genotyping $[5,15,16]$ and whole genome sequences (Chapter 5) indicate that the Dutch CbNL01 genotype strains to most likely originated from Germany and spread to the Netherlands via France [5,16]. In France and Germany, only sporadic human cases were identified pertaining to the CbNL01 genotype strains and most likely during its transmission it gained additional virulence while preserving its MLVA genotype. The high-virulent CbNL01 strains were found to be the predominant genotype causing the historically most significant Dutch outbreak of Q 
fever. Based on these findings, it can be assumed that, apart from the prominent genetic factors of the CbNL01 strains, there are other factors that orchestrated the unprecedented Dutch Q fever outbreak. One of the putative factors of the emergence of $\mathrm{Q}$ fever in Dutch dairy goat farms could be the intensive husbandry system and its proximity to the human population. This might have allowed easy transmission of the pathogen among the goat population and to humans. Secondly, as goats are seasonal breeders, abortions due to C. burnetii infections in dense goat farms cluster in time and could have increased the magnitude of environmental $C$. burnetii contamination resulting in higher risks for the emergence of human Q fever [17]. Hence, the introduction of one MLVA genotype of hypervirulent $C$. burnetii into the Dutch dairy goat population diversified over time resulting in a peak of goat abortions and leading to an enhanced scale of bacterial spreading over the country and transmissions to humans.

In conclusion, the increase in the number of $\mathrm{Q}$ fever cases in the Netherlands among humans resulted from infections with hyper-virulent CbNL01 genotype strains present in the Dutch goat population. Although, CbNL12 genotype strains (derived from goats) were not identified in humans, the ability of the strains to cause human infections should not be under-estimated for several reasons. Firstly, both CbNL01 and CbNL12 strain infections caused abortions in goats, indicating that these strains are equally virulent in goats. Moreover, the CbNL12 strain is equally virulent to other goatorigin strains of the CbNL01 genotype as measured by the mice virulence bio-assay (Chapter 2). Secondly, inadequate immune responses were generated by all goat-origin strains in mice and human PBMCs (Chapter 6 and 7). Finally, there have been reports of a few human Q fever cases in France, where the CbNL12 genotype was identified [16]. The studies from this thesis and from literature suggest that the virulence potential of $C$. burnetii strains is not only based on mutations in coding genes, but also on other host-adaptation mechanisms such as transposition or differential gene regulation and on non-genetic factors such as the risk to exposure (abortion storms in goat and sheep contrasting only mild disease in cattle). Additionally, the Dutch outbreak could have been facilitated by intensive goat farming in the affected area and its vicinity to the human populations.

\section{Host-specificity and monitoring of goat hosts to control Q fever outbreaks}

Until recently, it was not clear whether the host origin of the C. burnetii strains or the MLVA genotype is the bacterial characteristic that is associated with differences in the observed efficiency of the host immune response. Studies described in Chapters 6 and 7 emphasize that the immune responses depend largely on the host-origin of C. burnetii strains. These findings are important, as it implies that for the prevention of human Q fever, the monitoring of C. burnetii hosts is more crucial than identifying the specific bacterial genotype. The cattle origin strains induced a protective immune response in mice (Chapter 6). Similarly, it could be possible that cattle hosts provoke an efficient 
immune response upon C. burnetii infection. Such a strong immune response against cattle origin strains, directly reflects the rarely visible clinical disease and low incidence of abortions in natural cattle hosts [18]. Additionally, human PBMCs also generated a profound pro-inflammatory response upon stimulation with cattle strains (Chapter 7). This finding might explain the epidemiological observation that Q fever in humans is seldom associated with the C. burnetii infections in cattle [19]. Based on these observations, it can be presumed that $C$. burnetii strains originating from cattle hosts are well recognized by the cattle and human immune system and thereby pose a lower risk for Q fever outbreaks in humans as well as within the cattle population itself.

Host-specificity characteristics are observed in cattle-derived strains which are predominantly of the CbNL12 genotype. This genotype has rarely been found in goats [4]. It could be speculated that only a minority of bacterial infections transmitted from cattle hosts could successfully adapt in goat hosts and the majority is effectively eliminated resulting in a low prevalence of CbNL12 genotype strains in goats. The CbNL12 genotype strains has not been identified in Dutch human patients, but sporadic human cases with this CbNL12 genotype were identified in France [16]. In this case, it could be possible that humans were infected with goat-origin CbNL12 strains, but not with the CbNL12 cattle strains. This assumption is supported by the fact that human PBMCs provoked relatively high pro-inflammatory cytokine responses upon stimulation with cattle strains (CbNL12 genotype strains) and relative low responses to goat strains (both CbNL01 and CbNL12 genotype strains) (Chapter 7). Based on this finding, it can be speculated that all goat-originating strains can cause infections in humans. Additionally, insufficient immune response of humans to goat strains could enable persistent re-infections, resulting in chronic disease (Chapters 6 and 7). This indicates that goat strains might be more inclined to cause chronic infections in humans as well as in goats, which is also supported by the current literature [20,21]. Based on these immunological findings, we (Chapters 6 and 7) suggest that C. burnetii bacteria originating from goat hosts have a greater potential to cause $\mathrm{Q}$ fever outbreaks in humans. Thus, goat herds should be effectively monitored to control transmission of C. burnetii within goat population in order to reduce the risk of transmission towards humans.

An interesting question that arises from the literature is the absence of the high-virulent CbNL01 genotypes is cattle populations. It can be postulated that the CbNL01 strains cannot adapt in cattle hosts upon heterologous transmission from goats. It could also be possible that the bacteria are efficiently cleared due to prominent immune responses by cattle against CbNL01 strains. In previous genotyping studies, only 1 out of 97 genotyped cattle strains was of the CbNL01 genotype [4,22,23]. Several reasons could explain this observation. For instance; a low susceptibility of cattle to CbNL01 genotype strains or low excretion of bacteria, or the above mentioned efficient immune clearance of bacteria by host can lead to absence of the CbNL01 genotype strains in the cattle population. On the other hand, high prevalence of CbNL12 strains in cattle suggests an adaptation of this genotype in 
cattle population. Further infection experiments using natural Q fever hosts can broadly expand our knowledge of why host-specificity is seen only for a few genotypes and not for others.

\section{Improved understanding of pathogenesis of $C$ burnetii}

Since publication of the first genome sequence of a C. burnetii strain in 2003 [7], genomic approaches have emerged in Q fever studies. This has significantly contributed towards an improved knowledge of the physiology and the pathogenic abilities of the bacterium. What was lacking in our understanding of $C$. burnetii pathogenesis is a complete picture of the bacterial gene regulatory processes that occur during natural infections. Previous whole-genome expression studies of bacterial pathogens such as $V$. cholerae in experimentally infected in vivo models compared with in vitro models, has provided insights into virulence properties of the bacteria on entering the host [24]. A similar approach was used in Chapter 3, which provided an improved understanding of host-pathogen interactions of $C$. burnetii under in vivo conditions with respect to in vitro conditions. Additionally, combining both genome sequencing approaches, in order to find SNPs, and transcriptional regulation studies in mice (Chapters 3 and 5) provided further insights into virulence and adaptation mechanisms of the Dutch $C$. burnetii outbreak strains under in vivo conditions.

Notable observations from genome and transcriptome analyses (Chapters 3 and 5) were the presence of point mutations in a majority of the up-regulated membrane protein encoding genes. The potential contribution of mutations and in vivo regulation of these membrane proteins to the zoonotic potential of the outbreak strains was discussed above. The expression of a large number of DNA repair protein encoding genes and transporters (ion/proton and metabolite transporter proteins) was also upregulated under in vivo conditions, compared to in vitro culturing conditions. Around 50\% of these upregulated genes contained point mutations in the outbreak CbNL01strains. This finding is particularly interesting because up-regulation of mutated genes under in vivo conditions shows the importance of these mutations in bacterial fitness. It could be possible that up-regulation of these altered metabolite transporter proteins leads to efficient uptake of host-derived nutrients required for bacterial growth and replication in the host hostile environments. Up-regulation of altered ion/proton transporters and DNA repair proteins may enhance the capability of the bacterium in maintenance of homeostasis and cell integrity in the harsh intracellular environment. Additionally, the expression of predicted virulence related genes, encoding signal sequence-containing enzymes (acid phosphatase, phospholipases, sterol reductases) and eukaryotic-like domain containing proteins (serine/threonine protein kinases, Type IV secretion system effector proteins, ankyrin repeat containing protein), was up-regulated in vivo. This indicates that the earlier predicted virulence gene products most probably contribute to the virulence of the outbreak strains. The majority of the virulence related proteins that were up-regulated, also carried SNPs in the CbNL01 genotype strains. The presence of non-synonymous SNPs in these protein 
encoding genes which are orthologs with the NM and CbNL12 strains, indicates that these gene variants were under positive selective pressure [10]. The altered proteins in the CbNL01 genotype strains might play a role in the manipulation of host components or host intracellular processes contributing to successful bacterial infection and persistence in host cells.

Until now transcriptional studies on C. burnetii were only performed on in vitro cultured cells and mostly focused on a few target genes $[25,26]$. Despite the value of in vitro studies, these models cannot easily reproduce the complex and dynamic environment that $C$. burnetii is exposed to under in vivo conditions. Hence, the study as described in Chapter 3 is the first report on C. burnetii genomewide gene expression patterns in an in vivo model simulating a natural infection. The study clearly revealed up-regulation of a larger number of genes for bacterial survival/growth under in vivo conditions, than for in vitro conditions. Further studies involving the generation of knockout mutants of the up-regulated genes would be crucial to assess whether absence of these gene products would influence bacterial growth and verify their role in virulence of $C$. burnetii. Additionally, the present transcriptome results are based on high-virulent CbNL01 strains. Our previous mouse virulence bioassays (Chapter 2 and unpublished data) showed that several strains exhibited low-virulence characteristics. A comparison of gene regulation in in vivo hosts of high and low virulent $C$. burnetii strains would contribute to our understanding of pathogenic mechanisms and virulence.

\section{Insights from genomics into the large Dutch $Q$ fever outbreak}

\section{Confirming the source of the Dutch $Q$ fever outbreak}

Molecular epidemiological/genotyping studies strongly suggested that goats were the primary source of the Dutch human Q fever outbreak from 2007-2010 [4,16,27]. Although genotyping approaches are widely used for outbreak investigations, these methods sometimes lack sufficient discriminatory power as it evaluates only a few genome loci. Besides, the development of high-throughput whole genome sequencing techniques (WGS) has revolutionized the conduct of outbreak investigation. Because of its detailed level of resolution, the WGS techniques could augment the traditional approaches leading to more accurate and efficient outbreak investigations. Comparison of the CbNL01 genotype veterinary and human strain genome sequences showed large genome sequence similarities with minimal genetic differences (few point mutations only). Based on these results, the studies in Chapters 4 and 5 confirmed the conclusions from previous epidemiological and genotypic studies, linking goat and human infections during the large Dutch Q fever outbreak.

Genome sequencing during outbreaks of infectious diseases is fundamentally useful to understand the pathogen evolution and molecular epidemiology. However, such information should become available rapidly in order to guide the disease control measures. Recently, with the largest Ebola outbreak, real-time WGS samples from Ebola patients provided very detailed information about 
how cases were related. The determined phylodynamics provided important clues to successfully halt the transmission of the virus [28]. Another example is the recent avian influenza H5N8 outbreak in The Netherlands where WGS and phylodynamics provided invaluable insights that helped to efficiently manage the disease outbreak $[29,30]$. Real-time outbreak surveillance at the genome sequence level is now possible and has been used in several other outbreak cases in recent years $[31,32]$ and has proved to have a broad impact on public health. The development of a fully automated bioinformatics pipeline for WGS data analysis of C. burnetii is much needed to make this powerful tool more widely accessible for future real-time investigations of $\mathrm{Q}$ fever outbreaks.

\section{Phylogenetic diversity of Dutch C. burnetii strains}

The phylogenome analysis of a large number of strains from different geographical areas has helped to reconstitute the epidemiology of $C$. burnetii strains from one region to another as described above for Dutch outbreak CbNL01 genotype strains [5,16]. Furthermore, Chapter 5 also showed a predominant clustering of genome sequences of the CbNL12 genotype strains isolated from cattle in The Netherlands and France. Similar observations were seen in C. burnetii genotyping studies, which showed the presence of CbNL12 genotypes in consumer cow milk products obtained from various European countries [23]. Thus, phylogenetics implies a clonal spread and host-specificity of CbNL12 strains among the European cattle population.

Although WGS and genotyping approaches showed similar results with respect to Dutch Q fever outbreak, analytically WGS offers superior resolution of strain types and is less prone to falsepositive/ negatives. Further, genomic differences distinguishing strains can be precisely measured, allowing high-resolution inference of phylogenomic relationships and of the sequence of transmission events. Given these results, the WGS should replace traditional methods as the gold standard method for bacterial strain typing in molecular epidemiology applications and outbreak investigations.

\section{Culturing and infection models of $C$. burnetii}

In vitro model for cell-free culture of $\mathrm{C}$. burnetii

For long time, in vitro growth of $C$. burnetii was limited to the use of mammalian cell cultures or embryonated eggs [33,34]. This changed when the host cell-free medium became available for in vitro growth of $C$. bunetii. This advanced medium is designed such that the metabolic requirements of $C$. burnetii are fully met, allowing its growth in liquid medium and as "colonies" in top-agar plates [35,36]. In Chapters 2, 4 and 5 the successful growth of C. burnetii was demonstrated for several strains. The reference NM strain grew rapidly in this new medium with optimal bacterial yields, while the Dutch field strains were more difficult to grow. The initial cultures of the Dutch field strains had longer lag phases than the reference strain, although this improved with repeated culture. From these 
observations, it can be assessed that different C. burnetii strains differ in their adaptation capabilities to new environmental conditions but eventually adapt to the prevailing conditions and thrive in the environment it resides in. Once adapted, the virulence characteristics of these strains are similar to cell-based cultured strains of similar passage number (Chapter 2). Moreover, at the transcriptome level we observed that the gene expression profiles of bacteria from the first two serial passages from cellcultures to cell-free cultures were very similar to completely adapted cell-free cultured strains (Chapter 3). This suggested rapid adaptation capabilities of $C$. burnetii when moved from one environment to another. Overall, robust growth of several C. burnetii strains was observed in the liquid cell-free medium. However, colony formation in agarose plates was successful only for the reference NM strain which is also widely shown by other research groups [36,37], but not for other field strain. For the field strains no correlations of colony forming units (CFU) with focus forming units (FFU) was observed. This demonstrates that more research is needed to generate a reliable plating method for laboratory and field strains. Further improvement of the plating method for C. burnetii is necessary for development of a fully axenic system for genetic transformation and clone selection.

In Chapter 2, similar virulence parameters of C. burnetii strains propagated from cell-free cultures and in vivo mimicking cell-based culture systems were observed. This suggests that $C$. burnetii propagated in the advanced cell-free system maintain their virulence associated characteristics. Thereby, the cell-free culture system is a valuable tool to study the pathogen by using molecular-techniques. Such techniques are much more difficult to apply when cell-based growth systems are used. Additionally, the axenic system will aid in development of Q fever preventatives, such as subunit vaccines as well as production of recombinant antigens. In Chapters 4 and 5, we have also used this cell free system to obtain pure bacterial DNA for WGS studies. Finally, a similar strategy used to establish cell-free culture conditions for $C$. burnetii was applicable for the development of a axenic culture system for Chlamydia [38]. Therefore such an approach could also be applicable to support the growth of other medically important obligate intracellular pathogens.

\section{Infection model for $\mathrm{C}$. burnetii}

All our animal experiments were conducted with a newly developed mice model using "Swiss OF1 mice". This model is currently being used by several other research groups for evaluating pathogenicity of C. burnetii with consistent outcomes [39]. In Chapter 2 we observed similar relative virulence of several cell-cultured strains from two independent animal experiments. This observation further underlines the reproducibility of these virulence associated measurements of C. burnetii in the developed mice model. Consistent with previous studies [34], the Swiss OF1 mice likewise showed early and strong systemic antibody responses to several C. burnetii strains. The observed kinetics of IgM and IgG antibody's in mice against phase I and II antigens were also in line with previous 
published work in goats [40] and humans [41], strengthening the Swiss OF1 mice as a valid experimental model for immunological studies on Q fever agents (Chapter 6). Additionally, gene expression profiles of $C$. burnetii outbreak strains isolated from mice spleen and from goat aborted placenta were similar to each other (unpublished data). Such similar gene expression profiles of bacteria obtained from natural hosts and the experimental infection mice model further emphasizes the relevance of mice infection models in the study of $\mathrm{Q}$ fever.

\section{Tools and approaches to study $C$. burnetii pathogen}

\section{Enumeration of live bacteria}

As colony counting in top-agar plates was not suitable for all C. burnetii strains, we searched for alternative methods to assess bacterial viability. One way to achieve this is by quantification of mRNA transcripts. However, the correlation between the occurrence of mRNA and viability is not necessarily always well-defined. This is because, mRNA synthesis might indicate the metabolic activity of cells rather than viability, which diminishes the power of this method [42]. Alternately, cell viability can be evaluated by indirect measurements of the state of the cells, such as membrane integrity. Often dual fluorescence staining kits are used for this purpose requiring use of a flow cytometer or fluorescent microscope [43]. The high cost of these instruments means they are not always available in level 3 bio-safety laboratories. To circumvent these constraints, a simple method was designed which distinguished live and dead bacterial cells, based on the same principle of cell membrane integrity, by using a propidium monoazide (PMA) dye. The PMA dye selectively penetrates only into dead bacterial cells with compromised membranes and intercalates with DNA, inhibiting its amplification in a subsequent qPCR step [44]. Such a system is for instance used in the detection of Legionella in drinking and sanitary water systems, where detection of live/dead bacteria is crucial $[44,45]$. The PMA technique was applicable to $C$. burnetii obtained from both cell-based and cell-free systems. The live bacterial copies assessed by PMA-PCR were similar to bacterial counts obtained from laborious traditional methods such as FFU [46] and CFU [36], validating the efficiency of PMA-PCR technique. This safe and feasible PMA-PCR method allowed the number of living organisms to be calculated in the inocula used for in vitro and in vivo infections, with reproducible outcomes (Chapters 2 and 6).

\section{Mechanism of phase variation and its immunological outcomes}

Phase variation is a well-recognized phenomenon in C. burnetii. Although, bacterial culture in in vitro systems is the only well-defined factor known to influence phase variation. The precise mechanism of phase variation, resulting in truncation of LPS, is still unknown. Deletion of (part of) cluster of Oantigen genes (CBU0676 to CBU706) which are implicated in the synthesis of the terminal part of LPS, is generally thought to be associated with phase variation [47]. Transcriptome and genome 
sequencing studies conducted in Chapters 2 and 5 significantly contributed to the knowledge of the mechanism of phase variation. The general observations from these studies were that deletion of genome region encoding the $\mathrm{O}$-antigen genes ( $38 \mathrm{~kb}$ size) occurs with increasing passages in in vitro culture models. All CbNL01 sequenced strains showed a deletion of a portion of the CBU0691 gene. The expression of this truncated gene was also downregulated in a highly-passaged CbNL01 genotype strain (602). This deletion could have a significant effect on LPS synthesis as it is implicated in virenose synthesis, a unique component of the C. burnetii phase I structure. Such a deletion event in the CBU0691 gene of all CbNL01 strains could be the starting point for phase variation. Moreover, in low passaged NLhu3345937 strain of CbNL01 genotype, a deletion of several genes at the beginning of the O-antigen gene cluster (CBU0676 to CBU0682) was also observed. These genes were also deleted in a high passaged 602 strain (Chapter 2), but not in low passaged 602 strain (Chapter 5). Combining these results, it can be speculated that the possible mechanism of excision of O-antigen genes in CbNL01 strains is as follows: the process is initiated by the deletion of a portion of the CBU0691 gene followed by deletions of O-antigen genes in the beginning of the LPS gene cluster (CBU0676 to CBU0682), deletion further progresses till the gene CBU0697 at the end of the Oantigen cluster as seen in high passaged 602 strain (Chapter 2). Additionally, genome sequences of all CbNL12 strains showed only one deletion of 201 bp within gene CBU0686. These consistent partial gene deletions in CbNL01 and CbNL12 strains suggest a genotype-specific phase shifting of $C$. burnetii strains. Moreover, deletions were not observed in the other genotypes strains, but only in CbNL01 and CbNL12 genotype strains, although a low passage numbers were used for analyses of $C$. burnetii strains. Based on these findings, it can be speculated that CbNL01 and CbNL12 show a higher rate of phase variation compared to the other genotype strains.

Immunological studies upon $C$. burnetii infection in mice, guinea pigs and the natural hosts goats and humans showed an early antibody response against phase II antigens rather than phase I antigens, though the infecting strains contained a phase I LPS (Chapter 6) [40,41,48,49]. There is no good explanation for such a typical phase II response prior to phase I responses. It could be possible that the primary phase II response, demonstrated to be a non-protective response [50], could act as an immunological decoy tactic to avoid/ delay responses against phase I antigens that efficiently neutralize the pathogen. Previous studies have provided clues that the delayed phase I antibody response are due to shielding of toll-like receptor ligands by LPS [50]. Whereas, the early phase II antibody responses are generated against easily accessible surface proteins that are shared in both phase I and II bacteria [51]. To this end, the existing literature does not comprise studies focusing on the advantages of an early phase II antibody response for a phase I bacteria. Further studies on identification of components resulting in the generation of phase II antibodies in a phase I LPS 
containing bacteria and the role of phase II antibody in overall bacterial protection, will be crucial to gain a better understanding of $C$. burnetii infection process and survival in hosts.

\section{Recommendations and future scientific perspectives}

The studies presented in this thesis provide the results of genomic and transcriptomic studies to investigate the genetic potential of $C$. burnetii and provides more information on virulence, diversity and differential immune responses against various $C$. burnetii infections. The results described in this thesis contribute to a better explanation of the large Dutch Q fever outbreak and improve our understanding of bacterial adaptations, pathogenesis as well as genotype-phenotype relationships of $C$. burnetii strains. Furthermore, these results provide a framework for future studies and recommendations for the development of vaccines and diagnostic tools for $\mathrm{Q}$ fever.

The problem with the current $C$. burnetii vaccines used in goats, is that after vaccination the bacteria are not completely eliminated from the host, although the clinical symptoms such as abortions and bacterial shedding are significantly decreased [52]. Since the current vaccines are prepared from the NM phase I strain, it might not represent the optimal antigenic repertoire of goat strains. The mutations in several membrane proteins of goat strains relative to the NM strain strengthen this (Chapter 5). The transcriptome study of a goat strain has shown up-regulation of several membrane associated proteins, including the mutated ones, under in vivo conditions (Chapter 3). Based on these findings, membrane proteins could potentially qualify as vaccine candidates and should be further tested for antigenicity and their potential for protection. Subunit vaccines prepared from these (mutated) surface proteins might confer improved protection to goats. Antigenicity of these surface associated proteins can be assessed by a protein array approach using convalescent sera generated from infected goats. Such an approach would be a robust way to assess large-scale identification of $C$. burnetii specific antibodies in sera. Proteins which are recognized by antibodies should be further checked to see if these purified proteins can elicit strong antibody response and confer protection in mice and ultimately in natural goat hosts against $C$. burnetii infection. Additionally, differentiation of infected and vaccinated animals is not possible based on the current phase I Q fever vaccines. This is a major drawback because the immune response generated by the current vaccines cannot be discriminated from the immune response from animals that have been exposed to a natural infection. Thus, development of specific vaccines such as DIVA (Differentiating Infected from Vaccinated Animals) vaccines that can distinguish infected and vaccinated animals could be a possible solution. Such vaccines will successfully allow treatment of infected animals differently from vaccinated animals

The studies in this thesis demonstrated that human PBMCs respond differently to strains obtained from acute and chronic infected patients. A low inflammatory cytokine response is generated 
by human PBMCs upon stimulation with strains isolated from chronic patients and significantly higher response is generated against strains isolated from acute patients (Chapter 7). This indicates that the human acute and chronic origin strains might express different antigens or confer a differential transcriptional response in the host during acute and chronic infections. Such information can be used for vaccine development and/ or therapeutic treatments directed towards prevention of bacterial resistance to host response and its persistent re-infections.

A final recommendation concerns the presence of around 33\% of hypothetical genes in the genome of C. burnetii emphasizing a large gap in our knowledge on the functional properties of this pathogen. Additionally, we observed that these genes contained a large number of mutations in the outbreak strains and that they are differentially regulated under in vivo conditions, compared to in vitro conditions (Chapters 5 and 3). This highlights the potential contribution of these hypothetical genes in unique lifestyle and/or pathogenic potential of C. burnetii. Improved functional annotation of these genes is crucial for further a better understanding of how this pathogen hides/resides in its natural host. Elucidating the function of unknown genes is one the biggest challenges of the post-genomic era especially for bacteria that cannot be easily cultured in vitro. 


\section{References}

1. Maurin M, Raoult D. Q fever. Clin. Microbiol. Rev. 1999;12:518-53.

2. Porter SR, Czaplicki G, Mainil J, Guattéo R, Saegerman C. Q Fever: current state of knowledge and perspectives of research of a neglected zoonosis. Int. J. Microbiol. 2011;2011:248418.

3. Roest HIJ, Tilburg JJHC, van der Hoek W, Vellema P, van Zijderveld FG, Klaassen CHW, et al. The Q fever epidemic in The Netherlands: history, onset, response and reflection. Epidemiol. Infect. 2011;139:1-12.

4. Roest HIJ, Ruuls RC, Tilburg JJHC, Nabuurs-Franssen MH, Klaassen CHW, Vellema P, et al. Molecular epidemiology of Coxiella burnetii from ruminants in Q fever outbreak, the Netherlands. Emerg. Infect. Dis. 2011;17:668-75.

5. Tilburg JJHC, Rossen JWA, van Hannen EJ, Melchers WJG, Hermans MHA, van de Bovenkamp J, et al. Genotypic diversity of Coxiella burnetii in the 2007-2010 Q fever outbreak episodes in The Netherlands. J. Clin. Microbiol. 2012;50:1076-8.

6. Enserink M. Questions Abound in Q-Fever Explosion in the Netherlands. Science. 2010;327:266-7.

7. Seshadri R, Paulsen IT, Eisen JA, Read TD, Nelson KE, Nelson WC, et al. Complete genome sequence of the Q-fever pathogen Coxiella burnetii. Proc. Natl. Acad. Sci. U. S. A. 2003;100:545560 .

8. Hall BG. Is the occurrence of some spontaneous mutations directed by environmental challenges? New Biol. 1991;3:729-33.

9. Pallen MJ, Wren BW. Bacterial pathogenomics. Nature. 2007;449:835-42.

10. Beare PA, Unsworth N, Andoh M, Voth DE, Omsland A, Gilk SD, et al. Comparative genomics reveal extensive transposon-mediated genomic plasticity and diversity among potential effector proteins within the genus Coxiella. Infect. Immun. 2009;77:642-56.

11. Beare PA, Samuel JE, Howe D, Virtaneva K, Porcella SF, Heinzen RA. Genetic Diversity of the Q Fever Agent, Coxiella burnetii, Assessed by Microarray-Based Whole-Genome Comparisons. J. Bacteriol. 2006;188:2309-24.

12. Rohmer L, Fong C, Abmayr S, Wasnick M, Larson Freeman TJ, Radey M, et al. Comparison of Francisella tularensis genomes reveals evolutionary events associated with the emergence of human pathogenic strains. Genome Biol. 2007;8:R102.

13. Grodeland G, Mjaaland S, Roux KH, Fredriksen AB, Bogen B. DNA vaccine that targets hemagglutinin to MHC class II molecules rapidly induces antibody-mediated protection against influenza. J. Immunol. Baltim. Md 1950. 2013;191:3221-31.

14. Wu Z, Periaswamy B, Sahin O, Yaeger M, Plummer P, Zhai W, et al. Point mutations in the major outer membrane protein drive hypervirulence of a rapidly expanding clone of Campylobacter jejuni. Proc. Natl. Acad. Sci. U. S. A. 2016;113:10690-5.

15. D'Amato F, Eldin C, Raoult D. The contribution of genomics to the study of Q fever. Future Microbiol. 2016;11:253-72.

16. Tilburg JJHC, Roest H-JIJ, Buffet S, Nabuurs-Franssen MH, Horrevorts AM, Raoult D, et al. Epidemic Genotype of Coxiella burnetii among Goats, Sheep, and Humans in the Netherlands. Emerg. Infect. Dis. 2012;18:887-9. 
17. Brom RV den, Vellema P. Q fever outbreaks in small ruminants and people in the Netherlands. Small Rumin. Res. 2009;86:74-9.

18. Baca OG, Paretsky D. Q fever and Coxiella burnetii: a model for host-parasite interactions. Microbiol. Rev. 1983;47:127-49.

19. Georgiev M, Afonso A, Neubauer H, Needham H, Thiery R, Rodolakis A, et al. Q fever in humans and farm animals in four European countries, 1982 to 2010. Euro Surveill. Bull. Eur. Sur Mal. Transm. Eur. Commun. Dis. Bull. 2013;18.

20. Berri M, Rousset E, Champion JL, Russo P, Rodolakis A. Goats may experience reproductive failures and shed Coxiella burnetii at two successive parturitions after a Q fever infection. Res. Vet. Sci. 2007;83:47-52.

21. Mazokopakis EE, Karefilakis CM, Starakis IK. Q fever endocarditis. Infect. Disord. Drug Targets. 2010;10:27-31.

22. Roest HIJ, van Solt CB, Tilburg JJHC, Klaassen CHW, Hovius EK, Roest FTF, et al. Search for possible additional reservoirs for human Q fever, The Netherlands. Emerg. Infect. Dis. 2013;19:834-5.

23. Tilburg JJHC, Roest HJIJ, Nabuurs-Franssen MH, Horrevorts AM, Klaassen CHW. Genotyping reveals the presence of a predominant genotype of Coxiella burnetii in consumer milk products. J. Clin. Microbiol. 2012;50:2156-8.

24. Xu Q, Dziejman M, Mekalanos JJ. Determination of the transcriptome of Vibrio cholerae during intraintestinal growth and midexponential phase in vitro. Proc. Natl. Acad. Sci. U. S. A. 2003;100:1286-91.

25. Morgan JK, Luedtke BE, Thompson HA, Shaw EI. Coxiella burnetii type IVB secretion system Region I genes are expressed early during infection of host cells. FEMS Microbiol. Lett. 2010;311:619.

26. Coleman SA, Fischer ER, Howe D, Mead DJ, Heinzen RA. Temporal Analysis of Coxiella burnetii Morphological Differentiation. J. Bacteriol. 2004;186:7344-52.

27. Van Steenbergen JE, Morroy G, Groot C a. R, Ruikes FGH, Marcelis JH, Speelman P. An outbreak of Q fever in The Netherlands--possible link to goats. Ned. Tijdschr. Geneeskd. 2007;151:1998-2003.

28. Quick J, Loman NJ, Duraffour S, Simpson JT, Severi E, Cowley L, et al. Real-time, portable genome sequencing for Ebola surveillance. Nature. 2016;530:228-32.

29. Bouwstra RJ, Koch G, Heutink R, Harders F, van der Spek A, Elbers AR, et al. Phylogenetic analysis of highly pathogenic avian influenza $\mathrm{A}(\mathrm{H} 5 \mathrm{~N} 8)$ virus outbreak strains provides evidence for four separate introductions and one between-poultry farm transmission in the Netherlands, November 2014. Euro Surveill. Bull. Eur. Sur Mal. Transm. Eur. Commun. Dis. Bull. 2015;20.

30. Bouwstra R, Heutink R, Bossers A, Harders F, Koch G, Elbers A. Full-Genome Sequence of Influenza A(H5N8) Virus in Poultry Linked to Sequences of Strains from Asia, the Netherlands, 2014. Emerg. Infect. Dis. 2015;21:872-4.

31. Le VTM, Diep BA. Selected Insights from Application of Whole Genome Sequencing for Outbreak Investigations. Curr. Opin. Crit. Care. 2013;19:432-9. 
32. Jackson BR, Tarr C, Strain E, Jackson KA, Conrad A, Carleton H, et al. Implementation of Nationwide Real-time Whole-genome Sequencing to Enhance Listeriosis Outbreak Detection and Investigation. Clin. Infect. Dis. Off. Publ. Infect. Dis. Soc. Am. 2016;63:380-6.

33. Roest H-J, van Gelderen B, Dinkla A, Frangoulidis D, van Zijderveld F, Rebel J, et al. Q Fever in Pregnant Goats: Pathogenesis and Excretion of Coxiella burnetii. PLoS ONE. 2012;7:e48949.

34. Mori M, Boarbi S, Michel P, Bakinahe R, Rits K, Wattiau P, et al. In Vitro and In Vivo Infectious Potential of Coxiella burnetii: A Study on Belgian Livestock Isolates. PLoS ONE. 2013;8:e67622.

35. Omsland A, Cockrell DC, Howe D, Fischer ER, Virtaneva K, Sturdevant DE, et al. Host cell-free growth of the Q fever bacterium Coxiella burnetii. Proc. Natl. Acad. Sci. 2009;106:4430-4.

36. Omsland A, Beare PA, Hill J, Cockrell DC, Howe D, Hansen B, et al. Isolation from Animal Tissue and Genetic Transformation of Coxiella burnetii Are Facilitated by an Improved Axenic Growth Medium ${ }^{\nabla}$. Appl. Environ. Microbiol. 2011;77:3720-5.

37. Weber MM, Chen C, Rowin K, Mertens K, Galvan G, Zhi H, et al. Identification of Coxiella burnetii Type IV Secretion Substrates Required for Intracellular Replication and Coxiella-Containing Vacuole Formation. J. Bacteriol. 2013;195:3914-24.

38. Omsland A, Sager J, Nair V, Sturdevant DE, Hackstadt T. Developmental stage-specific metabolic and transcriptional activity of Chlamydia trachomatis in an axenic medium. Proc. Natl. Acad. Sci. U. S. A. 2012;109:19781-5.

39. Rodolakis A. Q fever in France, International Q fever conference. Breda, Netherlands; 2010.

40. Roest HIJ, Post J, van Gelderen B, van Zijderveld FG, Rebel JMJ. Q fever in pregnant goats: humoral and cellular immune responses. Vet. Res. 2013;44:67.

41. Dupuis G, Péter O, Peacock M, Burgdorfer W, Haller E. Immunoglobulin responses in acute Q fever. J. Clin. Microbiol. 1985;22:484-7.

42. Keer JT, Birch L. Molecular methods for the assessment of bacterial viability. J. Microbiol. Methods. 2003;53:175-83.

43. Stiefel P, Schmidt-Emrich S, Maniura-Weber K, Ren Q. Critical aspects of using bacterial cell viability assays with the fluorophores SYTO9 and propidium iodide. BMC Microbiol. [Internet]. 2015 [cited 2016 Dec 2];15. Available from: http://www.ncbi.nlm.nih.gov/pmc/articles/PMC4337318/

44. Yáñez MA, Nocker A, Soria-Soria E, Múrtula R, Martínez L, Catalán V. Quantification of viable Legionella pneumophila cells using propidium monoazide combined with quantitative PCR. J. Microbiol. Methods. 2011;85:124-30.

45. Kirschner AKT. Determination of viable legionellae in engineered water systems: Do we find what we are looking for? Water Res. 2016;93:276-88.

46. Stein A, Louveau C, Lepidi H, Ricci F, Baylac P, Davoust B, et al. Q Fever Pneumonia: Virulence of Coxiella burnetii Pathovars in a Murine Model of Aerosol Infection. Infect. Immun. 2005;73:246977 .

47. Hoover TA, Culp DW, Vodkin MH, Williams JC, Thompson HA. Chromosomal DNA deletions explain phenotypic characteristics of two antigenic variants, phase II and RSA 514 (crazy), of the Coxiella burnetii nine mile strain. Infect. Immun. 2002;70:6726-33. 
48. Williams JC, Thomas LA, Peacock MG. Humoral immune response to Q fever: enzyme-linked immunosorbent assay antibody response to Coxiella burnetii in experimentally infected guinea pigs. J. Clin. Microbiol. 1986;24:935-9.

49. Kishimoto RA, Rozmiarek H, Larson EW. Experimental Q fever infection in congenitally athymic nude mice. Infect. Immun. 1978;22:69-71.

50. Shannon JG, Howe D, Heinzen RA. Virulent Coxiella burnetii does not activate human dendritic cells: Role of lipopolysaccharide as a shielding molecule. Proc. Natl. Acad. Sci. U. S. A. 2005;102:8722-7.

51. Hackstadt T. Steric hindrance of antibody binding to surface proteins of Coxiella burnetti by phase I lipopolysaccharide. Infect. Immun. 1988;56:802-7.

52. de Cremoux R, Rousset E, Touratier A, Audusseau G, Nicollet P, Ribaud D, et al. Assessment of vaccination by a phase I Coxiella burnetii-inactivated vaccine in goat herds in clinical Q fever situation. FEMS Immunol. Med. Microbiol. 2012;64:104-6. 

Summary 


\section{Summary}

The largest reported Q fever outbreak in The Netherlands has resulted in a serious burden of disease in humans and mass culling of infected goats. In order to minimize such complications in the future, it is imperative to have a thorough understanding of the disease causing pathogen and development of effective $\mathrm{Q}$ fever vaccines. The research described in this thesis focuses on the molecular characterization of $C$. burnetii outbreak strains from infected goats, cattle, sheep and humans using pathogen-omics approaches. Our studies were initialized to better understand the bacterial pathogenesis and identify pathogen related factors that has modulated the disease outbreak. We also examined the differential immune responses generated against various C. burnetii strains derived from a variety of hosts in different model-systems. These experiments were conducted to provide an immunological explanation, why C. burnetii strains from goats are associated with high virulence and large disease outbreaks, contrasting the strains found in cattle. The results in this thesis expand our knowledge regarding pathogen associated molecular and immunological factors involved in host infections.

Chapter 1, as an introduction provides the necessary background information of the Q fever disease and its causative agent $C$. burnetii, to offer a better understanding of the studies presented in this thesis. The Q fever outbreak in the Netherlands, tools used for outbreak investigation and control measures taken to limit the disease in humans are explained in detail, which provides the basis of the conducted research. In addition, this chapter also briefly introduces the fast advancements in molecular $\mathrm{Q}$ fever research in the recent years, while pinpointing some of the missing gaps that needs to be addressed. This is vital in order to obtain a further improved knowledge of the pathogen, which can aid in prevention of future $\mathrm{Q}$ fever outbreaks using better diagnostics or vaccines designed at the molecular level.

The first publication of C. burnetii complete genome in 2003 has significantly improved the knowledge on the metabolic requirements of the pathogen. This has led to the development of an axenic medium, which allowed propagation of $C$. burnetii outside host cells. As studies of pathogenicity and molecular engineering can benefit from this system, in Chapter $\mathbf{2}$ we checked if $C$. burnetii strains virulence phenotype is consistently maintained in the cell-free system compared to the gold standard cell-based culturing system. These experiments were conducted in order to confirm that this advanced culture system maintains the virulence characteristics of the bacteria and not lead to additional culture-specific adaptations. We assessed the viability of $C$. burnetii by a propidium monoazide (PMA) treatment based real time PCR and determined expression of LPS encoding genes of bacteria in both culture systems as key parameters of its infection potential. We also used a mice infection model to directly assess the virulence potential of the strains propagated from both these systems using the degree of splenomegaly and bacterial load in spleen (accepted virulence associated 
measurements). Our studies showed similar virulence phenotype of strains propagated from both culture systems confirming that the cell-free culture system itself does not have a significant influence on virulence phenotype of $C$. burnetii strains compared to cell-cultures. Additionally, a high passaged cell-free cultured strain showed LPS modification from phase I to phase II forms, which is also characteristically seen upon serial passage of C. burnetii in other in vitro models (embryonated eggs and cell cultures).

As an obligate intracellular pathogen, $C$. burnetii requires metabolic regulation such that it can adapt to the intracellular environment of the host cell. In Chapter 3 we assessed the complete transcriptional profile of $C$. burnetii in vivo during infection (mice spleen) and in in vitro models (cell and cell-free cultures) to assess differential gene regulation of the bacterium in different microenvironments. Between different models we observed major differential regulation of the bacteria in metabolic pathways and virulence associated genes. Under in vivo conditions, the prominent findings from our study include the enhanced expression of anionic cardiolipins and ion/proton exchangers for $\mathrm{pH}$ homeostasis and survival of the pathogen within the highly acidic vacuoles of host cells; enhanced organic molecule transportation indicating bacterial dependence on host products; anaerobic energy metabolism and enhanced ROS scavenging enzymes/ DNA repair mechanisms to combat against oxidative stress in host cells. The in vivo environment also significantly induced expression of several known virulence genes in C. burnetii, including those implicated in LPS synthesis, colonization and factors predicted to be associated with host-cell modulations (inhibition of apoptosis, mimicking host proteins). This indicates that the virulence gene products most probably contribute to the bacterial adaptation and survival under in vivo conditions. The present study improved our knowledge on bacterial adaptation in host-specific environments and provided a framework for identification of virulence factors important for the design of vaccines.

In Chapter 4 and 5 we determined and fully reconstructed the genome sequences of $C$. burnetii strains collected from goats, sheep and cattle during Dutch Q fever outbreak period. Additional strains obtained from Dutch chronic Q fever patients were also included. In these studies we used comparative bioinformatics approaches and identified highly similar genome sequences of outbreak CbNL01 genotype goat and human strains. These findings confirmed the previous epidemiological and genotypic studies linking goats as source of human infections during the Dutch Q fever outbreak. Furthermore, our phylogenetic analysis of sequenced strains along with all published genome sequences of $C$. burnetii showed a genotype-specific clustering of genomes indicating similar genome sequences of strains within one MLVA genotypic group. Hence, our studies additionally validated genotyping tools (MLVA and MST) as good representations of full genome diversity. Even though Whole Genome Sequencing might be preferred in the future, MLVA and MST provided invaluable insights for outbreak investigations and molecular epidemiology of C. burnetii during the 
Dutch Q fever outbreak. We further assessed genetic differences in outbreak CbNL01 strains compared to the reference Nine Mile (NM) and CbNL12 genome sequences. We found major differences predominantly as point mutations and increase in number of transposase genes in outbreak strain. Non-synonymous point mutations were found in several membrane proteins, DNA repair genes, genes involved in translation, transporters, Type IV secretion system and potential virulence factors. Mutations in genes encoding membrane proteins which were orthologs with the NM and CbNL12 strains, indicates that these genes are under selective pressure to vary antigenically. While mutations in other genes could be possibly involved in alter of protein synthesis, protein export abilities as well as increase in virulence potential. Moreover, the large number of transposons resulted in increased genome plasticity of outbreak strains compared to other strains. Such movement of mobile elements could have a major influence on $C$. burnetii's genome evolution and function by aiding in improved acclimatization of the bacteria in its intracellular niche. These variations in critical genes of C. burnetii and extensive transposon-mediated genome rearrangements could have led to the increased epidemic potential of CbNL01 strains.

In Chapter 6 and 7 we used the strains described in Chapter 5 to identify if differences in host immune responses are dependent on host-origin or the MLVA genotype of C. burnetii strains. We assessed the immune responses in an in vivo mice infection model (Chapter 6) and by using an in vitro model of healthy human PBMCs (Chapter 7). From both the studies we saw a clear effect of host-origin grouping of $C$. burnetii strains rather than the MLVA genotype. Among different hostorigin strains, strains derived from cattle generated high pro-inflammatory cytokine-chemokines (IFN$\gamma$, TNF- $\alpha$, IL-1 $\beta$, IL-6, IL-12a, CCL2, CCL6, CXCL10), cell-mediated and antibody responses (IgG against phase I antigens) in mice compared to goat and human strains. Similarly, stimulation of human PBMCs with inactivated cattle strains also induced significantly more pro-inflammatory cytokines (TNF- $\alpha$, IL-1 $\beta$, IL-22) than goat, sheep and chronic human strains. Corresponding results from both studies showing protective immune responses to cattle strains could indicate that the mice and human immune system provoke an efficient defense against the pathogen, which could result in bacterial clearance and mild/ no disease symptoms. Whereas low immune responses to goat and human strains could result in less protection and prevent/delay elimination of these strains from the host leading to persistent infections and severe/chronic disease development. The differential immune responses generated by cattle, goat and human-derived strains reflect the observed differences in phenotypic expression of Q fever in natural hosts; where infected cattle rarely show clinical disease, contrasting the severe disease outcomes like abortions and chronic disease development in goats and humans respectively. Moreover, it might be possible that humans are less susceptible to cattle strains, as the human PBMCs elicits high pro-inflammatory response to cattle strains, which supports the molecular epidemiological observation (using MLVA) that cattle are rarely identified as the source of human Q 
fever outbreaks. Based on our findings, we suggest that bacteria originating from goat hosts have a greater potential to cause outbreaks in humans and needs to be efficiently monitored. Additionally, the cattle strains seem to be low-virulent strains as strong pro-inflammatory and protective immune response of the host upon infection indicate its reduced pathogenic potential.

In the general discussion (Chapter 8), the results of the thesis are presented and discussed in relation to the Dutch Q fever outbreak and the available international literature. Major outcomes of the thesis include the prominent genetic differences (several point mutations) and increased plasticity of the CbNL01 outbreak strains genomes, which might have resulted in its increased epidemic potential. Additionally, transcriptome analysis has contributed to an improved understanding of the pathogenesis of outbreak strains. The genome and transcriptome studies collectively showed up-regulation of most of the mutated genes of the outbreak strains under in vivo conditions, emphasizing the role of these genetic changes in evolutionary success of $C$. burnetii. The comparative analysis of several C. burnetii strains at the genome sequence level also showed a high similarity of strains within the same genotype. On the contrary, our results based on immune responses to various $C$. burnetii strains did not show any differences associated with the strain genotype, but only a strong association with its host-origin. This suggests that the virulence potential of $C$. burnetii strains is not only based on genetic differences, but also on other host-adaptation mechanisms such as transposition or differential gene regulation, which needs to be further investigated. Additionally, other non-genetic factors such as the risk to exposure (abortion storms in goat and sheep contrasting only mild disease in cattle), intensive goat farming and its proximity to the human populations could have facilitated the Dutch Q fever outbreak. One of the important outcomes highlighted in the discussion is the use of ultimate highresolution approaches like whole genome sequencing for molecular epidemiology and outbreak investigations of infectious diseases. This thesis also introduced and validated several basic and molecular tools to efficiently study virulence characteristics of intracellular bacterial pathogens. In addition, the present work provides a basis for future studies towards the development/design of vaccines and high-resolution diagnostic tools for $\mathrm{Q}$ fever, which could offer great potential in the control of $\mathrm{Q}$ fever outbreaks as well as limiting its transmission to humans. 

Samenvatting 


\section{Samenvatting}

De grootste Q koorts uitbraak gerapporteerd in Nederland heeft geleid tot ernstige ziekte bij de mens en tot massale ruiming van besmette geiten. Om dergelijke ziekte uitbraken in de toekomst te kunnen voorkomen, is het nodig om meer inzicht te verkrijgen in de ziekte veroorzakende bacterie. Dit inzicht zou kunnen leiden tot de ontwikkeling van vaccins gericht tegen Q koorts infecties. Het in dit proefschrift beschreven onderzoek richt zich op de karakterisering van C. burnetii stammen die bij de uitbraak hebben geleid tot een infectie in geiten, runderen, schapen en mensen. Hiervoor hebben we "pathogeen-omics" benaderingen gebruikt. Onze studies werden gestart om een beter inzicht te krijgen in de bacteriële pathogenese en om pathogeen gerelateerde factoren, die een rol hebben gespeeld bij de uitbraak, te kunnen identificeren. We onderzochten ook de immuun responsen die opgewekt werden tegen de verschillende $C$. burnetii stammen afkomstig van verschillende gastheren, in verschillende modelsystemen. Deze experimenten werden uitgevoerd om een immunologische verklaring te kunnen geven voor de waarneming dat $C$. burnetii stammen afkomstig van geiten hoog virulent zijn en leiden tot ziekte uitbraken, in tegenstelling tot stammen afkomstig van runderen. De resultaten beschreven in dit proefschrift hebben geleid tot meer kennis van de pathogeen geassocieerde moleculaire en immunologische factoren, die betrokken zijn bij gastheer-pathogeen interacties.

Om de verschillende studies die beschreven zijn in dit proefschrift beter te kunnen begrijpen, zorgt hoofdstuk 1 voor de benodigde achtergrond informatie over Q koorts en over de ziekte verwekker C. burnetii. Hieronder valt de algemene beschrijving van de Q koorts uitbraak in Nederland, de verschillende methodes die gebruikt werden om het onderzoek naar de uitbraken uit te voeren en de controlemaatregelen die genomen zijn om de ziekte bij de mens te beperken. Bovendien geeft dit hoofdstuk inzicht in de ontwikkelingen van het Q koorts onderzoek gedurende de afgelopen jaren en is er aandacht voor nog ontbrekende kennis rondom intracellulaire bacteriën. Dit alles is van belang om een meer kennis te verkrijgen over de ziekteverwekker, kan helpen bij het voorkomen van toekomstige Q koorts uitbraken en kan bijdragen aan de ontwikkeling van verbeterde diagnostiek of van effectieve vaccins.

De kennis over het metabole vermogen van C. burnetii is in 2003 sterk verbeterd door de publicatie van de eerste complete genoom sequentie. Dit heeft geleid tot de ontwikkeling van een axenisch medium waardoor vermeerdering van C. burnetii buiten eukaryote gastheercellen mogelijk werd. In hoofdstuk 2 beschrijven we onderzoek waarin we hebben gekeken of het virulente fenotype van C. burnetii-stammen stabiel wordt gehandhaafd na groei van de bacteriën in het cel vrije systeem vergeleken met groei van de bacteriën in gastheercellen (de gouden standaard). Deze experimenten werden uitgevoerd om te bevestigen dat groei in een cel vrij systeem niet leidt tot verlies van virulentie kenmerken. Ook hebben we de levensvatbaarheid van C. burnetii na een propidium monoazide (PMA) behandeling getest in een real-time PCR. Tevens werd de expressie van LPS 
coderende genen van de bacteriën in beide kweeksystemen bepaald. Expressie van LPS wordt beschouwd als een belangrijke parameter voor het vermogen van de bacterie om gastheercellen te kunnen infecteren. Als laatste hebben we een muis infectie model gebruikt om de virulentie van $C$. burnetii stammen gekweekt m.b.v. de beide kweeksystemen te beoordelen door de mate van splenomegalie en bacteriële verontreiniging in de milt te meten (geaccepteerde virulentie geassocieerde bepalingen). Onze studies toonden aan dat het virulente fenotype van de stammen in beide kweeksystemen werd gehandhaafd. Daarnaast werd in een C. burnetii stam, die zeer frequent werd gepasseerd in het cel vrije medium, een LPS modificatie van fase I naar II aangetoond. Een degelijke modificatie is kenmerkend voor een herhaaldelijke passage van C. burnetii stammen in andere in vitro modellen (zoals b.v. in bevruchte eieren en celculturen).

Omdat $C$. burnetii een obligaat intracellulaire pathogeen is, zal de bacterie zich snel metabool moeten kunnen aanpassen aan de extra- en intracellulaire omgeving van de gastheer cel. Om de differentiële genregulatie van de bacterie onder de verschillende groei omstandigheden te bestuderen, beschrijven we in hoofdstuk 3 het volledige expressie profiel van alle C. burnetii genen na in vivo infectie van muizen (milt) en na groei in verschillende in vitro modellen (cel en cel-vrij culturen). Tussen de bacteriën, gegroeid onder de verschillende condities, werden grote verschillen waargenomen in de expressieprofielen van metabole genen, maar ook van enkele met virulentie geassocieerde genen. Onder in vivo omstandigheden waren de meest opvallende waarnemingen de verhoogde expressie van genen coderend voor anionische cardiolipines en ion/proton wisselaars, die de bacterie gebruikt om de $\mathrm{pH}$ homeostase in stand te houden en te overleven binnen de sterk zure vacuolen van de gastheercellen; genen betrokken bij transport van organische moleculen, die duiden op de bacteriële afhankelijkheid van gastheer producten; genen die leiden tot een verhoogd anaeroob energiemetabolisme en verhoogde expressie van ROS enzymen en DNA herstelmechanismen die het de bacterie mogelijk maken om de oxidatieve stress in de gastheercellen te bestrijden. De in vivo omgeving leidt ook tot geïnduceerde expressie van een aantal bekende virulentiegenen van C. burnetii, waaronder genen die betrokken zijn bij de synthese van LPS en bij kolonisatie, maar ook van factoren geassocieerd met gastheercel modulaties (remming van apoptose of het nabootsen van gastheereiwitten). Dit betekent dat deze genproducten waarschijnlijk bijdragen aan de aanpassing van de bacterie aan de gastheer en de overleving van de bacterie onder in vivo omstandigheden. Deze studie geeft daarmee meer inzicht in de strategieën die de bacterie inzet om zich snel aan te kunnen passen aan de gastheer-specifieke omgeving en kan de basis vormen voor de identificatie van virulentiefactoren die van belang zijn voor het ontwerpen van vaccins.

In de hoofdstukken 4 en 5 beschrijven we de volledig gereconstrueerde genoomsequenties van C. burnetii-stammen verzameld van geiten, schapen en runderen tijdens de Nederlandse Q koorts uitbraak periode. Extra stammen verkregen uit Nederlandse chronische Q koorts patiënten werden ook 
meegenomen. In deze studies gebruikten we vergelijkende bioinformatica benaderingen en bevestigden dat de genoomsequenties van zowel geit en humane oorsprong beide behoorden tot het CbNL01 genotype, zoals vastgesteld in eerdere epidemiologische en genotypische studies. Die studies wezen er op dat geiten de bron waren voor de menselijke infecties tijdens de Nederlandse Q koorts uitbraak. Verder wezen fylogenetische analyses van de gesequencete stammen, samen met alle al gepubliceerde genoomsequenties van $C$. burnetii, op een genotype-specifieke clustering van de genomen. Dit duidt erop dat stammen die behoren tot één genotypische MLVA groep vergelijkbare genoomsequenties hebben. Hiermee toonden onze studies aan dat de meer routinematig toepasbare methodes, die nu frequent worden gebruikt om stammen te genotyperen (MLVA en MST), de genoom diversiteit goed weergeven. Hoewel "Whole Genome Sequencing" in de toekomst de voorkeur zal hebben, hebben MLVA en MST zeer waardevolle inzichten gegeven in de moleculaire epidemiologie van C. burnetii tijdens de Nederlandse Q koorts uitbraak. De gedetailleerde genetische verschillen tussen de CbNL01 uitbraak stammen, de referentie stam Nine Mile (NM) en stammen behorend tot het CbNL12 genotype werden ook in kaart gebracht. De genoom sequenties van deze stammen verschilden aanzienlijk. Er werden vooral veel puntmutaties gevonden en in de uitbraakstam was er een significante toename van het aantal transposase genen. Coderende puntmutaties werden gevonden in verschillende membraaneiwitten, DNA herstel genen, genen betrokken bij translatie, transport eiwitten, een Type IV secretie systeem en in potentiële virulentiefactoren. Het voorkomen van mutaties in genen die coderen voor membraaneiwitten met orthologen in NM en in CbNL12 stammen duidt er op dat deze genen onder selectiedruk antigene variatie hebben ondergaan. Daarnaast zijn er ook mutaties gevonden in genen die mogelijk betrokken zijn bij het veranderen van eiwitsynthese, bij eiwitexport en die leiden tot een toename van virulentie. Het toegenomen aantal transposons in de uitbraak stam vergeleken met andere stammen duidt op een toename in genoom plasticiteit. Een dergelijke plasticiteit kan van invloed zijn op de evolutie van het genoom van C. burnetii en is bekend te kunnen bijdragen aan een snelle aanpassing van de bacteriën aan een wisselende omgeving (bijvoorbeeld van extra- naar intracellulair). Al deze wijzigingen in C. burnetii genen en de uitgebreide transposon gemedieerde genoom re-arrangements kunnen hebben bijgedragen aan het toegenomen epidemische vermogen van de CbNL01 stammen.

In de hoofdstukken 6 en 7 hebben we de in hoofdstuk 5 beschreven stammen gebruikt om te identificeren of verschillen in de gastheer immuunreacties afhankelijk zijn van de gastheer waaruit de bacterie is geïsoleerd of van het MLVA genotype waartoe de stam behoort. We hebben de immuunrespons bestudeerd in een in vivo muis infectie model (hoofdstuk 6) en met behulp van een in vitro model van gezonde menselijke PBMCs (hoofdstuk 7). In beide studies zagen we dat immuun responsen opgewekt tegen $C$. burnetii stammen afkomstig van eenzelfde gastheer vergelijkbaar waren, terwijl de reactie tegen stammen met eenzelfde MLVA genotype dat niet waren. Ook genereerden de 
stammen afkomstig van runderen hogere pro-inflammatoire cytokine-chemokinen (IFN- $\gamma$, TNF- $\alpha$, IL$1 \beta$, IL-6, IL-12a, CCL2, CCL6, CXCL10), cel gemedieerde en antilichaam- responsen (IgG tegen fase I antigenen) in muizen dan stammen afkomstig van geiten en mensen. Bovendien leidde stimulatie van menselijke PBMCs met geïnactiveerde runder stammen tot aanzienlijk hogere pro-inflammatoire cytokine (TNF- $\alpha$, IL-1 $\beta$, IL-22) responsen dan stammen afkomstig van geiten, schapen en chronisch zieke mensen. Deze resultaten zouden er op kunnen wijzen dat muizen en mensen in staat zijn om een beschermende immuunrespons op te wekken tegen runder stammen waardoor er een efficiënte bescherming tegen de bacterie ontstaat. Dit kan resulteren in een efficiëntere verwijdering van de bacterie waardoor er mildere / geen ziektesymptomen ontstaan. Aan de andere kant zal de lage immuunrespons opgewekt tegen geiten en humane stammen leiden tot een laag niveau van bescherming waardoor de eliminatie van deze stammen wordt vertraagd en er ernstige infecties of chronische ziektes kunnen ontstaan. De differentiële immuunresponsen gegenereerd door stammen afkomstig van runderen, geiten en mensen weerspiegelen het verschil in het epidemiologisch succes van $C$. burnetii stammen waargenomen in de natuurlijke gastheren. Terwijl besmette runderen zelden klinische ziekte vertonen, ontwikkelen geiten en mensen ernstige ziekte, waaronder abortus en chronische infecties. Daarnaast zou het ook mogelijk kunnen zijn dat mensen minder gevoelig zijn voor runder stammen dan voor geiten stammen; omdat in humane PBMCs hoge pro-inflammatoire responsen worden opgewekt tegen runder stammen. Deze hypothese wordt ondersteund door moleculaire epidemiologische gegevens (MLVA) die laten zien dat runderen zelden worden geïdentificeerd als de bron van menselijke Q koorts uitbraken. Op basis van onze bevindingen lijkt het aannemelijk dat bacteriën afkomstig van geiten een hoog risico vormen voor het veroorzaken van uitbraken bij de mens. Deze geiten stammen moeten daarom efficiënt worden gemonitord. Runder stammen lijken daarentegen, op basis van de sterk pro-inflammatoire en beschermende immuunrespons die in de gastheer wordt opgewekt, laag-virulent.

In de algemene discussie (hoofdstuk 8) worden de resultaten van het proefschrift gepresenteerd en besproken in relatie tot de Nederlandse Q koorts uitbraak en de beschikbare internationale literatuur. De belangrijkste resultaten van het proefschrift zijn onder meer de opvallende genetische verschillen (meerdere puntmutaties) en verhoogde plasticiteit van de genomen van de Nederlandse CbNL01 uitbraak stammen. Deze verschillen kunnen hebben geleid tot het toegenomen epidemische vermogen van deze stammen. De transcriptoom analyse heeft bijgedragen aan een beter begrip van de pathogenese van de uitbraak stammen. De genoom en transcriptoom studies hebben beide laten zien dat de meeste van de gemuteerde genen van de uitbraak stammen onder in vivo omstandigheden sterker worden aangeschakeld. Dit onderstreept de rol van deze genetische veranderingen bij het evolutionaire succes van $C$. burnetii. De vergelijkende analyse van de genoomsequenties van een aantal C. burnetii-stammen liet een hoge mate van gelijkenis zien tussen 
stammen behorend tot eenzelfde genotype. C. burnetii stammen behorend tot hetzelfde genotype vertoonden op basis van de immuunresponsen echter geen overeenkomsten. Er was echter wel een sterke associatie tussen deze stammen op basis van gastheer-oorsprong. Dit suggereert dat de virulentie van C. burnetii-stammen niet alleen gebaseerd is op de genetische verschillen, maar ook op gastheer-adaptatie mechanismen zoals het optreden van transposon gemedieerde veranderingen of differentiële genregulatie. Dergelijke mechanismen zullen in de toekomst nog verder moeten worden onderzocht en zullen een belangrijke bijdrage kunnen leveren aan het bestuderen van intracellulaire bacteriële infecties. Bovendien kunnen andere niet-genetische factoren zoals het risico op blootstelling (contrast tussen de abortus storm in geiten en schapen en de milde ziekte verschijnselen bij runderen), intensieve geiten houderij en de nabijheid van de menselijke bevolking een rol hebben gespeeld bij het ontstaan en de efficiënte verspreiding van Q koorts in Nederland. Dit proefschrift onderschrijft het belang van het gebruik van hoge resolutie benaderingen zoals, whole genome sequencing technieken voor moleculair epidemiologische studies en onderzoek naar infectieziekte uitbraken. In dit proefschrift worden een aantal C. burnetii-specifieke moleculaire tools geïntroduceerd en gevalideerd, waarmee ook virulentie kenmerken van intracellulaire bacteriële pathogenen in het algemeen efficiënter kunnen worden bestudeerd. Bovendien, biedt het huidige werk een basis voor nieuwe studies naar de ontwikkeling van vaccins en diagnostische tools voor Q koorts. Dergelijke tools die mogelijk snel een virulenter type zouden kunnen aantonen zouden van grote meerwaarde kunnen zijn bij de bestrijding van Q koorts uitbraken evenals bij het voorkomen van de overdracht van C. burnetii van dier naar mens. 



\section{Curriculum vitae}




\begin{abstract}
About the Author
Runa Kuley was born on the $7^{\text {th }}$ of July 1988 in Kolkata, West Bengal, a state in the East India. She spent her youth and finished schooling at Hyderabad, a city located in the southern India. She received Bachelors in Biotechnology from Osmania University, Hyderabad in 2009 and after that joined the University of Hyderabad and obtained Masters Degree in Animal Biotechnology in 2011. Her master thesis in the Department of Neurobiology at University of Hyderabad was focused on investigating the effect of melatonin on age-induced changes in relation to oxidative stress in peripheral clocks in rats. During Masters she received "Achiever's Award" and was a "Gold medalist" for academic excellence. In September 2011 she started her PhD at Wageningen University \& Research, The Netherlands. Her laboratory work was conducted at the Department of Infection Biology, Wageningen Bioveterinary Research, Lelystad, The Netherlands. Her research was focused on "Characterization of outbreak strains of Coxiella burnetii". The results of her $\mathrm{PhD}$ research are presented in this thesis.
\end{abstract}




\section{List of Publications}

Kuley R, Smith HE, Frangoulidis D, Smits MA, Jan Roest HI, Bossers A. Cell-Free Propagation of Coxiella burnetii Does Not Affect Its Relative Virulence. PLoS ONE. 2015; 10:e48949

Kuley R, Bossers-deVries R, Smith HE, Smits MA, Roest HIJ, Bossers A. Major differential gene regulation in Coxiella burnetii between in vivo and in vitro cultivation models. BMC Genomics. $2015 ; 16: 953$.

Kuley R, Smith HE, Janse I, Harders FL, Baas F, Schijlen E, Nabuurs-Franssen MH, Smits MA, Roest HI, Bossers A. First Complete Genome Sequence of the Dutch Veterinary Coxiella burnetii Strain NL3262, Originating from the Largest Global Q Fever Outbreak, and Draft Genome Sequence of Its Epidemiologically Linked Chronic Human Isolate NLhu3345937. Genome Announcements. 2016 Apr 21;4(2). pii: e00245-16. doi: 10.1128/genomeA.00245-16.

Ammerdorffer A, Kuley R, Jan Roest HI. Physiopathology of Coxiella burnetii infection and host immunologic response. Book Title: The Principles and Practice of Q Fever: The One Health Paradigm.

Ammerdorffer A, Kuley R, Dinkla A, Sprong T, Jan Roest HI, Rebel JM. Coxiella burnetii isolates originating from infected cattle induce a more pronounced pro-inflammatory cytokine response compared to isolates from infected goats and sheep. Pathogens and Disease, 2017, doi: doi.org/10.1093/femspd/ftx040. 


\section{Submitted Manuscripts}

Kuley R, Smith HE, Smits M, Jan Roest HI, Bossers A. Coxiella burnetii strain specific immune responses are more depending on host origin than on MLVA genotype classification. Infection and Immunity, Under review

Kuley R, Smith HE, Smits M, Jan Roest HI, Bossers A. Plasticity and polymorphisms in critical genes correlate with increased virulence of Dutch outbreak-related Coxiella burnetii strains. Frontiers in Microbiology, Under review 

Acknowledgements 


\section{Acknowledgements}

The remarkable $\mathrm{PhD}$ journey has come to an end and throughout this journey I have benefited from the help and support from my family, friends and colleagues. I would like to take this opportunity to thank everyone from the bottom of my heart without whom preparation of this thesis would not have been possible.

First of all, I would like to express my appreciation and gratitude towards my co-promoter Dr. Alex Bossers. Words cannot express all that I wish to say to you. I am grateful for the care and support you have shown towards me right from the first day I reached Lelystad till my last day in the Netherlands. I am very thankful for your constant supervision, guidance, enthusiasm and encouragement throughout my stay at Wageningen Bioveterinary Research. I have very much enjoyed our continuous discussions on various topics, especially on bioinformatics that helped me a lot in my research. Thank you for always having an open mind about new ideas which has helped me enormously to work independently. Finally, thanks for expressing your appreciation for even the smallest of my achievements, it always motivated me a lot. You were really inspiring and I hope to meet more of such persons later in my career.

I would like to thank my promoter Prof. M.A. Smits for taking care that everything went smoothly during my $\mathrm{PhD}$. Mari I am really grateful to you for your critical comments on my research work and the time you have invested in looking closely into the chapters which has really improved my thesis. I extend my gratefulness to my supervisors Dr. H. Smith and Dr. H.I.J. Roest for their continuous support and valuable suggestions on different research projects. A special thanks to Hilde for writing the Dutch summary of my thesis. I would like to thank Prof. J.M. Wells for his contributions to this thesis.

I am thankful to Annemieke for the time she spent in training me to work in Biosafety Level-3 facilities. You were also very helpful in my earlier days to setup my lab work. Despite your busy schedule, you always found some time for me whenever I needed you, I could not have had a better help!

I take this opportunity to thank my paranimfs Apostolos and Eric for their help in preparation for my defense. Apostolos, I have enjoyed each and every bit of time we spent together whether it was at Jan's house or at the institute. Also I would like to thank you for your words of encouragement and support whenever I needed it. Eric, thank you for helping me with the data analysis. I have learnt a lot 
from you and enjoyed numerous scientific and non-scientific discussions that we had. I am really grateful to you for helping me with the technical difficulties that I faced during my research.

I earnestly express my gratitude to Ruth for helping me with the microarray experiments and extending her expertise to my research. I would like to thank Frank for his help in sequencing projects. Special thanks to my colleagues Conny, Jan, Nadia, Astrid, Michiel, Paul, Albert, Rene, Betty, Herma, Lisette, Lucien, Rene and Jacob for helping me in various experiments. Jan, Toon, Rene and Conny, it was a pleasure to share office space with you all and thank you for decorating the room on my birthdays.

I would like to express my gratitude to all my co-authors, who contributed to the work described in this thesis. Anne, I enjoyed working with you during the times you were at Lelystad. It was always fun catching up with you and discussing about Q fever. I would also like to thank Ad for his expert guidance in immunology-related work presented in this thesis.

My stay in Netherlands could not be easier if it was not for Karel and Mike. Thanks to both of you for offering me to ride with you to Groningen. I would also like to thank my colleagues, past and present at Wageningen Bioveterinary Research for providing a pleasant ambience at the workplace.

Dear animal caretakers, thank you for taking care of the animals and helping me with the experiments.

My sincere thanks to Ria, Randi and Loes for their help in handling official procedures during my stay in The Netherlands.

Outside the workplace, I would like to thank my friends Ritika, Vijay, Harinakshi, Jide and Fang for the memorable times we spent together. Fortunately, I had good times with two of my socially active colleagues. Apostolos, I enjoyed all the trips we had taken together and I really appreciate that you travelled all the way to India to attend my wedding. It was wonderful to share my happiness with you. Michiel, thanks for all the time we spent together and sharing stories of your adventurous holidays.

One of the strengths during my stay at Netherlands was Jan Maat. I enjoyed living in your house. You helped me whenever I needed it regardless of what time of the day it was. I really appreciate that you actively hosted my family when they were visiting me in The Netherlands and showed them around when I was at work. You are a nice host and a much nicer person, I feel grateful knowing you. 
It was great having you in my wedding. It was also nice to know your family and I would like to thank them for the love they showed towards me. Your constant support made my journey much easier and made it feel like home away from home.

I am extremely thankful to my husband Sanjeev for his love, encouragement, advice and support the whole time, even at the most difficult moments that made my journey easier. In 2011, I was not sure if it was the right decision for me to pursue a $\mathrm{PhD}$ in The Netherlands. Today I have no doubt that it was the best decision I have ever made. In true sense we embarked on an adventure together at The Netherlands. I could not have made it without you. Thank you, from all my heart, I feel very lucky to have you in my life and it is a great fortune to have you love me.

Finally, I would like to thank my family members for their continuous support throughout my life. I am very grateful to my father, mother, brothers and sister-in-law for their love, faith, affection and always remembering me in their good thought. I also thank my in-laws for their support during the thesis completion period. I specially would like to thank my father who always supported me in my decisions and encouraged me to follow my dreams. 



\section{Training and supervision plan}




\section{Overview of completed training activities}

\section{Conferences and meeting}

International Q Fever Symposium, Amsterdam, The Netherlands, 2012 (Oral Presentation)

National Q fever meeting, RIVM, The Netherlands, 2012

National Q fever meeting, RIVM, The Netherlands, 2013 (Oral Presentation)

Med-Vet-Net Association International conference, Lyngby, Denmark, 2013 (Poster Presentation)

Spring Meeting KNVM/NVMM, Arnhem, The Netherlands, 2013 (Poster Presentation)

WIAS Science day, Wageningen, The Netherlands, 2013 (Poster Presentation)

WIAS Science day, Wageningen, The Netherlands, 2014 (Poster Presentation)

Agilent plant and animal symposium, Amsterdam, The Netherlands, 2014 (Oral Presentation)

SFAM/MVNA Summer conference, Brighton, UK, 2014 (Oral and Poster Presentation)

Spring Meeting KNVM/NVMM, Arnhem, The Netherlands, 2014 (Poster Presentation)

$27^{\text {th }}$ Meeting of the American Society for Rickettsiology, California, USA, 2015 (Oral Presentation)

WIAS Science day, Wageningen, The Netherlands, 2015 (Oral Presentation)

CSHL Meeting: Microbial Pathogenesis \& Host Response, New York, USA (Poster Presentation)

\section{Courses}

In-Depth Studies

EBI Course: Sequence searching and alignment, 2012

Course on Laboratory Animal Science, 2012

EPI - PhD Course 'Bioinformatics - a User's Approach, 2013

Spring School 'Host-Microbe Interactomics, 2014

Next-Generation Sequencing, 2014

Statistics for the Life Sciences, 2014

\section{General}

Information Literacy including EndNote, 2011

WIAS Introduction Course, 2012

WIAS Course Ethics and Philosophy in Life Sciences, 2012

High Impact writing for academic publications, 2013

Writing for academic publications, 2014

\section{Research Skills Training}

Preparation of PhD proposal, 2011 


\section{Colophon}

This research in this thesis was conducted at the Wageningen Bioveterinary Research, Department of Infection Biology, Lelystad, The Netherlands. The research was financially supported by Dutch Ministry of Economic Affairs WOT-01-002-005.06, WOT-01-002-005.05, KB-12-005.01-019, and KB-12-005.01-005.

The cover of this thesis was designed by Runa Kuley and Sanjeev Kumar.

This thesis was printed by Digiforce || Proefschriftmaken.nl, Vianen, NL. 
